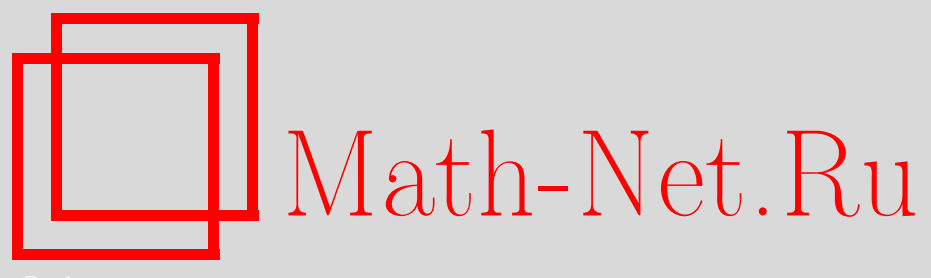

Л. Эрдёш, Универсальность случайных матриц Вигнера: обзор последних результатов, УМН, 2011, том 66, выпуск 3, 67-198

DOI: https://doi.org/10.4213/rm9426

Использование Общероссийского математического портала Math-Net.Ru подразумевает, что вы прочитали и согласны с пользовательским соглашением http://www . mathnet.ru/rus/agreement

Параметры загрузки:

IP: 54.147 .182 .235

26 апреля 2023 г., 15:49:47

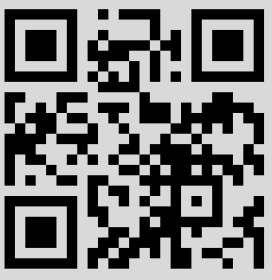




\section{Универсальность случайных матриц Вигнера: обзор последних результатов}

\section{Л. Эрдёш}

Мы изучаем свойства универсальности спектральных статистик больших случайных матриц. Рассматриваются симметрические, эрмитовы или кватернионные самодвойственные случайные матрицы с независимыми одинаково распределенными элементами (матрицы Вигнера), причем распределение вероятностей каждого матричного элемента задается мерой $\nu$ с нулевым математическим ожиданием и субэкспоненциальным убыванием. Наш основной результат заключается в том, что корреляционные функции локальных статистик собственных значений внутри спектра совпадают с корреляционными функциями гауссова ортогонального ансамбля (ГОА), гауссова унитарного ансамбля (ГУА) и гауссова симплектического ансамбля (ГСА) соответственно, в пределе при $N \rightarrow \infty$. Наш подход основан на изучении броуновского движения Дайсона при помощи соответствующей новой динамики - локального релаксационного потока.

Главным средством доказательства является утверждение о том, что плотность собственных значений сходится к полукруговому закону Вигнеpa, и это имеет место даже для наименьшего возможного порядка величин; более того, мы показываем, что собственные векторы полностью делокализуются. Эти результаты справедливы даже при отсутствии условия одинаковой распределенности матричных элементов, требуется лишь их независимость. Мы даем сильные оценки на матричные элементы функции Грина, при выполнении которых локальные статистики двух ансамблей совпадают внутри спектра при совпадении первых четырех моментов матричных элементов. Универсальность на границах спектра требует совпадения лишь двух моментов. Мы также доказываем оценки типа Вегнера и тот факт, что собственные значения отталкивают друг друга при произвольно малых порядках величин.

Библиография: 108 названий.

Ключевые слова: случайные матрицы Вигнера, броуновское движение Дайсона, полукруговой закон, синус-ядро.

\section{СОДЕРЖАНИЕ}

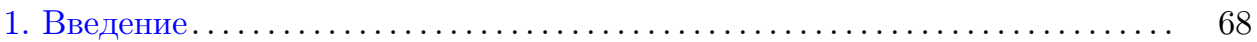

1.1. Обзор основных результатов: руководство для читателя ....... 70

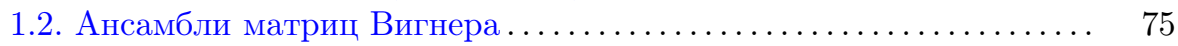

Работа частично поддержана грантом SFB-TR 12 Научного Совета Германии. 
1.3. Мотивации: от операторов Шрёдингера к $\zeta$-функциям ........ 78

1.4. Плотность собственных значений и делокализация........... 82

1.5. Локальные статистики собственных значений: предыдущие ре-

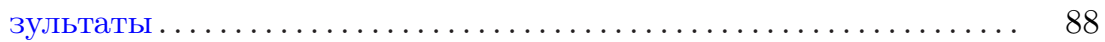

1.6. Локальные статистики собственных значений: новые результаты 99

1.7. Отталкивание уровней и оценка Вегнера для очень малых масштабов ...................................... 119

2. Локальный полукруговой закон и делокализация................. 123

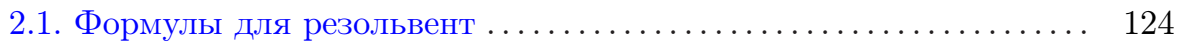

2.2. Полукруговой закон при помощи резольвент: набросок грубого

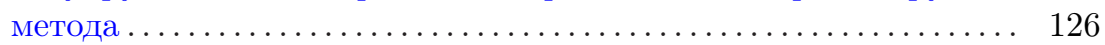

2.3. Полукруговой закон из резольвент: улучшенный метод . . . . . . . 131

2.4. Сильный локальный полукруговой закон ................ 148

2.5. Делокализация собственных векторов ................... 151

3. Универсальность для гауссовых конволюций .................... 154

3.1. Сильная локальная эргодичность броуновского движения Дай-

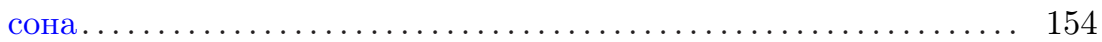

3.2. Локальный релаксационный поток.................. 157

3.3. От распределения промежутков к корреляционным функциям: набросок доказательства теоремы $3.1 \ldots \ldots \ldots \ldots \ldots \ldots \ldots \ldots$

4. Теоремы сравнения для функций Грина ..................... 170

4.1. Доказательство теоремы 4.1 сравнения для функций Грина . . . 173

4.2. Доказательство теоремы 4.2 о сравнении корреляционных

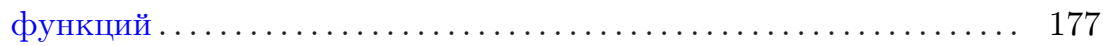

4.3. Набросок доказательства теоремы $1.7 \ldots \ldots \ldots \ldots \ldots \ldots \ldots \ldots . \ldots . \ldots . \ldots 18$

5. Универсальность для матриц Вигнера: сведение компонент доказатель-

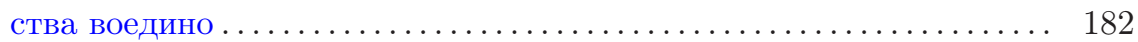

5.1. Шаг 1: универсальность для гауссовых конволюций........... 185

5.2 . Шаг 2: лемма о совпадении ..................... 186

Приложение А. Оценки больших уклонений: доказательство леммы 2.12 188

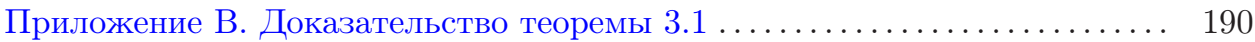

Список литературы ...................................... 193

\section{1. Введение}

Данный обзор основан на записях лекций, подготовленных автором для участников Аризонской школы по анализу и приложениям (Тусон, США, 2010 г.). Принятый здесь стиль изложения ближе к неформальному лекционному стилю, чем к стилю формальной исследовательской статьи. За подробностями, а иногда даже и за точными формулировками утверждений мы отсылаем читателя к исходным работам.

В первом разделе мы приводим обзор свойств универсальности случайных матриц, включая предыдущие результаты, историю вопроса и мотивации. Мы вводим некоторые основные понятия, такие как матрицы Вигнера, полукруговой закон Вигнера, преобразование Стилтьеса, метод моментов, синус-ядро, распределение промежутков, отталкивание уровней, универсальность внутри 
спектра и на его границах, инвариантные ансамбли, теорема о сравнении функций Грина, теорема о четырех моментах, локальный релаксационный поток и обратный тепловой поток. Часть этих понятий не будет использована в наших основных результатах, но мы включаем их для того, чтобы читатель мог в них ориентироваться. Подбор материала в первом разделе отражает предпочтения автора и не может считаться исчерпывающим. Основной целью здесь является подготовка основ для последующих разделов, где мы излагаем наши последние результаты об универсальности случайных матриц.

Имеется несколько очень активно исследуемых направлений, связанных со случайными матрицами, которые совершенно не отражены в нашем обзоре; например, суперсимметрические методы или взаимосвязи со свободной вероятностью. Другие весьма обширные разделы, например, универсальность на границах и метод моментов или взаимосвязи с ортогональными многочленами, упоминаются лишь поверхностно. Мы отсылаем читателя к более подробным обзорам по случайным матрицам, например, к классической книге Меты [1], обзору Дефта [2] по подходу Римана-Гильберта, недавним книгам Андерсона, Гионне и Цайтуни [3] и Форрестера [4]. Прекрасный краткий обзор последних достижений дан Гионне [5].

Начиная с раздела 2, мы излагаем наши последние результаты, которые дают кратчайший и на данный момент наиболее мощный метод доказательства универсальности внутри спектра для $(N \times N)$-матриц Вигнера. Один из наших основных результатов сформулирован в теореме 5.2. Эти результаты были получены в сотрудничестве с Дж. Рамиресом, С. Пеше, Б. Шлейном, Х.-Т. Яу и Дж. Ином; точные ссылки даны в списке литературы. В этой части обзора мы стремились изложить математические рассуждения максимально полно и точно, тем не менее иногда мы будем отсылать читателя к исходным работам за некоторыми подробностями. Рассуждение разбивается на три отдельных шага:

1) локальный полукруговой закон (раздел 2);

2) доказательство универсальности гауссовых конволюций на основе локального релаксационного потока (раздел 3);

3) теорема сравнения для функций Грина (раздел 4).

Наконец, в разделе 5 мы объединяем эти составляющие в одно доказательство. Основной результат об универсальности локальных статистик матриц Вигнера сформулирован в теореме 5.1. Ряд технических лемм собраны в приложениях; при первом чтении их можно опустить.

СогЛАшЕНиЕ. На протяжении всей статьи буквами $C$ и $c$ будут обозначаться положительные константы, значения которых будут меняться от формулы к формуле и которые будут независимыми от остальных параметров. Так как в конце концов мы всегда переходим к пределу при $N \rightarrow \infty$, все оценки будут выполняться для достаточно больших $N$. При неформальных пояснениях мы обычно не будем учитывать логарифмические множители; с этой целью мы вводим обозначения $\lesssim$ и для неравенств "с точностью до некоторого $\log N$-множителя". Более точно, $A \lesssim B$ означает $A \leqslant(\log N)^{C} B$ для некоторой неотрицательной константы $C$, а $A \ll B$ означает $A \leqslant(\log N)^{-C} B$ для некоторой положительной константы $C$. 
Автор благодарен Х.-Т. Яу за предложения по улучшению изложения этого обзора.

1.1. Обзор основных результатов: руководство для читателя. Мы будем рассматривать матрицы $H=\left(h_{i j}\right)_{i, j=1}^{N}$ размера $N \times N$, элементами которых являются действительные или комплексные случайные величины. В большинстве случаев мы предполагаем, что матрица $H$ эрмитова или симметрична, однако наш метод применим и к другим ансамблям матриц (наши результаты о матрицах с кватернионными элементами здесь обсуждаться не будут, см. [6]). Мы предполагаем (в дополнение к ограничению симметричности $h_{i j}=\bar{h}_{j i}$ ), что матричные элементы независимы, центрированы, т. е. $\mathrm{E} h_{i j}=0$, a их хвостовые вероятности убывают по равномерному субэкспоненциальному закону (см. (2.32) ниже). Мы не предполагаем, что матричные элементы одинаково распределены, а предполагаем лишь, что дисперсии $\sigma_{i j}^{2}:=\mathrm{E}\left|h_{i j}\right|^{2}$ удовлетворяют условию нормировки

$$
\sum_{j=1}^{N} \sigma_{i j}^{2}=1, \quad i=1, \ldots, N
$$

т. е. детерминированные $(N \times N)$-матрицы дисперсий $\Sigma=\left(\sigma_{i j}^{2}\right)$ являются симметрическими и дважды стохастическими. Эти условия гарантируют, что $-1 \leqslant$ $\Sigma \leqslant 1$. Мы будем всегда предполагать, что 1 является простым собственным значением матрицы $\Sigma$ и что найдется положительное число $\delta_{-}>0$ такое, что $-1+\delta_{-} \leqslant \Sigma$. Это условие выполнено практически для всех ансамблей случайных матриц. Иногда нам будет требоваться условие равномерности промежутков, т. е. существование положительного $\delta_{+}>0$ такого, что

$$
\operatorname{Spec} \Sigma \subset\left[-1+\delta_{-}, 1-\delta_{+}\right] \cup\{1\} \text {. }
$$

Например, для стандартной матрицы Вигнера мы имеем $\sigma_{i j}^{2}=N^{-1}$ и $\delta_{-}=$ $\delta_{+}=1$. Для случайных ленточных матриц (см. точное определение в (1.18)) с шириной “ленты” $W$, удовлетворяющей условию $1 \ll W \ll N$, промежуток $\delta_{+}$ стремится к нулю с увеличением размера матрицы.

Условие нормализации (1.1) влечет, что почти весь спектр матрицы $H$ лежит в интервале $[-2,2]$, а плотности собственных значений $\lambda_{1} \leqslant \cdots \leqslant \lambda_{N}$ задаются полукруговым законом Вигнера при $N \rightarrow \infty$. За исключением окрестностей границ \pm 2 , типичное расстояние между соседними собственными значениями имеет порядок $1 / N$. Нас интересуют статистики собственных значений в пределе при $N \rightarrow \infty$.

1.1.1. Обзор раздела 2: основные результаты, связанные с локальным полукруговым законом. В разделе 2 мы доказываем, что плотности собственных значений удовлетворяют полукруговому закону вплоть до наименъшего возможного масштаба, т. е. до порядка величин немного больше $1 / N$. Мы будем называть это свойство локалъным полукруговым законом. Локальный полукруговой закон определяется при помощи преобразования Стилтьеса эмпирической плотности собственных значений,

$$
m(z):=m_{N}(z)=\frac{1}{N} \sum_{j=1}^{N} \frac{1}{\lambda_{j}-z}, \quad z=E+i \eta, \quad E \in \mathbb{R}, \quad \eta>0
$$


и мы показываем, что $m_{N}(z)$ сходится к преобразованию Стилтьеса плотности полукруга

$$
m_{\mathrm{sc}}(z):=\int_{\mathbb{R}} \frac{\varrho_{\mathrm{sc}}(x) \mathrm{d} x}{x-z}, \quad \varrho_{\mathrm{sc}}(x):=\frac{1}{2 \pi} \sqrt{\left(4-x^{2}\right)_{+}},
$$

в пределе при $N \rightarrow \infty$. Мнимая часть $\eta=\operatorname{Im} z$ может зависеть от $N$, что соответствует локальному масштабу, для которого определяется плотность. Точность нашего приближения имеет порядок $(N \eta)^{-1}$. Нашим наилучшим результатом в этом направлении является теорема 2.1 из [7], которую мы будем называть силъным локалъным полукруговым законом:

$$
\left|m(z)-m_{\mathrm{sc}}(z)\right| \leqslant \frac{C(\log N)^{L}}{N \eta}
$$

для некоторого достаточно большого $L$ и с очень высокой вероятностью (см. п. 2.4). Этот результат имеет место даже для более общего класса матриц Вигнера, дисперсии которых сравнимы (точное определение см. в (1.17)); ключевым моментом здесь является тот факт, что в этом случае мы имеем $\delta_{+}>0$.

Для матриц Вигнера еще более общего вида (они будут называться универсальными матрицам Вигнера, см. определение 1.1 ниже) ключевой величиной, измеряющей точность оценки, является ширина матрицы, определяемая как

$$
M:=\frac{1}{\max _{i j} \sigma_{i j}^{2}} .
$$

Для типичных случайных ленточных матриц (точное определение см. в (1.18)) ширина $M$ сравнима с шириной ленты $W$. При $M \ll N$ точность наших оценок определяется в терминах $M$, а не $N$. Например, в [8] получена оценка

$$
\left|m(z)-m_{\mathrm{sc}}(z)\right| \leqslant \frac{C N^{\varepsilon}}{M \eta \kappa^{2}}, \quad \kappa:=|| E|-2|,
$$

для всех $\varepsilon>0$ с очень высокой вероятностью (см. теорему 2.5 ниже); кроме того, в [9; теорема 2.1] была доказана оценка

$$
\left|m(z)-m_{\mathrm{sc}}(z)\right| \leqslant \frac{C(\log N)^{L}}{\sqrt{M \eta} \kappa} .
$$

Заметим, что эти оценки ухудшаются вблизи границ спектра.

Хорошо известно, что задание преобразования Стилтьеса некоторой меры для комплексных параметров $z=E+i \eta, E \in \mathbb{R}$, эквивалентно заданию плотности вплоть до порядка величин примерно $O(\eta)$; тем самым мы получаем контроль над плотностью вплоть до порядка величин примерно $\eta \sim 1 / M$.

Преобразование Стилтьеса $m(z)$ можно также рассматривать как нормированный след резольвенты,

$$
m(z)=\frac{1}{N} G(z)=\frac{1}{N} \sum_{i=1}^{N} G_{i i}(z), \quad G(z):=\frac{1}{H-z} .
$$


Наряду с (1.2), мы можем доказать, что не только сама сумма, но и каждый диагональный элемент $G_{i i}(z)$ задается полукруговым законом, хотя и с несколько меньшей точностью:

$$
\max _{i}\left|G_{i i}(z)-m_{\mathrm{sc}}(z)\right| \lesssim \frac{C}{\sqrt{N \eta}}, \quad z=E+i \eta .
$$

Наконец, мы также показываем, что внедиагональные элементы резольвенты малы:

$$
\max _{i j}\left|G_{i j}(z)\right| \lesssim \frac{C}{\sqrt{N \eta}}
$$

с логарифмическими поправками [7] (см. теорему 2.19 в п. 2.4). В наших предыдущих работах [9], [8] константы $C$ в формулах (1.6) и (1.7) зависели от $\kappa$, т. е. оценки ухудшались вблизи границ спектра как обратная степень $\kappa$; показатель этой степени зависит от того, имеется ли положительная равномерная нижняя граница $\delta_{+}>0$. Для более общих матриц Вигнера, например, для ленточных матриц, мы получаем аналогичные оценки, в которых $N$ заменяется на $M$ в правой части формул (1.6) и (1.7), а $C$ зависит от $\kappa$. Точные формулировки даны в теореме 2.5.

Асимптотики преобразования Стилтьеса можно перевести в асимптотики функции подсчета (см., например, теорему 2.6) или в результат о расположении собственных значений (теорема 2.7). Более того, локальный полукруговой закон влечет, что собственные векторы полностью делокализуются (см. п. 2.5).

1.1.2. Обзор раздела 3: основные результаты об универсальности внутри спектра с гауссовой компонентой. Свойство универсальности внутри спектра выражает тот факт, что локальные статистики собственных значений, т. е. корреляционные функции собственных значений, отмасштабированные в $N$ раз, а также распределение промежутков между последовательными собственными значениями демонстрируют универсальное поведение, которое определяется исключительно классом симметрии ансамбля.

Универсальность внутри спектра была впервые доказана Дайсоном [10] и Метой [11] для гауссовых ансамблей Вигнера, т. е. в случае, когда матричные элементы $h_{i j}$ являются независимыми одинаково распределенными гауссовыми случайными величинами. Гауссов характер распределения делает явные вычисления более простыми, что необходимо для определения предельных корреляционных функций (например, знаменитое синус-ядро в эрмитовом случае). Ключевым фактом является то, что совместная функция распределения для собственных значений таких ансамблей выписывается явно и имеет структуру определителя Вандермонда, из которой вытекает локальная универсальность, см. (1.44).

Естественным образом возникает идея рассмотреть более общий класс матриц, характер распределения которых связан с гауссовым. Полезным здесь является понятие гауссово делимых ансамблей, для которых вероятностный закон каждого матричного элемента содержит гауссовы компоненты (гауссовы конволюции).

Один из подходов к изучению гауссовых конволюций заключается в том, чтобы путем дальнейших явных вычислений установить аналогичную структуру определителя Вандермонда. Опираясь на более раннюю работу Брезина 
и Хиками [12], Йоханссон [13] нашел формулу представления для корреляционных функций и смог доказать универсальность для гауссово делимых матриц. Ввиду алгебраических ограничений, этот метод работает лишь в эрмитовом случае.

В [13] был существен размер гауссовой компоненты; его порядок равнялся порядку размера негауссовой части. Используя наш локальный полукруговой закон и несколько модифицированный вариант явной формулы представления Йоханссона [13], нам удалось доказать в [14] (п. 1.6.1) универсальность для эрмитовых матриц Вигнера с пренебрежимо малыми гауссовыми компонентами, дисперсии которых имеют порядок $O\left(N^{-1+\varepsilon}\right)$, с улучшенной формулой.

Другой подход (кратко изложенный в п. 1.6.2 и подробно в разделе 3) заключается в том, чтобы вложить гауссово делимый ансамбль в стохастический поток матриц и затем использовать ключевое наблюдение Дайсона [15], что под действием этого потока собственные значения имеют специфическую стохастическую динамику с логарифмическим взаимодействием - знаменитое броуновское движение Дайсона. В конце концов эта динамика приходит в равновесие, которое является хорошо известной гауссовой моделью (ГУА, ГОА или ГСА). Основная идея заключается в том, что локальная релаксация происходит намного быстрее, т. е. локальные статистики собственных значений достигают своего равновесия в течение очень короткого времени порядка $t=N^{-\varepsilon}$ (с явно задаваемым $\varepsilon>0)$. В действительности Дайсоном было предсказано в [15], что порядок времени прихода в локальное равновесие равен $N^{-1}$. Это в конце концов было доказано нами в [7]. Наш основной результат заключается в том, что локальные корреляционные функции гауссово делимых матриц с малыми гауссовыми компонентами совпадают с корреляционными функциями чисто гауссовых ансамблей.

Этот результат можно сформулировать в общем виде и рассматривать как свойство сильной локальной эргодичности для броуновского движения Дайсона или даже для любой одномерной стохастической динамики частиц с логарифмическим взаимодействием. Данная общая формулировка впервые появилась в [6] и будет приведена в теореме 3.3 , хотя большинство ключевых идей возникло уже в работе [16]. Для того чтобы приложить данный общий принцип к случайным матрицам, необходима некоторая априорная информация о распределении собственных значений, которую мы получаем из локального полукругового закона. В частности, эта идея была использована в доказательстве универсальности для симметричных матриц Вигнера в [16]. Случай кватернионных самодвойственных и выборочных ковариационных матриц был рассмотрен в [6].

1.1.3. Обзор раздела 4: основные результаты об удалении гауссовых компонент. Для доказательства универсальности любого ансамбля Вигнера нам требуется сравнить его с гауссово делимым ансамблем, для которого универсальность уже была доказана. Такой принцип сравнения возможен, если гауссова компонента мала, и в этом случае действительно может быть применено рассуждение с возмущениями. В действительности оно является рассуждением о плотности, утверждающим, что гауссово делимые ансамбли достаточно "плотны" в пространстве всех ансамблей Вигнера. 
Первый результат в этом направлении использовал рассуждение с обращенным тепловым потоком [14], где нами было показано, что любое гладкое распределение может быть приближено гауссово делимым распределением с очень высокой точностью. Используя этот метод, а также свойство универсальности для эрмитовых матриц Вигнера с гауссовой компонентой дисперсии порядка $O\left(N^{-1+\varepsilon}\right)$, нам удалось доказать универсальность для любого ансамбля Вигнера при условии, что распределение матричных элементов является гладким.

Более грубый подход основан на теореме сравнения для функций Грина из [9], которая утверждает, что совместное распределение функций Грина двух матричных ансамблей совпадает при условии, что первые четыре момента вероятностного закона для матричных элементов совпадают или очень близки. Спектральный параметр $z$ может иметь очень малую мнимую часть $\operatorname{Im} z \sim$ $N^{-1-\varepsilon}$, т. е. эти функции Грина могут выявлять индивидуальные собственные значения. Точное утверждение дано в теореме 4.1. Ключевым элементом здесь является локальный полукруговой закон, включающий индивидуальные матричные элементы резольвенты (см. (1.6)-(1.7)).

Совместное применение результатов п. 1.1.2 об универсальности гауссово делимых матриц и теоремы сравнения для функций Грина позволяет установить универсальность для любого ансамбля Вигнера, используя простое рассуждение о совпадении [8]. Этот метод применим также для матриц со сравнимыми дисперсиями (1.17). Единственным условием для его применения является субэкспоненциальное убывание хвостов вероятностного закона для матричных элементов (2.32). В действительности это условие может быть ослаблено до достаточно быстрого полиномиального убывания, но для простоты мы не будем рассматривать результаты в этом направлении.

Условие четырех моментов было впервые отмечено Тао и Ву [17] в теореме о четырех моментах для собственных значений (теорема 1.5). Ключевым техническим элементом их доказательства был по-прежнему локальный полукруговой закон и его следствие о делокализации собственных векторов. Они использовали этот результат для доказательства универсальности для эрмитовых матриц Вигнера без условия гладкости, но с некоторым условием на моменты и носитель, которое, в частности, исключало распределение Бернулли. Универсальность для эрмитовых матриц, включая случай распределения Бернулли, была впервые доказана в [18] на основе совместного применения результатов из [14] и [17].

Наконец, в разделе 5 мы формулируем главный результат (теорему 5.1) об универсальности и описываем то, каким образом его доказательство может быть получено из результатов предыдущих разделов. В настоящий момент совместное применение метода локальной релаксации потока и теоремы сравнения для функций Грина дает наиболее общий подход к доказательству универсальности. Данный подход не только приводит к доказательству универсальности для общих ансамблей Вигнера, но также предлагает концептуальное понимание того, как свойство универсальности возникает из некоторых простых принципов.

В пп. 1.2-1.7 мы даем обзор некоторых фактов, результатов и методов для случайных матриц Вигнера и некоторых других связанных с ними ансамблей. 
В этих пунктах содержится общая информация об основных понятиях. В п. 1.6 мы также объясняем ключевые новые идеи, перечисленные выше, и даем обзор различных результатов об универсальности. Читатель, желающий ознакомиться лишь с последними достижениями, может пропустить пп. 1.2-1.7 и сразу переходить к разделу 2 .

1.2. Ансамбли матриц Вигнера. Центральным вопросом теории вероятностей является универсальность кумулятивных статистик большого набора независимых данных. Для данного массива из $N$ независимых случайных величин

$$
\left(X_{1}, \ldots, X_{N}\right)
$$

можно образовать статистики, например, среднее и отклонение (флуктуацию)

$$
\bar{X}^{(N)}:=\frac{1}{N} \sum_{j=1}^{N} X_{j}, \quad S^{(N)}:=\frac{1}{\sqrt{N}} \sum_{j=1}^{N}\left(X_{j}-\mathrm{E} X_{j}\right) .
$$

При весьма общих условиях, при $N \rightarrow \infty$ наблюдается некоторое универсальное поведение: среднее сходится к своему математическому ожиданию, в частности, оно становится детерминированным,

$$
\bar{X}^{(N)} \rightarrow \lim _{N \rightarrow \infty} \mathrm{E} \bar{X}^{(N)}
$$

в предположении, что этот предел существует (закон больших чисел). Более того, отклонение $S^{(N)}$ сходится к нормальной гауссовой случайной величине $\xi$ с нулевым математическим ожиданием:

$$
S^{(N)} \rightarrow \xi \quad(\text { по распределению) }
$$

(центральная предельная теорема), т. е. функция плотности величины $\xi$ задается формулой $f(x)=(\sqrt{2 \pi} \sigma)^{-1} \exp \left(-x^{2} /\left(2 \sigma^{2}\right)\right)$. Дисперсия $\sigma^{2}$ величины $\xi$ равна среднему дисперсий величин $X_{j}$,

$$
\sigma^{2}:=\lim _{N \rightarrow \infty} \frac{1}{N} \sum_{j=1}^{N} \sigma_{j}^{2}, \quad \sigma_{j}^{2}:=\mathrm{E} g\left[X_{j}-\mathrm{E} X_{j}\right]^{2} .
$$

В частности, для независимых одинаково распределенных случайных величин значение $\bar{X}^{(N)}$ сходится к общему значению математического ожидания величин $X_{j}$, а $S^{(N)}$ сходится к нормальному распределению с нулевым средним и дисперсией, равной общей дисперсии величин $X_{j}$.

Возникновение одного общего универсального распределения - гауссова распределения - является замечательным фактом природы. Он показывает, что большие системы со многими независимыми компонентами ведут себя одинаковым образом, независимо от свойств распределения компонент.

Естественным образом возникает вопрос об обобщении свойства универсальности для массивов (1.8) на двумерные массивы, т. е. на матрицы

$$
X^{(N, M)}=\left(\begin{array}{cccc}
X_{11} & X_{12} & \ldots & X_{1 N} \\
X_{21} & X_{22} & \ldots & X_{2 N} \\
\vdots & \vdots & & \vdots \\
X_{M 1} & X_{M 2} & \ldots & X_{M N}
\end{array}\right)
$$


с независимыми матричными элементами. Рассматриваемые здесь статистики должны включать величины, отражающие матричный характер данных и зависящие от всех матричных элементов. Примером такой статистики является (евклидова) норма матрицы $X^{(N, M)}$. Несмотря на то что нормы различных случайных реализаций матрицы $X^{(N, M)}$ могут отличаться, известно, например, что в пределе при $N, M \rightarrow \infty$, при котором отношение $N / M$ стремится к фиксированному значению $d, 0<d \leqslant 1$, норма становится детерминированной, т. е. мы имеем [19], [20]

$$
\frac{1}{\sqrt{M}}\left\|X^{(N, M)}\right\| \rightarrow \sigma(1+\sqrt{d}) .
$$

Здесь предполагается, что все матричные элементы имеют нулевое математическое ожидание, $\mathrm{E} X_{i j}=0$, а их средняя дисперсия равна $\sigma^{2}$. Заметим, что типичная норма матрицы $X^{(N, M)}$ имеет порядок лишь $\sqrt{M}$, несмотря на то что матрица имеет размер $M \times N$ и состоит из элементов порядка $O(1)$. Если бы матричные элементы были сильно коррелированными, то норма была бы порядка $M$. Например, в случае, когда все матричные элементы одинаковы, $X_{i j}=X$, мы имеем $\left\|X^{(N, M)}\right\| \sim M$. Независимость матричных элементов предотвращает такой “сговор" и уменьшает типичное значение нормы матрицы в $\sqrt{M}$ раз, аналогично тому, что утверждает центральная предельная теорема (обратите внимание на нормировку $\sqrt{N}$ в (1.9)).

Изучение матриц приводит к намного более богатым структурам, чем только изучение их норм. При условии $M=N$ наиболее важными характеристиками квадратной матрицы являются ее собственные значения и собственные векторы. Как подсказывает формула (1.12), удобно считать, что матричные элементы имеют нулевое математическое ожидание, и умножить матрицу на $N^{-1 / 2}$, чтобы ее норма стала порядка 1. На протяжении большей части нашего обзора мы будем иметь дело с большими квадратными матрицами размера $N \times N$ вида

$$
H=H^{(N)}=\left(\begin{array}{cccc}
h_{11} & h_{12} & \ldots & h_{1 N} \\
h_{21} & h_{22} & \ldots & h_{2 N} \\
\vdots & \vdots & \ddots & \vdots \\
h_{N 1} & h_{N 2} & \ldots & h_{N N}
\end{array}\right)
$$

с матричными элементами, имеющими нулевое математическое ожидание:

$$
\mathrm{E} h_{i j}=0, \quad i, j=1, \ldots, N .
$$

Что касается нормировки, мы предполагаем, что матрица дисперсий

$$
\Sigma:=\left(\begin{array}{cccc}
\sigma_{11}^{2} & \sigma_{12}^{2} & \ldots & \sigma_{1 N}^{2} \\
\sigma_{21}^{2} & \sigma_{22}^{2} & \ldots & \sigma_{2 N}^{2} \\
\vdots & \vdots & \ddots & \vdots \\
\sigma_{N 1}^{2} & \sigma_{N 2}^{2} & \ldots & \sigma_{N N}^{2}
\end{array}\right), \quad \sigma_{i j}^{2}:=\mathrm{E}\left|h_{i j}\right|^{2},
$$

является дважды стохастической, т. е. для любого $i=1, \ldots, N$ мы имеем

$$
\sum_{j} \sigma_{i j}^{2}=\sum_{j} \sigma_{j i}^{2}=1 .
$$


Наиболее естественным примером является модель среднего поля, в которой

$$
\sigma_{i j}^{2}=\frac{1}{N}, \quad i, j=1,2, \ldots, N,
$$

т. е. каждый матричный элемент имеет размер $h_{i j} \sim N^{-1 / 2}$. Это соответствует стандартной матрице Вигнера. На протяжении большей части изложения читатель может ограничиться рассмотрением этого случая.

На случайные матрицы, как правило, накладываются некоторые ограничения типа симметрии. Например, мы будем рассматривать симметрические $\left(h_{i j}=h_{j i} \in \mathbb{R}\right)$ или эрмитовы $\left(h_{i j}=\bar{h}_{j i} \in \mathbb{C}\right)$ случайные матрицы. Как правило, мы будем предполагать, что матричные элементы независимы с учетом симметрии (в случае симметрических или эрмитовых матриц независимыми являются величины $\left.\left\{h_{i j}: i \leqslant j\right\}\right)$. Это приводит к следующему определению.

ОПРЕДЕЛЕНИЕ 1.1. Мы называем симметрическую или эрмитову случайную матрицу (1.13) размера $N \times N$ универсальной матрицей (ансамблем) Вигнера, если ее элементы имеют нулевое математическое ожидание (1.14), их дисперсии $\sigma_{i j}^{2}=\mathrm{E}\left|h_{i j}\right|^{2}$ удовлетворяют условию

$$
\sum_{j} \sigma_{i j}^{2}=1, \quad i=1, \ldots, N
$$

а величины $\left\{h_{i j}: i \leqslant j\right\}$ являются независимыми. Имеется важный подкласс универсальных ансамблей Вигнера, называемый обобщенными матрицами (ансамблями) Вигнера. Для таких матриц дополнительно требуется, чтобы дисперсии были сравнимыми, т. е. имели место неравенства

$$
0<C_{\mathrm{inf}} \leqslant N \sigma_{i j}^{2} \leqslant C_{\mathrm{sup}}<\infty, \quad i, j=1, \ldots, N,
$$

для некоторых фиксированных положительных констант $C_{\text {inf }}$ и $C_{\text {sup }}$. B специальном случае $\sigma_{i j}^{2}=1 / N$ мы получаем исходное определение матрии, Вигнера или ансамбля Вигнера [21].

Наиболее важными ансамблями Вигнера являются гауссов ортогоналъныи ансамбль (ГОА) и гауссов унитарный ансамбль (ГУА); т. е. симметрические или эрмитовы матрицы Вигнера, для которых нормированные матричные элементы $\sqrt{N} h_{i j}$ являются стандартными гауссовыми случайными величинами (в эрмитовом случае $\sqrt{N} h_{i j}$ - стандартными комплексными гауссовыми случайными величинами, т. е. Е $\left.\left|\sqrt{N} h_{i j}\right|^{2}=1\right)$.

В случае ансамбля Вигнера мы будем предполагать для простоты изложения, что величины $h_{i j}, i<j$, являются одинаково распределенными (т. е. совпадают не только их дисперсии). В этом случае мы зафиксируем некоторое распределение $\nu$ и будем предполагать, что нормированные матричные элементы $\sqrt{N} h_{i j}$ распределены в соответствии с $\nu$. В зависимости от типа симметрии, диагональные элементы могут иметь несколько иное распределение, но мы не будем принимать это во внимание в нашем обсуждении. Распределение $\nu$ будет называться распределением отдельных элементов матрицы $H$.

Иногда мы будем упоминать специальный класс универсальных матриц Вигнера, которые имеют ленточную структуру; они будут называться случайнъми 
ленточными матрицами. Их дисперсии задаются формулами

$$
\sigma_{i j}^{2}=W^{-1} f\left(\frac{[i-j]_{N}}{W}\right) \text {, }
$$

где $W \gg 1, f: \mathbb{R} \rightarrow \mathbb{R}_{+}$- ограниченная неотрицательная симметрическая функция с $\int f(x) \mathrm{d} x=1$, а число $[i-j]_{N} \in \mathbb{Z}$ определяется свойствами $[i-j]_{N} \equiv i-j \bmod N$ и $-N / 2<[i-j]_{N} \leqslant N / 2$. Заметим, что соотношение (1.16) выполняется лишь асимптотически при $W \rightarrow \infty$, но от этого можно избавиться путем соответствующей нормировки. Можно даже рассматривать $d$-мерные ленточные матрицы, в которых строки и столбцы помечены элементами конечной решетки $\Lambda \subset \mathbb{Z}^{d}$, а значения $\sigma_{i j}^{2}$ зависят лишь от разности $i-j$ для любых $i, j \in \Lambda$.

Другим классом случайных матриц, которые были известны еще до Вигнера, являются случайные ковариационные матрицы. Эти матрицы имеют вид

$$
H=X^{*} X
$$

где $X$ - прямоугольная матрица размера $M \times N$ вида (1.11), элементами которой являются независимые одинаково распределенные величины с нулевым математическим ожиданием и дисперсией $\mathrm{E}\left|X_{i j}\right|^{2}=M^{-1}$. Заметим, что элементы матрицы $H$ не являются независимыми, но они получаются простым способом из независимых элементов матрицы $X$. Эти матрицы возникают в статистических выборках и были впервые рассмотрены Уишартом [22]. В случае, когда величины $X_{i j}$ являются гауссовыми с нулевым математическим ожиданием, случайные ковариационные матрицы называются матрищами или ансамблями Уищарта.

1.3. Мотивации: от операторов Шрёдингера к $\zeta$-функциям. Мы будем заниматься в основном изучением статистик собственных значений больших случайных матриц, хотя результаты о случайных векторах также будут упоминаться. Основной физической мотивацией является тот факт, что случайные матрицы могут моделировать гамильтонов оператор для неупорядоченной квантовой системы. Свойства симметрии матрицы $H$ возникают из этого рассмотрения: симметрические матрицы представляют гамильтонианы систем, инвариантных относительно обращения времени (например, без магнитного поля), а эрмитовы матрицы соответствуют системам без симметрии обращения времени. (Имеется третий класс матриц - кватернионные самодвойственные матрицы, наиболее известной моделью которых является гауссов симплектический ансамбль (ГСА). Этот класс описывает системы с нечетным спином и без вращательной симметрии, но мы не будем подробно обсуждать его здесь.)

Случайные матрицы были изначально введены Э. Вигнером для моделирования собственных значений неизвестного гамильтониана тяжелых ядер; в отсутствие всякой информации он предположил, что матричные элементы являются независимыми одинаково распределенными случайными величинами с условием эрмитовости. Его весьма смелое видение вопроса заключалось 
в том, что хотя такое грубое приближение не сможет предсказать индивидуальные уровни энергии (собственные значения) ядер, статистические свойcmва этого приближения могут быть характеристиками некоторого глобального свойства ядер. Путем сравнения данных измерений уровней энергии ядер с численными вычислениями собственных значений некоторых случайных матриц он установил, что статистики уровней, т. е. распределение энергий перехода между соседними энергетическими уровнями (собственными значениями), демонстрируют замечательное совпадение и устойчивость. В частности, он заметил, что уровни энергии имеют тенденцию отталкивать друг друга, что весьма отличается от свойств статистик уровней вполне некоррелированных случайных точек (точечных пуассоновых процессов). Аналогичное свойство было обнаружено для случайных матриц: даже матрицы Вигнера, которые являются "максимально возможно стохастическими", дают наборы сильно коррелированных (отталкивающихся) собственных значений. Эта корреляция происходит из фундаментальных свойств симметрии матричного ансамбля; в частности, было обнаружено, что симметрические и эрмитовы матрицы имеют разную меру отталкивания уровней, в то время как в пределах одного класса симметрии имеет место универсальное поведение. Более подробное изложение истории этого замечательного открытия можно найти в [1].

Существует убеждение, что свойство универсальности для локальных статистик собственных значений имеет место для намного более широкого класса ансамблей матриц, чем введенные выше. Нет причины считать, что матричные элементы гамильтониана тяжелых ядер действительно представляют собой независимые одинаково распределенные случайные величины. Возможно, для универсальности и не требуется, чтобы матричные элементы были вполне независимыми или одинаково распределенными. Мало известно о матрицах с коррелирующими элементами, помимо унитарно инвариантных ансамблей (п. 1.5.3), в которых имеется корреляция весьма специального вида. В случае некоторого класса матриц Вигнера со слабо коррелированными элементами в [23], [24] был доказан полукруговой закон и его гауссова флуктуация.

Намного лучше изучены различные классы случайных матриц с независимыми, но не одинаково распределенными элементами. Наиболее хорошо известным примером является модель тесных связей Андерсона (Anderson tight binding model) [25], т. е. оператор Шрёдингера $-\Delta+\lambda V$ на регулярной квадратной решетке $\mathbb{Z}^{d}$ со случайным потенциалом $V$ и силой разупорядочивания $\lambda$. Эта модель описывает распространение электронов (проводимость) в ионной решетке с неупорядоченной средой. Будучи ограниченной на конечный параллелепипед, данная модель может быть представлена матрицей, диагональные элементы которой являются независимыми одинаково распределенными случайными величинами; детерминированные внедиагональные элементы задаются лапласианом.

Общая формулировка гипотезы универсальности для случайных операторов Шрёдингера утверждает, что имеется два различных режима в зависимости от энергии и силы разупорядочивания. В режиме сильного беспорядка собственные функции локализованы и локальные спектральные статистики являются пуассоновыми. В режиме слабого беспорядка собственные функции делокализованы и локальные статистики совпадают со статистиками гауссо- 
ва ансамбля матриц. Другие гипотезы, которые здесь обсуждаться не будут, связывают эти два режима с хаотическим или интегрируемым поведением соответствующей классической динамической системы. В соответствии с гипотезой Берри-Табора [26], пуассоновы статистики собственных значений должны возникать из квантизации классических интегрируемых динамик, в то время как теория случайных матриц возникает из квантизации хаотических динамических систем [27].

Возвращаясь к более конкретной модели Андерсона, для размерностей пространства три или выше и для слабой случайности имеется гипотеза, что эта модель описывает переход "металл-изолирующее вещество", т. е. в размерностях $d \geqslant 3$ собственные функции оператора $-\Delta+\lambda V$ делокализованы для малых $\lambda$ и локализованы для больших $\lambda$. Обоснование этого перехода является фундаментальным вопросом математики.

Режим локализации при большом беспорядке или вблизи границ спектра был хорошо изучен Фрёлихом и Спенсером при помощи техники мультимасштабирования (multiscale analysis) [28], [29], а затем Айземаном и Молчановым методом дробных моментов [30]; с тех пор в этой области появилось много работ. В частности, установлено, что локальные статистики собственных значений являются пуассоновыми [31] и что собственные функции экспоненциально локализуются, причем верхняя граница длины локализации расходится при приближении параметра энергии к заданной точке фазового перехода [32], [33].

Прогресс в изучении режима делокализации был гораздо более медленным. В случае решетки Бете, соответствующей бесконечномерному случаю, свойство делокализации был установлено в [34]-[36] (статистики собственных значений здесь являются пуассоновыми, в видимом противоречии с общими гипотезами, однако они являются таковыми по вполне определенной и хорошо понятной причине [37]). В конечных размерностях имеются лишь частные результаты. Существование абсолютно непрерывного спектра (т. е. расширенных состояний) было установлено в случае быстро убывающего потенциала, соответствующего режиму рассеяния [38]-[40]. Свойство диффузии было установлено для тяжелых квантовых частиц, погруженных в фотонное поле, в размерностях $d \geqslant 4$ [41]. Для исходного гамильтониана Андерсона с малой константой спаривания $\lambda$ собственные функции имеют длину локализации по крайней меpe $\lambda^{-2}$ [42]. Пространственный и временной масштаб $\lambda^{-2}$ соответствует кинетическому режиму, в котором квантовая эволюция может быть описана линейным уравнением Больцмана [43], [44]. Вне этого временного масштаба динамика является диффузной. Это было установлено в пределе масштаба $\lambda \rightarrow 0$ вплоть до времен порядка $t \sim \lambda^{-2-\kappa}$ с явно задаваемой константой $\kappa>0$ в [45].

Строгие результаты о локальных спектральных статистиках для модели Андерсона в режиме беспорядка отсутствуют, но имеется гипотеза (подтверждаемая многочисленными аргументами в физической литературе, особенно на основе суперсимметрических методов, см. [46]) о том, что локальные корреляционные функции собственных значений модели Андерсона конечного объема согласуются со статистиками ГОА в термодинамическом пределе. Статистики ГУА предположительно возникают в присутствие дополнительного магнитного поля, которое разрушает симметрию обращения времени в гамильтониане Андерсона. Основываясь на этой гипотезе, можно применять статистики 
собственных значений для численного вычисления фазовых диаграмм. Замечательным фактом является то, что случайный оператор Шрёдингера, задаваемый весьма разреженной случайной матрицей, представляет тот же самый класс универсальности, что и полная матрица Вигнера, по крайней мере в определенных пределах значения энергии.

Ввиду того что матрицы Вигнера имеют характер среднего поля, их намного проще изучать, чем модель Андерсона, и они всегда находятся в режиме делокализации. В этом обзоре мы в основном работаем с матрицами Вигнера, но также принимаем во внимание исходные мотивации для общих неупорядоченных систем. В частности, мы будем изучать не только статистики собственных значений, но также и собственные векторы, для которых будет установлена их полная делокализованность [16]. Локальные спектральные статистики внутри спектра являются универсальными, т. е. согласуются со статистиками соответствующего гауссова ансамбля (ГОА, ГУА, ГСА), в зависимости от типа симметрии матрицы. Этот вопрос будет главной целью нашего повествования.

Заканчивая этот пункт, мы упомянем еще некоторые направления исследований, которые далее рассматриваться не будут. Этот список не является полным.

Естественным промежуточным классом ансамблей между вполне стохастическими матрицами Вигнера и моделью Андерсона с диагональной случайностью является семейство случайных ленточных матрии. Они представляют собой эрмитовы или симметрические случайные матрицы $H$ с независимыми, но не одинаково распределенными элементами. Дисперсия $h_{i j}$ зависит лишь от $|i-j|$ и становится пренебрежимо малой, если значение $|i-j|$ превышает данный параметр $W$ - ширину ленты; например, $\sigma_{i j}^{2}=\mathrm{E}\left|h_{i j}\right|^{2} \sim \exp (-|i-j| / W)$. Имеется гипотеза [47], что система полностью делокализуется, если $W \gg \sqrt{N}$, а в противном случае длина локализации равна $W^{2}$. Более того, для узких лент, $W \ll \sqrt{N}$, локальные статистики собственных значений, по-видимому, являются пуассоновыми, в то время как для широких лент, $W \gg \sqrt{N}$, они должны задаваться статистиками ГУА или ГОА, в зависимости от класса симметрии. Свойства локализации для матриц $H$ при $W \ll N^{1 / 8}$ и верхняя оценка порядка $O\left(W^{8}\right)$ для длины локализации были установлены Дж. Шенкером [48], но этот результат не включает локальные статистики. Что касается свойств делокализации, в совместной работе с А. Ноулесом [49], [50] нами недавно была установлена диффузность в пределах времени до $t \ll W^{1 / 3}$, откуда вытекает, что длина локализации равна по крайней мере $W^{1+1 / 6}$.

Отметим, что свойство универсальности локальных статистик собственных значений широко изучалось в физической литературе при помощи техники суперсимметрии. Это дает весьма мощный метод получения результатов при помощи вычислений в седловых точках, однако аналитические рассуждения для приближений в седловых точках по-прежнему не являются математически строгими. На данный момент лишь плотность состояний была строго исследована при помощи этой техники [51]. Кроме того, квантовая диффузия также может быть исследована суперсимметрическими методами, и некоторые промежуточные модели могут быть строго проанализированы [52], [53].

Наконец, отметим, что хотя мы в основном описывали физические мотивировки, происходящие из неупорядоченных квантовых систем, случайные 
матрицы возникают также и во многих других областях физики и математики. Случайные матрицы являются фундаментальным объектом природы с очень богатой структурой. Наиболее замечательными являются взаимосвязи с $\zeta$-функцией. Имеется гипотеза о том, что нули $\zeta$-функции Римана $\zeta(s):=$ $\sum_{n=1}^{\infty} n^{-s}$, лежащие на вертикальной оси $\operatorname{Re} s=1 / 2$, имеют те же локальные статистики, что и ГУА (после соответствующей перенормировки). Обзор этой тематики и дальнейшие ссылки на обширные численные подтверждения этой гипотезы можно найти в классической книге Меты [1].

1.4. Плотность собственных значений и делокализация. Для данной симметрической или эрмитовой матрицы $H$ мы будем обозначать ее собственные значения через $\lambda_{1} \leqslant \lambda_{2} \leqslant \cdots \leqslant \lambda_{N}$. Они образуют случайный точечный процесс на вещественной прямой, распределение которого определяется общим вероятностным законом для матричных элементов. Так как функциональные соотношения между матричными элементами и собственными значениями являются в высшей степени нетривиальными, обычная мера произведения для матричных элементов порождает сложную и сильно коррелированную меру для собственных значений. Наша главная цель - понять, как устроена эта индуцированная мера.

При выбранной нормировке (1.16) типичный размер собственных значений имеет порядок 1. Далее мы докажем более точное утверждение, но пока полезно получить грубое представление о размере, вычислив значение $\operatorname{Tr} H^{2}$ двумя различными способами:

$$
\sum_{i=1}^{N} \lambda_{i}^{2}=\operatorname{Tr} H^{2}=\sum_{i, j=1}^{N}\left|h_{i j}\right|^{2}
$$

Переходя к математическим ожиданиям и используя (1.16), мы получаем

$$
\frac{1}{N} \sum_{i} \mathrm{E} \lambda_{i}^{2}=\frac{1}{N} \sum_{i, j} \sigma_{i j}^{2}=1
$$

т. е. в среднем мы имеем $\mathrm{E} \lambda_{i}^{2}=1$.

1.4.1. Полукруговой закон Вигнера и другие канонические плотности. Эмпирическое распределение собственных значений следует некоторой общей закономерности, называемой полукруговым законом Вигнера. Чтобы сформулировать этот закон, заметим, что типичное расстояние между соседними собственными значениями имеет порядок $1 / N$, так что внутрь фиксированного отрезка $[a, b] \subset \mathbb{R}$ попадает макроскопически много (порядка $N$ ) собственных значений. Более точно, можно показать (первое доказательство было дано Вигнером [21]), что для любых действительных чисел $a \leqslant b$ справедливы равенства

$$
\lim _{N \rightarrow \infty} \frac{1}{N} \#\left\{i: \lambda_{i} \in[a, b]\right\}=\int_{a}^{b} \varrho_{\mathrm{sc}}(x) \mathrm{d} x, \quad \varrho_{\mathrm{sc}}(x):=\frac{1}{2 \pi} \sqrt{\left(4-x^{2}\right)_{+}},
$$

где $(a)_{+}:=\max \{a, 0\}-$ положительная часть числа $a$. Отметим, что появляется универсальная плотность - полукруговой закон, который не зависит от свойств распределения матричных элементов. 
Полукруговой закон является характеристикой универсальных матриц Вигнера (см. определение 1.1). Для произвольных квадратных матриц с независимыми элементами, но без симметрии (т. е. когда $h_{i j}$ независимы для всех $i, j$ ), возникает аналогичная универсальная закономерность - закон окружностей. Например, если $h_{i j}$ являются независимыми одинаково распределенными случайными величинами с нулевым математическим ожиданием и общей дисперсией $\sigma_{i j}^{2}=N^{-1}$, то эмпирическая плотность собственных значений сходится к равномерной мере на единичном диске в комплексной плоскости [54]. Если же опустить условие независимости, то можно получить различные виды плотности.

Например, для случайных ковариационных матриц (1.19) эмпирическая плотность собственных значений $\lambda_{i}$ матрицы $H$ сходится к закону МарченкоПастура [19] в пределе при $M, N \rightarrow \infty$, при котором отношение $d=N / M$ остается фиксированным и $0 \leqslant d \leqslant 1$ :

$$
\begin{gathered}
\lim _{N \rightarrow \infty} \frac{1}{N} \#\left\{i: \lambda_{i} \in[a, b]\right\}=\int_{a}^{b} \varrho_{\mathrm{MP}}(x) \mathrm{d} x \\
\varrho_{\mathrm{MP}}(x):=\frac{1}{2 \pi d} \sqrt{\frac{\left[\left(\lambda_{+}-x\right)\left(x-\lambda_{-}\right)\right]_{+}}{x^{2}}}
\end{gathered}
$$

где $\lambda_{ \pm}:=(1 \pm \sqrt{d})^{2}$ - границы спектра. Заметим, что в случае $M \leqslant N$ матрица $H$ имеет макроскопически много нулевых собственных значений. Действительно, иначе бы спектры матриц $X X^{*}$ и $X^{*} X$ совпадали и закон Марченко-Пастура можно было бы применить к ненулевым собственным значениям, поменяв местами числа $M$ и $N$.

1.4.2. Метод моментов. Плотность собственных значений обычно изучается при помощи достаточно грубого метода моментов (см. обзор [3]); этот метод также использовался Вигнером в его доказательстве полукругового закона [21]. Например, для эрмитовых матриц Вигнера метод заключается в вычислении следов больших степеней матрицы $H$, т. е.

$$
\mathrm{E} \operatorname{Tr} H^{2 k}
$$

путем представления произведения в виде

$$
\mathrm{E} \sum_{i_{1}, i_{2}, \ldots, i_{2 k}} h_{i_{1} i_{2}} h_{i_{2} i_{3}} \cdots h_{i_{2 k} i_{1}} .
$$

Далее замечается, что каждому множителю $h_{x y}$ должен соответствовать некоторый $h_{y x}=\bar{h}_{x y}$, так как иначе значение математического ожидания будет равно нулю. Возможные последовательности индексов, соответствующие этим условиям спаривания, можно классифицировать по их сложности, и оказывается, что главный вклад дают так называемые возвращающиеся пути. Они представляют собой последовательности индексов $i_{1} i_{2} i_{3} \ldots i_{2 k} i_{1}$, возвращающиеся к начальному индексу $i_{1}$, которые можно производить при помощи закона подстановки

$$
a \rightarrow a b a, \quad b \in\{1, \ldots, N\}, \quad b \neq a,
$$


с произвольным индексом $b$. Очевидно, что такие последовательности индексов удовлетворяют условию спаривания, и оказывается, что именно они включают наибольшее возможное число $\left(N^{k}\right)$ независимых индексов. Число возвращающихся путей явно задается числами Каталана $C_{k}=\frac{1}{k+1}\left(\begin{array}{c}2 k \\ k\end{array}\right)$, так что значение $\mathrm{E} \operatorname{Tr} H^{2 k}$ можно вычислить достаточно точно для любого конечного $k$ :

$$
\frac{1}{N} \mathrm{E} \operatorname{Tr} H^{2 k}=\frac{1}{k+1}\left(\begin{array}{c}
2 k \\
k
\end{array}\right)+O_{k}\left(N^{-2}\right) .
$$

Заметим, что число независимых индексов $\left(N^{k}\right)$ в точности сокращается с размером $k$-кратного произведения дисперсий $\left(\mathrm{E}|h|^{2}\right)^{k}=N^{-k}$. Если распределение матричных элементов симметрично, то следы нечетных степеней обращаются в нуль, так как они никак не могут удовлетворять условию спаривания. Без условия симметричности следы нечетных степеней являются не нулевыми, но пренебрежимо малыми.

Мы вычислим след резольвенты, или так называемое преобразование Cmuлтьеса для эмпирической плотности

$$
\varrho_{N}(\mathrm{~d} x):=\frac{1}{N} \sum_{j=1}^{N} \delta\left(x-\lambda_{j}\right)
$$

собственных значений, т. е. определим

$$
m(z)=m_{N}(z):=\frac{1}{N} \operatorname{Tr} \frac{1}{H-z}=\frac{1}{N} \sum_{j=1}^{N} \frac{1}{\lambda_{j}-z}=\int_{\mathbb{R}} \frac{\mathrm{d} \varrho_{N}(x)}{x-z}
$$

для всех $z=E+i \eta, E \in \mathbb{R}, \eta>0$. При больших $z$ функция $m_{N}$ раскладывается следующим образом:

$$
m_{N}(z)=\frac{1}{N} \operatorname{Tr} \frac{1}{H-z}=-\frac{1}{N z} \sum_{m=0}^{\infty}\left(\frac{H}{z}\right)^{m} .
$$

Таким образом, вычисляя математическое ожидание с использованием (1.22) и пренебрегая поправочными членами, мы получаем

$$
\mathrm{E}_{N}(z) \approx-\sum_{m=0}^{\infty} \frac{1}{k+1}\left(\begin{array}{c}
2 k \\
k
\end{array}\right) z^{-(2 k+1)},
$$

что путем некоторых вычислений можно отождествить со степенным рядом функции $\left(-z+\sqrt{z^{2}-4}\right) / 2$. Данное приближение становится точным в пределе при $N \rightarrow \infty$. Разложение (1.24) возможно лишь при больших $z$, однако ввиду того, что предел является аналитической функцией от $z$, соотношение

$$
\lim _{N \rightarrow \infty} \operatorname{E} m_{N}(z)=\frac{1}{2}\left(-z+\sqrt{z^{2}-4}\right)
$$

можно аналитически продолжить на всю верхнюю полуплоскость $z=E+i \eta$, $\eta>0$. В качестве простого упражнения предлагается убедиться, что результатом аналитического продолжения является в точности преобразование Стилтьеса плотности полукруга, т. е.

$$
m_{\mathrm{sc}}(z):=\frac{1}{2}\left(-z+\sqrt{z^{2}-4}\right)=\int_{\mathbb{R}} \frac{\varrho_{\mathrm{sc}}(x) \mathrm{d} x}{x-z} .
$$


Функция квадратного корня выбирается с разрезами ветвления внутри отрезка $[-2,2]$, так что $\sqrt{z^{2}-4} \sim z$ вблизи бесконечности. Это обеспечивает условие $\operatorname{Im} m_{\mathrm{sc}}(z)>0$ при $\operatorname{Im} z>0$. Так как преобразование Стилтьеса задает меру однозначно, а поточечная сходимость преобразований Стилтьеса влечет слабую сходимость мер, мы получаем

$$
\mathrm{Ed} \varrho_{N}(x) \rightarrow \varrho_{\mathrm{sc}}(x) \mathrm{d} x .
$$

Ненамного больше усилий требуется для доказательства того, что равенство

$$
\lim _{N \rightarrow \infty} m_{N}(z)=\frac{1}{2}\left(-z+\sqrt{z^{2}-4}\right)
$$

имеет место с большой вероятностью, т. е. мы имеем сходимость по вероятности, а не только сходимость в среднем. Подробности можно найти в [3].

1.4.3. Локальный полукруговой закон. Метод моментов, как правило, позволяет определить резольвенту для любого фиксированного $z$ и тем самым приводит к полукруговому закону в слабом пределе, т. е. закон (1.20) справедлив для любого фиксированного отрезка $I:=[a, b]$ при $N \rightarrow \infty$. При этом фиксированный отрезок $I$ длины $|I|$ как правило содержит порядка $N|I|$ собственных значений. Возникает естественный вопрос о том, выполняется ли полукруговой закон также и локально, т. е. для отрезков, которые могут сжиматься с увеличением $N$, но с выполнением соотношения $N|I| \gg 1$. В самом деле, полукруговой закон является разновидностью закона больших чисел, который требует лишь того, чтобы число рассматриваемых случайных объектов стремилось к бесконечности. Так как имеет место формула

$$
\operatorname{Im} m_{N}(z)=\frac{1}{N} \sum_{i=1}^{N} \frac{\eta}{\left(\lambda_{i}-E\right)^{2}+\eta^{2}} \sim \frac{\pi}{N} \sum_{i=1}^{N} \delta_{\eta}\left(\lambda_{i}-E\right), \quad z=E+i \eta,
$$

где $\delta_{\eta}$ обозначает приближение дельта-функции размера $\eta$, мы видим, что знание преобразования Стилтьеса для некоторого $z \in \mathbb{C}$ с $\operatorname{Im} z=\eta$ в сущности эквивалентно знанию локальной плотности в масштабе $\eta$, т. е. в интервале длины $|I| \sim \eta$.

В [55]-[57] нами было доказано, что локальный полукруговой закон выполняется для наименьшего возможного порядка величин $\eta \gg 1 / N$. Другими словами, соотношение (1.20) выполнено, даже если длина отрезка $I=[a, b]$ имеет порядок $1 / N$, т. е. типично содержит только очень большое, но конечное число собственных значений. Это послужило основным техническим средством для последующего изучения локальных спектральных статистик. Имеется несколько вариантов локального полукругового закона; мы приведем три точных утверждения: теорема 1.9 (из [57]), теорема 2.5 (из [8]) и теорема 2.19 (из [7]).

Метод доказательства этих утверждений отличается от метода моментов, но по-прежнему использует резольвенты или преобразование Стилтьеса. Ключевое наблюдение (см. также предыдущие работы, например, [58], [19]) заключается в том, что преобразование Стилтьеса $m_{\mathrm{sc}}(z)$ плотности полукруга $\varrho_{\mathrm{sc}}$ удовлетворяет следующему простому квадратному уравнению:

$$
m_{\mathrm{sc}}(z)+\frac{1}{z+m_{\mathrm{sc}}(z)}=0
$$


причем $m_{\mathrm{sc}}(z)$ выбирается из двух возможных решений по правилу, описанному после формулы (1.26). Стратегия заключается в том, что путем разложения эмпирического преобразования Стилтьеса $m_{N}(z)(1.23)$ по минорам матрицы $H$ мы доказываем, что $m_{N}$ удовлетворяет самосогласованному уравнению (1.29) приблизительно и с большой вероятностью:

$$
m_{N}(z)+\frac{1}{z+m_{N}(z)} \approx 0
$$

Далее мы завершаем доказательство соотношения $m_{N} \approx m_{\mathrm{sc}}$, используя стабильность уравнения (1.29). Так как стабильность разрушается вблизи границ, т. е. при $E=\operatorname{Re} z \approx \pm 2$, то оценка вблизи границ будет слабее, что указывает на большую флуктуацию собственных значений на границе.

Наши лучшие результаты в этом направлении получены в [7] (эта работа также содержит результаты работ [9], [8] в более упорядоченном виде). В [7] было показано, что не только след функции Грина (1.23), но и индивидуальные диагональные элементы задаются полукруговым законом. Эти результаты уже обсуждались неформально в п. 1.1.1, где мы отмечали, что они применимы также и для универсальных матриц Вигнера (см. теоремы 2.5 и 2.19).

Для универсальных ансамблей Вигнера в работах Гионне [59] и АндерсонаЦайтуни [60] было доказано, что плотность собственных значений сходится к полукруговому закону Вигнера в большом масштабе; наш результат показывает, что это также справедливо в малом масштабе. Например, для ленточных матриц (определение см. в (1.18)) с шириной ленты $W$ мы получаем, что полукруговой закон соблюдается вплоть до значений энергии порядка $1 / W$. Показано, что длина делокализации по крайней мере не меньше, чем ширина ленты $W$. Отметим, что некоторые трехмерные аналоги гауссовых ленточных матриц рассматривались Дисертори, Пинсоном и Спенсером [51] с использованием суперсимметричных методов. Они доказали, что математическое ожидание плотности собственных значений является гладкой функцией и задается полукруговым законом Вигнера с точностью, определяемой шириной ленты.

1.4.4. Плотность собственных значений для инвариантных ансамблей. Помимо прямого указания заданного вероятностного закона $\nu$ для матричных элементов, имеется другой естественный способ задания распределения вероятностей для симметрических или эрмитовых матриц. Этот способ заключается в задании функции плотности непосредственно на множестве матриц:

$$
\mathscr{P}(H) \mathrm{d} H:=Z^{-1} \exp (-N \operatorname{Tr} V(H)) \mathrm{d} H .
$$

Здесь $\mathrm{d} H=\prod_{i \leqslant j} \mathrm{~d} H_{i j}-$ плоская мера Лебега (если матрицы эрмитовы и $i<j$, то $\mathrm{d} H_{i j}$ является мерой Лебега на комплексной плоскости $\mathbb{C}$ ). Для того чтобы мера, определяемая формулой (1.30), была конечной, нужно предположить, что функция $V: \mathbb{R} \rightarrow \mathbb{R}$ растет не слишком быстро на бесконечности (логарифмического роста достаточно); здесь $Z$ является нормирующим множителем. Распределения вероятностей вида (1.30) называются инвариантными ансамблями, так как они инвариантны относительно ортогональных или унитарных сопряжений (в случае симметрических или эрмитовых матриц соответственно). 
Например, в эрмитовом случае для любой фиксированной унитарной матрицы $U$ преобразование

$$
H \rightarrow U^{*} H U
$$

не меняет распределения (1.30), благодаря тождеству $\operatorname{Tr} V\left(U^{*} H U\right)=\operatorname{Tr} V(H)$.

Матрицы Вигнера и инвариантные ансамбли - это два разных мира, для исследования которых используются совершенно различные математические инструменты. В действительности, эти два класса практически не пересекаются; единственными инвариантными матрицами Вигнера являются гауссовы ансамбли. Это утверждение содержится в следующей лемме (см. [2] или теорему 2.6.3 в [1]).

Лемма 1.2. Пусть симметрический или эрмитов матричный ансамбль, задаваемый формулой (1.30), имеет независимые элементы $h_{i j}, i \leqslant j$. Тогда $V(x)$ представляет собой многочлен второй степени, т.е. $V(x)=a x^{2}+$ $b x+c$, причем $a>0$. Это означает, что по модулю тривиальных сдвигов и нормировки, данный ансамбль есть ГОА или ГУА.

Плотность собственных значений инвариантного ансамбля (1.30) определяется некоторой вариационной задачей [2]. Она задается равновесной плотностью газа с логарифмическим взаимодействием с собой и внешним потенциалом $V$, т. е. решением задачи

$$
\inf _{\varrho}\left\{\int_{\mathbb{R}} \int_{\mathbb{R}} \log |s-t|^{-1} \varrho(\mathrm{d} s) \varrho(\mathrm{d} t)+\int V(t) \varrho(\mathrm{d} t)\right\},
$$

где точная нижняя грань берется по всем вероятностным мерам $\varrho$. При некоторых достаточно слабых условиях на $V$ равновесная мера абсолютно непрерывна, т. е. $\varrho_{\text {eq }}(\mathrm{d} t)=\varrho_{\mathrm{eq}}(t) \mathrm{d} t$, и имеет компактный носитель. Если $V$ является многочленом, то носитель состоит из конечного числа отрезков. Эмпирическая плотность собственных значений сходится к $\varrho_{\mathrm{eq}}$ в смысле $(1.20)$, где $\varrho_{\mathrm{sc}}$ заменяется на функцию $\varrho_{\text {eq. }}$ Несложно проверить, что решением этой вариационной задачи в гауссовом случае $V(x)=x^{2} / 2$ действительно является функция $\varrho_{\mathrm{sc}}$.

1.4.5. Делокализация собственных векторов. Наряду со статистиками собственных значений, можно также изучать собственные векторы случайных матриц. В свете гипотезы об универсальности для неупорядоченных систем, обсуждавшейся в п. 1.3, проверка этой гипотезы также и на уровне собственных векторов представляется весьма интересной задачей. Матрицы Вигнера являются моделями среднего поля, и с точки зрения физической интуиции они всегда находятся в делокализованном режиме. Конечно, они по-прежнему являются конечными матрицами и поэтому не могут иметь абсолютно непрерывный спектр, который является наиболее стандартным проявлением делокализации для специалистов по случайным операторам Шрёдингера. Однако вопрос о делокализации собственных векторов также имеет смысл и для больших, но конечных матриц. Удивительным образом этот вопрос практически не рассматривался ни специалистами по теории случайных матриц, ни специалистами по случайным операторам Шрёдингера до того момента, как он недавно был поднят Т. Спенсером. Им было отмечено на одном из докладов, что в случае гауссовых ансамблей простое рассуждение с инвариантностью показывает, 
что собственные векторы $\mathbf{v} \in \mathbb{C}^{N}$ полностью делокализуются в том смысле, что их $\ell^{4}$-норма удовлетворяет соотношению $\|\mathbf{v}\|_{4} \sim N^{-1 / 4}$ (при условии $\|\mathbf{v}\|_{2}=1$ ). Это свидетельствует о сильной делокализации, так как, с одной стороны, согласно неравенству Коши-Буняковского мы имеем

$$
N^{-1 / 2}\|\mathbf{v}\|_{2}=\left(\frac{1}{N} \sum_{i=1}^{N}\left|v_{i}\right|^{2}\right)^{1 / 2} \leqslant\left(\frac{1}{N} \sum_{i=1}^{N}\left|v_{i}\right|^{4}\right)^{1 / 4}=N^{-1 / 4}\|\mathbf{v}\|_{4},
$$

т. е. всегда выполняется неравенство $\|\mathbf{v}\|_{4} \geqslant N^{-1 / 4}$, а с другой стороны, это неравенство близко к равенству в случае, когда все координаты собственного вектора имеют одинаковый размер $\left|v_{i}\right| \sim N^{-1 / 2}$.

Это простое рассуждение с инвариантностью работает лишь в гауссовом случае, когда имеет место унитарная инвариантность. Возникает естественный вопрос, делокализуются ли собственные векторы произвольных ансамблей Вигнера. Ответ на этот вопрос положителен. Нами было доказано в [57; следствие 3.2], что для данного $\ell^{2}$-нормированного собственного вектора $\mathbf{v}$ матрицы Вигнера $H$ с собственным значением $\lambda$, которое не лежит вблизи границ спектра, т. е.

$$
H \mathbf{v}=\lambda \mathbf{v}, \quad \lambda \in[-2+\kappa, 2-\kappa],
$$

при некотором $\kappa>0$, имеется следующая оценка для $\ell^{p}$-нормы вектора $\mathbf{v}$ (для $2<p<\infty)$ :

$$
\|\mathbf{v}\|_{p} \leqslant Q N^{-1 / 2+1 / p}
$$

с очень высокой вероятностью: множество исключительных событий субэкспоненциально мало по $Q$ при больших $Q$. Аналогичная оценка с логарифмической поправкой существует также для $p=\infty$. Точное утверждение будет дано в теоремах 2.21 и 2.22. Это фактически является следствием из локального полукругового закона (теоремы 2.5), который неформально обсуждался в п. 1.4.3.

Заметим, что для $\ell^{2}$-нормированного собственного вектора $\mathbf{v}$ размер его $\ell^{p}$-нормы (при $p>2$ ) дает информацию о делокализации. Полная делокализация имеет место при $\|\mathbf{v}\|_{p} \lesssim N^{-1 / 2+1 / p}$, так как это соответствует $\ell^{p}$-норме вполне делокализованного вектора $\mathbf{v}=\left(N^{-1 / 2}, \ldots, N^{-1 / 2}\right)$. Напротив, вполне локализованный собственный вектор $\mathbf{v}=(0, \ldots, 0,1,0, \ldots, 0)$ имеет $\ell^{p}$-норму один.

1.5. Локальные статистики собственных значений: предыдущие результаты. Главным вопросом о случайных матрицах является гипотеза универсальности, согласно которой локальные статистики собственных значений больших квадратных $(N \times N)$-матриц $H$ определяются типом симметрии ансамбля и не зависят от других особенностей распределения. Оказывается, что локальные статистики обладают даже более сильными свойствами универсальности, чем плотность собственных значений.

Термин “локальные статистики" относится к наблюдаемым величинам, которые могут различать индивидуальные собственные значения. Для всех рассматриваемых до сих пор ансамблей мы использовали нормировку, при которой большинство собственных значений оставалось внутри компактного множества при $N \rightarrow \infty$; другими словами, предельная функция плотности $\varrho$ имела 
компактный носитель. В этом случае типичное расстояние между соседними собственными значениями имеет порядок $N^{-1}$. Это справедливо внутри спек$m p a$, т. е. на положительном расстоянии от грании, спектра. Границы спектра соответствуют точкам, в которых функция $\varrho$ стремится к нулю. Например, для распределения полукруга Вигнера $\varrho_{\mathrm{sc}}$ границы спектра суть точки \pm 2 , для распределения Марченко-Пастура (1.21) границами спектра являются $\lambda_{ \pm}$, а для некоторых инвариантных ансамблей носитель плотности собственных значений может состоять из нескольких отрезков, т. е. иметь более двух границ спектра.

1.5.1. Универсальность внутри спектра: синус-ядро и распределение промежутков. Для того чтобы определить собственные значения и их совместное распределение внутри спектра, необходимо "увеличить масштаб" точечного процесса собственных значений путем умножения на число $N$. Мы фиксируем два вещественных числа $\alpha_{1}, \alpha_{2}$ и энергию $E$, для которой $\varrho(E)>0$, и задаемся вопросом о нахождении такого значения вероятности, при котором имеется собственное значение в $E+\alpha_{1} /[N \varrho(E)]$ и одновременно имеется собственное значение в $E+\alpha_{2} /[N \varrho(E)]$ (нормировка выбрана таким образом, что типичное число собственных значений между этими точками не зависит от $E$ ). Оказывается, что ответ на этот вопрос не зависит о особенностей ансамбля и от энергии $E$, а зависит лишь от типа симметрии. Например, в эрмитовом случае нужное значение вероятности задается формулой

$$
\begin{gathered}
\mathrm{P}\left\{\text { имеются собственные значения } \lambda \in E+\frac{\alpha_{1}+\mathrm{d} \alpha_{2}}{N \varrho(E)} \text { и } \lambda^{\prime} \in E+\frac{\alpha_{2}+\mathrm{d} \alpha_{2}}{N \varrho(E)}\right\} \\
=\left[1-\left(\frac{\sin \pi\left(\alpha_{1}-\alpha_{2}\right)}{\pi\left(\alpha_{1}-\alpha_{2}\right)}\right)^{2}\right] \mathrm{d} \alpha_{1} \mathrm{~d} \alpha_{2} .
\end{gathered}
$$

Функция в правой части данной формулы получается из знаменитого синусядpa, и ее следует рассматривать как определитель $(2 \times 2)$-матрицы вида

$$
\operatorname{det}\left(K\left(\alpha_{i}-\alpha_{j}\right)\right)_{i, j=1}^{2}, \quad K(x):=\frac{\sin \pi x}{\pi x} .
$$

Явный вид ядра $K$ в симметрическом случае более сложный (см. [2]), но он универсален, а корреляционные функции имеют ту же структуру определителя.

Заметим, что формула (1.32) содержит намного больше информации о собственных значениях, чем полукруговой закон (1.20). Во-первых, эта формула предоставляет локальную информацию после увеличения до масштаба, при котором начинают играть роль индивидуальные собственные значения. Во-вторых, она выражает корреляцию между двумя собственными значениями. Например, ввиду того, что $\frac{\sin y}{y} \rightarrow 1$ при $y \rightarrow 0$, собственные значения отталкиваются друг от друга.

В общем случае $k$-е корреляционные функции (или $k$-точечные маргиналы) дают информацию о совместном поведении наборов из $k$ собственных значений. Их определение заключается в следующем.

ОПРЕДЕЛЕНИЕ 1.3. Пусть $p_{N}\left(\lambda_{1}, \ldots, \lambda_{N}\right)$ - совместное симметризованное распределение вероятностей собственных значений. Для каждого $k \geqslant 1$ 
$k$-точечная корреляционная функция определяется по формуле

$$
p_{N}^{(k)}\left(\lambda_{1}, \ldots, \lambda_{k}\right):=\int_{\mathbb{R}^{N-k}} p_{N}\left(\lambda_{1}, \ldots, \lambda_{k}, \lambda_{k+1}, \ldots, \lambda_{N}\right) \mathrm{d} \lambda_{k+1} \cdots \mathrm{d} \lambda_{N} .
$$

ЗАмечАниЕ. Мы, как правило, нумеруем собственные значения в порядке возрастания, но здесь мы не накладываем этого ограничения и рассматриваем $p_{N}\left(\lambda_{1}, \ldots, \lambda_{N}\right)$ как симметрическую функцию от $N$ переменных $\boldsymbol{\lambda}=$ $\left(\lambda_{1}, \ldots, \lambda_{N}\right)$ в $\mathbb{R}^{N}$. Наряду с этим можно рассматривать плотность $\tilde{p}_{N}(\boldsymbol{\lambda})=$ $N ! p_{N}(\boldsymbol{\lambda}) \cdot \mathbf{1}\left(\boldsymbol{\lambda} \in \Xi^{(N)}\right)$, где

$$
\Xi^{(N)}:=\left\{\lambda_{1}<\cdots<\lambda_{N}\right\} \subset \mathbb{R}^{N} .
$$

Важность $k$-точечных корреляционных функций объясняется тем, что они дают математические ожидания наблюдаемых (функций) $O$, зависящих от наборов $k$ собственных значений, по формуле

$$
\frac{(N-k) !}{N !} \mathrm{E} \sum_{i_{1}, \ldots, i_{k}=1}^{N} O\left(\lambda_{i_{1}}, \ldots, \lambda_{i_{k}}\right)=\int_{\mathbb{R}^{k}} O\left(x_{1}, \ldots, x_{k}\right) p_{N}^{(k)}\left(x_{1}, \ldots, x_{k}\right) \mathrm{d} x_{1} \cdots \mathrm{d} x_{k},
$$

где суммирование производится по всем различным индексам $i_{1}, \ldots, i_{k}$, а множитель в начале формулы нормирует сумму.

Например, одноточечная функция $p_{N}^{(1)}$ выражает плотность. В частности, выбирая в качестве наблюдаемой характеристическую функцию отрезка $[a, b]$, т. е. $O(x)=\mathbf{1}(x \in[a, b])$, мы получаем

$$
\frac{1}{N} \#\left\{i: \lambda_{i} \in[a, b]\right\}=\frac{1}{N} \sum_{i=1}^{N} O\left(\lambda_{i}\right)=\int O(x) p_{N}^{(1)}(x) \mathrm{d} x=\int_{a}^{b} p_{N}^{(1)}(x) \mathrm{d} x .
$$

Таким образом, полукруговой закон Вигнера (1.20) утверждает, что $p_{N}^{(1)}$ слабо сходится к $\varrho_{\text {sc }}$ при $N \rightarrow \infty$.

Универсальность синус-ядра в эрмитовом случае выражает тот факт, что (слабый) предел нормированной $k$-точечной корреляционной функции при $N \rightarrow \infty$ задается определителем функций $K(x)$ из (1.33), т. е.

$$
\frac{1}{[\varrho(E)]^{k}} p_{N}^{(k)}\left(E+\frac{\alpha_{1}}{N \varrho(E)}, \ldots, E+\frac{\alpha_{k}}{N \varrho(E)}\right) \rightarrow \operatorname{det}\left(K\left(\alpha_{i}-\alpha_{j}\right)\right)_{i, j=1}^{k}
$$

для любого фиксированного значения $E$, в смысле слабой сходимости функций от переменных $\left(\alpha_{1}, \ldots, \alpha_{k}\right)$.

Как только $k$-точечные корреляционные функции определены, легко вывести предельные теоремы и для других величин, связанных с индивидуальными собственными значениями. Наиболее интересной из них является распределение промежутков, т. е. распределение разностей соседних собственных значений, $\lambda_{j+1}-\lambda_{j}$. Заметим, что хотя это распределение, на первый взгляд, включает в себя только два собственных значения, оно не выражается через двухточечные корреляционные функции, так как два собственных значения должны быть последовательными. Тем не менее, распределение промежутков можно выразить через все корреляционные функции следующим образом. 
Фиксируем энергию $E$ с $|E|<2$. Для $s>0$ и для некоторого $N$-зависимого параметра $t$ с $1 / N \ll t \ll 1$ положим

$$
\begin{aligned}
\Lambda(s) & =\Lambda_{N}(s) \\
& :=\frac{1}{2 N t \varrho_{\mathrm{sc}}(E)} \#\left\{1 \leqslant j \leqslant N-1: \lambda_{j+1}-\lambda_{j} \leqslant \frac{s}{N \varrho_{\mathrm{sc}}(E)}, \| \lambda_{j}-E \mid \leqslant t\right\}
\end{aligned}
$$

- отношение количества разностей нормированных собственных значений, попадающих ниже порогового значения $s$, к количеству всех собственных значений в большой, но все же микроскопической окрестности энергии $E$. Пусть $\mathscr{K}_{\alpha}$ - оператор, действующий в пространстве $L^{2}((0, \alpha))$ с интегральным ядром $K(x, y):=\frac{\sin \pi(x-y)}{\pi(x-y)}$. Тогда для любого $E \mathrm{c}|E|<2$ и для любого $s>0$

$$
\lim _{N \rightarrow \infty} \mathrm{E} \Lambda_{N}(s)=\int_{0}^{s} p(\alpha) \mathrm{d} \alpha, \quad p(\alpha):=\frac{\mathrm{d}^{2}}{\mathrm{~d} \alpha^{2}} \operatorname{det}\left(1-\mathscr{K}_{\alpha}\right),
$$

где det - определитель Фредгольма оператора $1-\mathscr{K}_{\alpha}$ (заметим, что оператор $\mathscr{K}_{\alpha}$ - компактный). Функция плотности $p(s)$ расстояний между двумя ближайшими соседними собственными значениями ведет себя в очень хорошем, но не точном приближении (называемом оценкой Вигнера), как $p(s) \approx \pi s e^{-\pi s^{2} / 4} / 2$ в симметрическом случае и как $p(s) \approx 32 \pi^{-2} s^{2} e^{-4 s^{2} / \pi}$ в эрмитовом случае [1].

Заметим, что такое поведение резко контрастирует со статистиками расстояний между уровнями для пуассоновых точечных процессов, где соответствующая плотность есть $p(s)=e^{-s}$ (после такой нормировки процесса, при которой среднее расстояние равно единице). В частности, случайные матрицы демонстрируют отталкивание уровней, причем сила отталкивания зависит от класса симметрии (отметим различное поведение функций $p(s)$ при $s \approx 0$ ).

Для доказательства формулы (1.36) мы используем формулу включенияисключения, которая приводит к выражению

$$
\begin{aligned}
\mathrm{E} \Lambda(s)= & \frac{1}{2 N t \varrho} \sum_{m=2}^{\infty}(-1)^{m}\left(\begin{array}{l}
N \\
m
\end{array}\right) \int_{-t}^{t} \mathrm{~d} v_{1} \cdots \int_{-t}^{t} \mathrm{~d} v_{m} \mathbf{1}\left\{\max \left|v_{i}-v_{j}\right| \leqslant \frac{s}{N \varrho}\right\} \\
& \times p_{N}^{(m)}\left(E+v_{1}, \ldots, E+v_{m}\right)
\end{aligned}
$$

где $\varrho=\varrho_{\mathrm{sc}}(E)$. После замены переменных получаем

$$
\begin{aligned}
\mathrm{E} \Lambda(s)= & \frac{1}{2 N t \varrho} \sum_{m=2}^{\infty}(-1)^{m} \int_{-N \varrho t}^{N \varrho t} \mathrm{~d} z_{1} \cdots \int_{-N \varrho t}^{N \varrho t} \mathrm{~d} z_{m} \\
& \times\left(\begin{array}{l}
N \\
m
\end{array}\right) \frac{1}{(N \varrho)^{m}} p_{N}^{(m)}\left(u+\frac{z_{1}}{N \rho}, \ldots, u+\frac{z_{m}}{N \rho}\right) \mathbf{1}\left\{\max \left|z_{i}-z_{j}\right| \leqslant s\right\} \\
= & \frac{1}{2 N t \varrho} \sum_{m=2}^{\infty}(-1)^{m} m \int_{-N \varrho t}^{N \varrho t} \mathrm{~d} z_{1} \int_{0}^{s} \mathrm{~d} a_{2} \cdots \int_{0}^{s} \mathrm{~d} a_{m} \\
& \times\left(\begin{array}{l}
N \\
m
\end{array}\right) \frac{1}{(N \varrho)^{m}} p_{N}^{(m)}\left(u+\frac{z_{1}}{N \rho}, u+\frac{z_{1}+a_{2}}{N \rho}, \ldots, u+\frac{z_{1}+a_{m}}{N \rho}\right),
\end{aligned}
$$


где множитель $m$ возникает из рассмотрения сектора интегрирования $z_{1} \leqslant z_{j}$, $j \geqslant 2$. Переходя к пределу при $N \rightarrow \infty$ и используя (1.35), мы получаем

$$
\lim _{N \rightarrow \infty} \mathrm{E} \Lambda(s)=\sum_{m=2}^{\infty} \frac{(-1)^{m}}{(m-1) !} \int_{0}^{s} \mathrm{~d} a_{2} \cdots \int_{0}^{s} \mathrm{~d} a_{m} \operatorname{det}\left(\frac{\sin \pi\left(a_{i}-a_{j}\right)}{\pi\left(a_{i}-a_{j}\right)}\right)_{i, j=1}^{m}
$$

где в последнем определителе мы полагаем $a_{1}=0$. Возможность перестановки предела и суммирования легко устанавливается при помощи рассуждения с альтернированными рядами. Заметим, что левая часть формулы (1.39) имеет вид $\int_{0}^{s} p(\alpha) \mathrm{d} \alpha$, где $p(\alpha)$ - вторая производная определителя Фредгольма $\operatorname{det}\left(1-\mathscr{K}_{\alpha}\right)$ из (1.36) (подробности см. в [61] или в [3]). Таким образом, мы имеем

$$
\lim _{N \rightarrow \infty} \mathrm{E} \Lambda_{N}(s)=\int_{0}^{s} p(\alpha) \mathrm{d} \alpha .
$$

1.5.2. Универсальность на границе спектра: ядро Аири. Вблизи границ спектра и при другой нормировке возникает иной тип универсальности. Он также имеет вид определителя, но ядро задается ядром Аири

$$
A(x, y):=\frac{\operatorname{Ai}(x) \operatorname{Ai}^{\prime}(y)-\operatorname{Ai}^{\prime}(x) \operatorname{Ai}(y)}{x-y},
$$

где $\operatorname{Ai}(x)$ обозначает функцию Аири, т. е.

$$
\operatorname{Ai}(x)=\frac{1}{\pi} \int_{0}^{\infty} \cos \left(\frac{1}{3} t^{3}+x t\right) \mathrm{d} t
$$

Эта функция представляет собой решение дифференциального уравнения $y^{\prime \prime}-x y=0$ второго порядка, с нулевым граничным условием при $x=\infty$. Имеется результат, аналогичный сходимости (1.35), о том, для верхней границы спектра $E=2$ эрмитовых матриц Вигнера имеет место следующий слабый предел при $N \rightarrow \infty$ :

$$
p_{N}^{(k)}\left(2+\frac{\alpha_{1}}{N^{2 / 3}}, 2+\frac{\alpha_{2}}{N^{2 / 3}}, \ldots, 2+\frac{\alpha_{k}}{N^{2 / 3}}\right) \rightarrow \operatorname{det}\left(A\left(\alpha_{i}, \alpha_{j}\right)\right)_{i, j=1}^{k} .
$$

Аналогичное утверждение справедливо и для нижней границы спектра $E=-2$. Для матриц Вигнера это впервые было доказано Сошниковым [62] на основе работы Синая и Сошникова [63]. Новые доказательства этого результата были недавно получены Тао и Ву [64] и Эрдёшем, Яу и Ином [7] (см. п. 1.6.6). Отметим наличие другого увеличительного множителя $N^{2 / 3}$, который отражает то, что вблизи границ типичное расстояние между собственными значениями равно $N^{-2 / 3}$. Интуитивно ясно, что такое расстояние согласуется с полукруговым законом, так как

$$
\#\left\{\lambda_{j} \geqslant 2-\varepsilon\right\} \approx \frac{N}{2 \pi} \int_{2-\varepsilon}^{2} \sqrt{4-x^{2}} \mathrm{~d} x=\frac{2}{3 \pi} \varepsilon^{3 / 2} N,
$$

так что мы можем ожидать конечное число собственных значений на расстоянии $\varepsilon \sim N^{-2 / 3}$ от границы. Заметим тем не менее, что это рассуждение не 
является строгим, так как для выполнения полукругового закона (1.20) требуется, чтобы тестовый отрезок $[a, b]$ был фиксирован и не зависел от $N$. Недавно в работе [7] нами был доказан локальный полукруговой закон в сильном виде (см. далее теорему 2.19), что дает строгое обоснование предыдущего рассуждения.

Наибольшее собственное значение $\lambda_{N}$ может превышать 2 , но не более чем на величину порядка $O\left(N^{-2 / 3}\right)$. Точнее, функция распределения наибольшего собственного значения задается еще одной универсальной функцией - pacnpeделением Трейси-Уидома [65]:

$$
\lim _{N \rightarrow \infty} \mathrm{P}\left(\lambda_{N} \leqslant 2+\frac{s}{N^{2 / 3}}\right)=F_{2,1}(s):=\exp \left(-\int_{s}^{\infty}(x-s) q^{2}(x) \mathrm{d} x\right),
$$

где $q(s)$ есть решение дифференциального уравнения Пенлеве II $q^{\prime \prime}(s)=s q(s)+$ $2 q^{3}(s)$ с асимптотикой $q(s) \sim \operatorname{Ai}(s)$ при $s=+\infty$ в качестве граничного условия. Можно доказать, что

$$
F_{2,1}(s) \sim 1-\frac{1}{16 \pi s^{3 / 2}} e^{-4 s^{3 / 2} / 3}
$$

при $s \rightarrow \infty$, т. е. собственные значения вне масштаба величин порядка $O\left(N^{-2 / 3}\right)$ субэкспоненциально подавляются. Аналогичная формула имеет место и для симметрических матриц [66]. Заметим, в частности, что этот результат в точности определяет предельное распределение нормы большой матрицы Вигнера.

Универсальность вблизи границ, как правило, исследуется при помощи метода моментов, описанного в п. 1.4. Поправочный член в (1.22) ухудшается с увеличением $k$, но, используя более точную классификацию и оценку возможных структур спаривания, можно определить моменты с точностью до порядка $k=O\left(N^{2 / 3}\right)$, см. [62]. Мы упомянем лишь наиболее простой результат:

$$
\frac{1}{N} \mathrm{E} \operatorname{Tr} H^{2 k}=\frac{2^{2 k}}{\sqrt{\pi k^{3}}}(1+o(1))
$$

при $k=o\left(N^{2 / 3}\right)$. Такой точности достаточно для определения верхней границы спектра матрицы $H$ с точностью почти $N^{-2 / 3}$, так как

$$
\mathrm{P}\left(\lambda_{N} \geqslant 2+\varepsilon\right) \leqslant \frac{\mathrm{E} \operatorname{Tr} H^{2 k}}{(2+\varepsilon)^{2 k}} \leqslant \frac{C N}{k^{3 / 2}(1+\varepsilon / 2)^{2 k}}=o(1)
$$

при $\varepsilon \geqslant(\log N) / k \gg N^{-2 / 3} \log N$. Вычисление (1.42) можно уточнить, включив степени порядка $k \sim N^{2 / 3}$, что приводит к точному определению совместного распределения наибольших собственных значений [62]. Заметим, что в исходной работе Сошникова предполагалось, что распределение одного матричного элемента симметрично, а все его моменты конечны; позднее это условие было ослаблено [67]-[69].

Метод моментов, по-видимому, неприменим за пределами масштаба Сошникова, т. е. при $k$ намного большем, чем $N^{2 / 3}$. С другой стороны, универсальность внутри спектра требует вычисления моментов вплоть до $k \sim O(N)$, так как $1 / k$ - это по существу разрешение, при котором знание точных значений моментов порядка $k$ все еще дает некоторую информацию. Таким образом, 
доказательство универсальности внутри спектра требует совершенно новых методов.

Мы упомянем одно практическое правило. Имеется сильная взаимосвязь между контролем над $e^{-i t H}, H^{k}$ и $(H-z)^{-1}$. По модулю некоторых технических подробностей и логарифмических множителей, следующие три утверждения являются эквивалентными для всех $0<\varepsilon \ll 1$ :

$$
\begin{gathered}
e^{-i t H} \text { контролируемо вплоть до времен }|t| \leqslant \varepsilon^{-1}, \\
H^{k} \text { контролируемо вплоть до степеней } k \leqslant \varepsilon^{-1}, \\
(H-z)^{-1} \text { контролируемо вплоть до значений } \operatorname{Im} z=\eta \geqslant \varepsilon .
\end{gathered}
$$

Связь этих утверждений вытекает из стандартных тождеств

$$
\begin{gathered}
\frac{1}{H-z}=i \int_{0}^{\infty} e^{-i t(H-z)} \mathrm{d} t, \quad z=E+i \eta, \quad \eta>0 \\
e^{-i t H}=\sum_{k=0}^{\infty} \frac{(-i t H)^{k}}{k !}=\frac{1}{2 \pi i} \int_{\gamma} \frac{e^{-i t z}}{H-z} \mathrm{~d} z
\end{gathered}
$$

(где контур $\gamma$ включает в себя весь спектр матрицы $H$ ).

1.5.3. Инвариантные ансамбли. Для ансамблей, которые остаются инвариантными под действием преобразований $H \rightarrow U^{*} H U$ для любой унитарной матрицы $U$ (или, в случае симметрических матриц $H$, для любой ортогональной матрицы $U$ ), совместная функция плотности всех $N$ собственных значений может быть вычислена явно. Такие ансамбли, как правило, задаются плотностью вероятности (1.30). Собственные значения здесь сильно коррелированы и распределены в соответствии с мерой Гиббса с логарифмическим потенциалом взаимодействия на больших расстояниях (эта связь была впервые использована в [70]). Совместная плотность вероятности собственных значений матрицы $H$ может быть вычислена явно:

$$
p_{N}\left(\lambda_{1}, \ldots, \lambda_{N}\right)=\mathrm{const} \prod_{i<j}\left(\lambda_{i}-\lambda_{j}\right)^{\beta} \prod_{j=1}^{N} \exp \left(-N \sum_{j=1}^{N} V\left(\lambda_{j}\right)\right),
$$

где $\beta=1$ для симметрического и $\beta=2$ для эрмитова ансамбля. В частности, в гауссовом случае функция $V$ квадратична, и поэтому совместное распределение собственных значений ГОА $(\beta=1)$ и ГУА $(\beta=2)$ задается формулой

$$
p_{N}\left(\lambda_{1}, \ldots, \lambda_{N}\right)=\mathrm{const} \prod_{i<j}\left(\lambda_{i}-\lambda_{j}\right)^{\beta} \prod_{j=1}^{N} \exp \left(-\frac{1}{4} \beta N \sum_{j=1}^{N} \lambda_{j}^{2}\right) .
$$

Часто бывает полезным представлять эту меру как меру Гиббса в виде

$$
\mu_{N}(\mathrm{~d} \boldsymbol{\lambda})=p_{N}(\boldsymbol{\lambda}) \mathrm{d} \boldsymbol{\lambda}=\frac{e^{-N \mathscr{H}(\boldsymbol{\lambda})}}{Z}, \quad \mathscr{H}(\boldsymbol{\lambda}):=\sum_{i=1}^{N} V\left(\lambda_{i}\right)-\frac{\beta}{N} \sum_{i<j} \log \left|\lambda_{j}-\lambda_{i}\right|
$$

с ограничивающим потенциалом $V(\lambda)=\beta \lambda^{2} / 4$. Доказательство формулы (1.43) получается прямым (но сложным) вычислением; оно основано на подходящем 
выборе замены переменных. Мы дадим набросок лишь для эрмитова (унитарно инвариантного) случая. Ключевым наблюдением является то, что инвариантность меры относительно сопряжений приводит к тому, что собственные значения, организованные в диагональную матрицу $D=\operatorname{diag}\left(\lambda_{1}, \ldots, \lambda_{N}\right)$, являются независимыми от собственных векторов, организованных в унитарную матрицу $U$. Записывая $H=U D U^{*}$, мы получаем, что $\mathscr{P}(H) \mathrm{d} H$ раскладывается следующим образом:

$$
\mathscr{P}(H) \mathrm{d} H=e^{-N \operatorname{Tr} V(H)} \mathrm{d} H=\left[p_{N}\left(\lambda_{1}, \ldots, \lambda_{N}\right) \mathrm{d} \lambda_{1} \cdots \mathrm{d} \lambda_{N}\right] \mathrm{d} U,
$$

где $\mathrm{d} U$ обозначает равномерную меру (Хаара) на унитарной группе $U(N)$, a $p_{N}$ - индуцированная функция плотности на диагональных матрицах (или их элементах). Таким образом, вычисление функции $p_{N}$ сводится к вычислению якобиана замены переменных от элементов матрицы $H$ к координатам параметризации в терминах собственных значений и собственных векторов. В качестве результата получаем

$$
\mathrm{d} H=\text { const }\left[\Delta_{N}(\boldsymbol{\lambda})\right]^{\beta} \mathrm{d} \boldsymbol{\lambda} \mathrm{d} U, \quad \Delta_{N}(\boldsymbol{\lambda}):=\prod_{i<j}\left(\lambda_{i}-\lambda_{j}\right),
$$

где $\beta=1$ в симметрическом и $\beta=2$ в эрмитовом случае, подробности см. в [3] или в разделах 3.1-3.3 книги [1].

Особо стоит отметить появление определителя Вандермонда в (1.43), который непосредственно происходит из интегрирования меры Хаара. Заметим, что тип симметрии ансамбля проявляется в виде показателя $\beta$. Лишь значения $\beta=1,2$ или 4 соответствуют матричным ансамблям вида (1.30), а именно, симметрическим, эрмитовым и кватернионным самодвойственным матрицам. Мы не будем приводить точное определение последнего класса (см., например, [1; гл. 7] или [6]); скажем лишь, что он представляет собой естественное обобщение симметрических или эрмитовых матриц на матрицы с кватернионными элементами, и получаемые матрицы имеют вещественные собственные значения.

В независимости от подлежащего матричного ансамбля, можно тем не менее изучать распределение (1.43) для любого $\beta>0$; такие распределения называются общими $\beta$-ансамблями. В частности, в гауссовом случае $V(\lambda)=$ $\lambda^{2} / 2$ имеются соответствующие ансамбли трехдиагональных матриц для любого $\beta>0$, получаемые последовательным применением преобразований Хаусхолдера; распределения их собственных значений описываются формулой (1.43), см. обзор в [71]. Используя трехдиагональную структуру, можно применять методы теории якобиевых матриц. Например, универсальность для граничных собственных значений можно понимать в том смысле, что они, как показано, сходятся к наименьшим собственным значениям одномерного оператора Шрёдингера при наличии белого шума. В частности, в [72], [73] был определен $\beta$-аналог распределения Трейси-Уидома, следуя гипотезам из [74]. Другой метод, представление броуновской карусели [75], был применен для обобщения закона распределения хвостов промежутков между большими собственными значениями ("оценки Вигнера") на гауссовы $\beta$-ансамбли [76]. Более точно, было показано, что при распределении (1.43) с $V(\lambda)=\lambda^{2} / 2$ вероятность того, что ни одна точка не попадает в фиксированный интервал длины $s$ 
(после локальной перенормировки спектра, при которой типичное расстояние становится равным $2 \pi)$, задается формулой

$q_{s}=\left(\kappa_{\beta}+o(1)\right) s^{\gamma_{\beta}} \exp \left(-\frac{\beta}{64} s^{2}+\left(\frac{\beta}{8}-\frac{1}{4}\right) s\right), \quad \gamma_{\beta}:=\frac{1}{4}\left(\frac{\beta}{2}+\frac{2}{\beta}-3\right), \quad \kappa_{\beta}>0$,

при $s \rightarrow \infty$ (после перехода к пределу при $N \rightarrow \infty$ ).

Свойство универсальности внутри спектра для общих $\beta$-ансамблей, т. е. аналог поведения синус-ядра (1.35), не доказано даже для гауссова случая. Основная трудность заключается в том, что плотность (1.43) задает $N$-частичную систему с взаимодействием на больших расстояниях. Мы можем записать совместную плотность как меру Гиббса (1.45). Тогда мы имеем $N$ частиц с ограничивающим потенциалом $V$; при этом частицы отталкиваются с потенциалом, задающим локально логарифмическое отталкивание, но также имеющим большую (и даже увеличивающуюся) компоненту на больших расстояниях. Стандартные методы, применяемые в статистической физике для построения и анализа мер Гиббса, в этой ситуации, по-видимому, неприменимы. Несмотря на то, что здесь мы не пытаемся построить аналог меры Гиббса с бесконечным объемом, а только хотим подсчитать корреляционные функции, даже это представляется весьма трудной задачей для стандартных методов, если не удастся найти какую-нибудь дополнительную структуру.

1.5.4. Универсальность классических инвариантных ансамблей и ортогональные многочлены. Значительно больше известно о классических инвариантных ансамблях, соответствующих значениям $\beta=1,2,4$, с потенциалом $V$ общего вида. Для этих выделенных значений возникает дополнительная математическая структура, а именно ортогональные многочлены с весовой функцией $e^{-N V(\lambda)}$ на вещественной прямой. Этот подход впервые применили Мета и Годен [1], [77] для вычисления распределения промежутков в гауссовом случае, используя классические ортогональные многочлены Эрмита. Дайсон [10] вычислил локальные корреляционные функции для схожего ансамбля (кругового ансамбля), затем это вычисление было обобщено Метой [11] на стандартные гауссовы ансамбли. Позднее был выработан общий метод, использующий ортогональные многочлены и применимый для класса унитарных ансамблей весьма общего вида (см., например, [1], [2], [78]-[82], где имеются дальнейшие ссылки).

Для простой иллюстрации этой взаимосвязи рассмотрим эрмитов случай $\beta=2$ с гауссовым потенциалом $V(\lambda)=\lambda^{2} / 2$ (который, в силу леммы 1.2 , соответствует ансамблю матриц Вигнера, а именно ГУА). Чтобы еще более упростить изложение, мы перенормируем (лишь в этом рассуждении) собственные значения: $\lambda \rightarrow \sqrt{N} \lambda$, что приводит к исчезновению множителя $N$ из экспоненты в формуле (1.43). (Такая перенормировка работает лишь в гауссовом случае; это лишь техническое средство для упрощения формул.)

Пусть $P_{k}(x)-k$-й ортогональный многочлен с весом $e^{-x^{2} / 2}$ и старшим коэффициентом 1. Рассмотрим соответствующие ортонормированные функции

$$
\psi_{k}(x):=\frac{e^{-x^{2} / 4} P_{k}(x)}{\left\|e^{-x^{2} / 4} P_{k}\right\|},
$$


T. e.

$$
\int \psi_{k}(x) \psi_{\ell}(x) \mathrm{d} x=\delta_{k, \ell} .
$$

В частном случае гауссовой весовой функции многочлены $P_{k}$ являются многочленами Эрмита:

$$
P_{k}(x)=H_{k}(x):=(-1)^{k} e^{x^{2} / 2} \frac{\mathrm{d}^{k}}{\mathrm{~d} x^{k}} e^{-x^{2} / 2},
$$

и

$$
\psi_{k}(x)=\frac{P_{k}(x)}{(2 \pi)^{1 / 4}(k !)^{1 / 2}} e^{-x^{2} / 4},
$$

хотя эти явные формулы не потребуются для дальнейших выводов.

Ключевое наблюдение заключается в том, что в силу простых свойств определителя Вандермонда мы имеем

$$
\Delta_{N}(\mathbf{x})=\prod_{1 \leqslant i<j \leqslant N}\left(x_{j}-x_{i}\right)=\operatorname{det}\left(x_{i}^{j-1}\right)_{i, j=1}^{N}=\operatorname{det}\left(P_{j-1}\left(x_{i}\right)\right)_{i, j=1}^{N},
$$

где мы воспользовались тем, что $P_{j}(x)=x^{j}+\cdots-$ многочлен степени $j$ со старшим коэффициентом 1 . Введем ядро

$$
K_{N}(x, y):=\sum_{k=0}^{N-1} \psi_{k}(x) \psi_{k}(y)
$$

т. е. ядро, соответствующее проекции на подпространство, порожденное первыми $K$ ортогональными функциями. Тогда из (1.48) непосредственно получаем

$$
\begin{aligned}
p_{N}\left(x_{1}, \ldots, x_{N}\right) & =C_{N}\left[\operatorname{det}\left(P_{j-1}\left(x_{i}\right)\right)_{i, j=1}^{N}\right]^{2} \prod_{i=1}^{N} e^{-x_{i}^{2} / 2} \\
& =C_{N}^{\prime}\left[\operatorname{det}\left(\psi_{j-1}\left(x_{i}\right)\right)_{i, j=1}^{N}\right]^{2}=C_{N}^{\prime} \operatorname{det}\left(K_{N}\left(x_{i}, x_{j}\right)\right)_{i, j=1}^{N}
\end{aligned}
$$

(на последнем шаге мы использовали то, что квадрат матрицы $\left(\psi_{j-1}\left(x_{i}\right)\right)_{i, j=1}^{N}$ есть в точности $\left.\left(K_{N}\left(x_{i}, x_{j}\right)\right)_{i, j=1}^{N}\right)$. Для простоты мы не выписывали константы в явном виде.

Для вычисления корреляционных функций разложим определитель следующим образом:

$$
\begin{aligned}
p_{N}^{(k)}\left(x_{1}, \ldots, x_{k}\right) & =C_{k, N} \int_{\mathbb{R}^{N-k}} \operatorname{det}\left(K_{N}\left(x_{i}, x_{j}\right)\right)_{i, j=1}^{N} \prod_{i=k+1}^{N} \mathrm{~d} x_{i} \\
& =C_{k, N} \sum_{\sigma, \tau \in S_{N}}(-1)^{\tau+\sigma} \int_{\mathbb{R}^{N-k}} \prod_{j=1}^{N} \psi_{\sigma(j)-1}\left(x_{j}\right) \psi_{\tau(j)-1}\left(x_{j}\right) \prod_{i=k+1}^{N} \mathrm{~d} x_{i} \\
& =C_{k, N} \sum_{\alpha_{1}<\cdots<\alpha_{k}} \sum_{\sigma, \tau \in S_{k}}(-1)^{\tau+\sigma} \prod_{j=1}^{k} \psi_{\alpha_{\sigma(j)}-1}\left(x_{j}\right) \psi_{\alpha_{\tau(j)}-1}\left(x_{j}\right) \\
& =C_{k, N} \sum_{\alpha_{1}<\cdots<\alpha_{k}}\left[\operatorname{det}\left(\psi_{\alpha_{j}-1}\left(x_{j}\right)\right)_{i, j=1}^{k}\right]^{2},
\end{aligned}
$$

4 УМН, т. 66, вып. 3 
где $S_{N}$ обозначает группу перестановок $N$ элементов, а $(-1)^{\tau}$ обозначает четность перестановки. В третьей строке мы воспользовались соотношением (1.47) для вычисления интеграла, положив $\sigma(j)=\tau(j)$ для всех $j \geqslant k+1$ и обозначив через $\left\{\alpha_{1}, \ldots, \alpha_{k}\right\}$ порядок на множестве $\{\sigma(1), \ldots, \sigma(k)\}=\{\tau(1), \ldots, \tau(k)\}$. Наконец, используя тот факт, что матрица $\left[K_{N}\left(x_{i}, x_{j}\right)\right]_{i, j=1}^{k}$ может быть представлена в виде $A^{t} A$ с $A_{i j}=\psi_{i-1}\left(x_{j}\right)$, а также формулу разложения КошиБине для определителя произведения матриц, мы получаем

$$
\operatorname{det}\left[K_{N}\left(x_{i}, x_{j}\right)\right]_{i, j=1}^{k}=\sum_{\alpha_{1}<\cdots<\alpha_{k}}\left[\operatorname{det}\left(\psi_{\alpha_{j}-1}\left(x_{j}\right)\right)_{i, j=1}^{k}\right]^{2} .
$$

По модулю константы, которую можно явно вычислить, мы тем самым доказали, что

$$
p_{N}^{(k)}\left(x_{1}, \ldots, x_{k}\right)=\frac{(N-k) !}{N !} \operatorname{det}\left[K_{N}\left(x_{i}, x_{j}\right)\right]_{i, j=1}^{k},
$$

т. е. корреляционные функции имеют структуру определителя.

Для того чтобы увидеть, как появляется синус-ядро (1.33), нам понадобится фундаментальное алгебраическое свойство ортогональных многочленов - формула Кристоффеля-Дарбу:

$$
K_{N}(x, y)=\sum_{j=0}^{N-1} \psi_{j}(x) \psi_{j}(y)=\sqrt{N}\left[\frac{\psi_{N}(x) \psi_{N-1}(y)-\psi_{N}(y) \psi_{N-1}(x)}{x-y}\right] .
$$

Более того, ортогональные многочлены больших степеней имеют следующее асимптотическое поведение при $N \rightarrow \infty$ :

$$
\begin{aligned}
\psi_{2 m}(x) & \approx \frac{(-1)^{m}}{N^{1 / 4} \sqrt{\pi}} \cos (\sqrt{N} x)+o\left(N^{-1 / 4}\right), \\
\psi_{2 m+1}(x) & \approx \frac{(-1)^{m}}{N^{1 / 4} \sqrt{\pi}} \sin (\sqrt{N} x)+o\left(N^{-1 / 4}\right)
\end{aligned}
$$

для любого $m$, удовлетворяющего условию $|2 m-N| \leqslant C$. Это приближение равномерно при $|x| \leqslant C N^{-1 / 2}$. Данные формулы будут полезны при подстановке значения $E=0$ в (1.35). Так как мы отнормировали собственные значения на множитель $\sqrt{N}$, соотношения между переменными из $(1.35)$ (для $k=2$ ) и переменными $x, y$ имеют вид

$$
x=\sqrt{N}\left(E+\frac{\alpha_{1}}{N \varrho(E)}\right), \quad y=\sqrt{N}\left(E+\frac{\alpha_{2}}{N \varrho(E)}\right) .
$$

При других значениях энергии $E$ требуются несколько иные асимптотические формулы для ортогональных многочленов. Таким образом, мы имеем

$$
K_{N}(x, y) \approx \frac{1}{\pi} \frac{\sin (\sqrt{N} x) \cos (\sqrt{N} y)-\sin (\sqrt{N} y) \cos (\sqrt{N} x)}{x-y}=\frac{\sin \sqrt{N}(x-y)}{\pi(x-y)} .
$$

Используя соотношения (1.50) и тот факт, что $\varrho(0)=\pi^{-1}$, мы получаем

$$
\frac{1}{\varrho(0) \sqrt{N}} K_{N}(x, y) \approx \frac{\sin \pi\left(\alpha_{1}-\alpha_{2}\right)}{\pi\left(\alpha_{1}-\alpha_{2}\right)},
$$


что дает в точности формулу (1.35) при $E=0$ после избавления от перенормировки $\lambda \rightarrow \sqrt{N} \lambda$.

Основным техническим средством в этом рассуждении являлись асимптотические формулы (1.49) для ортогональных многочленов. В случае классических ортогональных многочленов (возникающих в стандартных ансамблях Вигнера и Уишарта) такие формулы обычно получаются при помощи асимптотик Лапласа из их целочисленных представлений. Для потенциала $V$ общего вида соответствующий анализ становится достаточно сложным и зависит от свойств регулярности функции $V$. Один из успешных подходов в этом направлении был реализован Фокасом, Итсом и Китаевым [81], а также П. Дефтом и его коллегами при помощи метода Римана-Гильберта (см. [2], где имеются дополнительные ссылки). Альтернативный подход с использованием более прямых методов с ортогональными многочленами был предложен в [83], [84].

В этой весьма активной области исследований, связанных с инвариантными ансамблями, постоянно появляются улучшения и уточнения существующих результатов, открывающие новые взаимосвязи между случайными матрицами, ортогональными многочленами, комплексным анализом и даже комбинаторикой (см. [2]). Тем не менее, общим средством всегда является явная формула (1.43) совместной плотности вероятности, позволяющая привлекать ортогональные многочлены. Далее мы перейдем от этого раздела к рассмотрению ансамблей, для которых такая явная формула отсутствует; наиболее известным из них являются матрицы Вигнера. За исключением гауссова случая, здесь отсутствуют явные формулы для совместного распределения собственных значений. Таким образом, фундаментальная алгебраическая взаимосвязь между ансамблями собственных значений и ортогональными многочленами не может быть использована, и необходимо разрабатывать совершенно новые методы. В следующем разделе мы дадим обзор последних результатов в этом направлении.

\section{6. Локальные статистики собственных значений: новые резуль- таты.}

1.6.1. Эрмитовы матрицы с гауссовыми конволюциями. Первый строгий частичный результат об универсальности внутри спектра в неунитарном случае был получен Йоханссоном [13]; см. также работу Бен Аруса и Пеше [85], где результат [13] обобщается на всю внутренность спектра, и недавно полученное улучшение [86], ослабляющее условие на моменты. Основной результат утверждает, что свойство универсальности внутри спектра имеет место для гауссово делимых эрмитовых ансамблей, т. е. эрмитовых ансамблей вида

$$
H=\sqrt{1-\varepsilon} \widehat{H}+\sqrt{\varepsilon} V
$$

где $\widehat{H}$ - эрмитова матрица Вигнера, $V$ - независимая стандартная матрица ГУА, а $\varepsilon$ - положительная константа порядка один, независимая от $N$.

Мы будем обычно использовать параметризацию

$$
H=e^{-t / 2} \widehat{H}+\left(1-e^{-t}\right)^{1 / 2} V .
$$

Будучи вложенной в поток, переменная $t$ может быть интерпретирована как время в прочессе Орнштейна-Уленбека. Этот формализм выражает идею 
о том, что матрицы с гауссовыми конволюциями могут быть получены как матричнозначный стохастический процесс, а именно как решение следующего стохастического дифференциального уравнения:

$$
\mathrm{d} H_{t}=\frac{1}{\sqrt{N}} \mathrm{~d} \beta_{t}-\frac{1}{2} H_{t} \mathrm{~d} t, \quad H_{0}=\widehat{H},
$$

где $\beta_{t}$ - эрмитов матричнозначный процесс, диагональные элементы которого - стандартные вещественные броуновские движения, а элементы вне диагонали - стандартные комплексные броуновские движения. Распределение вероятностей для решения уравнения (1.54) при любом фиксированном $t$ совпадает с распределением (1.53). Заметим, что бесконечное время $t=\infty$ соответствует ансамблю ГУА, т. е. матрицы (1.53) являются интерполяиией между матрицей Вигнера $\widehat{H}$ и ГУА. Такая точка зрения будет чрезвычайно полезной в дальнейшем, так как она позволяет сравнивать матрицы Вигнера с гауссовыми матрицами, при условии, что имеется контроль над развитием времени.

Наряду с этим, можно рассматривать эволюцию функции плотности $u_{t}$ вещественных и мнимых частей матричных элементов при помощи образующеи процесса Орнштейна-Уленбека

$$
\partial_{t} u_{t}=A u_{t}, \quad A:=\frac{1}{4} \frac{\partial^{2}}{\partial x^{2}}-\frac{x}{2} \frac{\partial}{\partial x},
$$

где начальное условие $u_{0}(x)$ является плотностью (по отношению к обратимой гауссовой мере) распределения вещественных и мнимых частей элементов матрицы $\sqrt{N} \widehat{H}$. Для диагональных элементов используется процесс Орнштейна-Уленбека с несколько иной нормализацией. Процесс Орнштейна-Уленбека (1.55) сохраняет нулевое математическое ожидание и дисперсию $1 / 2$, если начальное условие $u_{0}$ имеет эти значения.

Совместное распределение собственных значений гауссово делимых эрмитовых случайных матриц вида (1.53) по-прежнему имеет некоторую структуру определителя. Формулы становятся несколько проще, если мы запишем

$$
H=\sqrt{1-\varepsilon}(\widehat{H}+a V)
$$

где $a=\sqrt{\varepsilon /(1-\varepsilon)}$, т. е. используем стандартную гауссову конволюцию

$$
\widetilde{H}=\widehat{H}+a V
$$

и затем в конце сделаем перенормировку. Заметим, что соотношение (1.56) можно получить, дав матричным элементам проэволюционировать при помощи стандартных броуновских движений $\beta$ до времени $t=a^{2}$, т. е. решив уравнение

$$
\mathrm{d} \widetilde{H}_{t}=\frac{1}{\sqrt{N}} \mathrm{~d} \beta_{t}, \quad \widetilde{H}_{0}=\widehat{H} .
$$

Более того, для сохранения условий нормировки из [14], которые согласуются с условиями из [13], мы предположим, что элементы матрицы Вигнера $\widehat{H}$ и матрицы ГУА $V$ имеют дисперсию $1 /(4 N)$, а не $1 / N$, как указано в определении 1.1 . Это означает, что собственные значения отнормированы на множитель $1 / 2$ по 
сравнению с соглашением из предыдущих пунктов, а полукруговой закон (1.20) преобразован в $2 \pi^{-1} \sqrt{\left(1-x^{2}\right)_{+}}$. Это соглашение применяется лишь до конца этого раздела.

Пусть $\mathbf{y}=\left(y_{1}, \ldots, y_{N}\right)$ обозначает собственные значения матрицы $\widehat{H}, \mathbf{a} \mathbf{x}=$ $\left(x_{1}, \ldots, x_{N}\right)$ - собственные значения матрицы $\widetilde{H}$. Тогда мы имеем следующие формулы представления (эти формулы в несколько измененном виде были приведены и использованы Йоханссоном в [13], мотивацией ему послужили аналогичные формулы Брезина и Хиками [12]).

ЛЕмма 1.4 [14; предложение 3.2]. Пусть $V$ - матрица ГУА. Для любой фиксированной эрмитовой матрицы $\widehat{H}$ с собственными значениями у функиия плотности собственных значении $\mathbf{x}$ матрицы $\widetilde{H}=\widehat{H}+a V$ задается формулой

$$
q_{S}(\mathbf{x} ; \mathbf{y}):=\frac{1}{(2 \pi S)^{N / 2}} \frac{\Delta_{N}(\mathbf{x})}{\Delta_{N}(\mathbf{y})} \operatorname{det}\left(e^{-\left(x_{j}-y_{k}\right)^{2} /(2 S)}\right)_{j, k=1}^{N},
$$

где $S=a^{2} / N$, a $\Delta_{N}$ обозначает определитель Вандермонда (1.48). Далее, m-точечные корреляционные функиии собственных значений матрицы $\widetilde{H}=$ $\widehat{H}+a V$,

$$
p_{N, \mathbf{y}}^{(m)}\left(x_{1}, \ldots, x_{m}\right):=\int_{\mathbb{R}^{N-m}} q_{S}\left(x_{1}, \ldots, x_{N} ; \mathbf{y}\right) \mathrm{d} x_{m+1} \cdots \mathrm{d} x_{N},
$$

задаются следующей формулой:

$$
p_{N, \mathbf{y}}^{(m)}\left(x_{1}, \ldots, x_{m}\right)=\frac{(N-m) !}{N !} \operatorname{det}\left(\mathscr{K}_{N}^{S}\left(x_{i}, x_{j} ; \mathbf{y}\right)\right)_{i, j=1}^{m}, \quad S=\frac{a^{2}}{N} .
$$

Здесь ми ввели

$$
\begin{gathered}
\mathscr{K}_{N}^{S}(u, v ; \mathbf{y}):=\frac{1}{(2 \pi i)^{2}(v-u) S} \int_{\gamma} \mathrm{d} z \int_{\Gamma} \mathrm{d} w\left(e^{-(v-u)(w-r) / S}-1\right) \prod_{j=1}^{N} \frac{w-y_{j}}{z-y_{j}} \\
\quad \times \frac{1}{w-r}\left(w-r+z-u-S \sum_{j} \frac{y_{j}-r}{\left(w-y_{j}\right)\left(z-y_{j}\right)}\right) e^{\left(w^{2}-2 u w-z^{2}+2 u z\right) /(2 S)},
\end{gathered}
$$

где $r \in \mathbb{R}$ - произвольная константа. Кривые интегрирования $\gamma$ и Г в комплексной плоскости задаются следующим образом: $\gamma=\gamma_{+} \cup \gamma_{-}-$обгединение двух прямых $\gamma_{+}: \tau \rightarrow-\tau+i \omega u \gamma_{-}: \tau \rightarrow \tau-i \omega(\tau \in \mathbb{R})$ для любого фиксированного $\omega>0, a \Gamma-\tau \rightarrow i \tau(\tau \in \mathbb{R})$.

Заметим, что Г можно сдвинуть до любой вертикальной прямой, так как выражение под интегралом является целой функцией от $w$ и имеет гауссово убывание при $|\operatorname{Im} w| \rightarrow \infty$. Константы $r \in \mathbb{R}$ и $\omega>0$ (появляющиеся в определении контура $\gamma$ ) могут быть произвольными и при оценках контурного интеграла могут быть выбраны произвольно.

Ключевым шагом в доказательстве формулы (1.58) является интеграл Хариш-Чандры-Ициксона-Зубера [87]:

$$
\int_{U(N)} e^{\operatorname{Tr}\left(U A U^{*} B\right)} \mathrm{d} U=\mathrm{const} \frac{\operatorname{det}\left(e^{a_{i} b_{j}}\right)_{i, j=1}^{N}}{\Delta_{N}(\mathbf{a}) \Delta_{N}(\mathbf{b})},
$$


где $A, B$ - две эрмитовы матрицы с собственными значениями $\mathbf{a}=\left(a_{1}, \ldots, a_{N}\right)$ и $\mathbf{b}=\left(b_{1}, \ldots, b_{N}\right)$, а интеграл берется по унитарной группе $U(N)$ с мерой Хаapa. Заметим, что на этом шаге существенно используется унитарная инвариантность (или эрмитов характер матрицы $H$ ); аналогичные простые формулы для других симметрических групп неизвестны, см. [88].

Для любой тестовой функции $f$ и для любой фиксированной матрицы $\widehat{H}$ мы имеем

$$
\int f(\mathbf{x}) q_{S}(\mathbf{x} ; \mathbf{y}) \mathrm{d} \mathbf{x}=\text { const } \int f(\mathbf{x}) e^{-N \operatorname{Tr}(\widetilde{H}-\widehat{H})^{2} / 2} \mathrm{~d} \widetilde{H},
$$

где мы использовали тот факт, что $V=a^{-1}(\widetilde{H}-\widehat{H})$ является ГУА-матрицей с распределением

$$
\mathscr{P}(V) \mathrm{d} V=e^{-N \operatorname{Tr} V^{2} / 2} \mathrm{~d} V .
$$

Приведем матрицу $\widetilde{H}$ к диагональному виду: $\widetilde{H}=U X U^{*}$, где $X=\operatorname{diag}(\mathbf{x})$. Тогда, используя (1.46), получаем

$$
\begin{aligned}
\int f(\mathbf{x}) q_{S}(\mathbf{x} ; \mathbf{y}) \mathrm{d} \mathbf{x} \\
=\operatorname{const} \int_{\mathbb{R}^{N}} \int_{U(N)} f(\mathbf{x}) \exp \left(-\frac{1}{2 a^{2}} N \operatorname{Tr}\left(U X U^{*}-\widehat{H}\right)^{2}\right) \mathrm{d} U_{\Delta_{N}^{2}(\mathbf{x}) \mathrm{d} \mathbf{x}} \\
=\operatorname{const} \int_{\mathbb{R}^{N}}\left[\int_{U(N)} \exp \left(\frac{N}{a^{2}} \operatorname{Tr} U X U^{*} \widehat{H}\right) \mathrm{d} U\right] \\
\quad \times f(\mathbf{x}) \exp \left(-\frac{N}{2 S} \sum_{i}\left(x_{i}^{2}+y_{i}^{2}\right)\right) \Delta_{N}^{2}(\mathbf{x}) \mathrm{d} \mathbf{x} \\
=\operatorname{const} \int_{\mathbb{R}^{N}} f(\mathbf{x}) \frac{\operatorname{det}\left(e^{x_{i} y_{j} / S}\right)_{i, j=1}^{N}}{\Delta_{N}(\mathbf{x}) \Delta_{N}(\mathbf{y})} \exp \left(-\frac{N}{2 S} \sum_{i}\left(x_{i}^{2}+y_{i}^{2}\right)\right) \Delta_{N}^{2}(\mathbf{x}) \mathrm{d} \mathbf{x} \\
=\operatorname{const} \int_{\mathbb{R}^{N}} f(\mathbf{x}) \frac{\Delta_{N}(\mathbf{x})}{\Delta_{N}(\mathbf{y})} \operatorname{det}\left(\exp \left(-\frac{1}{2 S}\left(x_{i}-y_{j}\right)^{2}\right)\right)_{i, j=1}^{N} \mathrm{~d} \mathbf{x},
\end{aligned}
$$

что доказывает формулу (1.58) (по модулю константы). Это вычисление показывает, каким образом в гауссовых конволюциях возникает структура определителя Вандермонда. Доказательство контурного интегрального представления (1.60) из (1.58) несколько более сложно, см. подробности в [14; предложение 3.2] (или в [13; предложение 2.3]).

В случае, когда имеется представление (1.60), ключевая идея заключается в том, чтобы рассматривать его как комплексный интеграл, для которого можно изучать асимптотики Лапласа и производить вычисления с седловыми точками. Более точно, путем некоторых прямых алгебраических вычислений его можно привести к следующему виду (см. [14; раздел 3.1]), где мы уже сделали замену переменных в аргументе, чтобы выявить микроскопическую структуру. Для любого фиксированного $|u|<1$ и $t=a^{2}$ мы находим из (1.60), что

$$
\frac{1}{N \varrho(u)} \mathscr{K}_{N}^{t / N}\left(u, u+\frac{\tau}{N \varrho(u)} ; \mathbf{y}\right)=N \int_{\gamma} \frac{\mathrm{d} z}{2 \pi i} \int_{\Gamma} \frac{\mathrm{d} w}{2 \pi i} h_{N}(w) g_{N}(z, w) e^{N\left(f_{N}(w)-f_{N}(z)\right)},
$$


где

$$
\begin{aligned}
f_{N}(z) & :=\frac{1}{2 t}\left(z^{2}-2 u z\right)+\frac{1}{N} \sum_{j} \log \left(z-y_{j}\right) \\
g_{N}(z, w) & :=\frac{1}{t(w-r)}[w-r+z-u]-\frac{1}{N(w-r)} \sum_{j} \frac{y_{j}-r}{\left(w-y_{j}\right)\left(z-y_{j}\right)}, \\
h_{N}(w) & :=\frac{1}{\tau}\left(e^{-\tau(w-r) /(t \varrho(u))}-1\right) .
\end{aligned}
$$

Обратим внимание на множитель $N$ в показателе экспоненты в $(1.61)$, который указывает на то, что главный вклад в интеграл дают седловые точки, т.е. значения $z$ и $w$, при которых $f_{N}^{\prime}(z)=f_{N}^{\prime}(w)=0$. Заметим, что

$$
f_{N}^{\prime}(z)=\frac{z-u}{t}+\frac{1}{N} \sum_{j} \frac{1}{z-y_{j}},
$$

т. е. производная фактически задается эмпирическим преобразованием Стилтьеса (1.23) собственных значений матрицы $\widehat{H}$. Предположим, что имеет место полукруговой закон Вигнера, т. е. соотношение (1.28); тогда седловые точки $z_{N}$, в которых $f_{N}^{\prime}\left(z_{N}\right)=0$, могут быть хорошо аппроксимированы решением уравнения

$$
\frac{z-u}{t}+2\left(z-\sqrt{z^{2}-1}\right)=0
$$

(формула для преобразования Стилтьеса несколько отличается от (1.28) ввиду другой нормировки матрицы $\widehat{H})$. Легко видеть, что имеется два решения $z^{ \pm}$, мнимые части которых имеют вид $\pm 2 t i \sqrt{1-u^{2}}+O\left(t^{2}\right)$ при малых $t$.

Как только найдены седловые точки, контуры интегрирования $\gamma$ и Г в (1.61) можно сдвинуть так, что они проходили через седловые точки, подходя к ним таким образом, что значение $f_{N}$ вещественно, а его вторая производная имеет "хороший знак", так что применима обычная аппроксимация в седловых точках. Всего имеется четыре пары седел $\left(z_{N}^{ \pm}, w_{N}^{ \pm}\right)$, но только две из них дают вклад в члены высшего порядка. Явное вычисление дает синус-ядро (1.33) для $\mathscr{K}_{N}$ в пределе при $N \rightarrow \infty$.

Основным техническим требованием является обоснование возможности аппроксимации при помощи полукруга, использованной при переходе от (1.62) к (1.63) вблизи седловой точки (некоторые оценки необходимы также и вдали от седловых точек, но их, как правило, легче получить). Стандартное рассуждение для полукругового закона, приведенное в п. 1.4.2, проходит только для любого фиксированного $z \mathrm{c} \operatorname{Im} z>0$, независимо от $N$; здесь особенно важно, что неравенство $\operatorname{Im} z>0$ выполнено равномерно по $N$. Таким образом, это рассуждение можно использовать для обоснования перехода к (1.63) только при фиксированном $t>0$. Напомним, что $t=a^{2}$ есть дисперсия гауссовой конволюции. Этот подход был в сущности использован Йоханссоном в доказательстве [13] того, что универсальность синус-ядра (1.35) имеет место в случае, если матрица Вигнера содержит гауссову компоненту, сравнимую с ее глобальным размером.

Одно из следствий локального полукругового закона, описанного в п. 1.1.1, заключается в том, что переход от (1.62) к (1.63) может быть обоснован даже 
для очень коротких времен $t$. В сущности, допускается любое время порядка $1 / N$ (с некоторым логарифмическим множителем), но по техническим причинам мы получили оценки лишь для $t=N^{-1+\varepsilon}$ с любым $\varepsilon>0$, а также показали, что контур интегрирования в (1.61) задается синус-ядром даже для таких коротких времен [14]. Это привело к доказательству универсальности синус-ядра в виде (1.35) для любого фиксированного $E$ внутри спектра для эрмитовых матриц с гауссовой компонентой, имеющей дисперсию $N^{-1+\varepsilon}$.

1.6.2. Гауссовы конволюции для произвольных матриц: локальный релаксационный поток. Метод, изложенный в п. 1.6.1, существенно опирается на формулы (1.60) типа Брезина-Хиками, которые существуют лишь для эрмитовых матриц. Для ансамблей с другими симметриями, в частности, для симметрических матриц Вигнера, был необходим новый метод. В серии работ [89], [16], [6]-[9] нами был разработан подход, основанный на идеях из гидродинамики взаимодействующих систем частиц. Более детально этот подход будет изложен в разделе 3, здесь мы лишь наметим основные моменты.

Отправной точкой было следующее ключевое наблюдение Дайсона [15] 1962 г. Для произвольной фиксированной матрицы $\widehat{H}$ рассмотрим решение $\widetilde{H}_{t}$ уравнения (1.57). Напомним, что для любого фиксированного $t$ матрица $\widetilde{H}_{t}$ имеет то же распределение, что и $\widehat{H}+\sqrt{t} V$, где $V$ - стандартная матрица ГУА (независимая от $\widehat{H}$ ). Дайсон заметил, что эволюция собственных значений под действием потока $\widetilde{H}_{t}$ задается спаренной системой стохастических дифференциальных уравнений, обычно называемой броуновским движением Дайсона (или кратко БДД). Для удобства мы заменим броуновские движения процессами Орнштейна-Уленбека, чтобы сохранять дисперсию постоянной, т. е. мы будем использовать уравнение (1.54) вместо (1.57) для порождения матричного потока. Используемое нами броуновское движение Дайсона (которое мы все равно будем называть БДД) задается следующей системой стохастических дифференциальных уравнений на собственные значения $\boldsymbol{\lambda}(t)=$ $\left(\lambda_{1}(t), \ldots, \lambda_{N}(t)\right)$ (см., например, [3; раздел 4.3.1]):

$$
\mathrm{d} \lambda_{i}=\frac{\mathrm{d} B_{i}}{\sqrt{N}}+\left[-\frac{\beta}{4} \lambda_{i}+\frac{\beta}{2 N} \sum_{j \neq i} \frac{1}{\lambda_{i}-\lambda_{j}}\right] \mathrm{d} t, \quad 1 \leqslant i \leqslant N,
$$

где $\left\{B_{i}: 1 \leqslant i \leqslant N\right\}$ - набор независимых броуновских движений. Начальное условие $\boldsymbol{\lambda}(0)$ задается собственными значениями матрицы $\widehat{H}$. Выбор параметра $\beta=2$ соответствует эрмитову случаю, но процесс (1.64) корректно определен для любого $\beta \geqslant 1$, и собственные значения не накладываются друг на друга из-за сильного отталкивания. Порог $\beta=1$ является критическим для отсутствия наложения. При $t \rightarrow \infty$ распределение собственных значений $\boldsymbol{\lambda}(t)$ сходится к распределению гауссова $\beta$-ансамбля (1.44) как глобальная инвариантная мера; например, при $\beta=2$ оно сходится к ГУА.

Используя броуновское движение Дайсона, вопрос об универсальности для гауссово делимых ансамблей можно интерпретировать как вопрос о времени, необходимом для достижения равновесия в БДД. Время, необходимое для достижения глобального равновесия, имеет порядок один, но, как мы в итоге доказали в [7], локальное равновесие достигается гораздо быстрее, а именно за время порядка $t \sim N^{-1}$. Так как локальные статистики собственных значений 
зависят исключительно от локального равновесия, это означает, что локальные статистики гауссово делимых ансамблей с незначительной гауссовой компонентой размера $N^{-1+\varepsilon}$ уже являются универсальными.

Заметим, что, используя соотношение между гауссово делимыми ансамблями и временем релаксации БДД, результат Йоханссона [13] можно интерпретировать как утверждение от том, что локальные статистики ГУА достигаются в БДД за время порядка не более 1. Наш результат из [14], обсуждавшийся в п. 1.6.1, показывает, что локальное равновесие достигается уже за время $t \sim N^{-1+\varepsilon}$. Это, однако, является лишь переформулировкой результатов, так как идеи из гидродинамики не использовались ни в [13], ни в [14]. В частности, эти доказательства проходят лишь для эрмитовых матриц, так как они используют разновидности формулы Брезина-Хиками.

Для того чтобы установить универсальность в полной общности, мы разработали чисто гидродинамический подход, основанный на скорости релаксации БДД. Ключевой особенностью этого подхода является то, что он не накладывает ограничений на тип симметрии; рассуждение проходит одинаково для симметрических, эрмитовых и кватернионных самодвойственных ансамблей; более того, после некоторых видоизменений этот подход работает и для случайных ковариационных матриц.

Нашей первой работой, в которой использовались идеи из гидродинамики, была статья [89]. В ней мы обобщили результат Йоханссона [13] на эрмитовы ансамбли с гауссовой компонентой размера $t \gg N^{-3 / 4}$, основываясь на том, что локальные статистики собственных значений зависят исключительно от приближения к локальному равновесию, которое в общем случае достигается быстрее, чем глобальное равновесие. К сожалению, описание локальных равновесий в [89] все еще использовало явные представления корреляционных функций ортогональными многочленами (следуя, например, [82]), и обобщение на произвольные ансамбли с использованием этого метода было непростым делом (см. [90], где подход [82] обобщен на симметрические матрицы и доказана универсальность на границах спектра).

Для того чтобы отказаться от использования ортогональных многочленов, мы ввели в [16] новые гидродинамические методы, которые позволили полностью исключить явные формулы и дать общее доказательство универсальности для симметрических и эрмитовых матриц Вигнера с малыми гауссовыми конволюциями. Размер гауссовой компоненты или, эквивалентно, время, необходимое для достижения локального равновесия в достаточно сильном смысле, увеличилось с $N^{-3 / 4}$ до $N^{-\xi}$ с малым положительным $\xi$, но метод стал более общим. Данный результат был в дальнейшем обобщен в [6] на кватернионные самодвойственные матрицы Вигнера и выборочные ковариационные матрицы, а также на обобщенные матрицы Вигнера в [9], [8] (см. определение 1.1). Наконец, в [7] нами было показано, что локальное равновесие достигается уже за время $t \geqslant N^{-1+\varepsilon}$, которое фактически является оптимальным. Более важным является то, что гидродинамический метод применим не только для этих конкретных ансамблей, но также дает концептуальное понимание того, что возникновение универсальности вызвано приходом БДД в локальное равновесие. 
Наш гидродинамический подход состоит из двух частей. Во-первых, имеется общая теорема о том, что при некоторых структурных условиях и условиях выпуклости на гамильтониан $\mathscr{H}$ равновесной меры БДД (см. специальный случай в (1.45)) и при наличии сильного контроля над локальной плотностью собственных значений, локальное равновесие достигается за короткое время $t \sim N^{-\xi}$, $\xi>0$, т. е. локальные корреляционные функции, нормированные в виде (1.35), совпадают с теми же корреляционными функциями в равновесии. Согласно общему критерию Бакри-Эмери [91], скорость сходимости к глобальному равновесию для БДД зависит от нижней границы на гессиан гамильтониана $\mathscr{H}$, которая в нашем случае имеет порядок один. Ключевая идея заключается в том, чтобы ускорить эту сходимость путем изменения гамильтониана. Мы добавляем к $\mathscr{H}$ вспомогательный потенциал вида

$$
W(\boldsymbol{\lambda})=\frac{1}{2 R^{2}} \sum_{j}\left(\lambda_{j}-\gamma_{j}\right)^{2},
$$

где $R \ll 1$ - параметр, зависящий от $N$, а $\gamma_{j}$ - классические расположения собственных значений, задаваемые формулой

$$
N \int_{-\infty}^{\gamma_{j}} \varrho(x) \mathrm{d} x=j .
$$

Здесь $\varrho(x)$ - предельная плотность, например, $\varrho(x)=\varrho_{\mathrm{sc}}(x)$ для матриц Вигнера. Гамильтониан $\widehat{\mathscr{H}}:=\mathscr{H}+W$ порождает новый стохастический поток собственных значений, называемый локалъным релаксационным потоком. Равновесная мера Гиббса, задаваемая гамильтонианом $\widehat{\mathscr{H}}$, будет называться $n c е в-$ доравновесной мерой. Сходимость к равновесию для этого потока быстрее: равновесие достигается за время порядка $R^{2}$. На самом деле, ввиду сильного отталкивания собственных значений (которое выражается особым отталкивающим потенциалом (1.64)), сходимость происходит еще быстрее для наблюдаемых, которые зависят только от разностей собственных значений. Затем мы показываем, что модифицированные динамики на самом деле не сильно отличаются от исходных, используя тот факт, что вспомогательный потенциал $W$ имеет малый типичный размер. Более точно, нам будет необходимо показать, что собственные значения $\lambda_{j}$ лежат вблизи $\gamma_{j}$ с точностью $N^{-1 / 2-\varepsilon}$, т. е. для некоторого $\varepsilon>0$ выполнено соотношение

$$
\mathrm{E} \frac{1}{N} \sum_{i=1}^{N}\left(\lambda_{i}-\gamma_{i}\right)^{2}=N^{-1-2 \varepsilon}
$$

Это свойство является ключевым для нашей общей теоремы, и оно будет доказано на основе сильного контроля над локальной плотностью. Показатель $\xi$ в масштабе времени $t \sim N^{-\xi}$ есть в сущности число $2 \varepsilon$, появляющееся в оценке (1.66).

Вторая часть гидродинамического подхода состоит в доказательстве необходимых для общей теоремы свойств локальной плотности, в особенности (1.66). На этом шаге проявляются специфические свойства матричных ансамблей. Для того чтобы показать, что локальное равновесие достигается за время порядка $t \sim N^{-\xi}, \xi>0$, нам необходимо определить расположение собственных 
значений с точностью по крайней мере $N^{-1 / 2-\varepsilon}, \varepsilon=\xi / 2$. Чтобы получить оптимальное время прихода в равновесие $t \gg N^{-1}$, расположение собственных значений необходимо определить фактически с точностью $N^{-1}$, по аналогии с [14]. Грубо говоря, точность нахождения расположения собственных значений соответствует масштабу $\eta$, для которого имеет место локальный полукруговой закон, и это будет основным средством для проверки соотношения (1.66). Техническая трудность заключается в том, что нам необходим достаточно хороший контроль над локальной плотностью также и вблизи границ спектра, так как соотношение (1.66) включает все собственные значения. Несмотря на то, что нас интересует лишь универсальность внутри спектра, т. е. локальное поведение вдали от границ, нам все равно требуется информация о глобальной скорости сходимости для модифицированных динамик, на которую также влияют собственные значения на границах. Напомним, что контроль над плотностью на границах ослабевает, так как собственные значения, как правило, подвержены сильным флуктуациям вблизи границ.

Методы, обеспечивающие хороший контроль над локальной плотностью, были разработаны в наших предыдущих работах о матрицах Вигнера [55]-[57], но поведение на границах там не было оптимальным. Тем не менее, в [6] нам удалось доказать соотношение (1.66) достаточно сложным способом, основанным на некоторых улучшениях оценок из [55]-[57]. В [9] нами был найден более прямой способ контроля локальной плотности и более эффективный метод доказательства соотношения (1.66); его улучшенная версия получена в [8], мы также приведем набросок в разделе 2. Наиболее сильный результат [7], который будет изложен в п. 2.4, устанавливает соотношение (1.66) фактически для $2 \varepsilon=1$.

Заметим, что эти доказательства проходят также и для обобщенных матриц Вигнера, дисперсии которых удовлетворяют соотношению (1.17). В этом случае мы доказываем локальный полукруговой закон вплоть до фактически наименьших возможных порядков энергии $N^{-1}$ (по модулю множителей вида $\log N)$. Этого достаточно для доказательства соотношения (1.66), и, тем самым, мы можем применить нашу общую теорему для доказательства универсальности локальных статистик для этих матриц. Более трудным случаем являются ленточнъе матрицы Вигнера (1.18), для которых, грубо говоря, $\sigma_{i j}^{2}=0$ при $|i-j|>W$ для некоторого $W \ll N$. Для этого случая мы установили в [9], [8] локальный полукруговой закон до порядков энергии $W^{-1}$, но этого не достаточно для доказательства соотношения (1.66) в случае, когда $W$ намного меньше $N$ (в случае $W \geqslant N^{1-\delta}$ с некоторым малым $\delta$ метод по-прежнему работает).

1.6.3. Удаление гауссовых конволюций. I. Обратный тепловой поток. В двух предыдущих пунктах мы обсуждали методы доказательства универсальности внутри спектра для матриц с малыми гауссовыми конволюциями. Метод работы [14] (п. 1.6.1) требует наличия лишь очень малой гауссовой компоненты (с дисперсией $N^{-1+\varepsilon}$ ), но работает только в эрмитовом случае. Гидродинамический метод [7] (п. 1.6.2) работает в общем случае (более ранние версии [9], [8] предполагали наличие большей гауссовой компоненты с дисперсией $\sim N^{-\xi}$ ). Оба метода, тем не менее, нуждаются в дополнительном рассуждении с возмущениями для удаления этой малой гауссовой компоненты. 
Было разработано два независимых подхода для удаления ограничения на гауссову делимость. Первый подход использует рассуждение с обратным mеnловым потоком, которое впервые появилось в [16], а затем было улучшено в [6; раздел 6]. Преимущество этого метода заключается в том, что он позволяет доказывать универсальность для фиксированной энергии, как сформулировано в (1.35), и, кроме того, этот метод достаточно прост. Недостаток заключается в том, что он требует некоторых условий гладкости на распределение $\nu$ величин $\sqrt{N} h-$ нормированных элементов матриц Вигнера. Мы всегда предполагаем, что $\nu$ убывает субэкспоненциально, т. е. существуют константы $C, \vartheta>0$ такие, что для любого $s$ имеет место неравенство

$$
\int \mathbf{1}(|x| \geqslant s) \mathrm{d} \nu(x) \leqslant C \exp \left(-s^{\vartheta}\right) .
$$

Второй подход, появившийся немного позднее первого, состоит в применении теоремы о сравнении для функиий Грина и рассуждения с возмущениями, использующего условие четырех моментов. Преимуществом этого метода является то, что он работает для любого распределения с условием субэкспоненциального убывания (1.67). Недостаток заключается в том, что он позволяет доказать (1.35) лишь после некоторого усреднения по $E$.

Условие четырех моментов было изначально введено Тао и Ву [17] и затем использовано в [64], [69] в их исследовании возмущений собственных значений, сосредоточенном на совместных статистиках собственных значений с фиксированными индексами. В разделе 4 мы изложим наш подход [9], основанный на возмущении резольвенты. Наш результат не только определяет фиксированные собственные значения, но также является достаточно сильным для определения локальных статистик, а его доказательство намного проще, чем в [17] (более подробные пояснения даны в п. 1.6.4).

В настоящем пункте мы кратко изложим метод обратного теплового потока, а в следующем пункте приведем объяснение сравнительных принципов четырех моментов.

Для простоты изложения мы рассмотрим эрмитов случай, однако мы подчеркиваем, что данный метод, в отличие от метода, описанного в п. 1.6.1, применим и к другим классам симметрии. Рассмотрим процесс Орнштейна-Уленбека, определяемый формулой (1.55), который сохраняет фиксированной дисперсию $1 / 2$, и пусть $\gamma(\mathrm{d} x)=\gamma(x) \mathrm{d} x:=\pi^{-1 / 2} e^{-x^{2}} \mathrm{~d} x$ обозначает обратимую меру для этого процесса. Пусть далее $\nu_{0}(\mathrm{~d} x)=u(x) \gamma(\mathrm{d} x)$ - начальная мера вещественных и мнимых частей нормированных элементов матрицы Вигнера $\widehat{H}$ (заметим, что на протяжении большей части этой работы $\nu$ обозначает плотность величины $\sqrt{N} h_{i j}$, в то время как в этом рассуждении мы ввели обозначение $\nu_{0}$ для общего распределения величин $\sqrt{N} \operatorname{Re} h_{i j}$ и $\sqrt{N} \operatorname{Im} h_{i j}, i \neq j$ ). Рассмотрим теперь действие процесса Орнштейна-Уленбека (1.55) на матричные элементы, т. е. рассмотрим $H_{t}$ - решение уравнения (1.54). Для фиксированного $t>0$ распределение матрицы $H_{t}$ задается формулой

$$
e^{-t / 2} \widehat{H}+\left(1-e^{-t}\right)^{1 / 2} V
$$

где $V$ - матрица ГУА, независимая от $\widehat{H}$. Распределение вещественных и мнимых частей элементов матрицы $H_{t}$ тогда задается функцией $u_{t}(x) \gamma(\mathrm{d} x)$, где 
$u_{t}$ - решение уравнения (1.55) с начальными данными $u_{0}=u$ (строго говоря, эти формулы верны лишь для внедиагональных элементов, элементы на диагонали имеют в два раза большую дисперсию и подвергаются несколько иному потоку Орнштейна-Уленбека).

Основное наблюдение заключается в том, что рассуждения из п. 1.6.1 или п. 1.6.2 гарантируют наличие синус-ядра для любой эрмитовой матрицы, содержащей гауссову компоненту с дисперсией $N^{-1+\varepsilon}$ или $N^{-\xi}$ соответственно (метод п. 1.6.1 применим лишь в эрмитовом случае, а метод п. 1.6.2 работает и в общем случае). Для данной матрицы Вигнера $\widehat{H}$ нам не обязательно сравнивать $\widehat{H}$ с ее гауссовой конволюцией (1.68); достаточно найти другую матрицу Вигнера $\widetilde{H}$, для которой с очень большой точностью имеет место соотношение

$$
\widehat{H} \approx e^{-t / 2} \widetilde{H}+\left(1-e^{-t}\right)^{1 / 2} V .
$$

В действительности, матрицу $\widetilde{H}$ можно даже выбрать зависящей от $t$. Следующая лемма показывает, что любая матрица Вигнера $\widehat{H}$ с достаточно гладким распределением может быть сколь угодно хорошо приближена гауссово делимыми матрицами вида (1.69), если $t \sim N^{-\delta}$ для некоторого $\delta>0$.

Мы предположим, что начальная плотность положительна, т. е. $u(x)>0$, и может быть представлена в виде

$$
u(x)=e^{-V(x)}, \quad \text { где } \sum_{j=1}^{2 K}\left|V^{(j)}(x)\right| \leqslant C_{K}\left(1+x^{2}\right)^{C_{K}}
$$

для любого $K \in \mathbb{N}$ с достаточно большими константами $C_{K}$. Более того, мы предположим, что начальное распределение $\mathrm{d} \nu_{0}=u \mathrm{~d} \gamma$ отдельных элементов убывает субэкспоненциально (см. (1.67)). Следующее аппроксимационное утверждение является ключевой технической леммой.

ЛЕмма 1.5 [6; предложение 6.1]. Предположим, что для некоторого $K>0$ мера $\mathrm{d} \nu_{0}=u \mathrm{~d} \gamma$ удовлетворяет условиям (1.67) $и$ (1.70). Тогда имеется малая константа $\alpha_{K}$, зависящая от $K$, такая, что для любого $t \leqslant \alpha_{K}$ существует плотность вероятности $g_{t}$ с нулевым математическим ожиданием и дисперсией $1 / 2$, для которой выполнено неравенство

$$
\int\left|e^{t A} g_{t}-u\right| \mathrm{d} \gamma \leqslant C t^{K}
$$

где $C>0$ - некоторая константа, зависящая от $K$.

Более того, пусть $\mathscr{A}=A^{\otimes n}, F=u^{\otimes n}$ для некоторого $n \leqslant C N^{2}$. Обозначим $G_{t}=g_{t}^{\otimes n}$. Тогда

$$
\int\left|e^{t \mathscr{A}} G_{t}-F\right| \mathrm{d} \gamma^{\otimes n} \leqslant C N^{2} t^{K}
$$

для некоторой константы $C>0$, зависящей от $K$.

НАБРОСОК ДОКАЗАТЕЛЬСТВА. Для данного $u$ необходимо решить уравнение

$$
e^{A t} g_{t}=u,
$$


т. е. формально мы имеем $g_{t}=e^{-A t} u$. Однако действие оператора $e^{-A t}$ заключается в применении теплового потока (с действием процесса Орнштейна-Уленбека) в обратном времени, и этот оператор, как правило, не определен, если $u$ не является аналитической функцией. Тем не менее, мы можем определить приближенное решение обратного уравнения теплопроводности, т. е. положить

$$
g_{t}:=\left(I-A t+\frac{t^{2} A^{2}}{2 !}-\cdots+\frac{(-t A)^{K-1}}{(K-1) !}\right) u .
$$

Так как $A$ - дифференциальный оператор второго порядка, а функция $u$ достаточно гладкая, это выражение определено корректно, более того,

$$
e^{A t} g_{t}=O\left(t^{K} A^{K} u\right)=O\left(t^{K}\right)
$$

Это доказывает неравенство (1.71), а неравенство (1.72) непосредственно вытекает из него.

Вооружившись леммой 1.5, мы можем доказать универсальность синус-ядра в виде (1.35) для любой эрмитовой матрицы Вигнера, удовлетворяющей условиям (1.67) и (1.70) при любом фиксированном $|E|<2$. Пусть $n \sim N^{2}$ - число независимых процессов Орнштейна-Уленбека, необходимых для порождения матричных элементов. Выбирая $K$ достаточно большим, мы можем сравнить две меры $e^{t \mathscr{A}} G_{t}$ и $F$ в полной вариационной норме; для любой наблюдаемой функции $J: \mathbb{R}^{n} \rightarrow \mathbb{R}$ матричных элементов мы имеем

$$
\left|\int J\left(e^{t \mathscr{A}} G_{t}-F\right) \mathrm{d} \gamma^{\otimes n}\right| \leqslant\|J\|_{\infty} C N^{2} t^{K} .
$$

Для доказательства свойства (1.35) необходимо выбрать наблюдаемые $J$, зависимость которых от матричных элементов выражается через собственные значения и которые задают локальные корреляционные функции. Легко видеть, что для таких $J$ норма $\|J\|_{\infty}$ может расти не быстрее чем полиномиально по $N$. При этом мы всегда можем выбрать достаточно большое $K$, чтобы компенсировать выбор $t=N^{-\delta}$. Так как синус-ядро применимо для распределения $e^{t \mathscr{A}} G_{t}$ с $t=N^{-1+\varepsilon}$ (п. 1.6.1) или $t=N^{-\xi}$ (п. 1.6.2), оно также применимо для меры Вигнера $F$.

Для симметрических матриц рассуждение с обратным тепловым потоком повторяется дословно. Однако затем мы можем применить лишь результаты п. 1.6.2 для получения универсальности при малых временах; в частности, величину $E$ в (1.35) необходимо усреднить.

1.6.4. Удаление гауссовых конволюций. II. Теорема сравнения для функций Грина. Пусть $H$ и $H^{\prime}$ - два ансамбля Вигнера, для которых первые четыре момента распределений $\nu$ и $\nu^{\prime}$ индивидуальных собственных значений совпадают:

$$
m_{j}=m_{j}^{\prime}, \quad j=1,2,3,4,
$$

где

$$
m_{j}:=\int_{\mathbb{R}} x^{j} \mathrm{~d} \nu(x) \quad \text { и } \quad m_{j}^{\prime}:=\int_{\mathbb{R}} x^{j} \mathrm{~d} \nu^{\prime}(x) .
$$


Для комплексных матричных элементов необходимо брать наборы всех $j$-моментов, т. е. $m_{j}$ представляет набор всех $\int_{\mathbb{C}} x^{a} \bar{x}^{b} \mathrm{~d} \nu(x)$ с $a+b=j$. Напомним, что $\nu$ есть распределение величины $\sqrt{N} h_{i j}$. При нашей нормировке матриц Вигнера первый момент всегда равен нулю, а второй момент - единице, так что (1.73) в действительности является условием на третий и четвертый моменты.

Наш основной результат заключается в следующей теореме сравнения для совместного распределения функций Грина. Здесь мы приведем этот результат в несколько упрощенном виде, а более подробное изложение будет дано в разделе 4 .

Теорема 1.6 (теорема сравнения для функций Грина [9; теорема 2.3]). Pacсмотрим две матрицы Вигнера $H$ и $H^{\prime}$ с распределениями индивидуальных собственных значений $\nu$ и $\nu^{\prime}$. Предположим, что для $\nu$ и $\nu^{\prime}$ выполнены условия (1.67) и (1.73). Обозначим $G(z)=(H-z)^{-1} u G^{\prime}(z)=\left(H^{\prime}-z\right)^{-1}$. Зафиксируем $k$ и предположим, что функиия $F: \mathbb{R}^{k} \rightarrow \mathbb{R}$ удовлетворяет условию

$$
\sup _{x \in \mathbb{R}^{k}}\left|\nabla^{j} F(x)\right| \leqslant N^{\varepsilon^{\prime}}, \quad 0 \leqslant j \leqslant 5 .
$$

Зафиксируем малые параметры $\kappa и \varepsilon$. Тогда для достаточно малого $\varepsilon^{\prime}$ найдется константа $c_{0}>0$ такая, что для любых иелых значений $\ell_{1}, \ldots, \ell_{k}$ u спектральных параметров $z_{j}^{m}=E_{j}^{m} \pm i \eta, 1 \leqslant j \leqslant \ell_{m}, m=1, \ldots, k$, c $E_{j}^{m} \in[-2+\kappa, 2-\kappa] u \eta \geqslant N^{-1-\varepsilon}$ ми имеем

$$
\left|\mathrm{E} F\left(\frac{1}{N} \operatorname{Tr}\left[\prod_{j=1}^{\ell_{1}} G\left(z_{j}^{1}\right)\right], \ldots, \frac{1}{N} \operatorname{Tr}\left[\prod_{j=1}^{\ell_{k}} G\left(z_{j}^{k}\right)\right]\right)-\mathrm{E}^{\prime} F\left(G \rightarrow G^{\prime}\right)\right| \leqslant N^{-c_{0}}
$$

Здесь мы использовали сокращенную запись $F\left(G \rightarrow G^{\prime}\right)$ для обозначения функции $F$ с теми же аргументами, что и в первой части формулы $(1.75)$, но с заменой $G$ заменено на $G^{\prime}$.

В действительности, условие (1.73) можно ослабить, потребовав лишь, чтобы третий и четвертый моменты были близки:

$$
m_{j}=m_{j}^{\prime}, \quad j=1,2, \quad \text { и } \quad\left|m_{3}-m_{3}^{\prime}\right| \leqslant N^{-1 / 2-\delta}, \quad\left|m_{4}-m_{4}^{\prime}\right| \leqslant N^{-\delta}
$$

для некоторого $\delta>0$. Тогда неравенство (1.75) по-прежнему имеет место, но $\varepsilon$ и $\varepsilon^{\prime}$ должны быть достаточно малыми, в зависимости от $\delta$, и $c_{0}$ также будет зависеть от $\delta$. Точная оценка будет дана в теореме 4.1 .

Другими словами, при условии совпадения четырех моментов ансамблей Вигнера, математические ожидания следов любых комбинаций резольвентных произведений совпадают, если спектральные параметры в резольвентах находятся на расстоянии по крайней мере $\eta=N^{-1-\varepsilon}$ от вещественной оси. Такое малое расстояние соответствует спектральному разрешению до размеров порядка $N^{-1-\varepsilon}$, т. е. оно позволяет определять локальные корреляционные функции индивидуальных собственных значений. Простое алгебраическое тождество позволяет выразить корреляционные функции через следы резольвент. 
Например, одноточечная корреляционная функция (плотность) в масштабе $\eta$ допускает следующее приближение:

$$
p_{N}^{(1)}(E) \sim \frac{1}{\pi N} \operatorname{Im} \operatorname{Tr} G(E+i \eta)=\frac{1}{2 \pi}\left[\frac{1}{N} \operatorname{Tr} G(E+i \eta)-\frac{1}{N} \operatorname{Tr} G(E-i \eta)\right],
$$

а более высокие точечные корреляционные функции включают многочлены более высокого порядка от резольвент. Таким образом, теорема 1.6 непосредственно сравнивает корреляционные функции (точное утверждение будет дано в теореме 4.2). Заметим, что взятие следов не является существенным в (1.75), схожий принцип сравнения работает также для матричных элементов резольвент (точную формулировку см. в [9]). Фактически, доказательство теоремы 1.6 опирается на рассуждение с возмущениями, непосредственно включающее матричные элементы резольвенты. Ключевой составляющей является сильная форма локального полукругового закона, которая позволяет непосредственно оценить величины $G_{i i}$ и $G_{i j}$ с $i \neq j$, а не только нормализованный след $m(z)=\frac{1}{N} \sum_{i} G_{i i}$ (более сильный результат приведен в $(2.35)-(2.36)$ и $(2.111)$ ).

Похожая теорема для собственных значений была ранее доказана в [17]. Пусть $\lambda_{1}<\cdots<\lambda_{N}$ и $\lambda_{1}^{\prime}<\cdots<\lambda_{N}^{\prime}-$ собственные значения матриц $H$ и $H^{\prime}$ соответственно. Следующая теорема утверждает, что совместные распределения любых наборов из $k$ собственных значений в масштабе $1 / N$ весьма близки друг к другу.

ТЕоремА 1.7 (теорема о четырех моментах для собственных значений [17; теорема 15]). Пусть $H$ и $H^{\prime}$ - две матрици Вигнера. Предположим, что для распределений их отдельных собственных значений $\nu$ u $\nu^{\prime}$ выполнены условия (1.67) и (1.73). Тогда для любых достаточно малых положителъных $\varepsilon$ и $\varepsilon^{\prime}$, для любой функиии $F: \mathbb{R}^{k} \rightarrow \mathbb{R}$, удовлетворяющей условию (1.74), и для любых $k$ индексов $i_{1}, \ldots, i_{k} \in[\varepsilon N,(1-\varepsilon) N]$, выбранных вдали от грании, спектра, ми имеем

$$
\left|\mathrm{E} F\left(N \lambda_{i_{1}}, \ldots, N \lambda_{i_{k}}\right)-\mathrm{E}^{\prime} F\left(N \lambda_{i_{1}}^{\prime}, \ldots, N \lambda_{i_{k}}^{\prime}\right)\right| \leqslant N^{-c_{0}}
$$

при некотором $c_{0}>0$. Условие (1.73) можно ослабить до (1.76), но тогда $c_{0}$ будет зависеть от $\delta$.

Заметим, что аргументы в формуле (1.77) увеличены на множитель $N$, а функция $F$ может быть сосредоточена в масштабе $N^{-\varepsilon^{\prime} / 5}$, так что результат является достаточно точным для того, чтобы определить корреляции собственных значений в масштабе $N^{-1-\varepsilon^{\prime} / 5}$, т. е. даже несколько меньшем, чем расстояния между собственными значениями. Таким образом, теоремы 1.6 и 1.7 дают доказательство универсальности внутри спектра для матрицы Вигнера $H$, как только найдена другая матрица $H^{\prime}$ с теми же четырьмя моментами, для которой универсальность уже установлена. В эрмитовом случае матрицы ГУА или более общие гауссово делимые матрицы (1.53) являются хорошими кандидатами для сравнительных ансамблей $H^{\prime}$. Согласование с матрицей ГУА требует совпадения третьих и четвертых моментов: $m_{3}=0, m_{4}=3$. Так как расположение собственных значений ГУА известно достаточно точно [92], [93], 
то соотношение (1.77) можно интерпретировать как предел корреляционных функций (см. (1.35)) даже для фиксированной энергии $E$. Если необходимо получить лишь предельное распределение промежутков (1.36), а не (1.35), то для сравнения можно непосредственно использовать гауссово делимую матрицу (1.53). Легко проверяется [94], что для любого вероятностного распределения $\nu$ с $m_{1}=0$ и $m_{2}=1$, носитель которого содержит по крайней мере три точки, имеется распределение с гауссовой компонентой порядка один и теми же первыми четырьмя моментами. Следовательно, матрица $H$ может быть сравнена с гауссово делимой матрицей, для которой универсальность установлена в [13]. Используя результат [14] об универсальности эрмитовых матриц Вигнера с малой гауссовой конволюцией (содержащийся в п. 1.6.1), а также тот факт, что точное совпадение моментов (1.73) можно ослабить до (1.76), можно сравнить любую матрицу Вигнера $H$ с ее конволюцией Орнштейна-Уленбека (1.53) с очень коротким временем $t \sim N^{-1+\varepsilon}$. Это позволяет опустить условие $m_{3}=0$ и условие, что носитель содержит хотя бы три точки, и доказать универсальность корреляционных функций для любой матрицы Вигнера в смысле (1.35) после небольшого усреднения по $E$ [18]. Единственным техническим условием является субэкспоненциальное убывание функции $\nu$ (см. (1.67)).

В симметрическом случае отсутствует аналог результата Йоханссона [13] (если не использовать результаты [16]), и единственным доступным ансамблем для сравнения является ГОА. Тогда из теоремы 1.7 вытекает [17] универсальность для симметрических матриц Вигнера, первые четыре момента распределения отдельных собственных значений которых согласуются с моментами ГОА в смысле (1.76).

Внимательный читатель мог заметить небольшое различие между наблюдаемыми в (1.77) и локальными корреляционными функциями (1.35). Хотя и те и другие определяют структуру в масштабе $1 / N$, в соотношении (1.77) индексы собственных значений являются фиксированными, в то время как в (1.35) фиксированным является их расположение. Грубо говоря, соотношение (1.77) дает ответ на вопрос типа "где находятся $N / 2$-е и $(N / 2-1)$-е собственные значения?". Локальные корреляционные функции дают ответ на вопрос "какова вероятность одновременного нахождения одного собственного значения в $E$ и другого в $E^{\prime}=E+\alpha / N ? "$. Эти два вопроса можно связать друг с другом, только если имеется некоторая априорная информация о расположении собственных значений.

До появления работы [17], за исключением случая ГУА [92], [93], ни для каких других ансамблей не удавалось определить расположение собственных значений с точностью $N^{-1+c_{0}}$ для малых $c_{0}$. Именно такая точность необходима для того, чтобы перевести соотношение (1.77) в (1.35) при фиксированном $E$. Используя вариант теоремы 1.7 , в котором речь идет о совпадении лишь трех моментов, можно определить расположение собственных значений любого ансамбля Вигнера с необходимой точностью, при условии, что третий момент обращается в нуль (т. е. совпадает с ГУА). Имея эту информацию, можно далее добиться совпадения четвертого момента путем выбора соответствующей гауссово делимой матрицы. Это возможно, если носитель исходного распределения содержит хотя бы три точки. Именно поэтому соотношение (1.35) было в итоге доказано в [17] при условии, что третий момент обращается в нуль, а носитель 
содержит хотя бы три точки. Если смириться с тем, что соотношение (1.35) будет доказано лишь после некоторого усреднения по $E$, то необходимо значительно меньше информации о расположении собственных значений, и эта информация, как правило, может быть извлечена из локального полукругового закона.

В действительности отслеживание индивидуальных собственных значений может быть трудной задачей; заметим, что теорема 1.7 сама по себе не дает сходимости корреляционных функций - для этого по-прежнему необходима информация о расположении $i$-го собственного значения. С другой стороны, теорема 1.7 содержит информацию о собственных значениях с фиксированными индексами, которая не содержится в теореме 1.6. Мы отметим, что использование локального полукругового закона существенно в обеих теоремах.

Основная причина, по которой доказательство теоремы 1.6 является более коротким, заключается в том, что корреляционные функции можно определить из наблюдаемых, включающих следы резольвент $(H-z)^{-1}$, где $\operatorname{Im} z \sim N^{-1-\varepsilon}$. Эти резольвенты допускают априорные оценки порядка $|\operatorname{Im} z|^{-1} \leqslant N^{1+\varepsilon}$, так что формулы для возмущений, включающие резольвенты, не претерпевают разрыв. С другой стороны, индивидуальные собственные значения, отслеживаемые при помощи теоремы 1.7, могут производить резонансы, приводящие к тому, что некоторые члены могут в принципе становиться бесконечными (мы приведем набросок доказательства теоремы 1.7 в разделе 4). Хотя общим свойством ансамблей Вигнера является отталкивание уровней, а оно сильно подавляет резонансы, прямое доказательство отталкивания уровней является непростой задачей. В действительности, наиболее технически сложная оценка из [17] - это оценка нижних хвостов распределения промежутков (теорема 17 в [17]). Она утверждает, что для любого $c_{0}>0$ найдется $c_{1}$ такое, что

$$
\mathrm{P}\left(\lambda_{i+1}-\lambda_{i} \leqslant N^{-1-c_{0}}\right) \leqslant N^{-c_{1}},
$$

если индекс $i$ удовлетворяет условию $\varepsilon N \leqslant i \leqslant(1-\varepsilon) N$.

1.6.5. Сводка новых результатов об универсальности внутри спектра. Даже хорошо подготовленному читателю последние достижения могут показаться несколько запутанными, так как имеется много работ, где универсальность матриц Вигнера устанавливается при различных условиях и разными методами. Взаимосвязи между ними не всегда хорошо отражены в недавних публикациях, так как данное направление по-прежнему развивается. В этом пункте мы постараемся сориентировать читателя в содержании последних публикаций.

Как уже отмечалось во введении, основным направляющим принципом во всех этих доказательствах универсальности локальных статистик собственных значений является сравнение локальных статистик матрицы Вигнера с другой матрицей, содержащей гауссову компоненту. Более точно, наш подход включает три основных шага:

1) локальный полукруговой закон;

2) универсальность для гауссово делимых ансамблей, т. е. таких, в которых вероятностный закон для матричных элементов содержит малую гауссову компоненту;

3) универсальность для общих ансамблей; использование приближения гауссово делимым ансамблем для удаления малой гауссовой компоненты. 
Нам было ясно с самого начала, что первым шагом в любом доказательстве универсальности должен быть хороший локальный полукруговой закон. В действительности, все доказательства универсальности существенно используют особенности оценок, которые можно получать из локального полукругового закона. Далее мы дадим обзор существующих результатов в соответствии с тремя шагами, приведенными выше.

Хотя в настоящее время имеется достаточно простое доказательство локального полукругового закона в наименьшем масштабе $\eta \sim 1 / N$, к нему приходили лишь постепенно. В нашей первой работе [55] была дана верхняя ощенка локальной плотности фактически вплоть до оптимального порядка энергии $\eta \sim(\log N) / N$, но локальный полукруговой закон был доказан лишь в масштабе $\eta \gg N^{-2 / 3}$. Во второй работе [56] локальный полукруговой закон был доказан нами вплоть до масштаба $\eta \geqslant(\log N)^{8} / N$ - почти оптимального, но все еще включающего логарифмический множитель. Хвостовые вероятности нарушения локального полукругового закона также были далеки от оптимальных. Оба эти недостатка были устранены в работе [57] (см. теорему 1.11 ниже), где, кроме того, был получен оптимальный делокализационный результат для собственных векторов (теорема 2.22). В первой работе [55] мы налагали сильное условие (гауссова) убывания, а также требовали некоторое свойство выпуклости распределения отдельных собственных значений, которое влечет их концентрацию (при помощи неравенств Браскампа-Лееба или логарифмических неравенств Соболева). Эти технические условия затем были устранены, а условие гауссова убывания было заменено на субэкспоненциальное убывание. Наконец, в работе [9] и ее улучшенной версии [8] мы получили значительно более сильную оценку ошибок для локального полукругового закона (см. (1.4)-(1.7)), однако эти оценки по-прежнему ухудшались на границах. Оптимальный результат [7], который мы называем сильным локальным полукруговым законом (теорема 2.19), имеет место равномерно по параметру энергии.

Что касается шага 2), основной особенностью здесь является то, что гауссова компонента позволяет выявить универсальное поведение. Имеется два метода использования этого соображения:

i) контурное интегральное представление, следуя работам Йоханссона [13] и Бен Аруса и Пеше [85]; однако этот метод доступен лишь для эрмитова случая (или для случая комплексных выборочных ковариаций, см. [85]);

ii) гидродинамический подход, при котором малая гауссова компонента (или, эквивалентно, эволюция процесса Орнштейна-Уленбека за малое время) уже приводит систему в локальное равновесие; этот подход работает для всех ансамблей, включая симметрические, эрмитовы симплектические и ансамбли выборочных ковариаций; он также дает концептуальное понимание того, что универсальность происходит из броуновского движения Дайсона.

Оба подхода требуют наличия хорошего локального полукругового закона. Кроме того, в ранних работах [16] и [6] по гидродинамическим методам также требовалось выполнение логарифмического неравенства Соболева для распределения $\nu$ индивидуальных матричных элементов - чтобы вывести соотношение (1.66) из локального полукругового закона. В [8] от этого последнего 
условия удалось избавиться путем использования усиленного локального полукругового закона, который привел к более простому и мощному доказательству соотношения (1.66) (теорема 2.7 ниже). Наконец, сильный локальный полукруговой закон, полученный в [7] (теорема 2.19 ниже), дал наилучший показатель $2 \varepsilon=1$ в (1.66).

Подводя итог обсуждению первых двух шагов, мы получаем универсальность внутри спектра для обобщенных матриц Вигнера (1.17) с малой гауссовой конволюцией при единственном условии субэкспоненциального убывания. Это условие можно далее ослабить до полиномиального убывания большой степени, но детали нами проработаны не были. Более того, хотя обобщение сильного локального полукругового закона на выборочные ковариационные матрицы получается непосредственно, детали здесь также не проработаны (более раннее подробное доказательство в [6] требовало выполнения логарифмического неравенства Соболева).

Первые два шага предоставляют большой класс матричных ансамблей с универсальными локальными статистиками. На шаге 3) остается аппроксимировать произвольные матричные ансамбли матрицами, полученными на первых двух шагах, с сохранением локальных статистик. Это аппроксимационный шаг можно совершить двумя способами:

a) при помощи обратного теплового потока;

b) при помощи теоремы сравнения для функций Грина.

Рассуждение с обратным тепловым потоком особенно просто, но оно требует гладкости распределения отдельных элементов. Этот подход использовался в [14], [16] и [6] и привел к доказательству универсальности для всех упомянутых выше ансамблей при условии гладкости. От условия гладкости затем удалось избавиться в [9], где была впервые доказана теорема сравнения для функций Грина. K сожалению, нам по-прежнему требовалось выполнение логарифмического неравенства Соболева, и универсальность была установлена лишь для матриц, носитель распределения которых содержал хотя бы три точки.

Более сильная версия локального полукругового закона была доказана в [8], и все условия на гладкость и носитель распределения были устранены. В итоге, в [8] мы получили универсальность внутри спектра для корреляционных функций (1.35) и распределения промежутков (1.36) для всех классических ансамблей Вигнера (включая обобщенные матрицы Вигнера (1.17)). Универсальность в (1.35) понимается после небольшого усреднения по параметру энергии $E$. Единственным условием на распределение $\nu$ отдельных элементов является его субэкспоненциальное убывание (1.67).

Подход Тао и Ву [17] использует похожую стратегию, включающую шаги 1)-3), упомянутые в начале этого пункта. Для шага 2) универсальность эрмитовых матриц Вигнера и комплексных выборочных ковариационных матриц уже была доказана Йоханссоном [13] и Бен Арусом и Пеше [85]. Шаг 3) вытекает из теоремы Тао-Ву о четырех моментах (теорема 1.7), доказательство которой использует локальный полукруговой закон - шаг 1). Это приводит к доказательству универсальности для эрмитовых матриц Вигнера [17] и комплексных выборочных ковариационных матриц [69], удовлетворяющих условию, что носитель распределения содержит по крайней мере три точки (кроме 
того, для получения результата при фиксированной энергии в (1.35) требуется также обращение в нуль третьего момента). В симметрическом случае требуется совпадение первых четырех моментов. Подход Тао-Ву также может быть применен для доказательства универсальности на границах спектра [64]. В [69] субэкспоненциальное убывание было заменено условием достаточно сильного полиномиального убывания. От ограничений на носитель и на третий момент можно избавиться путем совместного использования результатов [17] и результата, вытекающего из нашего подхода [14]. Это привело к доказательству универсальности эрмитовых матриц [18] для любого распределения, включая меру Бернулли. С другой стороны, для эрмитовых матриц в этом подходе требуется постоянство дисперсий матричных элементов.

Исторически первая работа Тао-Ву [17] на тему универсальности появилась сразу после работы [14] об универсальности эрмитовых матриц. Общей составляющей подходов [14] и [17] был локальный полукруговой закон и оценки делокализации собственных функций, которые фактически были приведены в [56], [57], но в силу некоторых технических условий они затем были передоказаны в [17]. Локальный полукруговой закон для выборочных ковариационных матриц был впервые доказан в [6], а затем несколько иной его вариант появился в [69] с некоторыми изменениями в технических предположениях, связанными с приложениями.

Условие четырех моментов впервые появилось в теореме о четырех моментах Тао-Ву [17] (теорема 1.7) и было использовано в теореме сравнения для функций Грина [9] (теорема 1.6). Теорема о четырех моментах касается индивидуальных собственных значений и поэтому непосредственно содержит информацию о распределении промежутков между собственными значениями. Для того чтобы перевести эту информацию в корреляционные функции, требуется информация о расположении индивидуальных собственных значений для сравнительного ансамбля. С другой стороны, теорема сравнения для функций Грина может быть использована непосредственно для сравнения корреляционных функций, но дает меньше информации об индивидуальных собственных значениях. В то же время, стандартное рассуждение с принципом включения-исключения (аналогичное приведенному в (1.38)) также дает универсальность для распределения промежутков. Так как индивидуальные собственные значения подвержены флуктуациям, а функции Грина более стабильны, это объясняет, почему доказательство теоремы о четырех моментах достаточно сложно, в то время как доказательство теоремы сравнения для функций Грина весьма просто. Более того, теорема сравнения для функций Грина предоставляет не только спектральную информацию, но также и информацию о матричных элементах.

1.6.6. Новые результаты об универсальности на границах. Напомним, что $\lambda_{N}$ обозначает наибольшее собственное значение случайной матрицы. Функции распределения вероятностей для $\lambda_{N}$ в случае классических гауссовых ансамблей были найдены Трейси и Уидомом [65], [66] в следующем виде:

$$
\lim _{N \rightarrow \infty} \mathrm{P}\left(N^{2 / 3}\left(\lambda_{N}-2\right) \leqslant s\right)=F_{\beta}(s),
$$


где функция $F_{\beta}(s)$ может быть вычислена в терминах уравнений Пенлеве, а значения $\beta=1,2,4$ соответствуют стандартным классическим ансамблям. Имеется убеждение, что распределение собственного значения $\lambda_{N}$ универсально и не зависит от гауссовой структуры. Совместное применение сильного локального полукругового закона (теоремы 2.19) и версии теоремы сравнения для функций Грина (теоремы 1.6), адаптированной к границам спектра, дает следующий вариант универсальности для экстремальных собственных значений.

Теорема 1.8 (универсальность экстремальных собственных значений [7; теорема 2.4]). Пусть даны две обобщенные матрицы Вигнера $H^{(v)}$ и $H^{(w)}$ размера $N \times N$ с матричными элементами $h_{i j}$, задаваемыми случайными величинами $N^{-1 / 2} v_{i j}$ и $N^{-1 / 2} w_{i j}$ соответственно, причем $v_{i j}$ и $w_{i j}$ удовлетворяют условию субэкспоненциального убывания (1.67) равномерно по всем $i, j$. Пусть $\mathrm{P}^{\mathbf{v}}$ u $\mathrm{P}^{\mathbf{w}}$ обозначают вероятности, $a \mathrm{E}^{\mathbf{v}} u \mathrm{E}^{\mathbf{w}}$ - математические ожидания по отношению $к$ этим наборам случайных величин. Если первые два момента для $v_{i j}$ и $w_{i j}$ совпадают, m.е.

$$
\mathrm{E}^{\mathbf{v}} \bar{v}_{i j}^{l} v_{i j}^{u}=\mathrm{E}^{\mathbf{w}} \bar{w}_{i j}^{l} w_{i j}^{u}, \quad 0 \leqslant l+u \leqslant 2,
$$

то найдутся такие $\varepsilon>0 u \delta>0$, зависящие от $\vartheta$ в (1.67), что для любого $s \in \mathbb{R}$ выполнены неравенства

$$
\begin{aligned}
& \mathrm{P}^{\mathbf{v}}\left(N^{2 / 3}\left(\lambda_{N}-2\right) \leqslant s-N^{-\varepsilon}\right)-N^{-\delta} \\
& \quad \leqslant \mathrm{P}^{\mathbf{w}}\left(N^{2 / 3}\left(\lambda_{N}-2\right) \leqslant s\right) \leqslant \mathrm{P}^{\mathbf{v}}\left(N^{2 / 3}\left(\lambda_{N}-2\right) \leqslant s+N^{-\varepsilon}\right)+N^{-\delta},
\end{aligned}
$$

где $N$ достаточно велико и не зависит от s. Аналогичный результат имеет место и для наименъшего собственного значения $\lambda_{1}$.

Теорема 1.8 может быть обобщена на конечные корреляционные функции экстремальных собственных значений. Например, мы имеем следующее обобщение соотношений (1.81):

$$
\begin{aligned}
\mathrm{P}^{\mathbf{v}}( & \left.N^{2 / 3}\left(\lambda_{N}-2\right) \leqslant s_{1}-N^{-\varepsilon}, \ldots, N^{2 / 3}\left(\lambda_{N-k}-2\right) \leqslant s_{k+1}-N^{-\varepsilon}\right)-N^{-\delta} \\
& \leqslant \mathrm{P}^{\mathbf{w}}\left(N^{2 / 3}\left(\lambda_{N}-2\right) \leqslant s_{1}, \ldots, N^{2 / 3}\left(\lambda_{N-k}-2\right) \leqslant s_{k+1}\right) \\
& \leqslant \mathrm{P}^{\mathbf{v}}\left(N^{2 / 3}\left(\lambda_{N}-2\right) \leqslant s_{1}+N^{-\varepsilon}, \ldots, N^{2 / 3}\left(\lambda_{N-k}-2\right) s_{k+1}+N^{-\varepsilon}\right)+N^{-\delta}
\end{aligned}
$$

для любого фиксированного $k$ и достаточно большого $N$.

Универсальность на границах для матриц Вигнера была впервые доказана при помощи метода моментов Сошниковым [62] (см. также более раннюю работу [63]) для эрмитовых и симметрических ансамблей с симметрическим распределением отдельных элементов $\nu$ (симметричность распределения гарантирует обращение в нуль всех нечетных моментов). Совместное применение метода моментов и многочленов Чебышёва [95] позволило Содину [96], [97] доказать универсальность на границах для ленточных матриц и для некоторого специального класса разреженных матриц.

Устранить предположение симметричности было непросто. Подход [96], [97] работает лишь для ансамблей с симметрическими распределениями. От симметричности удалось частично избавиться в работах [98], [68], затем значительный прогресс был достигнут в [64], где предполагалось лишь совпадение 
первых трех моментов двух ансамблей Вигнера. Другими словами, условие симметричности было заменено на условие обращения в нуль третьего момента для матриц Вигнера. Для специального класса ансамблей - гауссово делимых эрмитовых ансамблей, универсальность на границах была доказана в [86] при единственном условии конечности второго момента. Путем совместного использования методов [86] и [64] тот же результат затем был доказан для всех эрмитовых ансамблей Вигнера с конечным вторым моментом [86].

По сравнению с этими результатами, теорема 1.8 не дает универсальности на границах спектра для ленточных матриц или разреженных матриц [96], [97]. Однако она, в частности, показывает, что для того, чтобы определить распределение наибольшего собственного значения обобщенной матрицы Вигнера, достаточно рассматривать обобщенный ансамбль Вигнера с гауссовым распределением. Так как распределение наибольшего собственного значения гауссовых ансамблей Вигнера задается функцией $F_{\beta}(1.79)$, то из теоремы 1.8 вытекает универсальность на границах спектра для стандартных матриц Вигнера с единственным условием субэкспоненциального убывания. Заметим, что результат теоремы 2.20 можно использовать как входные данные в подходе [86] для доказательства того, что распределения наибольших собственных значений обобщенных эрмитовых ансамблей Вигнера с гауссовыми распределениями задаются функцией $F_{2}$. Однако для ансамблей другого класса симметрии соответствующий результат, позволяющий отождествить распределение наибольшего собственного значения с функцией $F_{\beta}$, отсутствует.

Наконец, мы отметим, что условие субэкспоненциального убывания, накладываемое нашим подходом, хотя и может быть ослаблено, все равно далеко от оптимального для доказательства универсальности на границах [99], [100], [68].

\section{7. Отталкивание уровней и оценка Вегнера для очень малых мас-} штабов. Одну из наших ранних формулировок локального полукругового закона для матриц Вигнера, а именно неравенство

$$
\left|m(z)-m_{\mathrm{sc}}(z)\right| \lesssim \frac{C}{\sqrt{N \eta \kappa}}, \quad \kappa=|| E|-2|,
$$

доказанное в теореме 4.1 из [89], можно превратить в следующую прямую оценку эмпирической плотности:

$$
\left|\varrho_{\eta}(E)-\varrho_{\mathrm{sc}}(E)\right| \lesssim \frac{C}{\sqrt{N \eta \kappa}}, \quad \kappa=|| E|-2| .
$$

Здесь $\varrho_{\eta}$ обозначает эмпирическую плотность $\varrho(x)=\frac{1}{N} \sum_{i} \delta\left(\lambda_{i}-x\right)$, сглаженную в масштабе порядка $\eta$. Этот результат утверждает, что эмпирическая плотность при порядках величин $\eta \gg O(1 / N)$ близка к плотности полукруга. При еще меньших порядках величин $\eta \leqslant O(1 / N)$ эмпирическая плотность флуктуирует, но ее математическое ожидание $\mathrm{E}_{\eta}(E)$ остается ограниченным равномерно по $\eta$. Это и есть оценка типа Вегнера, которая играет ключевую роль в теории локализации для случайных операторов Шрёдингера. В частности, согласно этой оценке, вероятность нахождения хотя бы одного собственного значения в отрезке $I$ размера $\eta=\varepsilon / N$ ограничена величиной $C \varepsilon$ равномерно 
по $N$, причем $\varepsilon \leqslant 1$, т. е. ни одно собственное значение не может постоянно находиться ни в одном уровне энергии $E$. Более того, если собственные значения независимы (т.е. образуют пуассоновский процесс), то вероятность нахождения $n=1,2,3, \ldots$ собственных значений в отрезке $I$ пропорциональна $\varepsilon^{n}$. Для случайных матриц внутри спектра эта вероятность значительно меньше. Это явление известно как отталкивание уровней, а его точная формулировка заключается в следующем.

Теорема 1.9 [57; теоремы 3.4 и 3.5]. Рассмотрим симметрические или эрмитовы матрицы Вигнера с распределением отделъных элементов $\nu$, которое имеет субэкспоненциалъное убъвание. Предположим, что распределение $\nu$ абсолютно непреръвно со строго положительной и гладкой плотностью. Пусть $|E|<2$ и $I=[E-\eta / 2, E+\eta / 2]$ с $\eta=\varepsilon / N$, u nусть $\mathscr{N}_{I}$ обозначает число собственных значений в отрезке I. Тогда для любого фиксированного $n$ имеем

$$
\mathrm{P}\left(\mathscr{N}_{I} \geqslant n\right) \leqslant \begin{cases}C_{n} \varepsilon^{n^{2}} & \text { [эрмитов случай] } \\ C_{n} \varepsilon^{n(n+1) / 2} & \text { [симметрический случай] }\end{cases}
$$

равномерно по $\varepsilon \leqslant 1$ для любого достаточно большого $N$.

Показатели в этой теореме являются оптимальными, что легко вытекает из определителя Вандермонда в формуле совместной плотности вероятности (1.43) для инвариантных ансамблей. Поведение синус-ядра (1.32) указывает на отталкивание уровней и даже дает нижнюю оценку на $\mathrm{P}\left(\mathscr{N}_{I} \geqslant n\right)$, но обычно не для произвольно малых масштабов, так как наличие синус-ядра, как правило, доказывается лишь в слабом пределе (см. (1.35)).

Заметим также, что неравенство (1.78) (теорема 17 из [17]) также представляет собой некоторую оценку на отталкивание уровней, однако показатели здесь не являются оптимальными, и эта оценка не выполнена для произвольно малых масштабов. Тем не менее, неравенство (1.78) не предполагает гладкости распределения, в частности, оно выполнено и для дискретных распределений. Ясно, что, скажем, для распределения Бернулли даже оценка Вегнера (1.84) при $n=1$ не может выполняться для суперэкспоненциально малых порядков величин $\varepsilon \sim 2^{-N^{2}}$.

НАБРОСОК ДОКАЗАТЕЛЬСТВА. Первым шагом в доказательстве является получение верхней оценки для $\mathscr{N}_{I}$. Пусть $H^{(k)}$ обозначает $(N-1) \times(N-1)$-минор матрицы $H$, получаемый удалением $k$-й строки и $k$-го столбца. Пусть $\lambda_{\alpha}^{(k)}$, $\alpha=1, \ldots, N-1$, обозначают собственные значения матрицы $H^{(k)}$, а $\mathbf{u}_{\alpha}^{(k)}$ обозначают ее собственные векторы. Вычисляя $(k, k)$-й диагональный элемент резольвенты $(H-z)^{-1}$, мы легко получаем следующее выражение для $m(z)=m_{N}(z)$ :

$$
m(z)=\frac{1}{N} \sum_{k=1}^{N} \frac{1}{H-z}(k, k)=\frac{1}{N} \sum_{k=1}^{N}\left[h_{k k}-z-\frac{1}{N} \sum_{\alpha=1}^{N-1} \frac{\xi_{\alpha}^{(k)}}{\lambda_{\alpha}^{(k)}-z}\right]^{-1}
$$

где

$$
\xi_{\alpha}^{(k)}:=N\left|\mathbf{a}^{(k)} \cdot \mathbf{u}_{\alpha}^{(k)}\right|^{2},
$$


a $\mathbf{a}^{(k)}$ есть $k$-й столбец матрицы $H$ без диагонального элемента $h_{k k}$. Взяв мнимую часть и использовав неравенство

$$
\mathscr{N}_{I} \leqslant C N \eta \operatorname{Im} m(z), \quad z=E+i \eta,
$$

мы получаем

$$
\mathscr{N}_{I} \leqslant C N \eta^{2} \sum_{k=1}^{N}\left|\sum_{\alpha: \lambda_{\alpha}^{(k)} \in I} \xi_{\alpha}^{(k)}\right|^{-1} .
$$

Имеется элементарный факт, что для любого фиксированного $k$ собственные значения $\lambda_{1} \leqslant \cdots \leqslant \lambda_{N}$ матрицы $H$ и собственные значения $\mu_{1} \leqslant \cdots \leqslant \mu_{N-1}$ матрицы $H^{(k)}$ чередуются, т. е.

$$
\lambda_{1} \leqslant \mu_{1} \leqslant \lambda_{2} \leqslant \mu_{2} \leqslant \cdots \leqslant \mu_{N-1} \leqslant \lambda_{N}
$$

В этом можно убедиться, анализируя соотношения, выражающие собственные значения $\lambda$ через собственные значения $\mu$ :

$$
\lambda-h_{i i}=\sum_{\alpha=1}^{N-1} \frac{\left|\mathbf{a}^{i} \cdot \mathbf{u}_{\alpha}\right|^{2}}{\lambda-\mu_{\alpha}},
$$

где $\mathbf{u}_{\alpha}$ - нормированный собственный вектор минора, принадлежащий собственному значению $\mu_{\alpha}$.

Из свойства чередования сразу вытекает, что в отрезке $I$ лежит по крайней мере $\mathscr{N}_{I}-1$ собственных значений $\lambda_{\alpha}^{(k)}$. Для любого фиксированного $k$ случайные величины $\left\{\xi_{\alpha}^{(k)}: \alpha=1, \ldots, N-1\right\}$ являются почти независимыми и имеют математическое ожидание 1. Следовательно, вероятность события

$$
\Omega_{k}:=\left\{\sum_{\alpha: \lambda_{\alpha}^{(k)} \in I} \xi_{\alpha}^{(k)} \leqslant \delta\left(\mathscr{N}_{I}-1\right)\right\}
$$

пренебрежимо мала при малых $\delta$ [57; лемма 4.7]. Тогда из (1.88) мы получаем, что на дополнении ко всем событиям $\Omega_{k}$ имеет место неравенство

$$
\mathscr{N}_{I} \leqslant \frac{C N^{2} \eta^{2}}{\delta\left(\mathscr{N}_{I}-1\right)},
$$

из которого следует, что $\mathscr{N}_{I} \leqslant C N \eta$ с очень большой вероятностью. Одним из точных результатов такого типа является следующая лемма.

ЛЕмма 1.10 [57; теорема 4.6]. Предположим, что распределение индивидуальных элементов $\nu$ гауссово убывает. Тогда для любого отрезка I длинъ $|I| \geqslant(\log N) / N$ миц имеем

$$
\mathrm{P}\left(\mathscr{N}_{I} \geqslant K N|I|\right) \leqslant C e^{-c \sqrt{K N|I|}} .
$$

Заметим, что условие гауссова убывания может быть ослаблено. Кроме того, несколько более слабый результат имеет место даже для более коротких отрезков $|I| \geqslant 1 / N$ (см. теорему 5.1 в [57]). 
Доказательство теоремы 1.9 также начинается с соотношений (1.85) и (1.87). Из них вытекает неравенство

$$
\mathscr{N}_{I} \leqslant C \eta \sum_{k=1}^{N} \frac{1}{\left(a_{k}^{2}+b_{k}^{2}\right)^{1 / 2}}
$$

где

$$
a_{k}:=\eta+\frac{1}{N} \sum_{\alpha=1}^{N-1} \frac{\eta \xi_{\alpha}^{(k)}}{\left(\lambda_{\alpha}^{(k)}-E\right)^{2}+\eta^{2}}, \quad b_{k}:=h_{k k}-E-\frac{1}{N} \sum_{\alpha=1}^{N-1} \frac{\left(\lambda_{\alpha}^{(k)}-E\right) \xi_{\alpha}^{(k)}}{\left(\lambda_{\alpha}^{(k)}-E\right)^{2}+\eta^{2}},
$$

т. е. $a_{k}$ и $b_{k}$ являются соответственно мнимой и вещественной частью обратных к слагаемым в (1.85). Доказательство леммы 1.10 использовало только мнимую часть, т. е. $b_{k}$ в (1.90) не принималось во внимание в оценке (1.88). Однако в доказательстве теоремы 1.9 мы также существенно использовали $b_{k}$. Так как для типичных значений выполнено неравенство $1 / N \lesssim\left|\lambda_{\alpha}^{(k)}-E\right|$, мы видим, что величина $a_{k}^{2}$ намного меньше, чем $b_{k}^{2}$, если $\eta \ll 1 / N$, что соответствует режиму, в котором имеет место оценка Вегнера и отталкивание уровней.

При некоторых предположениях гладкости распределения отдельных элементов $\mathrm{d} \nu$ распределение величин $\xi_{\alpha}^{(k)}$ также будет гладким, даже если мы зафиксируем индекс $k$ и наложим условие на минор $H^{(k)}$, т. е. если мы зафиксируем собственные значения $\lambda_{\alpha}^{(k)}$ и собственные векторы $\mathbf{u}_{\alpha}^{(k)}$. Несмотря на то что случайные величины $\xi_{\alpha}^{(k)}=N\left|\mathbf{a}^{(k)} \cdot \mathbf{u}_{\alpha}^{(k)}\right|^{2}$ не являются независимыми при различных $\alpha$, они достаточно некоррелированы, так что распределение величины $b_{k}$ наследует некоторые свойства гладкости распределения $\mathbf{a}^{(k)}$. Наличие достаточной гладкости распределения величины $b_{k}$ влечет конечность математического ожидания $\left(a_{k}^{2}+b_{k}^{2}\right)^{-p / 2}$ для любого $p>0$. Это даст нам оценку на $p$-й момент для $\mathscr{N}_{I}$, из которой будет вытекать неравенство (1.84).

Мы изложим это рассуждение для эрмитовых матриц в наиболее простом случае $n=1$. Из (1.90) мы получаем

$$
\mathrm{P}\left(\mathscr{N}_{I} \geqslant 1\right) \leqslant \mathrm{E} \mathscr{N}_{I}^{2} \leqslant C(N \eta)^{2} \mathrm{E} \frac{1}{a_{1}^{2}+b_{1}^{2}} .
$$

Опуская индекс $k=1$ и вводя обозначения

$$
d_{\alpha}=\frac{N\left(\lambda_{\alpha}-E\right)}{N^{2}\left(\lambda_{\alpha}-E\right)^{2}+\varepsilon^{2}}, \quad c_{\alpha}=\frac{\varepsilon}{N^{2}\left(\lambda_{\alpha}-E\right)^{2}+\varepsilon^{2}},
$$

мы получаем

$$
\mathrm{P}\left(\mathscr{N}_{I} \geqslant 1\right) \leqslant C \varepsilon^{2} \mathrm{E}\left[\left(\sum_{\alpha=1}^{N-1} c_{\alpha} \xi_{\alpha}\right)^{2}+\left(h-E-\sum_{\alpha=1}^{N-1} d_{\alpha} \xi_{\alpha}\right)^{2}\right]^{-1} .
$$

Как показывает одна из версий локального полукругового закона (см. теорему 1.11 ниже), на расстоянии $O(1 / N)$ от $E$ с очень большой вероятностью найдутся несколько собственных значений $\lambda_{\alpha}$. Выбрав четыре таких собственных значения, мы можем гарантировать, что для некоторого индекса $\gamma$ имеют место неравенства

$$
c_{\gamma}, c_{\gamma+1} \geqslant C \varepsilon, \quad d_{\gamma+2}, d_{\gamma+3} \geqslant C
$$


с некоторой положительной константой $C$. Если бы величины $\xi_{\alpha}$ были независимы и распределены как квадрат комплексной случайной величины $z_{\alpha}$ с гладкой убывающей плотностью $\mathrm{d} \mu(z)$ на комплексной плоскости, то математическое ожидание в (1.91) оценивалось бы сверху следующей величиной:

$$
\sup _{E} \int \frac{1}{\left(c_{\gamma}\left|z_{\gamma}\right|^{2}+c_{\gamma+1}\left|z_{\gamma+1}\right|^{2}\right)^{2}+\left(E-d_{\gamma+2}\left|z_{\gamma+2}\right|^{2}-d_{\gamma+3}\left|z_{\gamma+3}\right|^{2}\right)^{2}} \prod_{j=0}^{3} \mathrm{~d} \mu\left(z_{\gamma+j}\right) .
$$

Простое вычисление показывает, что этот интеграл оценивается сверху величиной $C \varepsilon^{-1}$, при условии выполнения нижних оценок (1.92). Применяя эту оценку совместно с (1.91), мы получаем (1.84) при $n=1$. Доказательство для произвольного $n$ проводится по индукции. Разница между эрмитовым и симметрическим случаями проявляется в том, что величины $\xi_{\alpha}$ являются квадратами комплексных и вещественных величин соответственно. Это дает различные оценки интегралов вида (1.93), что приводит к разным показателям в (1.84).

В этом доказательстве мы использовали следующий вариант локального полукругового закона.

Теорема 1.11 [57; теорема 3.1]. Пусть $H$ - эрмитова или симметрическая $(N \times N)$-матрица Вигнера, распределение отдельных элементов которой имеет гауссово убъвание. Рассмотрим $\kappa>0$ и зафиксируем энергию $E \in[-2+\kappa, 2-\kappa]$. Тогда существуют положительные константы, зависящие только от к, и универсальная константа $c_{1}>0$, для которых имеют место следующие утверждения.

1) Для любого $\delta \leqslant c_{1} \kappa$ и $N \geqslant 2$ мы имеем:

$$
\mathrm{P}\left(\left|m(E+i \eta)-m_{\mathrm{sc}}(E+i \eta)\right| \geqslant \delta\right) \leqslant C e^{-c \delta \sqrt{N \eta}},
$$

при выполнении условия $K /(N \sqrt{E}) \leqslant \eta \leqslant 1$, где $K$ - большая универсальная константа.

2) Пусть $\mathscr{N}_{\eta^{*}}(E)=\mathscr{N}_{I^{*}}$ обозначает число собственных значений на отрезке $I^{*}:=\left[E-\eta^{*} / 2, E+\eta^{*} / 2\right]$. Тогда для любого $\delta \leqslant c_{1} \kappa$ найдется константа $K_{\delta}$, зависящая только от $\delta$, для которой неравенство

$$
\mathrm{P}\left\{\left|\frac{\mathscr{N}_{\eta^{*}}(E)}{N \eta^{*}}-\varrho_{\mathrm{sc}}(E)\right| \geqslant \delta\right\} \leqslant C e^{-c \delta^{2} \sqrt{N \eta^{*}}}
$$

выполнено для всех $\eta^{*}$, удовлетворяющих условию $K_{\delta} / N \leqslant \eta^{*} \leqslant 1$, для всех $N \geqslant 2$.

\section{2. Локальный полукруговой закон и делокализация}

Каждый подход к доказательству универсальности для обобщенных матриц Вигнера требует начального анализа локальной плотности собственных значений. Полукруговой закон Вигнера [21] (и его аналог для матриц Уишарта закон Марченко-Пастура [19]) исторически были одними из первых результатов, полученных для случайных матриц. При этом, как правило, показывается, 
что эмпирическая плотность слабо сходится в макроскопическом масштабе, т. е. на отрезках, содержащих $O(N)$ собственных значений. Основываясь на наших результатах [55]-[57], [89], [7]-[9], здесь мы показываем, что полукруговой закон справедлив также и при значительно меньших порядках величин. В п. 2.2 мы используем формализм [57], в то время, как в п. 2.3 мы используем результаты работ [9], [8]. Первый формализм направлен на исследование преобразования Стилтьеса или следа резольвенты. Второй формализм разработан для получения полукругового закона для индивидуальных диагональных элементов резольвенты, и он также дает оценку на внедиагональные элементы. Наиболее сильный результат [7], который имеет место равномерно по параметру энергии, изложен в п. 2.4. Наконец, в п. 2.5 мы показываем, как вывести свойство делокализации собственных векторов из локального полукругового закона.

2.1. Формулы для резольвент. Для определенности мы дадим доказательство в эрмитовом случае, но все дальнейшие формулы с очевидными изменениями справедливы и для других классов симметрии. Вначале мы приведем несколько полезных формул для резольвент. Их доказательства получаются элементарными рассуждениями из линейной алгебры.

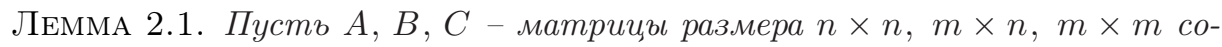
ответственно. Определим $(m+n) \times(m+n)$-матрицу $D$ бормулой

$$
D:=\left(\begin{array}{cc}
A & B^{*} \\
B & C
\end{array}\right)
$$

$u(n \times n)$-матрицу $\widehat{D}$ формулой

$$
\widehat{D}:=A-B^{*} C^{-1} B .
$$

Тогда при любых $1 \leqslant i, j \leqslant n$ мы имеем соотношение

$$
\left(D^{-1}\right)_{i j}=\left(\widehat{D}^{-1}\right)_{i j}
$$

для соответствующих матричных элементов.

Напомним, что мы обозначали через $G_{i j}=G_{i j}(z)$ матричные элементы резольвенты

$$
G_{i j}=\left(\frac{1}{H-z}\right)_{i j} .
$$

Пусть $G^{(i)}$ обозначает резольвенту матрицы $H^{(i)}$, которая представляет собой $(N-1) \times(N-1)$-минор матрицы $H$, получаемый удалением $i$-х строки и столбца. Пусть $\mathbf{a}^{i}=\left(h_{1 i}, h_{2 i}, \ldots, h_{N i}\right)^{t}-i$-й столбец матрицы $H$, из которого иногда удалены один или более элементов. Мы всегда будем сохранять исходную нумерацию строк и столбцов, так что это не будет приводить к недоразумениям: если столбец $\mathbf{a}^{i}$ умножается на матрицу, у которой удалены $j$-е столбец и строка, то мы также удаляем $j$-й элемент из $\mathbf{a}^{i}$. Используя аналогичные соглашения, мы можем определить матрицы $G^{(i j)}$ и т. д. Верхние индексы в скобках для резольвент всегда будут означать "после удаления соответствующих столбца и строки". В частности, из независимости матричных элементов вытекает, 
что матрица $G^{(i j)}$, например, не зависит от $i$-й и $j$-й строк и $i$-го и $j$-го столбцов матрицы $H$. Это будет полезно при разведении зависимостей в формулах.

Используя лемму 2.1 для $n=1, m=N-1$, мы получаем

$$
G_{i i}=\frac{1}{h_{i i}-z-\mathbf{a}^{i} \cdot \frac{1}{H^{(i)}-z} \mathbf{a}^{i}}=\frac{1}{h_{i i}-z-\mathbf{a}^{i} \cdot G^{(i)} \mathbf{a}^{i}},
$$

где $\mathbf{a}^{i}$ есть $i$-й столбец, из которого удален $i$-й элемент $h_{i i}$.

Для элементов вне диагонали необходимо произвести разложение по двум строкам. В этом случае, пусть $\mathbf{a}^{1}$ и $\mathbf{a}^{2}$ обозначают первый и второй столбцы матрицы $H$ с удаленными первыми и вторыми элементами, т.е. из первого столбца удаляются $h_{11}$ и $h_{21}$, а из второго - $h_{12}$ и $h_{22}$. Применим лемму 2.1 для $n=2, m=N-2$ и матриц $D=H-z, B=\left[\mathbf{a}^{1}, \mathbf{a}^{2}\right], C=H^{12}-z$. Тогда мы можем вычислить матрицу $\widehat{D}$, которую в этом случае мы будем называть $K^{(12)}$ :

$$
\widehat{D}=\left(\begin{array}{cc}
h_{11}-z-\mathbf{a}^{1} \cdot G^{(12)} \mathbf{a}^{1} & h_{12}-\mathbf{a}^{1} \cdot G^{(12)} \mathbf{a}^{2} \\
h_{21}-\mathbf{a}^{2} \cdot G^{(12)} \mathbf{a}^{1} & h_{22}-z-\mathbf{a}^{2} \cdot G^{(12)} \mathbf{a}^{2}
\end{array}\right)=:\left(\begin{array}{cc}
K_{11}^{(12)} & K_{12}^{(12)} \\
K_{21}^{(12)} & K_{22}^{(12)}
\end{array}\right)
$$

где мы для удобства ввели обозначения

$$
K_{i j}^{(12)}:=h_{i j}-z \delta_{i j}-\mathbf{a}^{i} \cdot G^{(12)} \mathbf{a}^{j}, \quad i, j=1,2 .
$$

Из леммы 2.1 мы получаем, например, следующие соотношения:

$$
\begin{aligned}
G_{11} & =\frac{K_{22}^{(12)}}{K_{22}^{(12)} K_{11}^{(12)}-K_{12}^{(12)} K_{21}^{(12)}}, \\
G_{12} & =-\frac{K_{12}^{(12)}}{K_{22}^{(12)} K_{11}^{(12)}-K_{12}^{(12)} K_{21}^{(12)}}=-G_{22} \frac{K_{12}^{(12)}}{K_{11}^{(12)}}=-G_{22} G_{11}^{(2)} K_{12}^{(12)} .
\end{aligned}
$$

На последнем шаге мы использовали тождество

$$
G_{11}^{(2)}=\frac{1}{K_{11}^{(12)}}
$$

которое есть в точности разложение по одной строке (2.4), примененное к минору $H^{(2)}$ матрицы $H$, получаемому удалением второй строки и второго столбца.

Имеется другой набор формул, который позволяет сравнивать резольвенты матриц $H$ и $H^{(1)}$. Например, для любых $i \neq j$ имеем

$$
G_{i i}=G_{i i}^{(j)}+\frac{G_{i j} G_{j i}}{G_{j j}}
$$

Это соотношение легко проверить на матрицах размера $(2 \times 2)$ и их обратных:

$$
M=\left(\begin{array}{ll}
a & b \\
c & d
\end{array}\right), \quad M^{-1}=\frac{1}{\Delta}\left(\begin{array}{cc}
d & -c \\
-b & a
\end{array}\right), \quad \text { где } \Delta=a d-b c,
$$


так что проверка соотношения (2.10), например, при $i=1$ и $j=2$ сводится к тождеству

$$
\frac{d}{\Delta}=\frac{1}{a}+\frac{\frac{c}{\Delta} \frac{b}{\Delta}}{\frac{a}{\Delta}}
$$

Для бо́льших матриц нужно лишь применить соотношение (2.3). Заметим, что при помощи формул $(2.7),(2.8)$ и (2.9) мы уже выразили все резольвенты, появляющиеся в $(2.10)$, в терминах элементов $(2 \times 2)$-матриц $K^{(12)}$ из $(2.5)$, которые можно использовать в качестве матриц $M$ выше. Аналогично, для любых трех различных индексов $i, j, k$ мы имеем

$$
G_{i j}=G_{i j}^{(k)}+\frac{G_{i k} G_{k j}}{G_{k k}} .
$$

Это тождество можно проверить для матриц размера $3 \times 3$, а затем доказать по индукции в общем случае.

2.2. Полукруговой закон при помощи резольвент: набросок грубого метода. В этом пункте мы дадим набросок доказательства следующей теоремы.

Tеорема 2.2. Пусть $z=E+i \eta, 1 / N \ll \eta \ll 1 u \kappa:=|| E|-2|$. Pacсмотрим матрицу Вигнера $H$ с резольвентой $G(z)=(H-z)^{-1}$ и положим $m(z):=\frac{1}{N} \operatorname{Tr} G(z)$. Предположим, что распределение отдельных элементов $\nu$ имеет гауссово убывание $(\vartheta=2$ в (1.67)). Тогда имеет место следующее приближение:

$$
\left|m(z)-m_{\mathrm{sc}}(z)\right| \leqslant \min \left\{\frac{(\log N)^{C}}{\sqrt{N \eta \kappa}}, \frac{(\log N)^{C}}{(N \eta)^{1 / 4}}\right\}
$$

с очень большой вероятностью.

Локальный полукруговой закон был доказан в этом виде в [6; предложение 8.1] для выборочных ковариационных матриц (с заменой распределения полукруга распределением Марченко-Пастура), но то же самое (и даже более простое) доказательство проходит и для матриц Вигнера. Исходное доказательство было дано при условии гауссова убывания, но это условие может легко быть ослаблено до субэкспоненциального убывания, так как оно влияет только на оценку вероятности нарушения события (2.12). [Для специалистов: в наших предыдущих работах, вплоть до [6], мы, как правило, использовали теорему Хансона-Райта [101] для оценки вероятностей больших уклонений квадратичных форм. Это дает очень хороший контроль над хвостами, но требует гауссова убывания. В наших недавних работах мы использовали лемму 2.12, основанную на мартингальных неравенствах и требующую лишь субэкспоненциального убывания, которое в действительности может быть ослаблено до полиномиального, но оценки хвостовых вероятностей здесь слабее.]

Переходя к доказательству, мы начнем с соотношения (2.4) и представим $G_{i i}$ в виде

$$
G_{i i}=\frac{1}{h_{i i}-z-\mathrm{E}_{i} \mathbf{a}^{i} \cdot G^{(i)} \mathbf{a}^{i}-Z_{i}},
$$


где мы разделили величины

$$
\mathbf{a}^{i} \cdot G^{(i)} \mathbf{a}^{i}=\mathrm{E}_{i} \mathbf{a}^{i} \cdot G^{(i)} \mathbf{a}^{i}+Z_{i}, \quad Z_{i}:=\mathbf{a}^{i} \cdot G^{(i)} \mathbf{a}^{i}-\mathrm{E}_{i} \mathbf{a}^{i} \cdot G^{(i)} \mathbf{a}^{i}
$$

на их математические ожидания и флуктуации; здесь $\mathrm{E}_{i}$ обозначает математическое ожидание по отношению к переменным в $i$-й строке и $i$-м столбце. В частности, величина $G^{(i)}$ не зависит от $\mathbf{a}^{i}$, так что нам необходимо подсчитать математические ожидания и флуктуации квадратичных функций.

Математическое ожидание вычисляется легко:

$$
\mathrm{E}_{i} \mathbf{a}^{i} \cdot G^{(i)} \mathbf{a}^{i}=\mathrm{E}_{i} \sum_{k, l \neq i} \overline{\mathbf{a}_{k}^{i}} G_{k l}^{(i)} \mathbf{a}_{l}^{i}=\sum_{k, l \neq i} \mathrm{E}_{i} \bar{h}_{i k} G_{k l}^{(i)} h_{i l}=\frac{1}{N} \sum_{k \neq i} G_{k k}^{(i)},
$$

где на последнем шаге мы использовали независимость различных матричных элементов, т. е. равенство $\mathrm{E}_{i} \bar{h}_{i k} h_{i l}=\frac{1}{N} \delta_{k l}$. Суммирование всегда производится по всем индексам от 1 до $N$, кроме тех индексов, которые явно исключены.

Аналогично тому, как мы определяли

$$
m(z)=\frac{1}{N} \operatorname{Tr} G(z)=\frac{1}{N} \sum_{k=1}^{N} G_{k k}(z),
$$

определим

$$
m^{(i)}(z):=\frac{1}{N-1} \operatorname{Tr} G^{(i)}(z)=\frac{1}{N-1} \sum_{k \neq i} G_{k k}^{(i)}(z) .
$$

Следующая лемма позволяет сравнивать следы матриц $G$ и $G^{(i)}$.

Лемма 2.3. Для любого $1 \leqslant i \leqslant N$ имеют место неравенства

$$
\left|m(z)-m^{(i)}(z)\right| \leqslant \frac{C}{N \eta}, \quad \eta=\operatorname{Im} z>0 .
$$

ДокАЗАТЕЛЬство. Пусть

$$
F(x):=\frac{1}{N} \#\left\{\lambda_{j} \leqslant x\right\}, \quad F^{(i)}(x):=\frac{1}{N-1} \#\left\{\mu_{j} \leqslant x\right\}
$$

обозначают нормированные функции числа собственных значений. Свойство чередования собственных значений матриц $H$ и $H^{(i)}($ см. $(1.89))$ в терминах этих функций означает, что

$$
\sup _{x}\left|N F(x)-(N-1) F^{(i)}(x)\right| \leqslant 1 .
$$

Тогда, интегрируя по частям, мы получаем

$$
\begin{aligned}
\mid m(z) & -\left(1-\frac{1}{N}\right) m^{(i)}(z)|=| \int \frac{\mathrm{d} F(x)}{x-z}-\left(1-\frac{1}{N}\right) \int \frac{\mathrm{d} F^{(i)}(x)}{x-z} \mid \\
= & \frac{1}{N}\left|\int \frac{N F(x)-(N-1) F^{(i)}(x)}{(x-z)^{2}} \mathrm{~d} x\right| \leqslant \frac{1}{N} \int \frac{\mathrm{d} x}{|x-z|^{2}} \leqslant \frac{C}{N \eta},
\end{aligned}
$$

что вместе с тривиальной оценкой $\left|m^{(i)}\right| \leqslant \eta^{-1}$ доказывает неравенство (2.14). 
Возвращаясь к (2.13), мы, таким образом, имеем

$$
G_{i i}=\frac{1}{-z-m(z)+\Omega_{i}}
$$

где

$$
\Omega_{i}:=h_{i i}-Z_{i}+O\left(\frac{1}{N \eta}\right) .
$$

Суммируя матричные элементы (2.16), мы получаем

$$
m=\frac{1}{N} \sum_{i=1}^{N} \frac{1}{-z-m(z)+\Omega_{i}} .
$$

Предположим, что величина $\Omega:=\max _{i}\left|\Omega_{i}\right|$ мала и $|z+m| \geqslant C>0$, т. е. возможно разложение

$$
\frac{1}{-z-m(z)+\Omega_{i}}=\frac{1}{-z-m(z)}+O(\Omega) .
$$

Тогда мы имеем следующее самосогласованное уравнение на $m$ :

$$
m+\frac{1}{z+m}=O(\Omega)
$$

Напомним, что преобразование Стилтьеса полукругового закона

$$
m_{\mathrm{sc}}(z)=\int_{\mathbb{R}} \frac{\varrho_{\mathrm{sc}}(x) \mathrm{d} x}{x-z}
$$

может быть охарактеризовано как единственное решение квадратного уравнения

$$
m_{\mathrm{sc}}(z)+\frac{1}{z+m_{\mathrm{sc}}(z)}=0
$$

удовлетворяющее условию $\operatorname{Im} m_{\mathrm{sc}}(z)>0$ при $\operatorname{Im} z>0$. Следовательно, мы можем использовать стабильность уравнения (2.20) для того, чтобы определить решение $m$ уравнения (2.19). Стабильность ухудшается вблизи границ спектра $z \sim \pm 2$, и мы имеем следующий точный результат, который может быть доказан методами элементарного анализа.

Лемма 2.4 [6; лемма 8.4]. Зафиксируем $z=E+i \eta, \eta>0$ и положим $\kappa:=|| E|-2|$. Предположим, что функиия $m=m(z)$ имеет положительную мнимую часть и что

$$
\left|m+\frac{1}{z+m}\right| \leqslant \delta
$$

Тогда

$$
\left|m-m_{\mathrm{sc}}(z)\right| \leqslant \frac{C \delta}{\sqrt{\kappa+\delta}} .
$$


Используя этот результат, мы получаем

$$
\left|m(z)-m_{\mathrm{sc}}(z)\right| \leqslant \frac{C \Omega}{\sqrt{\kappa+\Omega}} .
$$

Теперь мы можем дать грубую оценку размера $\Omega$. Очевидно, что $\left|h_{i i}\right| \lesssim$ $N^{-1 / 2}$. Если распределение отдельных элементов убывает субэкспоненциально, то мы можем гарантировать, что все диагональные элементы удовлетворяют этой оценке с очень большой вероятностью. Напомним, что условие (1.67) влечет неравенство

$$
\mathrm{P}\left(\left|h_{i i}\right| \geqslant M^{\alpha} N^{-1 / 2}\right) \leqslant C e^{-M}
$$

для всех $i$. Положив $M=(\log N)^{1+\varepsilon}$, мы получаем

$$
\mathrm{P}\left(\exists i:\left|h_{i i}\right| \geqslant(\log N)^{(1+\varepsilon) \alpha} N^{-1 / 2}\right) \leqslant C N e^{-(\log N)^{1+\varepsilon}} \leqslant C e^{-(\log N)^{1+\varepsilon}},
$$

что убывает быстрее, чем любой многочлен.

Чтобы оценить величину $Z_{i}$, мы вычислим ее второй момент

$$
\mathrm{E}\left|Z_{i}\right|^{2}=\sum_{k, l \neq i} \sum_{k^{\prime}, l^{\prime} \neq i} \mathrm{E}_{i}\left(\left[\bar{h}_{i k} G_{k l}^{(i)} h_{i l}-\mathrm{E}_{i} \bar{h}_{i k} G_{k l}^{(i)} h_{i l}\right]\left[h_{i k^{\prime}} \bar{G}_{k^{\prime} l^{\prime}}^{(i)} \bar{h}_{i l^{\prime}}-\mathrm{E}_{i} h_{i k^{\prime}} \bar{G}_{k^{\prime} l^{\prime}}^{(i)} \bar{h}_{i l^{\prime}}\right]\right)
$$

Так как $\mathrm{E} h=0$, то ненулевой вклад в эту сумму дают только соответствующие (спаренные) комбинации индексов у $h$ и $\bar{h}$. Предположим для простоты, что $\mathrm{E} h^{2}=0$; этого всегда можно достичь, например, в случае, когда распределения действительной и мнимой частей совпадают. Тогда множители $h$ в предыдущей формуле должны спариваться таким образом, что $h_{i k}=h_{i k^{\prime}}$ и $h_{i l}=h_{i l^{\prime}}$, т. е. $k=k^{\prime}$ и $l=l^{\prime}$. Заметим, что спаривание $h_{i k}=h_{i l}$ будет давать нуль, так как математическое ожидание вычитается из выражений. В результате получим

$$
\mathrm{E}\left|Z_{i}\right|^{2}=\frac{1}{N^{2}} \sum_{k, l \neq i}\left|G_{k l}^{(i)}\right|^{2}+\frac{m_{4}-1}{N^{2}} \sum_{k \neq i}\left|G_{k k}^{(i)}\right|^{2}
$$

где $m_{4}=\mathrm{E}|\sqrt{N} h|^{4}$ - четвертый момент распределения отдельных собственных значений. Первый член в сумме может быть вычислен следующим образом:

$$
\frac{1}{N^{2}} \sum_{k, l \neq i}\left|G_{k l}^{(i)}\right|^{2}=\frac{1}{N^{2}} \sum_{k \neq i}\left(\left|G^{(i)}\right|^{2}\right)_{k k}=\frac{1}{N \eta} \frac{1}{N} \sum_{k} \operatorname{Im} G_{k k}^{(i)}=\frac{1}{N \eta} \operatorname{Im} m^{(i)},
$$

где $|G|^{2}=G G^{*}$ и мы воспользовались соотношением

$$
|G|^{2}=\frac{1}{|H-E|^{2}+\eta^{2}}=\frac{1}{\eta} \operatorname{Im} G
$$

Для того чтобы оценить эту величину, нам понадобится верхняя оценка локальной плотности в масштабе $\eta \gg 1 / N$. Для любого отрезка $I \subset \mathbb{R}$ пусть $\mathscr{N}_{I}:=\#\left\{\lambda_{j} \in I\right\}$ обозначает число собственных значений в $I$. Лемма 1.10 из п. 1.7 показывает, что неравенство $\mathscr{N}_{I} \lesssim N|I|$ имеет место с очень большой вероятностью. Применив эту лемму к матрице $H^{(i)}$ с собственными значениями 
$\mu_{1}, \mu_{2}, \ldots, \mu_{N-1}$, мы получаем

$$
\operatorname{Im} m^{(i)}=\frac{1}{N-1} \sum_{\alpha} \frac{\eta}{\left(E-\mu_{\alpha}\right)^{2}+\eta^{2}} \lesssim \int \frac{\eta}{(x-E)^{2}+\eta^{2}} \varrho_{\mathrm{sc}}(x) \mathrm{d} x \lesssim O(1) .
$$

Напомним, что символ $\lesssim$ здесь обозначает “с точностью до множителей порядка $\log N^{\prime \prime}$.

Второй член в формуле (2.24) мы можем ограничить сверху, воспользовавшись тривиальной оценкой $\left|G_{k k}^{(i)}\right| \leqslant \eta^{-1}$, которая дает

$$
\begin{aligned}
\frac{1}{N^{2}} \sum_{k \neq i}\left|G_{k k}^{(i)}\right|^{2} & \leqslant \frac{1}{N^{2} \eta} \sum_{k \neq i}\left|G_{k k}^{(i)}\right| \leqslant \frac{1}{N^{2} \eta} \sum_{k \neq i} \sum_{\alpha=1}^{N-1} \frac{\left|\mathbf{u}_{\alpha}(k)\right|^{2}}{\left|\lambda_{\alpha}-z\right|} \\
& \leqslant \frac{1}{N \eta} \frac{1}{N} \sum_{\alpha=1}^{N-1} \frac{1}{\left|\lambda_{\alpha}-z\right|} \lesssim \frac{1}{N \eta}
\end{aligned}
$$

где $\mathbf{u}_{\alpha}-$ (нормированный) собственный вектор для $\mu_{\alpha}$, а на последнем шаге мы воспользовались оценкой, аналогичной $(2.26)$.

Оценки (2.25) и (2.27) подтверждают, что величина $Z_{i}$ имеет размер примерно

$$
\left|Z_{i}\right| \lesssim \frac{1}{\sqrt{N \eta}}
$$

по крайней мере в смысле второго момента. Можно также вычислить более высокие моменты или даже получить еще более сильные результаты о концентрации (например, при условии выполнения логарифмического неравенства Соболева) для улучшения оценки (2.28) таким образом, что она будет иметь место в смысле вероятности.

Из формул $(2.17),(2.22)$ и (2.28) мы получаем

$$
\Omega \lesssim \frac{C}{\sqrt{N}}+\frac{C}{\sqrt{N \eta}}+\frac{C}{N \eta}
$$

Так как нас интересует порядок величин $1 / N \ll \eta \leqslant 1$, то мы имеем $\Omega \lesssim$ $(N \eta)^{-1 / 2}$. Применяя это совместно с оценкой стабильности $(2.21)$, получаем

$$
\left|m(z)-m_{\mathrm{sc}}(z)\right| \lesssim \min \left\{\frac{1}{\sqrt{N \eta \kappa}}, \frac{1}{(N \eta)^{1 / 4}}\right\}
$$

что доказывает лемму 2.3 .

Замечательной особенностью этого метода является то, что он работает вплоть до порядков величин $\eta \sim 1 / N$. Множитель $\kappa$ выражает тот факт, что оценка ухудшается на границах. Показатели не являются оптимальными, разность $m-m_{\mathrm{sc}}$ может быть сравнена с точностью $(N \eta)^{-1}$. Это мы обсудим в следующем пункте. Улучшения можно достичь, исходя из того факта, что главный поправочный член $Z_{i}$ в (2.17) флуктуирует, и можно показать [9], что его вклады в разность $m-m_{\mathrm{sc}}$ сокращаются в главном члене. Таким образом, этот член имеет размер меньший, чем размер $Z_{i}$, предсказываемый 
вычислением дисперсии (этот эффект был впервые использован в теореме 4.1 из [89], а затем был существенно улучшен в [9] и [8]).

Подчеркнем, что наше изложение было весьма поверхностным, многие технические подробности были опущены.

\section{3. Полукруговой закон из резольвент: улучшенный метод.}

2.3.1. Формулировка теоремы и следствия. В этом пункте мы изложим улучшенный метод, позволяющий оценить матричные элементы резольвенты и также применимый к универсальным матрицам Вигнера (см. определение 1.1). Это метод является ключевой составляющей для улучшения точности полукругового закона как в терминах $(N \eta)$-степеней, так и в терминах поведения на границах. Основная часть метода состоит в анализе самосогласованного уравнения для вектора $\left(G_{11}, \ldots, G_{N N}\right)$ диагональных элементов резольвенты, а не только их суммы, которая приводила к соотношению (2.18). Как и ранее, для определенности мы формулируем результат только в случае обобщенных эрмитовых матриц Вигнера; перенос на случай симметрических матриц производится непосредственно.

Ключевым объектом будет матрица дисперсий $\Sigma$, введенная в (1.15). Напомним, что матрица $\Sigma$ является симметрической и дважды стохастической в силу (1.16), в частности, она удовлетворяет неравенствам $-1 \leqslant \Sigma \leqslant 1$. Предположим, что для спектра матрицы $\Sigma$ справедливо включение

$$
\operatorname{Spec}(\Sigma) \subset\left[-1+\delta_{-}, 1-\delta_{+}\right] \cup\{1\}
$$

где $\delta_{ \pm}-$некоторая неотрицательная константа. Мы всегда будем предполагать, что выполнено следующее условие на спектр:

\section{1 является простым собственным значением матрицы $\Sigma$ и}

$\delta_{-}$- положительная константа, не зависящая от $N$.

Для матриц Вигнера все элементы матрицы $\Sigma$ одинаковы и $\delta_{ \pm}=1$. Легко доказать (см. лемму А.1 в [9]), что условие (2.30) выполнено для случайных ленточных матрии, (см. определение в (1.18)), с $\delta_{-}>0$, зависящим только от $f$. Для обобщенных матрии, Вигнера, т. е. для матриц Вигнера со сравнимыми дисперсиями (см. определение в (1.17)), легко проверить, что имеет место неравенство

$$
\delta_{ \pm} \geqslant C_{\text {inf }}>0 \text {. }
$$

Тот факт, что $\delta_{+}>0$ для обобщенных матриц Вигнера, позволяет лучше контролировать поведение оценок на границах. В этом заключается основная причина того, что формулировки нижеследующего утверждения отличаются для универсальных матриц Вигнера (см. (1.1)) и для обобщенных матриц Вигнеpa (1.17).

Точность локального полукругового закона зависит от трех факторов. Первым из них является разрешение (масштаб, в котором имеет место полукруговой закон), оно задается мнимой частью $\eta=\operatorname{Im} z$ спектрального параметра $z$ в преобразовании Стилтьеса. Вторым фактором является расстояние до границ спектра, которое измеряется параметром $\kappa=|| E|-2|$. Последним фактором является размер типичных матричных элементов, который измеряется 
величиной

$$
M:=\frac{1}{\max _{i j} \sigma_{i j}^{2}},
$$

называемой шириной матрицы. Например, для матриц Вигнера мы имеем $M=N$, для обобщенных матриц Вигнера (1.1) имеем $M \sim N$, а для случайных ленточных матриц (1.18) имеем $M \sim W$.

ТЕОРЕма 2.5 (локальный полукруговой закон для универсальных матриц Вигнера, [8; теорема 2.1]). Пусть $H$ - эрмитова случайная $(N \times N)$-матрица $c \mathrm{E} h_{i j}=0,1 \leqslant i, j \leqslant N$, и дисперсиями $\sigma_{i j}^{2}$ удовлетворяющими условиям (1.16) и (2.30). Предположим далее, что распределения матричных элементов имеют равномерное субэкспоненииальное убывание в том смысле, что существуют константы $C, \vartheta>0$, не зависящие от $N$, такие, что для любого $x>0$ и для каждой пары $(i, j)$ мы имеем

$$
\mathrm{P}\left(\left|h_{i j}\right| \geqslant x\left|\sigma_{i j}\right|\right) \leqslant C \exp \left(-x^{\vartheta}\right) .
$$

Мъ будем параллельно рассматривать универсальные матрицы Вигнера и их специальный подкласс - обобщенные матрицы Вигнера. Эти два класса будут различатъся по значению параметра $A$ : мы положим $A=2$ для универсальньх матрии, Вигнера и $A=1$ для обобщенных матрии, Вигнера; в последнем случае результаты будут сильнее.

Рассмотрим следующую область в $\mathbb{C}$ :

$$
D:=\left\{z=E+i \eta \in \mathbb{C}:|E| \leqslant 5,0<\eta<10, \sqrt{M \eta} \geqslant(\log N)^{C_{1}}(\kappa+\eta)^{1 / 4-A}\right\},
$$

где $\kappa:=|| E|-2|$. Тогда существуют константы $C_{1}, C_{2}$ и с $>0$, зависящие только от $\vartheta$ и $\delta_{-}$в условии (2.30), такие, что для любых $\varepsilon>0 u K>0$ преобразование Стилтъеса распределения собственных значений матрищы $H$ удовлетворяет неравенству

$$
\mathrm{P}\left(\bigcup_{z \in D}\left\{\left|m(z)-m_{\mathrm{sc}}(z)\right| \geqslant \frac{N^{\varepsilon}}{M \eta(\kappa+\eta)^{A}}\right\}\right) \leqslant \frac{C(\varepsilon, K)}{N^{K}}
$$

для достаточно большого N. Диагональные матричные элементы функиии Грина $G_{i i}(z)=(H-z)^{-1}(i, i)$ удовлетворяют условию

$$
\mathrm{P}\left(\bigcup_{z \in D}\left\{\max _{i}\left|G_{i i}(z)-m_{\mathrm{sc}}(z)\right| \geqslant \frac{(\log N)^{C_{2}}}{\sqrt{M \eta}}(\kappa+\eta)^{1 / 4-A / 2}\right\}\right) \leqslant C N^{-c(\log \log N)},
$$

а для внедиагональных элементов мы имеем

$$
\mathrm{P}\left(\bigcup_{z \in D}\left\{\max _{i \neq j}\left|G_{i j}(z)\right| \geqslant \frac{(\log N)^{C_{2}}}{\sqrt{M \eta}}(\kappa+\eta)^{1 / 4}\right\}\right) \leqslant C N^{-c(\log \log N)}
$$

при достаточно большом $N$.

ЗАмЕчАниЕ 1 . Эти оценки максимальны по степеням $M \eta$, однако они не являются оптимальными в смысле поведения на границах спектра (степени параметра $\kappa$ ) и в смысле контроля над вероятностью. При более сильных условиях 
на убывание распределения отдельных элементов можно получить субэкспоненциальные оценки на вероятность, например, теорему 1.11 (см. теорему 3.1 в [57]). С другой стороны, условие субэкспоненциального убывания (2.32) может быть легко ослаблено, если мы не стремимся получить оценки ошибок, лучшие, чем любой степенной закон по $N$.

ЗАмЕчАниЕ 2. Что касается поведения на границах спектра, заметим, что в наших первых двух работах [55], [56] мы просто предполагали $\kappa \geqslant \kappa_{0}$ для некоторой положительной константы $\kappa_{0}$. Поведение вблизи границ спектра было сначала эффективно проанализировано в [57], а затем улучшенный результат был получен в теореме 4.1 из [89], но оценки все равно не были оптимальными. Наилучшим результатом для универсальных матриц Вигнера является теорема 2.5. Для обобщенных матриц Вигнера теорема 2.19 (доказанная в [7]) дает оптимальную оценку, равномерную по $\kappa$.

Оценки локального полукругового закона показывают, что эмпирическая функция подсчета собственных значений близка к функции подсчета собственных значений для полукругового закона (теорема 2.6) и что расположение собственных значений близко к классическому расположению в смысле квадратичных отклонений (теорема 2.7). Последний результат будет использован для проверки соотношения (1.66) или, точнее, условия III (3.9) ниже, которое будет основным средством в нашем гидродинамическом подходе к универсальности.

Для того чтобы точно сформулировать эти утверждения, обозначим через $\lambda_{1} \leqslant \cdots \leqslant \lambda_{N}$ упорядоченные собственные значения универсальной матрицы Вигнера. Мы определим нормированную эмпирическую функиию подсчета как

$$
\mathfrak{n}(E):=\frac{1}{N} \#\left\{\lambda_{j} \leqslant E\right\}
$$

и определим усредненную функиию подсчета как

$$
n(E)=\frac{1}{N} \mathrm{E} \#\left[\lambda_{j} \leqslant E\right] .
$$

Наконец, рассмотрим функцию распределения полукругового закона

$$
n_{\mathrm{sc}}(E):=\int_{-\infty}^{E} \varrho_{\mathrm{sc}}(x) \mathrm{d} x,
$$

которая очень близка к функции подсчета $\frac{1}{N} \#\left[\gamma_{j} \leqslant E\right] \approx n_{\mathrm{sc}}(E)$. Напомним, что значения $\gamma_{j}$ суть классические расположения собственных значений, задаваемые полукруговым законом (см. (1.65)).

Данные обозначения используются в следующих теоремах.

Tеорема 2.6 [8; теорема 6.3]. Пусть $A=2$ для универсальных матрии Вигнера, удовлетворяюших условиям (1.16), (2.31), (2.32), где $M \geqslant(\log N)^{24+6 \alpha}$. Для обобщенных матрии, Вигнера, удовлетворяющих условиям (1.16), (2.31), (1.17) и (2.32), мы положим $A=1$ (напомним, что в этом случае $M=N)$. Тогда для любых $\varepsilon>0$ и $K \geqslant 1$ найдется константа $C(\varepsilon, K)$ такая, что

$$
\mathrm{P}\left\{\sup _{|E| \leqslant 3}\left|\mathfrak{n}(E)-n_{\mathrm{sc}}(E)\right|\left[\kappa_{E}\right]^{A} \leqslant \frac{C N^{\varepsilon}}{M}\right\} \geqslant 1-\frac{C(\varepsilon, K)}{N^{K}},
$$


где функиии $\mathfrak{n}(E)$ и $n_{\mathrm{sc}}(E)$ задаются формулами (2.37) и (2.39), а $\kappa_{E}=|| E|-2|$.

Теорема 2.7 [8; теорема 7.1]. Пусть $H$ - обобщенная матрица Вигнера с субэкспоненииальным убыванием, т.е. выполнены условия (1.16), (2.31), (1.17) u (2.32). Пусть $\lambda_{j}$ обозначают собственные значения матриць $H$, a $\gamma_{j}-$ их полуклассические расположения, определяемые соотношением (1.65). Тогда для любого $\varepsilon<1 / 7$ и для любого $K>1$ существует константа $C_{K}$ такая, что

$$
\mathrm{P}\left\{\sum_{j=1}^{N}\left|\lambda_{j}-\gamma_{j}\right|^{2} \leqslant N^{-\varepsilon}\right\} \geqslant 1-\frac{C_{K}}{N^{K}}
$$

$u$

$$
\sum_{j=1}^{N} \mathrm{E}\left|\lambda_{j}-\gamma_{j}\right|^{2} \leqslant C N^{-\varepsilon}
$$

Эти теоремы являются следствиями локального полукругового закона - теоремы 2.5. Мы не будем приводить подробных доказательств, а лишь укажем полезную формулу, позволяющую переводить преобразование Стилтьеса в плотности. В этом контексте данная формула впервые появилась в работе [89].

ЛЕмма 2.8 [102; формула Хелффера-Сьостранда]. Пусть $f$-вещественнозначная функиия класса гладкости $C^{1}$ на $\mathbb{R}$. Пусть $\chi(y)$ - гладкая функция урезания с носителем внутри отрезка $[-1,1]$, удовлетворяющая условию $\chi(y)=1$ при $|y| \leqslant 1 / 2$ и имеющая ограниченные производные. Положим

$$
\tilde{f}(x+i y):=\left(f(x)+i y f^{\prime}(x)\right) \chi(y) .
$$

Тогда

$$
\begin{aligned}
f(\lambda) & =\frac{1}{2 \pi} \int_{\mathbb{R}^{2}} \frac{\partial_{\bar{z}} \tilde{f}(x+i y)}{\lambda-x-i y} \mathrm{~d} x \mathrm{~d} y \\
& =\frac{1}{2 \pi} \int_{\mathbb{R}^{2}} \frac{i y f^{\prime \prime}(x) \chi(y)+i\left(f(x)+i y f^{\prime}(x)\right) \chi^{\prime}(y)}{\lambda-x-i y} \mathrm{~d} x \mathrm{~d} y .
\end{aligned}
$$

Мы применим эту лемму следующим образом. Пусть $\varrho \in L^{1}(\mathbb{R})$ - вещественная функция, и пусть $m(z)$ - ее преобразование Стилтьеса

$$
m(z):=\int_{\mathbb{R}} \frac{\varrho(\lambda) \mathrm{d} \lambda}{\lambda-z} .
$$

Так как функция $f$ вещественна, мы имеем

$$
\begin{aligned}
\left|\int_{\mathbb{R}} f(\lambda) \varrho(\mathrm{d} \lambda)\right|= & \operatorname{Re} \int_{\mathbb{R}} f(\lambda) \varrho(\mathrm{d} \lambda)|=| \frac{1}{2 \pi} \operatorname{Re} \int_{\mathbb{R}^{2}} \partial_{\bar{z}} \tilde{f}(x+i y) m(x+i y) \mathrm{d} x \mathrm{~d} y \mid \\
\leqslant & \left|\frac{1}{2 \pi} \int_{\mathbb{R}^{2}} y f^{\prime \prime}(x) \chi(y) \operatorname{Im} m(x+i y) \mathrm{d} x \mathrm{~d} y\right| \\
& +C \int_{\mathbb{R}^{2}}\left(|f(x)|+|y|\left|f^{\prime}(x)\right|\right)\left|\chi^{\prime}(y)\right||m(x+i y)| \mathrm{d} x \mathrm{~d} y .
\end{aligned}
$$


Для того чтобы получить функцию подсчета, мы выберем

$$
f(\lambda)=f_{E, \eta}(\lambda)
$$

где $f_{E, \eta}$ - характеристическая функция на полуоси $(-\infty, E)$, сглаженная в масштабе $\eta$ (т. е. $f_{E, \eta}(\lambda) \equiv 1$ при $\lambda \leqslant E-\eta, f_{E, \eta}(\lambda) \equiv 0$ при $\lambda \geqslant E+\eta$ и $\left|f^{\prime}\right| \leqslant C \eta^{-1}$, $\left|f^{\prime \prime}\right| \leqslant C \eta^{-2}$ на отрезке $\left.[E-\eta, E+\eta]\right)$.

Второй член в правой части (2.43), как правило, безвреден, так как функция $\chi^{\prime}$ имеет носитель внутри множества $|y| \geqslant 1 / 2$, т. е. этот член требует информации о преобразовании Стилтьеса вдали от вещественной оси. В первом члене мы имеем лишь мнимую часть преобразования Стилтьеса, и легко видеть, что

$$
y \operatorname{Im} m(x+i y) \leqslant \int|\varrho(x)| \mathrm{d} x .
$$

Далее можно применить интегрирование по частям, заменяя вторую производную функции $f$ на ее первую производную, после чего интегрирование становится контролируемым даже при очень малых $\eta$.

Применяя эти соображения к мере $\varrho$, которая является разностью между эмпирической плотностью и плотностью $\varrho_{\mathrm{sc}}$, мы получаем контроль над величиной $\int f(\lambda) \varrho(\mathrm{d} \lambda)$ (которая в сущности является разностью функций подсчета) в терминах порядка величины $m-m_{\mathrm{sc}}$. Подробности можно найти в [89] и $[8]$.

2.3.2. Набросок доказательства полукругового закона для матричных элементов. Для простоты изложения мы не будем обращать внимание на проблему, связанную с границами спектра. Иными словами, мы будем предполагать, что функция $E=\operatorname{Re} z$ всегда удовлетворяет условию $\kappa=|| E|-2| \geqslant \kappa_{0}$ для некоторого фиксированного $\kappa_{0}>0$, и не будем следить за зависимостью констант от $\kappa_{0}$. Таким образом, мы докажем следующий частный случай теоремы 2.5.

Теорема 2.9. Пусть выполнены предположения теоремы 2.5. Для некоторого $\kappa_{0}>0$ определим следуюшую область в $\mathbb{C}$ :

$D:=D_{\kappa_{0}}=\left\{z=E+i \eta \in \mathbb{C}:|z| \leqslant Q, \eta>0,|| E|-2| \geqslant \kappa_{0}, \sqrt{M \eta} \geqslant(\log N)^{C_{1}}\right\}$,

где $C_{1}$ и $Q$ являются достаточно большими. Тогда существуют константы $C_{2}, C$ и с>0, зависящие только от $\vartheta, \kappa_{0} u \delta_{-}$в (2.30), такие, что диагональные матричные элементы $G_{i i}(z)$ функиии Грина удовлетворяют неравенству

$$
\mathrm{P}\left(\bigcup_{z \in D}\left\{\max _{i}\left|G_{i i}(z)-m_{\mathrm{sc}}(z)\right| \geqslant \frac{(\log N)^{C_{2}}}{\sqrt{M \eta}}\right\}\right) \leqslant C N^{-c \log \log N},
$$

а для внедиагональных элементов мы имеем

$$
\mathrm{P}\left(\bigcup_{z \in D}\left\{\max _{i \neq j}\left|G_{i j}(z)\right| \geqslant \frac{(\log N)^{C_{2}}}{\sqrt{M \eta}}\right\}\right) \leqslant C N^{-c \log \log N}
$$

при достаточно большом $N$. 
Мы начнем с системы самосогласованных уравнений на диагональные матричные элементы резольвенты. Следующая лемма является простой комбинацией резольвентных соотношений из п. 2.1.

Лемма 2.10. Диагональные матричные элементы резолъвенты $G_{i i}=$ $(H-z)^{-1}(i, i)$ удовлетворяют следующей системе самосогласованных уравнений:

$$
G_{i i}=\frac{1}{-z-\sum_{j} \sigma_{i j}^{2} G_{j j}+\Upsilon_{i}}
$$

¿əe

$$
\begin{gathered}
\Upsilon_{j}:=A_{i}+h_{i i}-Z_{i}, \\
A_{i}:=\sigma_{i i}^{2} G_{i i}+\sum_{j \neq i} \sigma_{i j}^{2} \frac{G_{i j} G_{j i}}{G_{i i}}, \\
Z_{i}:=Z_{i i}^{(i)}-\mathrm{E}_{i} Z_{i i}^{(i)}, \quad Z_{i i}^{(i)}:=\mathbf{a}^{i} \cdot G^{(i)} \mathbf{a}^{i}=\sum_{k, l \neq i} \overline{\mathbf{a}_{k}^{i}} G_{k l}^{(i)} \mathbf{a}_{l}^{i} .
\end{gathered}
$$

ДокАЗАТЕЛЬСтво. Введем обозначения

$$
Z_{i j}^{(i j)}:=\mathbf{a}^{i} \cdot G^{(i j)} \mathbf{a}^{j}, \quad Z_{i i}^{(i)}:=\mathbf{a}^{i} \cdot G^{(i)} \mathbf{a}^{i} .
$$

Тогда из (2.6) получаем

$$
K_{i j}^{(i j)}=h_{i j}-z \delta_{i j}-Z_{i j}^{(i j)}, \quad K_{i i}^{(i)}=h_{i i}-z-Z_{i i}^{(i)} .
$$

Мы можем записать элемент $G_{i i}$ следующим образом (см. (2.9)):

$$
G_{i i}=\left(K_{i i}^{(i)}\right)^{-1}=\frac{1}{\mathrm{E}_{\mathbf{a}^{i}} K_{i i}^{(i)}+K_{i i}^{(i)}-\mathrm{E}_{\mathbf{a}^{i}} K_{i i}^{(i)}},
$$

где $\mathrm{E}_{\mathbf{a}^{i}}=\mathrm{E}_{i}$ обозначает математическое ожидание по отношению к элементам в $i$-м столбце матрицы $H$.

Используя тот факт, что $G^{(i)}=\left(H^{(i)}-z\right)^{-1}$ не зависит от $\mathbf{a}^{i}$, а $\mathbf{E}_{\mathbf{a}^{i}} \overline{\mathbf{a}_{k}^{i}} \mathbf{a}_{l}^{i}=$ $\delta_{k l} \sigma_{i k}^{2}$, мы находим, что

$$
\mathrm{E}_{\mathbf{a}^{i}} K_{i i}^{(i)}=-z-\sum_{j \neq i} \sigma_{i j}^{2} G_{j j}^{(i)}
$$

И

$$
K_{i i}^{(i)}-\mathrm{E}_{\mathbf{a}^{i}} K_{i i}^{(i)}=h_{i i}-Z_{i}
$$

Используя соотношение

$$
G_{k l}=G_{k l}^{(i)}+\frac{G_{k i} G_{i l}}{G_{i i}}
$$

из $(2.10)$ и обозначения из (2.49), мы получаем выражение

$$
\mathrm{E}_{\mathbf{a}^{i}} K_{i i}^{(i)}=-z-\sum_{j \neq i} \sigma_{i j}^{2} G_{j j}^{(i)}=-z-\sum_{j \neq i} \sigma_{i j}^{2} G_{j j}+\sum_{j \neq i} \sigma_{i j}^{2} \frac{G_{j i} G_{i j}}{G_{i i}}=-z-\sum_{j} \sigma_{i j}^{2} G_{j j}+A_{i} .
$$

Подставляя это в (2.52), мы получаем требуемые уравнения (2.47) из (2.51). 
Введем обозначения

$$
v_{i}:=G_{i i}-m_{\mathrm{sc}}, \quad m:=\frac{1}{N} \sum_{i} G_{i i}, \quad[v]:=\frac{1}{N} \sum_{i} v_{i}=\frac{1}{N} \sum_{i}\left(G_{i i}-m_{\mathrm{sc}}\right) .
$$

Мы будем оценивать следующие ключевые величины:

$$
\Lambda_{\mathrm{d}}:=\max _{k}\left|v_{k}\right|=\max _{k}\left|G_{k k}-m_{\mathrm{sc}}\right|, \quad \Lambda_{\mathrm{o}}:=\max _{k \neq \ell}\left|G_{k \ell}\right|,
$$

где нижний индекс у $\Lambda_{\mathrm{d}}$ означает “диагональные" матричные элементы, а нижний индекс у $\Lambda_{0}$ - "внедиагональные" ("offdiagonal") элементы. Все величины, введенные до сих пор, зависели от спектрального параметра $z=E+i \eta$, но мы, как правило, не будем отмечать это в обозначениях. Вещественная часть энергии $E$ будет всегда оставаться фиксированной. Для мнимой части мы воспользуемся рассуждением с непрерывностью в конце доказательства, а затем опишем зависимость величин $\Lambda_{d}$ и $\Lambda_{o}$ от $z$.

Обе величины $\Lambda_{\mathrm{d}}$ и $\Lambda_{\mathrm{o}}$ будут, как правило, малы при $z \in D$; позднее мы докажем, что их порядок будет меньше $(M \eta)^{-1 / 2}$, по модулю логарифмических поправок. Таким образом, мы можем определить исключительное событие

$$
\Omega_{\Lambda}=\Omega_{\Lambda}(z):=\left\{\Lambda_{\mathrm{d}}(z)+\Lambda_{\mathrm{o}}(z) \geqslant(\log N)^{-C}\right\}
$$

для некоторого $C$ (в этом изложении мы не будем заботиться о согласовании всех показателей). Мы всегда будем работать в $\Omega_{\Lambda}^{c}$, и, в частности, будет иметь место соотношение

$$
\Lambda_{\mathrm{d}}(z)+\Lambda_{\mathrm{o}}(z) \ll 1
$$

Из явной формулы для $m_{\mathrm{sc}}$ легко вытекает, что

$$
c \leqslant\left|m_{\mathrm{sc}}(z)\right| \leqslant C, \quad z \in D,
$$

с некоторыми положительными константами. Следовательно, из соотношения $G_{i i}=m_{\mathrm{sc}}(z)+O\left(\Lambda_{\mathrm{d}}\right)$ мы получаем

$$
c \leqslant\left|G_{i i}(z)\right| \leqslant C, \quad z \in D .
$$

Соотношение (2.11), а именно,

$$
G_{k l}^{(i)}=G_{k l}-\frac{G_{k i} G_{i l}}{G_{i i}}, \quad i \neq l, k,
$$

вместе с (2.55) показывает, что для любых $i$ и $z \in D$ имеют место неравенства

$$
\begin{gathered}
\max _{k \neq l}\left|G_{k l}^{(i)}\right| \leqslant \Lambda_{\text {о }}+C \Lambda_{\mathrm{o}}^{2} \leqslant C \Lambda_{\text {о }} \quad \text { в } \Omega_{\Lambda}^{c}, \\
c \leqslant\left|G_{k k}^{(i)}\right| \leqslant C \quad \text { для всех } k \neq i \text { и в } \Omega_{\Lambda}^{c}, \\
\left|G_{k k}^{(i)}-m_{\mathrm{sc}}\right| \leqslant \Lambda_{\mathrm{d}}+C \Lambda_{\text {о }}^{2} \text { для всех } k \neq i \text { и в } \Omega_{\Lambda}^{c}
\end{gathered}
$$

и $($ см. $(2.49))$

$$
\left|A_{i}\right| \leqslant \frac{C}{M}+C \Lambda_{\mathrm{O}}^{2} \quad \text { в } \Omega_{\Lambda}^{c}
$$


Аналогично, производя еще один шаг в разложении, мы получаем, по-прежнему для $z \in D$ :

$$
\max _{i j} \max _{k \neq l}\left|G_{k l}^{(i j)}\right| \leqslant C \Lambda_{\mathrm{o}}, \quad \max _{i j} \max _{k}\left|G_{k k}^{(i j)}\right| \leqslant C \quad \text { в } \Omega_{\Lambda}^{c}
$$

и

$$
\left|G_{k k}^{(i j)}-m_{\mathrm{sc}}\right| \leqslant \Lambda_{\mathrm{d}}+C \Lambda_{\mathrm{o}}^{2} \quad \text { для всех } k \neq i, j \text { и в } \Omega_{\Lambda}^{c} .
$$

Эти оценки используются в следующей лемме, которая показывает, что величины $Z_{i}$ и $Z_{i j}^{(i j)}$ малы в предположении, что величина $\Lambda_{\mathrm{d}}+\Lambda_{\mathrm{o}}$ мала, a $h_{i j}$ не слишком велики. Это имеет место равномерно в $D$.

Лемма 2.11. Определим исключительные события

$$
\begin{aligned}
\Omega_{1} & :=\left\{\max _{1 \leqslant i, j \leqslant N}\left|h_{i j}\right| \geqslant(\log N)^{C}\left|\sigma_{i j}\right|\right\}, \\
\Omega_{\mathrm{d}}(z) & :=\left\{\max _{i}\left|Z_{i}(z)\right| \geqslant \frac{(\log N)^{C}}{\sqrt{M \eta}}\right\}, \\
\Omega_{\mathrm{o}}(z) & :=\left\{\max _{i \neq j}\left|Z_{i j}^{(i j)}(z)\right| \geqslant \frac{(\log N)^{C}}{\sqrt{M \eta}}\right\}
\end{aligned}
$$

и рассмотрим множество всех исключительных событий

$$
\Omega:=\Omega_{1} \cup \bigcup_{z \in D}\left[\left(\Omega_{\mathrm{d}}(z) \cup \Omega_{\mathrm{o}}(z)\right) \cap \Omega_{\Lambda}^{c}(z)\right]
$$

Тогда

$$
\mathrm{P}(\Omega) \leqslant C N^{-c \log \log N} .
$$

ДокАЗАТЕЛЬСТво. По аналогии с (2.22), при условии (2.32) мы имеем

$$
\mathrm{P}\left(\Omega_{1}\right) \leqslant C N^{-c \log \log N}
$$

так что мы можем работать в дополнительном множестве $\Omega_{1}^{c}$ и предположить, что

$$
\max _{i j}\left|h_{i j}\right| \leqslant \frac{(\log N)^{C}}{\sqrt{M}} .
$$

Теперь мы докажем, что для любого фиксированного $z \in D$ имеют место неравенства

$$
\begin{gathered}
\mathrm{P}\left(\Omega_{\Lambda}^{c}(z) \cap\left\{\max _{i}\left|Z_{i}(z)\right| \geqslant \frac{(\log N)^{C}}{\sqrt{M \eta}}\right\}\right) \leqslant C N^{-c \log \log N}, \\
\mathrm{P}\left(\Omega_{\Lambda}^{c}(z) \cap\left\{\max _{i \neq j}\left|Z_{i j}^{(i j)}(z)\right| \geqslant \frac{(\log N)^{C}}{\sqrt{M \eta}}\right\}\right) \leqslant C N^{-c \log \log N .}
\end{gathered}
$$

Напомним, что $Z^{(i j)}$ является квадратичной формой от компонент случайных векторов $\mathbf{a}^{i}$ и $\mathbf{a}^{j}$. Для таких функций имеется следующий общий результат о больших уклонениях. Его доказательство опирается на неравенство Буркхольдера для мартингалов, оно будет приведено в приложении А. 
Лемма 2.12 [9; леммы В.1, В.2]. Рассмотрим $N$ независимых случайных комплексных величин $a_{i}(1 \leqslant i \leqslant N)$ с нулевым математическим ожиданием, дисперсией $\sigma^{2}$ и имеющих равномерно субэкспоненциальное убывание, т.е.

$$
\mathrm{P}\left(\left|a_{i}\right| \geqslant x^{\alpha}\right) \leqslant C e^{-x}
$$

для некоторого положительного $\alpha$ и для всех $x$. Пусть $A_{i}, B_{i j} \in \mathbb{C}(1 \leqslant$ $i, j \leqslant N)$. Тогда

$$
\begin{aligned}
& \mathrm{P}\left\{\left|\sum_{i=1}^{N} a_{i} A_{i}\right| \geqslant(\log N)^{3 / 2+\alpha} \sigma\left(\sum_{i}\left|A_{i}\right|^{2}\right)^{1 / 2}\right\} \leqslant C N^{-\log \log N}, \\
& \mathrm{P}\left\{\left|\sum_{i=1}^{N} \bar{a}_{i} B_{i i} a_{i}-\sum_{i=1}^{N} \sigma^{2} B_{i i}\right| \geqslant(\log N)^{3 / 2+2 \alpha} \sigma^{2}\left(\sum_{i=1}^{N}\left|B_{i i}\right|^{2}\right)^{1 / 2}\right\} \leqslant C N^{-\log \log N}, \\
& \mathrm{P}\left\{\left|\sum_{i \neq j} \bar{a}_{i} B_{i j} a_{j}\right| \geqslant(\log N)^{3+2 \alpha} \sigma^{2}\left(\sum_{i \neq j}\left|B_{i j}\right|^{2}\right)^{1 / 2}\right\} \leqslant C N^{-\log \log N}
\end{aligned}
$$

Убедимся, что выполнено неравенство (2.65). Применяя оценку (2.69), получим, что неравенство

$$
\left|Z_{i}\right| \leqslant(\log N)^{C}\left(\sum_{k, l \neq i}\left|\sigma_{i k} G_{k l}^{(i)} \sigma_{l i}\right|^{2}\right)^{1 / 2}
$$

имеет место с вероятностью выше $1-C N^{-c \log \log N}$ для достаточно большого $N$.

Обозначим через $u_{\alpha}^{(i)}$ и $\lambda_{\alpha}^{(i)}(\alpha=1, \ldots, N-1)$ собственные векторы и собственные значения матрицы $H^{(i)}$. Пусть $u_{\alpha}^{(i)}(k)$ обозначает $k$-ю координату вектора $u_{\alpha}^{(i)}$. Тогда, используя неравенства $\sigma_{i l}^{2} \leqslant 1 / M,(2.57)$ и $(2.56)$, мы получаем

$$
\begin{gathered}
\sum_{k, l \neq i}\left|\sigma_{i k} G_{k l}^{(i)} \sigma_{l i}\right|^{2} \leqslant \frac{1}{M} \sum_{k \neq i} \sigma_{i k}^{2} \sum_{l \neq i}\left|G_{k l}^{(i)}\right|^{2} \leqslant \frac{1}{M} \sum_{k \neq i} \sigma_{i k}^{2}\left(\left|G^{(i)}\right|^{2}\right)_{k k} \\
=\frac{1}{M} \sum_{k \neq i} \sigma_{i k}^{2} \sum_{\alpha} \frac{\left|u_{\alpha}^{(i)}(k)\right|^{2}}{\left|\lambda_{\alpha}^{(i)}-z\right|^{2}} \leqslant \frac{1}{M} \sum_{k \neq i} \sigma_{i k}^{2} \frac{\operatorname{Im} G_{k k}^{(i)}(z)}{\eta} \leqslant \frac{C}{M \eta} \quad \text { в } \Omega_{\Lambda}^{c} .
\end{gathered}
$$

Здесь мы ввели обозначение $|A|^{2}:=A^{*} A$ для любой матрицы $A$. Вместе с $(2.71)$ это дает неравенство (2.65) для фиксированного $z$. Оценка (2.66) на внедиагональные элементы (при $i \neq j$ ) доказывается аналогично, с использованием (2.70) вместо (2.69).

Таким образом, мы доказали, что для любого фиксированного $z \in D$ множества $\Omega_{\mathrm{d}}(z)$ и $\Omega_{\mathrm{o}}(z)$ имеют очень малую вероятность. При доказательстве неравенства (2.62) нам, вообе говоря, придется брать объединение несчетного количества событий. Однако легко видеть, что величины $Z_{i}(z)$ и $Z_{i j}^{(i j)}(z)$, определяющие эти события, являются липшицево непрерывными функциями от $z$ с константой Липшица $\eta^{-1} \leqslant N$. Следовательно, оценки этих величин 
на достаточно плотной, но конечной сети точек дадут оценки и для всех $z \in D$. Необходимое число точек является лишь степенью $N$, в то время как оценки на вероятности меньше любой обратной степени $N$. Это завершает доказательство леммы 2.11.

Ключевым шагом в доказательстве теоремы 2.9 является следующая лемма. Она утверждает, что если $\Lambda$ допускает оценку сверху порядка $(\log N)^{-C}$, то эта оценка может быть сильно улучшена и величина $\Lambda$ оказывается намного меньше - порядка $(M \eta)^{-1 / 2}$.

Лемма 2.13. Напомним, что величины $\Lambda_{\mathrm{d}}, \Lambda_{\mathrm{o}}$ u $\Omega$ определяются соотношениями (2.53) и (2.61). Мы также будем использовать множество $D$ из (2.44). Тогда для любого $z \in D$ и в $\Omega^{c}$ мы имеем следующую импликацию. Если

$$
\Lambda_{\mathrm{o}}(z)+\Lambda_{\mathrm{d}}(z) \leqslant(\log N)^{-C}
$$

mo

$$
\Lambda_{\mathrm{o}}(z)+\Lambda_{\mathrm{d}}(z) \leqslant \frac{(\log N)^{C}}{\sqrt{M \eta}} .
$$

ДоКАЗАТЕЛЬСТво леммы 2.13. Выбирая $C_{1}$ в (2.44) достаточно большим, мы можем при помощи леммы 2.11 обеспечить выполнение оценок

$$
Z_{i j}^{(i j)} \ll 1, \quad Z_{i} \ll 1 \quad \text { в } \Omega^{c} .
$$

Вначале оценим внедиагональный элемент $G_{i j}$. Если выполнено условие $(2.73)$, то из $(2.8),(2.55)$ и $(2.56)$ мы получаем

$$
\left|G_{i j}\right|=\left|G_{i i}\right|\left|G_{j j}^{(i)}\right|\left|K_{i j}^{(i j)}\right| \leqslant C\left(\left|h_{i j}\right|+\left|Z_{i j}^{(i j)}\right|\right), \quad i \neq j .
$$

Из леммы 2.11 при условии (2.73) мы имеем

$$
\left|G_{i j}\right| \leqslant \frac{(\log N)^{C}}{\sqrt{M}}+\frac{(\log N)^{C}}{\sqrt{M \eta}} \leqslant \frac{(\log N)^{C}}{\sqrt{M \eta}} \quad \text { в } \Omega^{c} .
$$

Это доказывает оценку (2.74) для слагаемого $\Lambda_{\mathrm{o}}$.

Теперь дадим оценку для диагональных элементов. Для величины $\Upsilon_{i}=$ $A_{i}+h_{i i}-Z_{i}$ из $(2.48)$, используя $(2.58),(2.75)$ и лемму 2.11 , мы получаем

$$
\Upsilon:=\max _{i}\left|\Upsilon_{i}\right| \leqslant C \frac{(\log N)^{C}}{\sqrt{M}}+\frac{(\log N)^{C}}{\sqrt{M \eta}} \leqslant \frac{(\log N)^{C}}{\sqrt{M \eta}} \ll 1 \quad \text { в } \Omega^{c}
$$

(на последнем шаге мы использовали тот факт, что $z \in D$ и что $C_{1}$ велико). Из (2.47) вытекает соотношение

$$
v_{i}=G_{i i}-m_{\mathrm{sc}}=\frac{1}{-z-m_{\mathrm{sc}}-\left(\sum_{j} \sigma_{i j}^{2} v_{j}-\Upsilon_{i}\right)}-m_{\mathrm{sc}} .
$$

Используя соотношение $\left(m_{\mathrm{sc}}+z\right)=-m_{\mathrm{sc}}^{-1}$ и неравенства $\left|m_{\mathrm{sc}}+z\right| \geqslant 1$ и $\Lambda_{\mathrm{d}}+$ $\Upsilon \ll 1$, мы можем разложить (2.77) как

$$
\begin{aligned}
v_{i} & =m_{\mathrm{sc}}^{2}\left(\sum_{j} \sigma_{i j}^{2} v_{j}-\Upsilon_{i}\right)+O\left(\sum_{j} \sigma_{i j}^{2} v_{j}-\Upsilon_{i}\right)^{2} \\
& =m_{\mathrm{sc}}^{2}\left(\sum_{j} \sigma_{i j}^{2} v_{j}-\Upsilon_{i}\right)+O\left(\left(\Lambda_{\mathrm{d}}+\Upsilon\right)^{2}\right) .
\end{aligned}
$$


Суммируя выражения из предыдущей формулы по всем $i$ и вспоминая определение $[v]:=\frac{1}{N} \sum_{i} v_{i}=m-m_{\mathrm{sc}}$, мы получаем

$$
[v]=m_{\mathrm{sc}}^{2}(z)[v]-\frac{m_{\mathrm{sc}}^{2}(z)}{N} \sum_{i} \Upsilon_{i}+O\left(\left(\Lambda_{\mathrm{d}}+\Upsilon\right)^{2}\right)
$$

Вводя для простоты обозначения $\zeta:=m_{\mathrm{sc}}^{2}(z),[\Upsilon]:=\frac{1}{N} \sum_{i} \Upsilon_{i}$, мы получаем (используя неравенства $\Lambda_{\mathrm{d}} \leqslant 1$ и $|\zeta| \leqslant 1$ ), что

$$
(1-\zeta)[v]=-\zeta[\Upsilon]+O\left(\left(\Lambda_{\mathrm{d}}+\Upsilon\right)^{2}\right)=O\left(\Lambda_{\mathrm{d}}^{2}+\Upsilon\right)
$$

Напомним, что $\Sigma$ обозначает матрицу ковариаций: $\Sigma_{i j}=\sigma_{i j}^{2}$. Мы знаем, что 1 является простым собственным значением матрицы $\Sigma$, а соответствующим собственным вектором является постоянный вектор $\mathbf{e}=N^{-1 / 2}(1, \ldots, 1)$. Рассмотрим оператор $Q:=I-|\mathbf{e}\rangle\langle\mathbf{e}|$ проектирования на ортогональное дополнение вектора е. Заметим, что $\Sigma$ и $Q$ коммутируют. Пусть $\|\cdot\|_{\infty \rightarrow \infty}$ обозначает матричную норму $\ell^{\infty} \rightarrow \ell^{\infty}$. Используя эти обозначения, мы получаем из $(2.79)$ и $(2.78)$, что

$$
v_{i}-[v]=\zeta \sum_{j} \Sigma_{i j}\left(v_{j}-[v]\right)-\zeta\left(\Upsilon_{i}-[\Upsilon]\right)+O\left(\Lambda_{\mathrm{d}}^{2}+\Upsilon\right)
$$

Суммируя по всем $i$, видим, что суммы всех трех явных членов равны нулю, поэтому сумма поправочных членов также равна нулю. Следовательно, оператор $Q$ действует тождественно на векторе поправочных членов, и мы имеем

$$
\begin{aligned}
v_{i}-[v] & =-\sum_{j}\left(\frac{\zeta}{1-\zeta \Sigma}\right)_{i j}\left(\Upsilon_{j}-[\Upsilon]\right)+O\left(\left\|\frac{\zeta Q}{1-\zeta \Sigma}\right\|_{\infty \rightarrow \infty}\left(\Lambda_{\mathrm{d}}^{2}+\Upsilon\right)\right) \\
& =\left\|\frac{\zeta Q}{1-\zeta \Sigma}\right\|_{\infty \rightarrow \infty} O\left(\Lambda_{\mathrm{d}}^{2}+\Upsilon\right) .
\end{aligned}
$$

Сравнивая (2.79) с (2.80), мы получаем

$$
\Lambda_{\mathrm{d}}=\max _{i}\left|v_{i}\right| \leqslant C\left(\left\|\frac{\zeta Q}{1-\zeta \Sigma}\right\|_{\infty \rightarrow \infty}+\frac{1}{|1-\zeta|}\right)\left(\Lambda_{\mathrm{d}}^{2}+\Upsilon\right) .
$$

Из этих вычислений видно, как оценки ухудшаются на границах. Легко проверить, что

$$
1-\zeta=1-m_{\mathrm{sc}}^{2}(z) \sim \sqrt{\kappa+\eta}, \quad z=E+i \eta, \quad \kappa=|| E|-2| .
$$

Однако, как отмечено в начале доказательства, мы предполагаем для простоты, что $\kappa \geqslant \kappa_{0}>0$, и не следим за этим ухудшением оценок.

Для того чтобы оценить норму резольвенты, мы вспомним следующую элементарную лемму. 
Лемма 2.14 [9; лемма 5.3]. Пусть $\delta_{-}>0$ - некоторая константа. Тогда существуют малье вещественные числа $\tau \geqslant 0$ и $c_{1}>0$, зависящие только от $\delta_{-}$, такие, что для любого положительного числа $\delta_{+}$мы имеем

$$
\max _{x \in\left[-1+\delta_{-}, 1-\delta_{+}\right]}\left\{\left|\tau+x m_{\mathrm{sc}}^{2}(z)\right|^{2}\right\} \leqslant\left(1-c_{1} q(z)\right)(1+\tau)^{2},
$$

где

$$
q(z):=\max \left\{\delta_{+},\left|1-\operatorname{Re} m_{\mathrm{sc}}^{2}(z)\right|\right\} .
$$

ЛЕмма 2.15. Предположим, что для матрищъ $\Sigma$ выполнено условие (2.29), m.e. $\operatorname{Spec}(Q \Sigma) \subset\left[-1+\delta_{-}, 1-\delta_{+}\right]$. Тогда

$$
\left\|\frac{Q}{1-m_{\mathrm{sc}}^{2}(z) \Sigma}\right\|_{\infty \rightarrow \infty} \leqslant \frac{C\left(\delta_{-}\right) \log N}{q(z)}
$$

где $C\left(\delta_{-}\right)$- некоторая константа, зависящая от $\delta_{-}$, a q определяется формулой (2.83).

Из этой леммы можно вывести, что ухудшение оценок на границах, вызванное первым членом в правой части формулы (2.81), может быть устранено предположением $\delta_{+}>0$.

ДоКАЗАТЕЛЬСТВо ЛЕммы 2.15. Обозначим $\|\cdot\|$ обычную матричную норму $\ell^{2} \rightarrow \ell^{2}$ и напомним, что $\zeta=m_{\mathrm{sc}}^{2}(z)$. Запишем

$$
\left\|\frac{Q}{1-\zeta \Sigma}\right\|=\frac{1}{1+\tau}\left\|\frac{Q}{1-(\zeta \Sigma+\tau) /(1+\tau)}\right\|,
$$

где $\tau$ задано в (2.82). Из (2.82) мы получаем

$$
\left\|\frac{\zeta \Sigma+\tau}{1+\tau} Q\right\| \leqslant \sup _{x \in\left[-1+\delta_{-}, 1-\delta_{+}\right]}\left|\frac{\zeta x+\tau}{1+\tau}\right| \leqslant\left(1-c_{1} q(z)\right)^{1 / 2} .
$$

Чтобы оценить норму $\ell^{\infty} \rightarrow \ell^{\infty}$ этой матрицы, напомним, что $|\zeta| \leqslant 1$ и $\sum_{j}\left|\Sigma_{i j}\right|=$
$\sum \sigma_{i j}^{2}=1$. Следовательно, $\sum_{j} \sigma_{i j}^{2}=1$. Следовательно,

$$
\begin{aligned}
\left\|\frac{\zeta \Sigma+\tau}{1+\tau} Q\right\|_{\infty \rightarrow \infty} & =\max _{i} \sum_{j}\left|\left(\frac{\zeta \Sigma+\tau}{1+\tau}\right)_{i j}\right| \\
& \leqslant \frac{1}{1+\tau} \max _{i} \sum_{j}\left|\zeta \Sigma_{i j}+\tau \delta_{i j}\right| \leqslant \frac{|\zeta|+\tau}{1+\tau} \leqslant 1 .
\end{aligned}
$$

Для того чтобы установить неравенство (2.84), мы рассмотрим следующее разложение вплоть до произвольного порога $n_{0}$ :

$$
\begin{aligned}
& \left\|\frac{1}{1-(\zeta \Sigma+\tau) /(1+\tau)} Q\right\|_{\infty \rightarrow \infty} \\
& \leqslant \sum_{n<n_{0}}\left\|\frac{\zeta \Sigma+\tau}{1+\tau} Q\right\|_{\infty \rightarrow \infty}^{n}+\sum_{n \geqslant n_{0}}\left\|\left(\frac{\zeta \Sigma+\tau}{1+\tau}\right)^{n} Q\right\|_{\infty \rightarrow \infty} \\
& \leqslant n_{0}+\sqrt{N} \sum_{n \geqslant n_{0}}\left\|\left(\frac{\zeta \Sigma+\tau}{1+\tau}\right)^{n} Q\right\|=n_{0}+\sqrt{N} \sum_{n \geqslant n_{0}}\left(1-c_{1} q(z)\right)^{n / 2} \\
& =n_{0}+C \sqrt{N} \frac{\left(1-c_{1} q(z)\right)^{n_{0} / 2}}{q(z)} \leqslant \frac{C \log N}{q(z)} .
\end{aligned}
$$


Полагая теперь $n_{0}=(C \log N) / q(z)$, мы завершаем доказательство леммы 2.15 .

Теперь вернемся к доказательству леммы 2.13. Напомним, что мы находимся в множестве $\Omega^{c}$ и что $\kappa \geqslant \kappa_{0}$. Следовательно, функция $1-\operatorname{Re} m_{\mathrm{sc}}^{2}(z)$, а значит, и $q(z)$, отделена от нуля. Подставляя (2.84) в (2.81), мы получаем

$$
\Lambda_{\mathrm{d}} \leqslant C\left(\Lambda_{\mathrm{d}}^{2}+\Upsilon\right) \log N
$$

В силу предположения (2.73), мы имеем $C \Lambda_{\mathrm{d}} \log N \leqslant 1 / 2$. Следовательно, квадратичный член может быть поглощен линейным членом в правой части, и мы имеем

$$
\Lambda_{\mathrm{d}} \leqslant C \Upsilon \log N
$$

Используя оценку (2.76) на $\Upsilon$, мы получаем

$$
\Lambda_{\mathrm{d}} \leqslant \frac{(\log N)^{C}}{\sqrt{M \eta}}
$$

Вместе с (2.75) это завершает доказательство неравенства (2.74). Тем самым лемма 2.13 доказана.

ДОКАЗАТЕЛЬСТвО ТЕОРЕМЫ 2.9. Вводя функции

$$
R(z):=(\log N)^{-C}, \quad S(z):=\frac{(\log N)^{C}}{\sqrt{M \eta}},
$$

мы можем переформулировать лемму 2.13 следующим образом: при событии $\Omega^{c}$, если $\Lambda_{\mathrm{d}}(z)+\Lambda_{\mathrm{o}}(z) \leqslant R(z)$ выполнено для некоторого $z \in D$, то $\Lambda_{\mathrm{d}}(z)+$ $\Lambda_{\mathrm{o}}(z) \leqslant S(z)$. В силу предположения (2.44) теоремы 2.9 , мы имеем $S(z)<R(z)$ для любого $z \in D$. Ясно, что $\Lambda_{\mathrm{d}}(z)+\Lambda_{\mathrm{o}}(z) \leqslant 3 / \eta \leqslant 3 / 10$ при $\operatorname{Im} z=10$. Используя эту информацию, мы можем повторить доказательства лемм 2.11 и 2.13, получив априорную оценку $\Lambda_{\mathrm{d}}(z)+\Lambda_{\mathrm{o}}(z) \leqslant R(z)$ при $\eta=10$. Далее мы воспользуемся непрерывностью функций $R(z), S(z), \Lambda_{\mathrm{d}}(z)$ и $\Lambda_{\mathrm{o}}(z)$. Непрерывно двигая $z$ к вещественной оси, мы получаем, что $\Lambda_{\mathrm{d}}(z)+\Lambda_{\mathrm{o}}(z) \leqslant S(z)$ в $\Omega^{c}$, при соблюдении условия (2.44) для $z$. Это завершает доказательство теоремы 2.9 .

2.3.3. Набросок доказательства полукругового закона для преобразования Стилтьеса. В этом пункте мы уточним оценку, даваемую теоремой 2.9 для преобразования Стилтьеса $m(z)=\frac{1}{N} \sum_{i} G_{i i}$. Ключевым улучшением является то, что величина $\left|m-m_{\mathrm{sc}}\right|$ будет оценена с точностью $(M \eta)^{-1}$, в то время как величина $\left|G_{i i}-m_{\mathrm{sc}}\right|$ оценивалась лишь с точностью $(M \eta)^{-1 / 2}$ (по модулю логарифмических членов, отражающих ухудшение оценки вблизи границ). В следующей теореме мы докажем частный случай неравенства (2.34) из теоремы 2.5 .

ТЕОРема 2.16. Пусть выполнены условия теоремы 2.5. Зафиксируем $\kappa_{0}>0$ и рассмотрим область $D=D_{\kappa_{0}}$ из (2.44). Тогда для любъх $\varepsilon>0$ u $K>0$ существует константа $C=C\left(\varepsilon, K, \kappa_{0}\right)$ такая, что

$$
\mathrm{P}\left(\bigcup_{z \in D}\left\{\left|m(z)-m_{\mathrm{sc}}(z)\right| \geqslant \frac{N^{\varepsilon}}{M \eta}\right\}\right) \leqslant \frac{C\left(\varepsilon, K, \kappa_{0}\right)}{N^{K}} .
$$


ДоКАЗАТЕЛЬСТво тЕОРЕмы 2.16. Мы в основном следуем доказательству теоремы 2.9. Выберем $z \in D$ и будем работать на дополнении к событиям малой вероятности с оценками (2.45) и (2.46). В частности, имеет место оценка $(2.74)$. Как и в (2.79), мы имеем

$$
[v]=m-m_{\mathrm{sc}}=-\frac{\zeta}{1-\zeta} \frac{1}{N} \sum_{i} \Upsilon_{i}+O\left(\left(\Lambda_{\mathrm{d}}+\Upsilon\right)^{2}\right)
$$

с очень большой вероятностью. Однако теперь для первого члена мы дадим более точную оценку, чем самая тривиальная оценка, использованная в (2.79). Это приведет к некоторым сокращениям из флуктуирующей суммы. Тогда из (2.76) и (2.85) мы получаем, что соотношение

$$
m-m_{\mathrm{sc}}=O\left(\frac{1}{N} \sum_{j} \Upsilon_{j}\right)+O\left(\frac{N^{\varepsilon}}{M \eta}\right)
$$

имеет место с очень высокой вероятностью для любого малого $\varepsilon>0$. Напомним, что $\Upsilon_{i}=A_{i}+h_{i i}-Z_{i}$. Из $(2.49),(2.55)$ и неравенства $\sigma_{i j}^{2} \leqslant M^{-1}$ мы получаем

$$
A_{j} \leqslant \frac{C}{M}+C \Lambda_{\mathrm{o}}^{2} \leqslant \frac{C N^{\varepsilon}}{M \eta},
$$

где мы использовали неравенство (2.74) для оценки величины $\Lambda_{\mathrm{o}}$.

Отсюда вытекает, что соотношение

$$
m-m_{\mathrm{sc}}=O\left(\frac{1}{N} \sum_{i} Z_{i}-\frac{1}{N} \sum_{i} h_{i i}\right)+O\left(\frac{N^{\varepsilon}}{M \eta}\right)
$$

имеет место с очень высокой вероятностью. Так как величины $h_{i i}$ независимы, то применяя первую оценку из леммы 2.12 о больших уклонениях, мы получаем

$$
\mathrm{P}\left(\left|\frac{1}{N} \sum_{i} h_{i i}\right| \geqslant(\log N)^{C_{\alpha}} \frac{1}{\sqrt{M N}}\right) \leqslant C N^{-c \log \log N} .
$$

На дополнительном событии мы можем включить оценку $(\log N)^{C_{\alpha}}(M N)^{-1 / 2}$ в последний поправочный член в (2.87). Остается лишь оценить величину

$$
[Z]:=\frac{1}{N} \sum_{i} Z_{i}
$$

Верхняя оценка для ее моментов дана в следующей лемме; ниже мы обсудим ее доказательство.

Лемма 2.17 [8; лемма 5.2], [7; лемма 4.1]. Используя определение величин $Z_{i}$ из (2.50), для любого фиксированного z в области $D$ и любого натурального числа $s$ мы имеем

$$
\mathrm{E}\left|\frac{1}{N} \sum_{i=1}^{N} Z_{i}\right|^{2 s} \leqslant C_{s}\left(\frac{(\log N)^{C}}{M \eta}\right)^{2 s} .
$$


Из этой леммы следует, что для любых $\varepsilon>0$ и $K>0$ справедливо неравенство

$$
\mathrm{P}\left(\frac{1}{N}\left|\sum_{i=1}^{N} Z_{i}\right| \geqslant \frac{N^{\varepsilon}}{M \eta}\right) \leqslant N^{-K}
$$

для достаточно большого $N$. Отсюда и из (2.88) и (2.87) вытекает неравенство (2.86), что завершает доказательство теоремы 2.16.

НАБРОСОК ДОКАЗАТЕЛЬСТВА ЛЕмМЫ 2.17. Мы располагаем двумя доказательствами этой леммы, и оба достаточно сложны. Здесь мы приводим идеи первого доказательства из [8]. Рассуждение заключается в длинном и тщательно организованном вычислении высших моментов, аналогичном оценке на второй момент в (2.23), но теперь нам потребуется выделить дополнительный множитель из суммы. Заметим, что, обобщив вычисление второго момента (2.23) на высшие моменты (и вспомнив, что для универсальных матриц Вигнера $N$ заменяется на $M$ ), мы можем доказать следующее неравенство:

$$
\left|Z_{i}\right| \lesssim \frac{1}{\sqrt{M \eta}}
$$

(доказательство будет обсуждаться ниже в лемме 2.18). Если бы величины $Z_{i}$ были независимыми, то центральная предельная теорема давала бы нам оценку

$$
\left|\frac{1}{N} \sum_{i} Z_{i}\right| \lesssim \frac{1}{\sqrt{N}} \frac{1}{\sqrt{M \eta}}
$$

которой было бы более чем достаточно. В действительности, величины $Z_{i}$ являются не вполне независимыми, но почти. Зависимости между различными $Z_{i}$ могут быть извлечены из резольвентных формул в п. 2.1. Мы дадим набросок вычисления второго момента, т. е. случай $s=1$. Более подробное изложение и вычисление высших моментов дано в разделах 8, 9 статьи [8].

Так как $\mathrm{E} Z_{i}=0$, то дисперсия величины $[Z]$ задается формулой

$$
\frac{1}{N^{2}} \mathrm{E}\left|\sum_{i=1}^{N} Z_{i}\right|^{2}=\frac{1}{N^{2}} \mathrm{E} \sum_{\alpha \neq \beta} \bar{Z}_{\alpha} Z_{\beta}+\frac{1}{N^{2}} \mathrm{E} \sum_{\alpha}\left|Z_{\alpha}\right|^{2} .
$$

Мы начнем с оценки первого члена в правой части (2.91) при $\alpha=1$ и $\beta=2$. Основная идея заключается в том, чтобы представить $G_{k l}^{(1)}$ в виде

$$
G_{k l}^{(1)}=P_{k l}^{(12)}+P_{k l}^{(1)}
$$

где $P_{k l}^{(12)}$ не зависит от $\mathbf{a}^{1}$ и $\mathbf{a}^{2}$, a $P_{k l}^{(1)}$ не зависит от $\mathbf{a}^{1}$ (напомним соглашение об обозначениях: верхний индекс означает независимость от соответствующего столбца матрицы $H$ ). Чтобы построить такое разложение для $k, l \notin\{1,2\}$, мы воспользуемся соотношениями $(2.10)$ или $(2.11)$ и запишем $G_{k l}^{(1)}$ в виде

$$
G_{k l}^{(1)}=G_{k l}^{(12)}+\frac{G_{k 2}^{(1)} G_{2 l}^{(1)}}{G_{22}^{(1)}}, \quad k, l \notin\{1,2\} .
$$


Здесь первое слагаемое в правой части не зависит от $\mathbf{a}^{2}$. Применяя теорему 2.9 к минорам, мы получим, что $G_{k l}^{(1)} \lesssim(M \eta)^{-1 / 2}$ при $k \neq l \neq 1$ и $G_{k k}^{(2)} \geqslant c>0$. Следовательно, мы с очень высокой вероятностью имеем оценку

$$
\left|\frac{G_{k 2}^{(1)} G_{2 l}^{(1)}}{G_{22}^{(1)}}\right| \lesssim \frac{1}{M \eta}
$$

Заметим, что эта оценка представляет собой квадрат оценки $G_{k l}^{(1)} \lesssim(M \eta)^{-1 / 2}$ из теоремы 2.9 .

Теперь определим величины $P^{(1)}$ и $P^{(12)}$ (при $k, l \neq 1$ ) следующим образом:

1) при $k, l \neq 2$

$$
P_{k l}^{(12)}:=G_{k l}^{(12)}, \quad P_{k l}^{(1)}:=\frac{G_{k 2}^{(1)} G_{2 l}^{(1)}}{G_{22}^{(1)}}=G_{k l}^{(1)}-G_{k l}^{(12)}
$$

2) при $k=2$ или $l=2$

$$
P_{k l}^{(12)}:=0, \quad P_{k l}^{(1)}:=G_{k l}^{(1)}
$$

Тогда имеет место равенство $(2.92)$ и $P_{k l}^{(12)}$ не зависит от $\mathbf{a}^{2}$.

Размер квадратичных форм $\mathbf{a}^{1} \cdot P^{(1)} \mathbf{a}^{1}$ и $\mathbf{a}^{1} \cdot P^{(12)} \mathbf{a}^{1}$ может быть оценен при помощи следующей леммы, доказательство которой мы отложим.

ЛЕмма 2.18. При $N^{-1} \leqslant \eta \leqslant 1$ и фиксированном $p \in \mathbb{N}$ мы имеем

$$
\mathrm{E}\left|\mathbf{a}^{1} \cdot P^{(1)} \mathbf{a}^{1}\right|^{p} \lesssim \frac{C_{p}}{(M \eta)^{p}}, \quad \mathrm{E}\left|\mathbf{a}^{1} \cdot P^{(12)} \mathbf{a}^{1}\right|^{p} \lesssim \frac{C_{p}}{(M \eta)^{p / 2}} .
$$

Заметим, что первая квадратичная форма меньше, но вторая не зависит от второго столбца матрицы $H$.

Введем оператор $\mathbb{I E}_{i}:=\mathbb{I}-\mathrm{E}_{\mathbf{a}^{i}}$, где $\mathbb{I}$ - тождественный оператор. Используя это обозначение, мы получаем следующее разложение для $Z_{1}$ :

$$
Z_{1}=\mathbb{I} \mathrm{E}_{1} \mathbf{a}^{1} \cdot P^{(12)} \mathbf{a}^{1}+\mathbb{I} \mathrm{E}_{1} \mathbf{a}^{1} \cdot P^{(1)} \mathbf{a}^{1} .
$$

Поменяв местами индексы 1 и 2 , мы можем определить величины $P^{(21)}$ и $P^{(2)}$ и разложить $Z_{2}$ как

$$
Z_{2}=\mathbb{I} \mathrm{E}_{2} \mathbf{a}^{2} \cdot P^{(21)} \mathbf{a}^{2}+\mathbb{I} \mathrm{E}_{2} \mathbf{a}^{2} \cdot P^{(2)} \mathbf{a}^{2}
$$

Здесь $P_{k l}^{(21)}$ не зависит от $\mathbf{a}^{2}$ и $\mathbf{a}^{1}$, a $P_{k l}^{(2)}$ не зависит от $\mathbf{a}^{2}$. Из $(2.99)$ и (2.98) мы получаем следующее соотношение для смешанного члена, соответствующего значениям $\alpha=1, \beta=2$ в $(2.91)$ :

$$
\mathrm{E} \bar{Z}_{1} Z_{2}=\mathrm{E}\left[\left(\mathbb{I E}_{1}\left\{\overline{\mathbf{a}^{1} \cdot P^{(12)} \mathbf{a}^{1}}+\overline{\mathbf{a}^{1} \cdot P^{(1)} \mathbf{a}^{1}}\right\}\right)\left(\mathbb{I E}_{2}\left\{\mathbf{a}^{2} \cdot P^{(21)} \mathbf{a}^{2}+\mathbf{a}^{2} \cdot P^{(2)} \mathbf{a}^{2}\right\}\right)\right] .
$$

Заметим, что если $X^{(i)}$ является независимой от $\mathbf{a}^{i}$ случайной величиной, то для любой случайной величины $Y$ мы имеем

$$
\mathbb{I E}_{i}\left[Y X^{(i)}\right]=X^{(i)} \mathbb{I E}_{i} Y .
$$


В частности, $\mathbb{I E}_{i} X^{(i)}=0$ (где $\left.Y=1\right)$. Следовательно,

$$
\mathrm{E}\left[\left(\mathbb{I E}_{1} X^{(2)}\right)\left(\mathbb{I E}_{2} X^{(1)}\right)\right]=\mathrm{E}\left[\mathbb{I E}_{1}\left[\left(\mathbb{I E}_{2} X^{(1)}\right) X^{(2)}\right]\right]=0,
$$

так как $\mathrm{EIE}_{i}=0$. Используя эти соображения, легко видеть, что единственным ненулевым членом в правой части соотношения (2.100) будет

$$
\mathrm{E}\left(\mathbb{I E}_{1} \overline{\mathbf{a}^{1} P^{(1)} \mathbf{a}^{1}}\right)\left(\mathbb{I E} \mathrm{E}_{2} \mathbf{a}^{2} P^{(2)} \mathbf{a}^{2}\right) .
$$

Из неравенства Коши-Буняковского и леммы 2.18 мы получаем

$$
\left|\mathrm{E} \bar{Z}_{1} Z_{2}\right| \lesssim \frac{1}{(M \eta)^{2}}
$$

С учетом (2.92), из леммы 2.18 также вытекает неравенство

$$
\mathrm{E}\left|Z_{i}\right|^{p} \lesssim \frac{C_{p}}{(M \eta)^{p / 2}}, \quad 1 \leqslant i \leqslant N
$$

что доказывает (2.90) и дает оценку второго члена в соотношении (2.91) числом $N^{-1}(M \eta)^{-1} \leqslant(M \eta)^{-2}$. Так как индексы 1 и 2 в (2.102) можно заменить на любые индексы $\alpha \neq \beta$, то вместе с (2.91) это дает доказательство леммы 2.17 для $s=1$.

ДокАЗАТЕЛЬСтво лЕммы 2.18. Вначале перепишем выражение $\mathbf{a}^{1} \cdot P^{(1)} \mathbf{a}^{1}$ следующим образом:

$\mathbf{a}^{1} \cdot P^{(1)} \mathbf{a}^{1}=\sum_{k, l \neq 2} \overline{\mathbf{a}_{k}^{1}} \cdot\left(\frac{G_{k 2}^{(1)} G_{2 l}^{(1)}}{G_{2,2}^{(1)}}\right) \mathbf{a}_{l}^{1}+\sum_{k \neq 2} \overline{\mathbf{a}_{k}^{1}} \cdot G_{k 2}^{(1)} \mathbf{a}_{2}^{1}+\sum_{l \neq 2} \overline{\mathbf{a}_{2}^{1}} \cdot G_{2 l}^{(1)} \mathbf{a}_{l}^{1}+\overline{\mathbf{a}_{2}^{1}} G_{22}^{(1)} \mathbf{a}_{2}^{1}$.

Используя оценку больших уклонений (2.70) и неравенство (2.94), мы получаем

$$
\mathrm{P}\left(\left|\sum_{k, l \neq 2} \overline{\mathbf{a}_{k}^{1}}\left(\frac{G_{k 2}^{(1)} G_{2 l}^{(1)}}{G_{22}^{(1)}}\right) \mathbf{a}_{l}^{1}\right| \geqslant \frac{(\log N)^{C}}{M \eta}\right) \leqslant N^{-c \log \log N}
$$

Аналогично, используя (2.68) и тот факт, что $\left\|\mathbf{a}^{1}\right\|_{\infty} \lesssim M^{-1 / 2}$, мы получаем, что второй и третий члены в (2.104) ограничены сверху числом $\frac{1}{\sqrt{M}} \frac{1}{\sqrt{M \eta}} \lesssim$ $(M \eta)^{-1}$. Последний член имеет даже меньший порядок $1 / M$ с очень высокой вероятностью. Тем самым мы доказали, что

$$
\mathrm{P}\left(\left|\mathbf{a}^{1} \cdot P^{(1)} \mathbf{a}^{1}\right| \leqslant \frac{(\log N)^{C}}{M \eta}\right) \geqslant 1-N^{-c \log \log N} .
$$

Это неравенство дает первое из требуемых неравенств (2.97), вне пределов исключительного множества с вероятностью меньшей, чем любая степень $1 / N$. Так как все функции Грина ограничены сверху числом $\eta^{-1} \leqslant N$, то вкладом исключительного множества можно пренебречь, и это доказывает первую из оценок (2.97). Вторая оценка доказывается аналогично. 
2.4. Сильный локальный полукруговой закон. В этом пункте мы приводим наши последние результаты из [7], которые позволяют избавиться от зависимости от $\kappa$ в теореме 2.5 , теореме 2.6 и теореме 2.7 для ансамблей с $M=N$, в частности, для обобщенных матриц Вигнера. Мы продолжим использовать обозначения

$$
\Lambda_{\mathrm{d}}:=\max _{k}\left|G_{k k}-m_{\mathrm{sc}}\right|, \quad \Lambda_{\mathrm{o}}:=\max _{i \neq j}\left|G_{i j}\right|, \quad \Lambda:=\left|m-m_{\mathrm{sc}}\right|
$$

и напомним, что все эти величины зависят от спектрального параметра $z$ и от $N$.

ТЕОРЕМА 2.19 (сильный локальный полукруговой закон [7; теорема 2.1]). Пусть $H=\left(h_{i j}\right)$ - эрмитова или симметрическая случайная матрица размера $N \times N$, где $N \geqslant 3 u \mathrm{E} h_{i j}=0$ при $1 \leqslant i, j \leqslant N$. Предположим, что дисперсии $\sigma_{i j}^{2}$ удовлетворяют условиям (1.16) и (2.29) с некоторыми положительными константами $\delta_{ \pm}>0$ и имеет место верхняя оценка

$$
\sigma_{i j}^{2} \leqslant \frac{C_{0}}{N}
$$

Предположим, далее, что распределения матричных элементов имеют равномерное субэкспоненциальное убывание, т.е. существуют константы $C, \vartheta>0$, не зависящие от $N$, такие, что для любых $x \geqslant 1 u 1 \leqslant i, j \leqslant N$ выполнено неравенство

$$
\mathrm{P}\left(\left|h_{i j}\right| \geqslant x \sigma_{i j}\right) \leqslant C \exp \left(-x^{\vartheta}\right) .
$$

Тогда для любой константы $\xi>1$ существуют положительные константы L, C и с, зависящие только от $\xi u \vartheta$, от $\delta_{ \pm}$из (2.108), (2.29) и от $C_{0}$ из (2.107), такие, что преобразование Стилтвеса эмпирического распределения собственных значений матрицы Н удовлетворяет неравенству

$$
\mathrm{P}\left(\bigcup_{z \in \mathbf{S}_{L}}\left\{\Lambda(z) \geqslant \frac{(\log N)^{4 L}}{N \eta}\right\}\right) \leqslant C \exp \left[-c(\log N)^{\xi}\right]
$$

zдe

$$
\mathbf{S}:=\mathbf{S}_{L}=\left\{z=E+i \eta:|E| \leqslant 5, N^{-1}(\log N)^{10 L}<\eta \leqslant 10\right\} .
$$

Индивидуальные матричные элементы функиии Грина удовлетворяют неравенству

$$
\begin{aligned}
& \mathrm{P}\left(\bigcup_{z \in \mathbf{S}_{L}}\left\{\Lambda_{\mathrm{d}}(z)+\Lambda_{\mathrm{o}}(z) \geqslant(\log N)^{4 L} \sqrt{\frac{\operatorname{Im} m_{\mathrm{sc}}(z)}{N \eta}}+\frac{(\log N)^{4 L}}{N \eta}\right\}\right) \\
& \quad \leqslant C \exp \left[-c(\log N)^{\xi}\right] .
\end{aligned}
$$

Более того, внутри следующего множества вне ограничивающего спектра

$$
\mathbf{O}_{L}:=\left\{z=E+i \eta: N \eta \sqrt{\kappa} \geqslant(\log N)^{4 L}, \kappa \geqslant \eta,|E|>2\right\}, \quad \text { где } \kappa:=|| E|-2|,
$$

имеет место более сильная оценка

$$
\mathrm{P}\left(\bigcup_{z \in \mathbf{O}_{L}}\left\{\Lambda(z) \geqslant \frac{(\log N)^{4 L}}{N \kappa}\right\}\right) \leqslant C \exp \left[-c(\log N)^{\xi}\right] .
$$


Условие субэкспоненциального убывания (2.108) можно ослабить, если нам не требуются оценки ошибок, которые убывают быстрее, чем любая степень $N$. Эта модификация производится несложным образом, и мы не будем приводить соответствующих рассуждений в этой работе.

До появления наших результатов из [9] и [8] центральная предельная теорема для полукругового закона в макроскопическом масштабе для ленточных матриц была установлена Гионне [59] и Андерсоном-Цайтуни [60]; а полукруговой закон для гауссовых ленточных матриц был доказан Дисертори, Пинсоном и Спенсером в [51]. Обзор результатов о ленточных матрицах дан в недавней работе Спенсера [103].

Как и ранее, из оценок локального полукругового закона вытекает, что эмпирическая функция подсчета собственных значений близка к функции подсчета полукругового закона и что расположение собственных значений близко к их классическому расположению. Мы имеем следующие более сильные результаты (см. теоремы 2.6 и 2.7).

ТеОрема 2.20 [7; теорема 2.2]. Пусть выполнены условия теоремы 2.19, т.е. условия (1.16), (2.29) с некоторыми положительными константами $\delta_{ \pm}>0,(2.107) u(2.108)$. Тогда для любой константы $\xi>1$ существуют константы $L_{1}, C u c>0$, зависящие только от $\xi, \vartheta, \delta_{ \pm} u C_{0}$, такие, чmo

$$
\mathrm{P}\left\{\exists j:\left|\lambda_{j}-\gamma_{j}\right| \geqslant(\log N)^{L_{1}}[\min \{j, N-j+1\}]^{-1 / 3} N^{-2 / 3}\right\} \leqslant C \exp \left[-c(\log N)^{\xi}\right]
$$

$u$

$$
\mathrm{P}\left\{\sup _{|E| \leqslant 5}\left|\mathfrak{n}(E)-n_{\mathrm{sc}}(E)\right| \geqslant \frac{(\log N)^{L_{1}}}{N}\right\} \leqslant C \exp \left[-c(\log N)^{\xi}\right] .
$$

Для матриц Вигнера оценка (2.115), в которой множитель $N^{-1}$ заменен на $N^{-2 / 5}$, была установлена в [104] (в несколько более слабой формулировке), а более сильная оценка с множителем $N^{-1 / 2}$ была доказана для разности $\mathrm{En}(E)-n_{\mathrm{sc}}(E) . \quad$ В теореме 1.3 из [105] была доказана следующая оценка для стандартных матриц Вигнера (в нашей нормировке):

$$
\left(\mathrm{E}\left[\left|\lambda_{j}-\gamma_{j}\right|^{2}\right]\right)^{1 / 2} \leqslant[\min \{j, N-j+1\}]^{-1 / 3} N^{-1 / 6-\varepsilon_{0}}
$$

с некоторым малым положительным $\varepsilon_{0}$, при условии, что третий момент матричных элементов обращается в нуль. В той же работе была сформулирована гипотеза, что множитель $N^{-1 / 6-\varepsilon_{0}}$ в правой части неравенства (2.116) может быть заменен на $N^{-2 / 3+\varepsilon}$. До появления работы [105] оценка (2.114) была доказана в [8] вдали от границ спектра, с несколько более слабой оценкой на вероятность и с множителем $N^{\delta}$ для произвольного $\delta>0$ вместо множителя $(\log N)^{L_{1}}$ (см. уравнение перед (7.8) в [8]).

Заметим, что все результаты сформулированы для эрмитова и симметрического случаев, но формулировки и доказательства проходят и для кватернионных самодвойственных случайных матриц (см., например, [6; раздел 3.1]).

Рассуждения, приведенные в пп. 2.3.2 и 2.3.3, могут быть улучшены в нескольких местах, что приводит к доказательству оптимальной теоремы 2.19 для случая $M=N$. Здесь мы упомянем лишь наиболее важные из этих улучшений. 
Во-первых, заметим, что заключительная часть обоснования оценки (2.72) может быть проведена более эффективно в случае $M=N$ и $\sigma_{i k}^{2} \leqslant C / N$ с использованием неравенства

$$
\frac{1}{N} \sum_{k} \operatorname{Im} G_{k k}^{(i)} \leqslant \frac{1}{N} \sum_{k} \operatorname{Im} G_{k k}+C \Lambda_{\mathrm{o}}^{2}=\operatorname{Im} m+C \Lambda_{\mathrm{o}}^{2} \leqslant \Lambda+\Lambda_{\mathrm{o}}^{2}+\operatorname{Im} m_{\mathrm{sc}} .
$$

Улучшением здесь является то, что $\operatorname{Im} m_{\mathrm{sc}}(z) \sim \sqrt{\kappa+\eta}$. Это дает вблизи границ оценку, более точную, чем просто $O(1)$. Затем мы вводим наш основной контрольный параметр

$$
\Psi=\Psi(z):=\sqrt{\frac{\Lambda(z)+\operatorname{Im} m_{\mathrm{sc}}(z)}{N \eta}},
$$

который хоть и является случайной величиной, но зависит лишь от $\Lambda$. Аналогично тому, как мы доказывали неравенства (2.75) и (2.76), можно доказать, что неравенство

$$
\Lambda_{\mathrm{O}}+\max _{i} \Upsilon_{i} \leqslant \Psi
$$

справедливо с очень большой вероятностью.

Во-вторых, более детальный алгебраический анализ самосогласованного уравнения (2.78) приводит к следующему тождеству:

$$
\left(1-m_{\mathrm{sc}}^{2}\right)[v]=m_{\mathrm{sc}}^{3}[v]^{2}+m_{\mathrm{sc}}^{2}[Z]+O\left(\frac{\Lambda^{2}}{\log N}\right)+O\left((\log N) \Psi^{2}\right),
$$

где $[Z]:=N^{-1} \sum_{i=1}^{N} Z_{i}$. Преимущество этой формулы состоит в том, что она позволяет не только выразить $[v]$ из ее левой части (после деления на $1-m_{\mathrm{sc}}^{2}$ ), но также, в случае выполнения неравенства

$$
\left|\left(1-m_{\mathrm{sc}}^{2}\right)[v]\right| \ll\left|m_{\mathrm{sc}}^{3}[v]^{2}\right|
$$

(что, как правило, имеет место именно вблизи границ, где $1-m_{\mathrm{sc}}^{2}$ мало), выразить $[v]$ из правой части. Это приводит к дихотомической оценке. А именно, заметим, что если имеет место соотношение

$$
\left(1-m_{\mathrm{sc}}^{2}\right)[v]=m_{\mathrm{sc}}^{3}[v]^{2}+\text { малый член, }
$$

или, другими словами,

$$
\alpha(z) \Lambda=\Lambda^{2}+\beta(z), \quad \alpha(z):=\left|\frac{1-m_{\mathrm{sc}}^{2}}{m_{\mathrm{sc}}^{3}}\right| \sim \sqrt{\kappa+\eta}, \quad \beta-\text { малый член }
$$

то для некоторой достаточно большой константы $U$ и другой константы $C_{1}(U)$ мы имеем

$$
\begin{array}{ll}
\Lambda(z) \leqslant U \beta(z) \quad \text { или } \quad \Lambda(z) \geqslant \frac{\alpha(z)}{U} & \text { при } \alpha \geqslant U^{2} \beta, \\
\Lambda(z) \leqslant C_{1}(U) \beta(z) & \text { при } \alpha \leqslant U^{2} \beta .
\end{array}
$$


Плохой случай $\Lambda \geqslant \alpha / U$ может быть исключен при помощи рассуждения с непрерывностью: можно легко показать, что этот случай не имеет места при $\eta=10$, а затем уменьшать $\eta$, замечая, что мы либо все время находимся в первом случае (при условии $\alpha \geqslant U^{2} \beta$ ), либо (при условии $\alpha \leqslant U^{2} \beta$ ) мы сразу получаем $\Lambda \lesssim \beta$. В действительности доказательство несколько сложнее, так как "малый" член сам по себе зависит от $\Lambda$, но эта зависимость может быть включена в другие члены. Более того, все эти оценки имеют место лишь с очень высокой вероятностью, так что необходимо следить за исключительными событиями.

Наконец, оценка из леммы 2.17 должна включить улучшенный контроль на границах. Изучая доказательство, мы видим, что улучшение $(M \eta)^{-2 s}$ происходит из внедиагональных элементов резольвенты. Лемму 2.17 можно сформулировать в следующем виде:

$$
\mathrm{E}\left|\frac{1}{N} \sum_{i=1}^{N} Z_{i}\right|^{2 s} \leqslant C_{s} \mathrm{E}\left[\Lambda_{\mathrm{O}}^{2 s}+N^{-s}\right]
$$

Как и ранее, это неравенство можно превратить в оценку вероятности, взяв большую степень: $s \sim(\log N)^{\xi}$. Используя неравенство $(2.117)$ для оценки $\Lambda_{\text {о }}$ через $\Psi$ и тот факт, что $\Psi^{2} \leqslant o(\Lambda)+N^{-1}$ (так как $N \eta \gg 1$ ), можно показать, что член $m_{\mathrm{sc}}^{2}[Z]$ в правой части соотношения (2.118) также является "малым". Подробности даны в [7; разделы 3 и 4].

2.5. Делокализация собственных векторов. Пусть $H$ - универсальная матрица Вигнера с субэкспоненциальным убыванием (2.32). Рассмотрим $\ell^{2}$-нормированный собственный вектор $\mathbf{v}$ матрицы $H$. Величина $\ell^{p}$-нормы вектора $\mathbf{v}$ при $p>2$ дает информацию о делокализации $\mathbf{v}$. Мы будем говорить, что имеет место полная делокализация, если $\|\mathbf{v}\|_{p} \lesssim N^{-1 / 2+1 / p}$ (заметим, что $\left.\|\mathbf{v}\|_{p} \geqslant C N^{-1 / 2+1 / p}\|\mathbf{v}\|_{2}\right)$. Следующий результат показывает, что для обобщенных матриц Вигнера (1.17) собственные векторы делокализуются с очень высокой вероятностью. Для универсальных матриц Вигнера ширины $M$ (см. (2.31)) собственные векторы делокализуются в масштабе по крайней мере $M$.

ТеОрема 2.21. Пусть выполнены предположения теоремы 2.5. Тогда для любого $E$ с $\kappa=|E-2| \geqslant \kappa_{0}$ мы имеем

$$
\mathrm{P}\left\{\exists \mathbf{v}: H \mathbf{v}=\lambda \mathbf{v},|\lambda-E| \leqslant \frac{1}{M},\|\mathbf{v}\|_{2}=1,\|\mathbf{v}\|_{\infty} \geqslant \frac{(\log N)^{C}}{\sqrt{M}}\right\} \leqslant C N^{-c \log \log N} .
$$

Заметим, что соотношение $\|\mathbf{v}\|_{\infty} \lesssim M^{-1 / 2}$ показывает, что носитель собственного вектора сосредоточен в области размера по крайней мере $M$, т. е. длина локализации вектора $\mathbf{v}$ не меньше $M$. Отметим также, что согласно гипотезе о делокализации длина локализации на самом деле равна $M^{2}$, т. е. оптимальной оценкой должна быть

$$
\|\mathbf{v}\|_{\infty} \lesssim \frac{1}{M}
$$

с очень высокой вероятностью. Как было указано в п. 1.3, этот вопрос остается открытым. Имеются лишь некоторые частные результаты, например, 
в [49], [50] нами было доказано, что для случайных ленточных матриц (1.18) с шириной ленты $W \sim M$ длина локализации равна по крайней мере $M^{1+1 / 6}$.

ДокАЗАтЕЛьство. Мы опять не будем принимать во внимание зависимость оценки от $\kappa_{0}$ (это можно проследить из доказательства). Оценка (2.45) влечет неравенство

$$
\left|G_{i i}(z)\right| \leqslant C
$$

для любого $z=E+i \eta$, где $M \eta \geqslant(\log N)^{C}$, с очень высокой вероятностью. Выберем $\eta=(\log N)^{C} / M$. Пусть $\mathbf{v}_{\alpha}$ обозначают собственные векторы матрицы $H$, и пусть $\mathbf{v}$ - собственный вектор с собственным значением $\lambda$, где $|\lambda-E| \leqslant 1 / M$. Тогда

$$
|\mathbf{v}(i)|^{2} \leqslant \frac{2 \eta^{2}|\mathbf{v}(i)|^{2}}{M^{-2}+\eta^{2}} \leqslant 2 \sum_{\alpha} \frac{\eta^{2}\left|\mathbf{v}_{\alpha}(i)\right|^{2}}{\left(\lambda_{\alpha}-E\right)^{2}+\eta^{2}}=2 \eta\left|\operatorname{Im} G_{i i}\right| \leqslant C \eta
$$

т. e.

$$
\|\mathbf{v}\|_{\infty} \leqslant \frac{(\log N)^{C}}{\sqrt{M}}
$$

Теорема доказана.

Заметим, что доказательство было очень простым, так как имелись поточечные оценки на диагональные элементы резольвенты. Эту теорему можно также доказать, опираясь лишь на локальный полукруговой закон, что является концептуально более простой предпосылкой. Традиционно, мы использовали именно этот путь в [55]-[57]. Например, в [57] нами было доказано следующее утверждение.

ТеОРема 2.22 [57; следствие 3.2]. Пусть $H$ - матрица Вигнера, распределение отделъных элементов которой имеет гауссово убывание. Тогда для любого $|E|<2$, фиксированного $K u 2<p<\infty$ мъ имеем

$$
\mathrm{P}\left\{\exists \mathbf{v}: H \mathbf{v}=\lambda \mathbf{v},|\lambda-E| \leqslant \frac{K}{N},\|\mathbf{v}\|_{2}=1,\|\mathbf{v}\|_{p} \geqslant Q N^{-1 / 2+1 / p}\right\} \leqslant C e^{-c \sqrt{Q}}
$$

при достаточно больших $Q$ и $N$.

НАБРОСОК ДОКАЗАТЕЛЬСТВА. Мы дадим доказательство более слабого результата, допуская логарифмические множители. Пусть $H \mathbf{v}=\lambda \mathbf{v}$ и $\lambda \in$ $\left[-2+\kappa_{0}, 2-\kappa_{0}\right]$ с $\kappa_{0}>0$. Рассмотрим разложение

$$
H=\left(\begin{array}{cc}
h & \mathbf{a}^{*} \\
\mathbf{a} & H^{(1)}
\end{array}\right)
$$

введенное в п. 2.1, т. е. мы имеем $\mathbf{a}=\left(h_{1,2}, \ldots, h_{1, N}\right)^{*}$ и $H^{(1)}$ - матрица размера $(N-1) \times(N-1)$, получаемая удалением первой строки и первого столбца из матрицы $H$. Пусть $\mu_{\alpha}$ и $\mathbf{u}_{\alpha}$ (при $\alpha=1, \ldots, N-1$ ) обозначают собственные значения и нормированные собственные векторы матрицы $H^{(1)}$. Тогда из уравнения $H \mathbf{v}=\lambda \mathbf{v}$ для собственных значений мы находим

$$
\begin{array}{r}
(h-\lambda) v_{1}+\mathbf{a} \cdot v^{\prime}=0, \\
v_{1} \mathbf{a}+\left(H^{(1)}-\lambda\right) v^{\prime}=0,
\end{array}
$$


где мы представили собственный вектор в виде $\mathbf{v}=\left(v_{1}, v^{\prime}\right), v_{1} \in \mathbb{R}, v^{\prime} \in \mathbb{R}^{N-1}$. Решая второе уравнение относительно $v^{\prime}$, мы получаем $v^{\prime}=v_{1}\left(\lambda-H^{(1)}\right)^{-1} \mathbf{a}$. Тогда из условия нормировки $\|\mathbf{v}\|^{2}=v_{1}^{2}+\left\|v^{\prime}\right\|^{2}=1$ мы получаем следующее соотношение на первую компоненту вектора $\mathbf{v}$ :

$$
\left|v_{1}\right|^{2}=\frac{1}{1+\mathbf{a} \cdot\left(\lambda-H^{(1)}\right)^{-2} \mathbf{a}}=\frac{1}{1+N^{-1} \sum_{\alpha} \xi_{\alpha} /\left(\lambda-\mu_{\alpha}\right)^{2}} \leqslant \frac{4 N \eta^{2}}{\sum_{\mu_{\alpha} \in I} \xi_{\alpha}},
$$

где во втором равенстве мы положили $\xi_{\alpha}=\left|\sqrt{N} \mathbf{a} \cdot \mathbf{u}_{\alpha}\right|^{2}$ и использовали спектральное представление матрицы $H^{(1)}$. Мы также выберем отрезок $I$ длины $\eta=|I|=Q / N$. Легко проверить, что $\mathrm{E} \xi_{\alpha}=1$ и что различные $\xi_{\alpha}$ являются фактически независимыми и удовлетворяют следующей оценке больших уклонений:

$$
\mathrm{P}\left(\sum_{\alpha \in \mathscr{I}} \xi_{\alpha} \leqslant \frac{m}{2}\right) \leqslant e^{-c \sqrt{m}}
$$

где $m=|\mathscr{I}|$ есть мощность множества индексов. Имеется несколько доказательств этого факта, в зависимости от распределения отдельных значений. Например, при условии гауссова убывания данное утверждение было доказано как лемма 4.7 в [57] при помощи теоремы Хансона-Райта [101].

Пусть теперь $\mathscr{N}_{I}$ обозначает число собственных значений матрицы $H$ на отрезке $I$. Из локального полукругового закона (см., например, теорему 1.11) мы знаем, что величина $\mathscr{N}_{I}$ имеет порядок $N|I|$ для любого отрезка $I$ вдали от границ спектра, имеющего длину $|I| \gg 1 / N$. Напомним, что собственные значения матрицы $H$, т. е. $\lambda_{1} \leqslant \cdots \leqslant \lambda_{N}$, и собственные значения матрицы $H^{(1)}$ чередуются. Это означает, что найдется по крайней мере $\mathscr{N}_{I}-1$ собственных значений матрицы $H^{(1)}$ на отрезке $I$. Следовательно, используя одинаковую распределенность компонент любого собственного вектора, мы получаем

$$
\begin{aligned}
& \mathrm{P}\left(\exists \mathbf{v} \text { с } H \mathbf{v}=\lambda \mathbf{v},\|\mathbf{v}\|=1, \lambda \in I \text { и }\|\mathbf{v}\|_{\infty} \geqslant \frac{Q}{N^{1 / 2}}\right) \\
& \leqslant N^{2} \mathrm{P}\left(\exists \mathbf{v} \text { с } H \mathbf{v}=\lambda \mathbf{v},\|\mathbf{v}\|=1, \lambda \in I \text { и }\left|v_{1}\right|^{2} \geqslant \frac{Q^{2}}{N}\right) \\
& \leqslant C N^{2} \mathrm{P}\left(\sum_{\mu_{\alpha} \in I} \xi_{\alpha} \leqslant \frac{4 N^{2} \eta^{2}}{Q^{2}}\right) \\
& \leqslant C N^{2} \mathrm{P}\left(\sum_{\mu_{\alpha} \in I} \xi_{\alpha} \leqslant \frac{4 N^{2} \eta^{2}}{Q^{2}} \text { и } \mathscr{N}_{I} \geqslant c N|I|\right)+C N^{2} \mathrm{P}\left(\mathscr{N}_{I} \leqslant c N|I|\right) \\
& \leqslant C N^{2} e^{-\tilde{c} \sqrt{N|I|}}+C N^{2} e^{-c \sqrt{N|I|}} \leqslant C e^{-c \sqrt{Q}}
\end{aligned}
$$

при условии $4 N^{2} \eta^{2} / Q^{2} \leqslant c N|I|=c N|I|$, т. е. $Q \geqslant \sqrt{N \eta}$.

Здесь мы использовали тот факт, что отклонения от полукругового закона субэкспоненциально подавляются, т. е.

$$
\mathrm{P}\left(\mathscr{N}_{I} \leqslant c N|I|\right) \leqslant e^{-c^{\prime} \sqrt{N|I|}}
$$

для достаточно малых $c$ и $c^{\prime}$, если отрезок $I$ находится вдали от границ спектра. Такая сильная субэкспоненциальная оценка не вытекает непосредственно 
из локального полукругового закона (теоремы 2.5, доказательство которой было намечено в п. 2.3), но может быть доказана для матриц Вигнера с гауссовым убыванием (см. теорему 1.11).

\section{3. Универсальность для гауссовых конволюций}

3.1. Сильная локальная эргодичность броуновского движения Дайсона. В этом пункте мы рассматриваем следующий общий вопрос. Пусть $\mu=$ $e^{-N \mathscr{H}} / Z$ - вероятностная мера на конфигурационном пространстве $\mathbb{R}^{N}$, задаваемая некоторым гамильтонианом $\mathscr{H}: \mathbb{R}^{N} \rightarrow \mathbb{R}$, где $Z=\int e^{-N \mathscr{H}(\mathbf{x})} \mathrm{d} \mathbf{x}<\infty-$ нормировка. Мы всегда будем предполагать, что гамильтониан $\mathscr{H}$ симметричен относительно перестановок переменных $\mathbf{x}=\left(x_{1}, \ldots, x_{N}\right) \in \mathbb{R}^{N}$. Типичным примером является гамильтониан (1.45) для общих $\beta$-ансамблей или частные случаи ГОА $(\beta=1)$ и ГУА $(\beta=2)$.

Рассмотрим зависящую от времени и симметричную относительно перестановок плотность вероятности $f_{t}(\mathbf{x}), t \geqslant 0$, по отношению к мере $\mu(\mathrm{d} \mathbf{x})=$ $\mu(\mathbf{x}) \mathrm{d} \mathbf{x}$, т. е. $\int f_{t}(\mathbf{x}) \mu(\mathrm{d} \mathbf{x})=1$. Динамика задается уравнением эволюции

$$
\partial_{t} f_{t}=L f_{t}, \quad t \geqslant 0
$$

с симметричным относительно перестановок начальным условием $f_{0}$. Порождающий оператор $L$ определяется при помощи формы Дирихле следующим образом:

$$
D(f):=D_{\mu}(f)=-\int f L f \mathrm{~d} \mu=\sum_{j=1}^{N} \frac{1}{2 N} \int\left(\partial_{j} f\right)^{2} \mathrm{~d} \mu, \quad \partial_{j}=\partial_{x_{j}} .
$$

Формально мы имеем $L=\frac{1}{2 N} \Delta-\frac{1}{2}(\nabla \mathscr{H}) \nabla$. Мы не будем рассматривать вопросы, связанные с областью определения. Заметим лишь, что $D(f)$ является полуограниченной квадратичной формой, так что $L$ может быть определен при помощи продолжения Фридрихса на $L^{2}(\mathrm{~d} \mu)$, и он может быть также продолжен на $L^{1}$. Динамика определена корректно для любого начального условия $f_{0} \in$ $L^{1}(\mathrm{~d} \mu)$. Подробности можно найти в приложении А к работе [6].

Строго говоря, мы будем рассматривать последовательность гамильтонианов $\mathscr{H}_{N}$ и соответствующие динамики $L_{N}$ и $f_{t, N}$, параметризованные числом $N$, но зависимость от $N$ будет игнорироваться. Все результаты будут относиться к пределу при $N \rightarrow \infty$.

Наряду с (3.1) можно описывать динамику спаренной системы стохастических дифференциальных уравнений (1.64), как было указано в п. 1.6.2, но мы не будем здесь использовать этот формализм.

Для любого $k \geqslant 1$ определим $k$-точечные корреляционные функции (маргиналы) вероятностной меры $f_{t} \mathrm{~d} \mu$ как

$$
p_{t, N}^{(k)}\left(x_{1}, \ldots, x_{k}\right)=\int_{\mathbb{R}^{N-k}} f_{t}(\mathbf{x}) \mu(\mathbf{x}) \mathrm{d} x_{k+1} \cdots \mathrm{d} x_{N} .
$$


Корреляционные функции равновесной меры обозначаются через

$$
p_{\mu, N}^{(k)}\left(x_{1}, \ldots, x_{k}\right)=\int_{\mathbb{R}^{N-k}} \mu(\mathbf{x}) \mathrm{d} x_{k+1} \cdots \mathrm{d} x_{N} .
$$

Теперь мы перечислим наши основные условия на начальное распределение $f_{0}$ и на его эволюцию $f_{t}$. Этот формализм предназначен для обобщенных матриц Вигнера; случайные ковариационные матрицы потребуют некоторых модификаций (подробности см. в [6]). Вначале определим подобласть

$$
\Sigma_{N}:=\left\{\mathbf{x} \in \mathbb{R}^{N}: x_{1}<x_{2}<\cdots<x_{N}\right\}
$$

упорядоченных наборов точек $\mathbf{x}$.

УСловиЕ І. Гамильтониан $\mathscr{H}$ равновесной меры имеет вид

$$
\mathscr{H}=\mathscr{H}_{N}(\mathbf{x})=\beta\left[\sum_{j=1}^{N} U\left(x_{j}\right)-\frac{1}{N} \sum_{i<j} \log \left|x_{i}-x_{j}\right|\right],
$$

где $\beta \geqslant 1$. Функция $U: \mathbb{R} \rightarrow \mathbb{R}$ является гладкой, причем $U^{\prime \prime} \geqslant 0$ и

$$
U(x) \geqslant C|x|^{\delta} \quad \text { для некоторого } \delta>0 \text { и больиих }|x| \text {. }
$$

Заметим, что это условие выполняется автоматически для симметрических и эрмитовых матриц Вигнера, а инвариантной мерой является ГОА и ГУА соответственно (см. (1.44)-(1.45)).

Вблизи компоненты границы множества $\Sigma_{N}$, которая выделяется условием $x_{i+1}=x_{i}$, порождающий оператор имеет вид

$$
L=\frac{1}{4 N}\left(\partial_{u}^{2}+\frac{\beta}{u} \partial_{u}\right)+\text { регулярный оператор }
$$

в относительных координатах $u=\left(x_{i+1}-x_{i}\right) / 2$ при $u \ll 1$. Известно (см. [6; приложение $\mathrm{A}]$ ), что при $\beta \geqslant 1$ отталкивающая сила сингулярной диффузии достаточно сильна, чтобы предотвратить падение частиц в начало координат. Это соответствует тому, что в исходных координатах траектории точек не пересекаются. В частности, порядок точек сохраняется под действием динамики (стохастическое доказательство этого факта дано в лемме 4.3 .3 из [3]). Таким образом, в дальнейшем мы можем предполагать, что $f_{t}$ является вероятностной мерой на $\Sigma_{N}$. Мы будем продолжать использовать обозначения $f$ и $\mu$ для мер, получаемых ограничением на $\Sigma_{N}$. Заметим, что корреляционные функции $p^{(k)}$ из (3.3) по-прежнему определены в $\mathbb{R}^{k}$, т. е. их аргументы остаются неупорядоченными.

Из условия I следует, что матрица Гессе гамильтониана $\mathscr{H}$ удовлетворяет следующей оценке:

$$
\left\langle\mathbf{v}, \nabla^{2} \mathscr{H}(\mathbf{x}) \mathbf{v}\right\rangle \geqslant \frac{\beta}{N} \sum_{i<j} \frac{\left(v_{i}-v_{j}\right)^{2}}{\left(x_{i}-x_{j}\right)^{2}}, \quad \mathbf{v}=\left(v_{1}, \ldots, v_{N}\right) \in \mathbb{R}^{N}, \quad \mathbf{x} \in \Sigma_{N}
$$

Эта выпуклая оценка является ключевым предположением; наш метод работает для широкого класса общих гамильтонианов при условии выполнения 
неравенства (3.6). В частности, к гамильтониану (3.5) можно добавить функцию потенциала $V(\mathbf{x})$ произвольного числа тел, при условии, что эта функция $V$ выпукла в области $\Sigma_{N}$.

УСловие II. Существует непрерывная функция плотности $\varrho(x) \geqslant 0$, $\int_{\mathbb{R}} \varrho=1$, с компактным носителем на вещественной оси, не зависящая от $N$, такая, что для любых фиксированных $a, b \in \mathbb{R}$ выполнено равенство

$$
\lim _{N \rightarrow \infty} \sup _{t \geqslant 0}\left|\int \frac{1}{N} \sum_{j=1}^{N} \mathbf{1}\left(x_{j} \in[a, b]\right) f_{t}(\mathbf{x}) \mathrm{d} \mu(\mathbf{x})-\int_{a}^{b} \varrho(x) \mathrm{d} x\right|=0 .
$$

Другими словами, мы предполагаем, что предельная плотность существует; для матриц Вигнера это в точности полукруговой закон. Пусть $\gamma_{j}=\gamma_{j, N}$ обозначает расположение $j$-й точки для предельной плотности, т. е. $\gamma_{j}$ определяется формулой

$$
N \int_{-\infty}^{\gamma_{j}} \varrho(x) \mathrm{d} x=j, \quad 1 \leqslant j \leqslant N, \quad \gamma_{j} \in \operatorname{supp} \varrho .
$$

Мы будем называть $\gamma_{j}$ классическим расположением $j$-й точки. Заметим, что $\gamma_{j}$ может быть определено неоднозначно, если носитель плотности $\varrho$ не связен, но в этом случае не будет выполнено следующее условие III.

УСловие III. Существует число $\mathfrak{a}>0$, для которого имеет место неравенство

$$
\sup _{t \geqslant N^{-2 \mathfrak{a}}} \int \frac{1}{N} \sum_{j=1}^{N}\left(x_{j}-\gamma_{j}\right)^{2} f_{t}(\mathrm{~d} \mathbf{x}) \mu(\mathrm{d} \mathbf{x}) \leqslant C N^{-1-2 \mathfrak{a}}
$$

с некоторой константой $C$ и равномерно по $N$.

При выполнении условия II типичное расстояние между соседними точками имеет порядок $1 / N$ вдали от границ спектра, т. е. в окрестности любой энергии $E$ с $\varrho(E)>0$. Условие III гарантирует, что случайные точки $x_{j}$, как правило, находятся в $N^{-1 / 2-\mathfrak{a}}$-окрестности их классических расположений.

Последнее условие представляет собой верхнюю границу на локальную плотность. Для любого отрезка $I \subset \mathbb{R}$ пусть $\mathscr{N}_{I}:=\sum_{i=1}^{N} \mathbf{1}\left(x_{i} \in I\right)$ обозначает число точек внутри $I$.

УСловие IV. Для любого отрезка $I_{0} \subset\{E: \varrho(E)>0\}$ и для любъх $\delta>0$, $\sigma>0$ существуют константы $C_{n}, n \in \mathbb{N}$, зависящие от $I_{0}, \delta$ u $\sigma$, такие, что для любого отрезка $I \subset I_{0} c|I| \geqslant N^{-1+\sigma}$ и для любого $K \geqslant 1$ мы имеем

$$
\sup _{\tau \geqslant N^{-2 a}} \int \mathbf{1}\left\{\mathscr{N}_{I} \geqslant K N|I|\right\} f_{\tau} \mathrm{d} \mu \leqslant C_{n} K^{-n}, \quad n=1,2, \ldots
$$

где $\mathfrak{a}$ - показатель из условия III. 
Заметим, что для симметрических или эрмитовых матриц Вигнера условие I выполнено автоматически, условие II есть (глобальный) полукруговой закон (1.27), а условие IV есть верхняя граница на плотность из леммы 1.10. Единственным существенным условием является неравенство (3.9).

Следующая общая теорема утверждает, что локальные статистики точек $x_{j}$ внутри спектра по отношению к эволюционирующему со временем распределению $f_{t}$ совпадают с локальными статистиками по отношению к равновесной мере $\mu$, при условии $t \gg N^{-2 \mathfrak{a}}$.

Теорема 3.1 [6; теорема 2.1]. Предположим, что гамильтониан, задаваемый формулой (3.5), удовлетворяет условию I, а условия II, III и IV выполнены для решения $f_{t}$ уравнения эволюиии (3.1) с показателем а. Пусть в момент времени $t_{0}=N^{-2 \mathfrak{a}}$ плотность $f_{t_{0}}$ удовлетворяет условию ограниченной энmponuu, m.e.

$$
S_{\mu}\left(f_{t_{0}}\right):=\int f_{t_{0}} \log f_{t_{0}} \mathrm{~d} \mu \leqslant C N^{m}
$$

для некоторого фиксированного показателя $m$. Пусть $E \in \mathbb{R} u b>0$ удовлетворяют условию $\min \{\varrho(x): x \in[E-b, E+b]\}>0$. Тогда для любого $\delta>0$, для любого иелого $k \geqslant 1$ и для любой тестовой функиии $O: \mathbb{R}^{k} \rightarrow \mathbb{R}$ с компактным носителем мы имеем, для $\tau:=N^{-2 \mathfrak{a}+\delta}$,

$$
\begin{aligned}
\lim _{N \rightarrow \infty} & \sup _{t \geqslant \tau} \int_{E-b}^{E+b} \frac{\mathrm{d} E^{\prime}}{2 b} \int_{\mathbb{R}^{k}} \mathrm{~d} \alpha_{1} \cdots \mathrm{d} \alpha_{k} O\left(\alpha_{1}, \ldots, \alpha_{k}\right) \\
& \times \frac{1}{\varrho(E)^{k}}\left(p_{t, N}^{(k)}-p_{\mu, N}^{(k)}\right)\left(E^{\prime}+\frac{\alpha_{1}}{N \varrho(E)}, \ldots, E^{\prime}+\frac{\alpha_{k}}{N \varrho(E)}\right)=0 .
\end{aligned}
$$

Заметим, что данный предел можно эффективно контролировать: например, в [6] нами доказано, что до перехода к пределу при $N \rightarrow \infty$ предел левой части соотношения (3.12) ограничен величиной $C N^{2 \varepsilon^{\prime}}\left[b^{-1} N^{-(1+2 \mathfrak{a}) / 3}+\right.$ $\left.b^{-1 / 2} N^{-\delta / 2}\right]$.

Во многих приложениях локальные равновесные статистики могут быть явно вычислены и в пределе при $b \rightarrow 0$ становятся независимыми от $E$. В частности, это имеет место для классических матричных ансамблей. Наиболее простая явная формула получается для случая ГУА, когда корреляционные функции задаются синус-ядром (1.35).

3.2. Локальный релаксационный поток. Доказательство теоремы 3.1 опиралось на анализ прихода в равновесие (релаксации) динамики (3.1). Равновесие задается гамильтонианом $\mathscr{H}$, который близок к выпуклому, так что применим метод Бакри-Эмери [91]. Этот подход был впервые применен к броуновскому движению Дайсона в [89; раздел 5.1]; в нашем изложении здесь мы следуем [6].

Чтобы дать представление о методе, предположим временно, что потенциал $U$ в (3.5) является равномерно выпуклым, т. е.

$$
U^{\prime \prime}(x) \geqslant U_{0}>0 \text {. }
$$

Это всегда имеет место для гауссовых ансамблей, когда $U(x)=x^{2} / 4$. В этом случае мы имеем следующую нижнюю оценку на гессиан функции $\mathscr{H}$ :

$$
\text { Hess } \mathscr{H} \geqslant \beta U_{0}
$$


на множестве $\Sigma_{N}(3.4)$, так как логарифмический потенциал является выпуклым. Здесь важно отметить, что неравенство (3.13) имеет место лишь на открытом множестве $\Sigma_{N}$, так как вторые производные логарифмических взаимодействий имеют особенность вида дельта-функции (с “неправильным" знаком) на границе. Требуется дополнительное техническое рассуждение для того, чтобы показать, что при $\beta \geqslant 1$ точки отталкиваются достаточно сильно, так что броуновское движение Дайсона никогда не покидает открытого множества $\Sigma_{N}$, и, следовательно, применим метод Бакри-Эмери. См. замечание после теоремы 3.2 .

Следующий методический раздел посвящен общей постановке критерия Бакри-Эмери в $\mathbb{R}^{N}$.

3.2.1. Метод Бакри-Эмери. Пусть вероятностная мера $\mu$ в $\mathbb{R}^{N}$ задается строго выпуклым гамильтонианом $\mathscr{H}$ :

$$
\mathrm{d} \mu(\mathbf{x})=\frac{e^{-\mathscr{H}(\mathbf{x})}}{Z} \mathrm{~d} \mathbf{x}, \quad \nabla^{2} \mathscr{H}(\mathbf{x})=\operatorname{Hess} \mathscr{H}(\mathbf{x}) \geqslant K>0
$$

с некоторой константой $K$, и пусть $L$ - порождающий оператор динамики, ассоциированной с формой Дирихле

$$
D(f)=D_{\mu}(f)=-\int f L f \mathrm{~d} \mu:=\frac{1}{2} \sum_{j} \int\left(\partial_{j} f\right)^{2} \mathrm{~d} \mu, \quad \partial_{j}=\partial_{x_{j}}
$$

(заметим, что в данном изложении мы игнорируем множитель $1 / N$, который изначально присутствовал в (3.2)). Формально мы имеем $L=\frac{1}{2} \Delta-\frac{1}{2}(\nabla \mathscr{H}) \nabla$. Оператор $L$ симметричен по отношению к мере $\mathrm{d} \mu$, т. е.

$$
\int f L g \mathrm{~d} \mu=\int(L f) g \mathrm{~d} \mu=-\frac{1}{2} \int \nabla f \cdot \nabla g \mathrm{~d} \mu .
$$

Мы определим относительную энтропию произвольной плотности вероятности $f$ с $\int f \mathrm{~d} \mu=1$ по формуле

$$
S_{\mu}(f)=S(f)=\int f(\log f) \mathrm{d} \mu .
$$

Как форма Дирихле, так и энтропия являются неотрицательными, обращаются в нуль только при $f \equiv 1$ и измеряют расстояние от $f$ до положения равновесия $f=1$. Энтропию можно использовать для непосредственного контроля над полной вариационной нормой при помощи неравенства энтропии

$$
\int|f-1| \mathrm{d} \mu \leqslant \sqrt{2 S_{\mu}(f)} .
$$

Пусть $f_{t}$ - решение уравнения эволюции

$$
\partial_{t} f_{t}=L f_{t}, \quad t>0
$$


с данным начальным условием $f_{0}$; рассмотрим эволюцию энтропии $S\left(f_{t}\right)$ и формы Дирихле $D\left(\sqrt{f_{t}}\right)$. Простые вычисления показывают, что

$$
\partial_{t} S\left(f_{t}\right)=\int\left(L f_{t}\right) \log f_{t} \mathrm{~d} \mu+\int f_{t} \frac{L f_{t}}{f_{t}} \mathrm{~d} \mu=-\frac{1}{2} \int \frac{\left(\nabla f_{t}\right)^{2}}{f_{t}} \mathrm{~d} \mu=-4 D\left(\sqrt{f_{t}}\right),
$$

где мы использовали равенство $\int L f_{t} \mathrm{~d} \mu=0$ в силу (3.15). Аналогично можно вычислить эволюцию формы Дирихле. Пусть, для простоты, $h:=\sqrt{f}$, тогда

$$
\partial_{t} h_{t}=\frac{1}{2 h_{t}} \partial h_{t}^{2}=\frac{1}{2 h_{t}} L h_{t}^{2}=L h_{t}+\frac{1}{2 h_{t}}\left(\nabla h_{t}\right)^{2} .
$$

На последнем шаге мы использовали равенство $L h^{2}=(\nabla h)^{2}+2 h L h$, которое можно вывести либо непосредственно из $L=\frac{1}{2} \Delta-\frac{1}{2}(\nabla \mathscr{H}) \nabla$, либо из следующего тождества для любой тестовой функции $g$ :

$$
\begin{aligned}
\int g L h^{2} \mathrm{~d} \mu & =-\frac{1}{2} \int \nabla g \cdot \nabla\left(h^{2}\right) \mathrm{d} \mu=-\int h(\nabla g)(\nabla h) \mathrm{d} \mu \\
& =\int[-\nabla(h g)+g \nabla h] \nabla h \mathrm{~d} \mu=\int g\left[(\nabla h)^{2}+2 h L h\right] \mathrm{d} \mu .
\end{aligned}
$$

Мы имеем (нижний индекс $t$ опущен для краткости):

$$
\begin{aligned}
\partial_{t} D\left(\sqrt{f_{t}}\right)= & \frac{1}{2} \partial_{t} \int(\nabla h)^{2} \mathrm{~d} \mu=\int(\nabla h)(\nabla L h) \mathrm{d} \mu+\frac{1}{2} \int(\nabla h) \cdot \nabla \frac{(\nabla h)^{2}}{h} \mathrm{~d} \mu \\
= & \int(\nabla h)[\nabla, L] h \mathrm{~d} \mu+\int(\nabla h) L(\nabla h) \mathrm{d} \mu \\
& \quad+\frac{1}{2} \int \sum_{i, j} \partial_{i} h\left[\frac{2\left(\partial_{j} h\right) \partial_{i} \partial_{j} h}{h}-\frac{\left(\partial_{j} h\right)^{2} \partial_{i} h}{h^{2}}\right] \mathrm{d} \mu \\
= & -\frac{1}{2} \int(\nabla h)\left(\nabla^{2} \mathscr{H}\right) \nabla h \mathrm{~d} \mu-\frac{1}{2} \int \sum_{i, j}\left(\partial_{i} \partial_{j} h\right)^{2} \mathrm{~d} \mu \\
& \quad+\frac{1}{2} \int \sum_{i, j}\left[\frac{2\left(\partial_{j} h\right)\left(\partial_{i} h\right) \partial_{i j} h}{h}-\frac{\left(\partial_{j} h\right)^{2}\left(\partial_{i} h\right)^{2}}{h^{2}}\right] \mathrm{d} \mu \\
=- & \frac{1}{2} \int(\nabla h)\left(\nabla^{2} \mathscr{H}\right) \nabla h \mathrm{~d} \mu-\frac{1}{2} \int \sum_{i, j}\left(\partial_{i j} h-\frac{\left(\partial_{i} h\right)\left(\partial_{j} h\right)}{h}\right)^{2} \mathrm{~d} \mu
\end{aligned}
$$

где мы использовали коммутатор

$$
[\nabla, L]=-\frac{1}{2}\left(\nabla^{2} \mathscr{H}\right) \nabla
$$

Следовательно, при выполнении условия выпуклости (3.14) мы имеем

$$
\partial_{t} D\left(\sqrt{f_{t}}\right) \leqslant-K D\left(\sqrt{f_{t}}\right)
$$


Из (3.18) и (3.20) получаем

$$
\partial_{t} D\left(\sqrt{f_{t}}\right) \leqslant \frac{K}{4} \partial_{t} S\left(f_{t}\right)
$$

При $t=\infty$ достигается равновесное состояние $f_{\infty}=1$, и энтропия и форма Дирихле становятся нулевыми. Интегрируя неравенство (3.21) в обратном порядке от $t=\infty$, мы получаем логарифмическое неравенство Соболева

$$
S\left(f_{t}\right) \leqslant \frac{4}{K} D\left(\sqrt{f_{t}}\right)
$$

для любого $t \geqslant 0$; в частности, оно имеет место для начального распределения $f=f_{0}$. Подставляя это в $(3.18)$, мы получаем

$$
\partial_{t} S\left(f_{t}\right) \leqslant-K S\left(f_{t}\right)
$$

Интегрируя по времени от нуля, мы получаем экспоненииалъную релаксацию энтропии в масштабе времени $t \sim 1 / K$ :

$$
S\left(f_{t}\right) \leqslant e^{-t K} S\left(f_{0}\right)
$$

Наконец, мы можем проинтегрировать соотношение (3.18) по времени от $t / 2$ до $t$, получив

$$
S\left(f_{t}\right)-S\left(f_{t / 2}\right)=-4 \int_{t / 2}^{t} D\left(\sqrt{f_{\tau}}\right) \mathrm{d} \tau .
$$

Используя положительность энтропии $S\left(f_{t}\right) \geqslant 0$ в левой части и монотонность формы Дирихле (см. (3.20)) в правой части, мы получаем

$$
D\left(\sqrt{f_{t}}\right) \leqslant \frac{2}{t} S\left(f_{t / 2}\right)
$$

Отсюда, используя (3.23), мы получаем экспоненииальную релаксацию формы Дирихле в масштабе времени $t \sim 1 / K$ :

$$
D\left(\sqrt{f_{t}}\right) \leqslant \frac{2}{t} e^{-t K / 2} S\left(f_{0}\right) .
$$

Результаты этих вычислений сведены вместе в следующем утверждении.

ТЕОРема 3.2 [91]. При условии выпуклой оценки на гамилътониан, т.е. $\nabla^{2} \mathscr{H} \geqslant K$ для некоторой положительной константы $K$, мера $\mu=e^{-\mathscr{H}} / Z$ удовлетворяет логарифмическому неравенству Соболева

$$
S(f) \leqslant \frac{4}{K} D(\sqrt{f}) \quad \text { для любой плотности } f \text { с } \int f \mathrm{~d} \mu=1,
$$

а динамика (3.17) приходит в равновесие за время порядка $t \sim 1 / K$ как в смьсле энтропии, так и в смысле формы Дирихле:

$$
S\left(f_{t}\right) \leqslant e^{-t K} S\left(f_{0}\right), \quad D\left(\sqrt{f_{t}}\right) \leqslant \frac{2}{t} e^{-t K / 2} S\left(f_{0}\right) .
$$


ТЕХническое ЗАмЕЧАниЕ. В наших приложениях динамика будет ограничена на подмножество $\Sigma_{N}=\left\{\mathbf{x}: x_{1}<\cdots<x_{N}\right\}$, и поэтому нам необходимо проверить, что граничный член в интегрировании по частям (в третьей и четвертой строках в (3.19)) обращается в нуль, т. е.

$$
\int_{\partial \Sigma} \partial_{i} h \partial_{i j}^{2} h e^{-\mathscr{H}} \mathrm{d} \mathbf{x}=0 .
$$

Роль гамильтониана $\mathscr{H}$ будет играть $N \mathscr{H}_{N}$, где $\mathscr{H}_{N}$ определено в (3.5). Хотя функция плотности меры $e^{-N \mathscr{H}_{N}}$ ведет себя как $\left(x_{i+1}-x_{i}\right)^{\beta}$ вблизи компоненты границы, выделяемой соотношением $x_{i+1}=x_{i}$, а значит, обращается в нуль на границе, функция $f_{t}$ является решением параболического уравнения с особым членом, так что в принципе она может претерпевать особенность на границе. Другая сложность связана с тем, что $h=\sqrt{f}$, а производная квадратного корня имеет особенность. Тем не менее, применяя теорию параболической регулярности и функции срезки, должным образом учитывающие геометрию множества $\Sigma_{N}$, можно доказать, что интеграл (3.27) обращается в нуль. Это является одной из причин, по которым необходимо ограничение $\beta \geqslant 1$. Подробности можно найти в [6; приложение В].

3.2.2. Универсальность распределения промежутков для броуновского движения Дайсона за короткое время. При использовании выпуклой оценки (3.13) для броуновского движения Дайсона метод Бакри-Эмери показывает, что мера $\mu$ удовлетворяет логарифмическому неравенству Соболева, а время прихода в равновесие имеет порядок один.

Следующий результат является основной теоремой п. 3.2. Он показывает, что время прихода в равновесие в действительности намного меньше, чем порядка один, по крайней мере локально и для наблюдаемых, которые зависят только от разностей собственных значений.

Теорема 3.3 (универсальность броуновского движения Дайсона за короткое время [6; теорема 4.1]). Предположим, что гамильтониан, задаваемый формулой (3.5), удовлетворяет выпуклой оценке (3.6) с $\beta \geqslant 1$. Пусть $f_{t}-$ pешение уравнения эволюиии (3.1), т.е. после прохождения времени $t_{0}=N^{-2 \mathfrak{a}}$ оно удовлетворяет неравенству $S_{\mu}\left(f_{t_{0}}\right):=\int f_{t_{0}}\left(\log f_{t_{0}}\right) \mathrm{d} \mu \leqslant C N^{m}$ для некоторого фиксированного $\mathrm{m}$. Положим

$$
Q:=\sup _{t \geqslant t_{0}} \sum_{j} \int\left(x_{j}-\gamma_{j}\right)^{2} f_{t} \mathrm{~d} \mu
$$

и предположим, что $Q \leqslant C N^{m}$ с некоторым показателем $m$. Зафиксируем $n \geqslant 1$ и набор возрастающих положительных иельх чисел $\mathbf{m}=\left(m_{1}, \ldots, m_{n}\right) \in$ $\mathbb{N}_{+}^{n}$. Пусть $G: \mathbb{R}^{n} \rightarrow \mathbb{R}$ - ограниченная гладкая функиия с компактным носителем; положим

$$
\mathscr{G}_{i, \mathbf{m}}(\mathbf{x}):=G\left(N\left(x_{i}-x_{i+m_{1}}\right), N\left(x_{i+m_{1}}-x_{i+m_{2}}\right), \ldots, N\left(x_{i+m_{n-1}}-x_{i+m_{n}}\right)\right) .
$$

Тогда для любого достаточно малого $\varepsilon^{\prime}>0$ существуют константы $C, c>0$, зависящие только от $\varepsilon^{\prime} u G$, такие, что для любого $J \subset\left\{1,2, \ldots, N-m_{n}\right\}$ 
и любого $\tau>3 t_{0}=3 N^{-2 \mathfrak{a}}$ мъ имеем

$\left|\int \frac{1}{N} \sum_{i \in J} \mathscr{G}_{i, \mathbf{m}}(\mathbf{x}) f_{\tau} \mathrm{d} \mu-\int \frac{1}{N} \sum_{i \in J} \mathscr{G}_{i, \mathbf{m}}(\mathbf{x}) \mathrm{d} \mu\right| \leqslant C N^{\varepsilon^{\prime}} \sqrt{|J| Q(N \tau)^{-1}}+C e^{-c N^{\varepsilon^{\prime}}}$.

В п. 3.2.3 мы приведем идею доказательства. Полное доказательство будет дано в п. 3.2.4.

3.2.3. Интуитивное доказательство теоремы 3.3. Ключевая идея заключается в том, что приход в локальное равновесие можно “ускорить", модифицируя динамику путем добавления некоторого дополнительного потенциала $W(\mathbf{x})$ к гамильтониану. Он будет иметь вид

$$
W(\mathbf{x}):=\sum_{j=1}^{N} W_{j}\left(x_{j}\right), \quad W_{j}(x):=\frac{1}{2 R^{2}}\left(x_{j}-\gamma_{j}\right)^{2},
$$

т. е. это - квадратичный потенциал порядка $R$, заключающий каждое собственное значение вблизи его классического расположения. Мы определим

$$
\widetilde{\mathscr{H}}:=\mathscr{H}+W \text {. }
$$

Новая мера будет обозначаться следующим образом:

$$
\mathrm{d} \omega:=\omega(\mathbf{x}) \mathrm{d} \mathbf{x}, \quad \omega:=e^{-N \widetilde{\mathscr{H}}} / \widetilde{Z},
$$

и будет называться псевдоравновесной мерой (для упрощения обозначений мы будем обозначать через $\omega$ как меру, так и ее функцию плотности по отношению к мере Лебега). Соответствующий порождающий оператор будет обозначаться через $\widetilde{L}$. Мы, как правило, будем выбирать $R \ll 1$, так что добавочный член $W$ будет существенно увеличивать нижнюю оценку (3.13) на гессиан, тем самым ускоряя время прихода динамики в равновесие от порядка $O(1)$ до порядка $O\left(R^{2}\right)$. Это соображение будет первым шагом доказательства и будет сформулировано в виде теоремы 3.4, доказательство которой будет следовать методу Бакри-Эмери из п. 3.2.1.

На втором шаге мы рассматриваем произвольную вероятностную меру вида $q \omega$ для некоторой функции $q$ и контролируем разности значений математического ожидания

$$
\int \mathbf{G} q \mathrm{~d} \omega-\int \mathbf{G} \mathrm{d} \omega
$$

наблюдаемых

$$
\mathbf{G}:=\frac{1}{N} \sum_{i \in J} \mathscr{G}_{i, \mathbf{m}}
$$

в терминах энтропии и формы Дирихле функции $q$ по отношению к мере $\omega$. Это позволит нам сравнить математические ожидания

$$
\int \mathbf{G} q \mathrm{~d} \omega-\int \mathbf{G} q^{\prime} \mathrm{d} \omega
$$


для любых двух мер $q \omega$ и $q^{\prime} \omega$, в частности, для мер $f_{\tau} \mu$ и $\mu$, которые можно представить в этом виде, положив $q=f_{\tau} \mu / \omega$ и $q^{\prime}=\mu / \omega$.

Здесь мы сталкиваемся с $N$-проблемой: как энтропия, так и форма Дирихле являются большими величинами. Непосредственное применение неравенства энтропии (3.16) к (3.33) даст оценку величины порядка один (наблюдаемой $\mathbf{G}$ ) величиной порядка $O(\sqrt{N})$. Вместо этого мы можем запустить новую динамику до некоторого времени $\tau$ и записать

$$
\int \mathbf{G} q \mathrm{~d} \omega-\int \mathbf{G} \mathrm{d} \omega=\int \mathbf{G}\left(q-q_{\tau}\right) \mathrm{d} \omega+\int \mathbf{G}\left(q_{\tau}-1\right) \mathrm{d} \omega .
$$

Если $\tau$ больше, чем время релаксации новой динамики, то второй член является экспоненциально малым в силу неравенства энтропии, и эта экспоненциальная малость подавляет $N$-проблему.

Для того чтобы оценить первый член, нам нужно будет воспользоваться тем, что $\tau$ мало. Путем обратного интегрирования производной по времени, $q_{\tau}-q=\int_{0}^{\tau} \partial_{t} q_{t} \mathrm{~d} t$, мы можем извлечь множитель, пропорциональный $\tau$. Однако после использования соотношения $\partial q_{t}=\widetilde{L} q_{t}$ и интегрирования по частям нам придется дифференцировать наблюдаемую, что даст дополнительный множитель $N$ из-за ее порядка. Может показаться, что этот метод дает оценку величины порядка один величиной порядка $N$. В действительности же мы доказываем новую оценку (см. (3.45) ниже), которая позволяет контролировать величину $\int \mathbf{G}\left(q-q_{\tau}\right) \mathrm{d} \omega$ при помощи $\left(\tau D_{\omega}(\sqrt{q}) / N\right)^{1 / 2}$; отметим здесь дополнительный множитель $1 / N$. Главной причиной этого улучшения является тот факт, что в некоторых направлениях, а именно, для наблюдаемых, зависящих лищъ от разностей переменных $x_{i}$, динамика приходит в равновесие намного быстрее. Чтобы извлечь этот механизм, мы воспользуемся тем фактом, что нижняя оценка (3.6) на гессиан имеет порядок $N$ относительно разностных переменных $v_{i}-v_{j}$, и эта оценка может быть использована для получения дополнительного $N$-множителя. Это утверждение содержится в теореме 3.5. Данная оценка будет иметь свободный параметр $\tau$, который может быть оптимизирован. Этот параметр происходит из способа доказательства: мы доказываем неравенство, не зависящее от времени, динамическим методом, т. е. мы рассматриваем эволюцию потока до некоторого времени $\tau$ и оцениваем разности $q-q_{\tau}$ и $q_{\tau}-q_{\infty}$ различными способами.

Наконец, на третьем шаге мы должны сравнить исходную динамику с новой в смысле энтропии и формы Дирихле, ввиду того, что для оценок на втором шаге нам необходимо подсчитать величины $D_{\omega}\left(\sqrt{f_{\tau} \mu / \omega}\right)$ и $S_{\omega}\left(f_{\tau} \mu / \omega\right)$. Это можно было бы проделать при помощи стандартного сведения к равновесным оценкам (3.26), если бы величина $f_{\tau} \mu / \omega$ эволюционировала под действием модифицированной динамики, но $f_{\tau}$ эволюционирует под действием исходной динамики. Поэтому нам необходимо показать, что погрешность, возникающая при модификации динамики путем добавления члена $W$, несущественна.

Оказывается, что ключевой величиной, определяющей погрешность, возникающую при введении дополнительного потенциала, является $H^{1}$-норма функ- 
ции $W$, т. е.

$$
\Lambda_{t}:=\int(\nabla W)^{2} f_{t} \mathrm{~d} \mu .
$$

Так как $W$ задается явно, мы имеем

$$
\Lambda_{t}=R^{-4} \sum_{i} \int\left(x_{i}-\gamma_{i}\right)^{2} f_{t} \mathrm{~d} \mu \leqslant C N^{-2 \mathfrak{a}} R^{-4},
$$

где мы воспользовались условием III (см. (3.9)). Для данного $\mathfrak{a}>0$ мы тем самым можем выбрать $R \ll 1$ таким образом, что по-прежнему имеет место неравенство $\Lambda \ll 1$. Это и будет завершением доказательства.

Заметим, что скорость сходимости определяется второй производной дополнительного потенциала, в то время как изменение формы Дирихле и энтропии определяется величиной $(\nabla W)^{2}$. Поэтому мы можем ускорить динамику, при этом по-прежнему контролируя формы Дирихле и энтропии двух равновесных мер, если имеет место сильная априорная оценка (3.34) на $\Lambda$. Это является одной из причин, по которым данный метод работает.

Принципиальное значение здесь имеет эффективное использование выпуклой оценки (3.6), которое опирается на ключевое свойство динамики броуновского движения Дайсона (1.64). Потенциал логарифмического взаимодействия приводит к сингулярной силе

$$
F\left(x_{i}\right)=-\frac{1}{4} x_{i}-\frac{1}{2 N} \sum_{j \neq i} \frac{1}{x_{i}-x_{j}},
$$

действующей на $i$-ю частицу. Формально, $F\left(x_{i}\right)$ является силой среднего поля, и если бы $x_{j}$ были распределены в соответствии с полукруговым законом, то основная часть суммы сократилась бы с членом $-x_{i} / 4$. Однако соседние частицы $(j=i \pm 1)$ оказывают огромный эффект: они действуют с силой порядка один на $x_{i}$. Такая сила может сдвинуть частицу $x_{i}$ на расстояние порядка $1 / N$ за очень короткое время порядка $1 / N$. Заметим, что в силу свойства сохранения порядка под действием динамики, отрезок размера $O(1 / N)$ является примерно всем доступным пространством для частицы $x_{i}$, по крайней мере для основной массы частиц. Следовательно, частица $x_{i}$ с большой вероятностью придет в равновесие за время порядка $1 / N$, ввиду наличия сильной отталкивающей силы со стороны ее соседей. Конечно, это рассуждение не является доказательством, так как другие частицы также движутся. Тем не менее, наши наблюдаемые зависят лишь от разностей собственных значений, а в разностных координатах сильное движение присутствует постоянно. Это показывает, что разности собственных значений могут придти в локальное равновесие за время порядка почти $1 / N$.

Что касается других наблюдаемых, их время релаксации непременно длиннее. В частности, может случиться так, что основная часть суммы в (3.35) не будет приводить к точным сокращениям, что соответствует тому, что все соседние частицы испытывают одинаковое влияние среднего поля и двигаются совместно. В действительности, если изначальный вид плотности существенно отличается от полукруга, то релаксация к полукруговому закону может происходить даже за время порядка один (хотя такой сценарий исключается в нашем случае ввиду условия (3.9)). 
3.2.4. Подробное доказательство теоремы 3.3. Все константы в этом доказательстве зависят от $\varepsilon^{\prime}$ и $G$, и мы не будем указывать явную зависимость. Для данного $\tau>0$ определим $R:=\tau^{1 / 2} N^{-\varepsilon^{\prime} / 2}$.

Введем псевдоравновесную меру $\omega_{N}=\omega=\psi \mu$ формулой

$$
\psi:=\frac{Z}{\widetilde{Z}} \exp (-N W)
$$

где $\widetilde{Z}$ выбрано таким образом, что $\omega$ является вероятностной мерой, в частности, $\omega=e^{-N \widetilde{\mathscr{H}}} / \widetilde{Z} \mathrm{c}$

$$
\widetilde{\mathscr{H}}=\mathscr{H}+W .
$$

Потенциал $W$ задается формулой $(3.31)$, и он заключает $j$-ю точку $x_{j}$ вблизи ее классического расположения $\gamma_{j}$.

Локальный релаксационный поток определяется как обратимая динамика по отношению к мере $\omega$. Эта динамика описывается при помощи порождающего оператора $\widetilde{L}$, определяемого формулой

$$
\int f \widetilde{L} g \mathrm{~d} \omega=-\frac{1}{2 N} \sum_{j} \int\left(\partial_{j} f\right)\left(\partial_{j} g\right) \mathrm{d} \omega
$$

В явном виде $\widetilde{L}$ задается следующим образом:

$$
\widetilde{L}=L-\sum_{j} b_{j} \partial_{j}, \quad b_{j}=W_{j}^{\prime}\left(x_{j}\right)=\frac{x_{j}-\gamma_{j}}{R^{2}} .
$$

Так как дополнительный потенциал $W_{j}$ является равномерно выпуклым и удовлетворяет неравенству

$$
\inf _{j} \inf _{x \in \mathbb{R}} W_{j}^{\prime \prime}(x) \geqslant R^{-2},
$$

то из (3.6) и $\beta \geqslant 1$ вытекает, что

$$
\left\langle\mathbf{v}, \nabla^{2} \widetilde{\mathscr{H}}(\mathbf{x}) \mathbf{v}\right\rangle \geqslant \frac{1}{R^{2}}\|\mathbf{v}\|^{2}+\frac{1}{N} \sum_{i<j} \frac{\left(v_{i}-v_{j}\right)^{2}}{\left(x_{i}-x_{j}\right)^{2}}, \quad \mathbf{v} \in \mathbb{R}^{N} .
$$

Множитель $R^{-2}$ в первом члене происходит из дополнительной выпуклости локального взаимодействия и улучшает локальное рассеяние формы Дирихле. В частности, имеет место равномерная нижняя оценка

$$
\nabla^{2} \widetilde{\mathscr{H}}=\operatorname{Hess}(-\log \omega) \geqslant R^{-2} .
$$

Это показывает, что время прихода в равновесное состояние $\omega$ для $\widetilde{L}$-динамики ограничено сверху величиной $C R^{2}$.

Первым шагом в доказательстве теоремы 3.3 является анализ локального релаксационного потока, который удовлетворяет логарифмическому неравенству Соболева и следующей оценке рассеяния.

Теорема 3.4. Пусть выполнено неравенство (3.39). Рассмотрим уравнение эволюиии

$$
\partial_{t} q_{t}=\widetilde{L} q_{t}, \quad t \geqslant 0
$$


с начальным условием $q_{0}=q$ и с обратимой мерой $\omega$. Предположим, что $q \in L^{\infty}(\mathrm{d} \omega)$. Тогда мъ имеем оценки

$$
\begin{gathered}
\partial_{t} D_{\omega}\left(\sqrt{q_{t}}\right) \leqslant-\frac{1}{R^{2}} D_{\omega}\left(\sqrt{q_{t}}\right)-\frac{1}{2 N^{2}} \int \sum_{i, j=1}^{N} \frac{\left(\partial_{i} \sqrt{q_{t}}-\partial_{j} \sqrt{q_{t}}\right)^{2}}{\left(x_{i}-x_{j}\right)^{2}} \mathrm{~d} \omega \\
\frac{1}{2 N^{2}} \int_{0}^{\infty} \mathrm{d} s \int \sum_{i, j=1}^{N} \frac{\left(\partial_{i} \sqrt{q_{s}}-\partial_{j} \sqrt{q_{s}}\right)^{2}}{\left(x_{i}-x_{j}\right)^{2}} \mathrm{~d} \omega \leqslant D_{\omega}(\sqrt{q})
\end{gathered}
$$

и логарифмическое неравенство Соболева

$$
S_{\omega}(q) \leqslant C R^{2} D_{\omega}(\sqrt{q})
$$

с универсальной константой C. Следовательно, время прихода в равновесие имеет порядок $R^{2}$ :

$$
S_{\omega}\left(q_{t}\right) \leqslant e^{-C t / R^{2}} S_{\omega}(q) .
$$

ДокАЗАтЕЛьство. Эту теорему можно доказать, используя стандартное рассуждение из п. 3.2.1. Ключевым дополнительным соображением является выпуклая оценка (3.6), которая приводит ко второму члену в правой части неравенства (3.41) из последней строки соотношения (3.19). В частности, это дает верхнюю оценку на $\partial_{t} D_{\omega}\left(\sqrt{q_{t}}\right)$, и, интегрируя (3.41), мы получаем $(3.42)$.

Оценка (3.42) на второй член в неравенстве (3.39) играет ключевую роль в следующей теореме.

ТеОрема 3.5. Предположим, что выполнено условие $I$, и пусть $q \in L^{\infty}-$ некоторая плотность, $\int q \mathrm{~d} \omega=1$. Фиксируем $n \geqslant 1, \mathbf{m} \in \mathscr{N}_{+}^{n}$ u пусть $G: \mathbb{R}^{n} \rightarrow \mathbb{R}$ - ограниченная гладкая функиия с компактным носителем, а величина $\mathscr{G}_{i, \mathbf{m}}$ определена в (3.29). Тогда для любого $J \subset\left\{1,2, \ldots, N-m_{n}\right\}$ и для любого $\tau>0$ мы имеем

$$
\begin{aligned}
& \left|\int \frac{1}{N} \sum_{i \in J} \mathscr{G}_{i, \mathbf{m}}(\mathbf{x}) q \mathrm{~d} \omega-\int \frac{1}{N} \sum_{i \in J} \mathscr{G}_{i, \mathbf{m}}(\mathbf{x}) \mathrm{d} \omega\right| \\
& \leqslant C\left(\frac{|J| D_{\omega}(\sqrt{q}) \tau}{N^{2}}\right)^{1 / 2}+C \sqrt{S_{\omega}(q)} e^{-c \tau / R^{2}} .
\end{aligned}
$$

ДокАЗАТЕЛьСтво. Для простоты рассмотрим случай $\mathbf{m}=(1,2, \ldots, n)$, общий случай будет получаться соответствующим переопределением функции $G$. Пусть $q_{t}$ удовлетворяет уравнению

$$
\partial_{t} q_{t}=\widetilde{L} q_{t}, \quad t \geqslant 0,
$$

с начальным условием $q_{0}=q$. Мы имеем

$$
\int\left[\frac{1}{N} \sum_{i \in J} \mathscr{G}_{i, \mathbf{m}}\right](q-1) \mathrm{d} \omega=\int\left[\frac{1}{N} \sum_{i \in J} \mathscr{G}_{i, \mathbf{m}}\right]\left(q-q_{\tau}\right) \mathrm{d} \omega+\int\left[\frac{1}{N} \sum_{i \in J} \mathscr{G}_{i, \mathbf{m}}\right]\left(q_{\tau}-1\right) \mathrm{d} \omega .
$$


Оценка на второй член может быть получена из неравенства энтропии (3.16), прихода энтропии в равновесие (3.44) и ограниченности функции $G$; это дает второй член в неравенстве (3.45).

Для оценки первого члена в (3.46) произведем следующее вычисление, которое получается дифференцированием с использованием уравнения $\partial q_{t}=\widetilde{L} q_{t}$ и соотношения $(3.36)$ :

$$
\begin{aligned}
\int \frac{1}{N} \sum_{i} \mathscr{G}_{i, \mathbf{m}}(\mathbf{x}) q_{\tau} \mathrm{d} \omega-\int \frac{1}{N} \sum_{i} \mathscr{G}_{i, \mathbf{m}}(\mathbf{x}) q_{0} \mathrm{~d} \omega \\
=\int_{0}^{\tau} \mathrm{d} s \int \frac{1}{N} \sum_{i} \sum_{k=1}^{n} \partial_{k} G\left(N\left(x_{i}-x_{i+1}\right), \ldots, N\left(x_{i+n-1}-x_{i+n}\right)\right) \\
\times\left[\partial_{i+k-1} q_{s}-\partial_{i+k} q_{s}\right] \mathrm{d} \omega .
\end{aligned}
$$

В силу неравенства Коши-Буняковского и соотношения $\partial q=2 \sqrt{q} \partial \sqrt{q}$, последний член оценивается сверху величиной

$$
\begin{aligned}
2 \sum_{k=1}^{n}\left[\int_{0}^{\tau}\right. & \mathrm{d} s \int \sum_{i}\left[\partial_{k} G\left(N\left(x_{i}-x_{i+1}\right), \ldots, N\left(x_{i+n-1}-x_{i+n}\right)\right)\right]^{2} \\
& \left.\times\left(x_{i+k-1}-x_{i+k}\right)^{2} q_{s} \mathrm{~d} \omega\right]^{1 / 2} \\
& \times\left[\int_{0}^{\tau} \mathrm{d} s \int \frac{1}{N^{2}} \sum_{i} \frac{1}{\left(x_{i+k-1}-x_{i+k}\right)^{2}}\left[\partial_{i+k-1} \sqrt{q_{s}}-\partial_{i+k} \sqrt{q_{s}}\right]^{2} \mathrm{~d} \omega\right]^{1 / 2} \\
\leqslant & C\left(\frac{|J| D_{\omega}\left(\sqrt{q_{0}}\right) \tau}{N^{2}}\right)^{1 / 2}
\end{aligned}
$$

где мы использовали неравенство (3.42) и тот факт, что

$$
\begin{gathered}
{\left[\partial_{k} G\left(N\left(x_{i}-x_{i+1}\right), \ldots, N\left(x_{i+k-1}-x_{i+k}\right), \ldots, N\left(x_{i+n-1}-x_{i+n}\right)\right)\right]^{2}} \\
\times\left(x_{i+k-1}-x_{i+k}\right)^{2} \leqslant C N^{-2},
\end{gathered}
$$

так как функция $G$ гладкая и имеет компактный носитель. Теорема доказана.

Для сравнения с теоремой 3.5 мы приведем следующий результат, который доказывается аналогично.

Лемма 3.6. Пусть $G: \mathbb{R} \rightarrow \mathbb{R}$ - ограниченная гладкая функиия с компактным носителем и последовательность $E_{i}$ биксирована. Тогда для любого $\tau>0$ мы имеем

$$
\begin{aligned}
& \left|\frac{1}{N} \sum_{i} \int G\left(N\left(x_{i}-E_{i}\right)\right) q \mathrm{~d} \omega-\frac{1}{N} \sum_{i} \int G\left(N\left(x_{i}-E_{i}\right)\right) \mathrm{d} \omega\right| \\
& \leqslant C \sqrt{S_{\omega}(q) \tau}+C \sqrt{S_{\omega}(q)} e^{-c \tau / R^{2}} .
\end{aligned}
$$

Заметим, что, используя рассеяние локальной формы Дирихле, происходящее из второго члена в правой части неравенства (3.41), мы получили ключевой множитель $N^{-1 / 2}$ в оценке (3.45) по сравнению с (3.48).

Последним шагом в доказательстве теоремы 3.3 являются следующие оценки энтропии и формы Дирихле. 
Теорема 3.7. Предположим, что имеет место оченка (3.6), и напомним, что $\tau=R^{2} N^{\varepsilon^{\prime}} \geqslant 3 t_{0}$, где $t_{0}=N^{-2 \mathfrak{a}}$. Пусть $S_{\mu}\left(f_{t_{0}}\right) \leqslant C N^{m}$ для некоторого фиксированного $\mathrm{m}$. Положим $g_{t}:=f_{t} / \psi$. Тогда имеются следующие оценки на энтропию и форму Дирихле:

$$
S_{\omega}\left(g_{\tau / 2}\right) \leqslant C N R^{-2} Q, \quad D_{\omega}\left(\sqrt{g_{\tau}}\right) \leqslant C N R^{-4} Q .
$$

ДоказАтельство. Напомним, что имеет место уравнение $\partial_{t} f_{t}=L f_{t}$. Стандартная оценка на энтропию функции $f_{t}$ по отношению к инвариантной мере получается дифференцированием энтропии дважды и использованием логарифмического неравенства Соболева (см. п. 3.2.1). Энтропия и форма Дирихле в (3.49), однако, вычисляются по отношению к мере $\omega$. Это дает дополнительный второй член в соотношении (3.18), и мы воспользуемся следующим тождеством [106], которое справедливо для произвольной плотности вероятности $\psi_{t}$ :

$$
\partial_{t} S_{\mu}\left(f_{t} \mid \psi_{t}\right)=-\frac{2}{N} \sum_{j} \int\left(\partial_{j} \sqrt{g_{t}}\right)^{2} \psi_{t} \mathrm{~d} \mu+\int g_{t}\left(L-\partial_{t}\right) \psi_{t} \mathrm{~d} \mu,
$$

где $g_{t}:=f_{t} / \psi_{t}$, a

$$
S_{\mu}\left(f_{t} \mid \psi_{t}\right):=\int f_{t} \log \frac{f_{t}}{\psi_{t}} \mathrm{~d} \mu
$$

есть относительная энтропия.

В наших приложениях мы будем считать $\psi_{t}$ не зависящей от времени, т. е. $\psi_{t}=\psi=\omega / \mu$, откуда $S_{\mu}\left(f_{t} \mid \psi\right)=S_{\omega}\left(g_{t}\right)$. Используя (3.37), мы получаем

$$
\partial_{t} S_{\omega}\left(g_{t}\right)=-\frac{2}{N} \sum_{j} \int\left(\partial_{j} \sqrt{g_{t}}\right)^{2} \mathrm{~d} \omega+\int \widetilde{L} g_{t} \mathrm{~d} \omega+\sum_{j} \int b_{j} \partial_{j} g_{t} \mathrm{~d} \omega .
$$

Так как мера $\omega$ является $\widetilde{L}$-инвариантной, то средний член в правой части обращается в нуль, и мы получаем из неравенства Коши-Буняковского следующее:

$$
\partial_{t} S_{\omega}\left(g_{t}\right) \leqslant-D_{\omega}\left(\sqrt{g_{t}}\right)+C N \sum_{j} \int b_{j}^{2} g_{t} \mathrm{~d} \omega \leqslant-D_{\omega}\left(\sqrt{g_{t}}\right)+C N \Lambda,
$$

где мы определили

$$
\Lambda:=Q R^{-4}=\sup _{t \geqslant 0} R^{-4} \sum_{j} \int\left(x_{j}-\gamma_{j}\right)^{2} f_{t} \mathrm{~d} \mu .
$$

С учетом (3.43), мы имеем

$$
\partial_{t} S_{\omega}\left(g_{t}\right) \leqslant-C R^{-2} S_{\omega}\left(g_{t}\right)+C N \Lambda, \quad t \geqslant N^{-2 \mathfrak{a}} .
$$

Чтобы получить первое неравенство из (3.49), мы проинтегрируем неравенство (3.52) от $t_{0}=N^{-2 \mathfrak{a}}$ до $\tau / 2$, используя тот факт, что $\tau=R^{2} N^{\varepsilon^{\prime}}$ и $S_{\omega}\left(g_{t_{0}}\right) \leqslant$ $C N^{m}+N^{2} Q$ для некоторого конечного $m$, зависящего от $\mathfrak{a}$. Эта априорная оценка вытекает из неравенства

$$
S_{\omega}\left(g_{t_{0}}\right)=S_{\mu}\left(f_{t_{0}} \mid \psi\right)=S_{\mu}\left(f_{t_{0}}\right)-\log Z+\log \widetilde{Z}+N \int f_{t_{0}} W \mathrm{~d} \mu \leqslant C N^{m}+N^{2} Q,
$$


где мы использовали неравенства $|\log Z| \leqslant C N^{m}$ и $|\log \widetilde{Z}| \leqslant C N^{m}$, которые могут быть проверены непосредственно. Второе неравенство в (3.49) можно получить из первого, интегрируя неравенство (3.50) от $t=\tau / 2$ до $t=\tau$ и используя монотонность формы Дирихле по времени.

Теперь, наконец, мы можем завершить доказательство теоремы 3.3. Напомним, что $\tau=R^{2} N^{\varepsilon^{\prime}}$ и $t_{0}=N^{-2 \mathfrak{a}}$. Возьмем $q_{\tau}:=g_{\tau}=f_{\tau} / \psi$ в качестве плотности $q$ в теореме 3.5. Условие $q_{\tau} \in L^{\infty}$ может быть обеспечено рассуждением с приближениями. Тогда из теоремы 3.7, теоремы 3.5 вместе с неравенством (3.53) и соотношения $\Lambda \tau=Q \tau^{-1} N^{2 \varepsilon^{\prime}}$ непосредственно вытекает неравенство

$$
\left|\int \frac{1}{N} \sum_{i \in J} \mathscr{G}_{i, \mathbf{m}} f_{\tau} \mathrm{d} \mu-\int \frac{1}{N} \sum_{i \in J} \mathscr{G}_{i, \mathbf{m}} \mathrm{d} \omega\right| \leqslant C N^{\varepsilon^{\prime}} \sqrt{|J| Q(\tau N)^{-1}}+C e^{-c N^{\varepsilon^{\prime}}},
$$

т. е. локальные статистики для $f_{\tau} \mu$ и $\omega$ могут быть сравнены. Очевидно, что неравенство (3.54) также выполнено при специальном выборе $f_{0}=1$ (для которого $\left.f_{\tau}=1\right)$, т. е. локальные статистики для $\mu$ и $\omega$ также могут быть сравнены. Это завершает доказательство теоремы 3.3 .

\section{3. От распределения промежутков к корреляционным функци-} ям: набросок доказательства теоремы 3.1. Наш основной результат теорема 3.1 - будет вытекать из теоремы 3.3 и того факта, что в случае $\tau \geqslant$ $N^{-2 \mathfrak{a}+\delta}$ условие $(3.9)$ влечет

$$
N^{\varepsilon^{\prime}} \sqrt{|J| Q(\tau N)^{-1}} \leqslant N^{\varepsilon^{\prime}-\delta / 2}=N^{-\delta / 6} \rightarrow 0
$$

при выборе $\varepsilon^{\prime}=\delta / 3$ и $|J| \leqslant N$. Следовательно, локальные статистики наблюдаемых, зависящих от разностей собственных значений, совпадают в пределе при $N \rightarrow \infty$.

Для завершения доказательства теоремы 3.1 нам нужно показать, что сходимости наблюдаемых $\mathscr{G}_{i, \mathbf{m}}$ достаточно для того, чтобы определить корреляционные функции от переменных $x_{i}$ в смысле, описанном в теореме 3.1. Это получается при помощи довольно стандартного технического рассуждения, подробности которого даны в приложении В. Здесь мы лишь обозначим основные моменты.

Теорема 3.3 выявляет совместное поведение разностей собственных значений в правильном масштабе $1 / N$, благодаря множителю $N$ в аргументе функции $\mathscr{G}$ из (3.29). Небольшая техническая трудность заключается в том, что наблюдаемая (3.29) и ее усредненная версия (3.30) включают фиксированные индексы собственных значений, в то время как корреляционные функции включают кумулятивные статистики.

Чтобы понять эту тонкость, рассмотрим для простоты случай $n=1$ и положим, например, $m_{1}=1$. Наблюдаемая (3.30) дает ответ на вопрос каково эмпирическое распределение разностей последовательных собственных значений? Другими словами, (3.30) непосредственно определяет распределение промежутков, введенное в п. 1.5.1. Корреляционные функции дают ответ на вопрос "какова вероятность того, что два собственных значения находятся на данном расстоянии друг от друга?", т. е. корреляционные функции непосредственным образом не чувствительны к наличию возможных собственных 
значений между двумя данными. Конечно, эти два вопроса тесно связаны друг с другом и из ответа на один из них легко вытекает ответ на другой. В одном направлении это как раз было получено в результате вычисления (1.38), и теперь нам необходимо пройти и в обратном направлении, т. е. получить корреляционные функции из (обобщенных) распределений промежутков.

В действительности в этом направлении результат проще, и его суть заключена в следующей формуле:

$$
\begin{gathered}
\int_{E-b}^{E+b} \frac{\mathrm{d} E^{\prime}}{2 b} \int_{\mathbb{R}^{n}} \mathrm{~d} \alpha_{1} \cdots \mathrm{d} \alpha_{n} O\left(\alpha_{1}, \ldots, \alpha_{n}\right) p_{\tau, N}^{(n)}\left(E^{\prime}+\frac{\alpha_{1}}{N \varrho(E)}, \ldots, E^{\prime}+\frac{\alpha_{n}}{N \varrho(E)}\right) \\
=C_{N, n} \int_{E-b}^{E+b} \frac{\mathrm{d} E^{\prime}}{2 b} \int \sum_{i_{1} \neq i_{2} \neq \cdots \neq i_{n}} \widetilde{O}\left(N\left(x_{i_{1}}-E^{\prime}\right), N\left(x_{i_{1}}-x_{i_{2}}\right),\right. \\
\left.\quad \ldots, N\left(x_{i_{n-1}}-x_{i_{n}}\right)\right) f_{\tau} \mathrm{d} \mu \\
=C_{N, n} \int_{E-b}^{E+b} \frac{\mathrm{d} E^{\prime}}{2 b} \int \sum_{\mathbf{m} \in S_{n}} \sum_{i=1}^{N} Y_{i, \mathbf{m}}\left(E^{\prime}, \mathbf{x}\right) f_{\tau} \mathrm{d} \mu,
\end{gathered}
$$

где $C_{N, n}:=N^{n}(N-n) ! / N !=1+O_{n}\left(N^{-1}\right)$, мы обозначили через $S_{n}$ сумму набора возрастающих положительных чисел $\mathbf{m}=\left(m_{2}, m_{3}, \ldots, m_{n}\right) \in \mathbb{N}_{+}^{n-1}$, $m_{2}<m_{3}<\cdots<m_{n}$, и ввели величины

$$
\begin{gathered}
Y_{i, \mathbf{m}}\left(E^{\prime}, \mathbf{x}\right):=\widetilde{O}\left(N\left(x_{i}-E^{\prime}\right), N\left(x_{i}-x_{i+m_{2}}\right), \ldots, N\left(x_{i}-x_{i+m_{n}}\right)\right), \\
\widetilde{O}\left(u_{1}, u_{2}, \ldots, u_{n}\right):=O\left(\varrho(E) u_{1}, \varrho(E)\left(u_{1}-u_{2}\right), \ldots\right) .
\end{gathered}
$$

Мы положим также $Y_{i, \mathbf{m}}=0$ при $i+m_{n}>N$. Первое из равенств (3.55) есть просто определение корреляционной функции после тривиальной перенормировки. Что касается перехода от второй к третьей строке, сначала заметим, что в силу симметрии величины $p_{\tau, N}^{(n)}$ относительно перестановок мы можем предположить, что функция $O$ симметрична, и ограничиться суммированием по $i_{1}<i_{2}<\cdots<i_{n}$ с общим множителем $n$ !. Затем мы сделали замену индексов $i=i_{1}, i_{2}=i+m_{2}, i_{3}=i+m_{3}, \ldots$ и произвели новое суммирование по всем разностям индексов, включенных в $\mathbf{m}$. За исключением первой переменной $N\left(x_{i_{1}}-E^{\prime}\right)$, функция $Y_{i, \mathbf{m}}$ имеет вид $(3.29)$, так что применима теорема 3.3. Зависимость от первой переменной станет несущественной после интегрирования $\mathrm{d} E^{\prime}$ по макроскопическому отрезку.

Для того чтобы иметь контроль над поправочными членами в этом рассуждении, и особенно для того, чтобы доказать, что даже поправочные члены в потенциально бесконечной сумме по всем $\mathbf{m} \in S_{n}$ сходятся, необходимо иметь априорную оценку на локальную плотность. В этом месте используется условие IV (см. (3.10)). Подробности можно найти в приложении В.

\section{4. Теоремы сравнения для функций Грина}

Упрощенный вариант теоремы сравнения для функций Грина уже был сформулирован в качестве теоремы 1.6. Здесь мы приведем полную версию - теорему 4.1. Она быстро приведет нас к теореме 4.2, утверждающей, что корреляционные функции собственных значений двух матричных ансамблей совпадают 
в масштабе $1 / N$ при условии почти полного совпадения первых четырех моментов всех матричных элементов этих двух ансамблей. Здесь мы не предполагаем, что вещественные и комплексные части матричных элементов являются независимыми одинаково распределенными величинами, так что $k$-й момент для $h_{i j}$ понимается как набор чисел $\int \bar{h}^{s} h^{k-s} \nu_{i j}(\mathrm{~d} h), s=0,1, \ldots, k$. Похожая теорема 1.7, доказанная в [17], сравнивает совместные распределения индивидуальных собственных значений, что не покрывается нашей теоремой 4.1, но этот результат не применим непосредственно к матричным элементам функций Грина. В п. 4.3 мы схематически изложим некоторые идеи доказательства теоремы 1.7 для того, чтобы указать на различия между двумя результатами. Основным средством для обеих теорем является локальный полукруговой закон в практически оптимальном масштабе $N^{-1+\varepsilon}$. Возмущение собственных значений, использованное в теореме 1.7, требует некоторых оценок на отталкивание уровней. В то же время доказательство теоремы 4.1 опирается лишь на простую теорию возмущения резольвенты.

Теорема 4.1 (сравнение функций Грина [9; теорема 2.3]). Пусть заданы две обобщенные $(N \times N)$-матриць Вигнера $H^{(v)}$ и $H^{(w)}$ с матричнъми элементами, задаваемыми случайными величинами $N^{-1 / 2} v_{i j}$ u $N^{-1 / 2} w_{i j}$ coответственно, где $v_{i j}$ и $w_{i j}$ удовлетворяют условию равномерного субэкспоненциального убывания

$$
\mathrm{P}\left(\left|v_{i j}\right| \geqslant x\right) \leqslant C \exp \left(-x^{\vartheta}\right), \quad \mathrm{P}\left(\left|w_{i j}\right| \geqslant x\right) \leqslant C \exp \left(-x^{\vartheta}\right)
$$

для некоторых $C, \vartheta>0$. Забиксируем биективное упорядочивающее отображение на множестве индексов независимых матричных элементов:

$$
\phi:\{(i, j): 1 \leqslant i \leqslant j \leqslant N\} \rightarrow\{1, \ldots, \gamma(N)\}, \quad \gamma(N):=\frac{N(N+1)}{2},
$$

и обозначим через $H_{\gamma}$ обобщенную матричу Вигнера, матричные элементы $h_{i j}$ которой удовлетворяют v-распределению при $\phi(i, j) \leqslant \gamma u$ w-распределению в противном случае. $B$ частности, $H^{(v)}=H_{0} u H^{(w)}=H_{\gamma(N)}$. Рассмотрим произвольное $\kappa>0$ и предположим, что для любого малого параметра $\tau>0$ и для любого $y \geqslant N^{-1+\tau}$ мы имеем следуюшую оценку на диагональные элементы резольвенты:

$$
\mathrm{P}\left(\max _{0 \leqslant \gamma \leqslant \gamma(N)} \max _{1 \leqslant k \leqslant N} \max _{|E| \leqslant 2-\kappa}\left|\left(\frac{1}{H_{\gamma}-E-i y}\right)_{k k}\right| \leqslant N^{2 \tau}\right) \geqslant 1-C N^{-c \log \log N}
$$

с некоторыми константами $C$, с, зависящими только от $\tau, \kappa$. Более того, предположим, что первые три момента для $v_{i j} u w_{i j}$ совпадают, т.е.

$$
\mathrm{E} \bar{v}_{i j}^{s} v_{i j}^{u}=\mathrm{E} \bar{w}_{i j}^{s} w_{i j}^{u}, \quad 0 \leqslant s+u \leqslant 3
$$

а разность между четвертыми моментами для $v_{i j}$ и $w_{i j}$ существенно меньше 1, скажем,

$$
\left|\mathrm{E} \bar{v}_{i j}^{s} v_{i j}^{4-s}-\mathrm{E} \bar{w}_{i j}^{s} w_{i j}^{4-s}\right| \leqslant N^{-\delta}, \quad s=0,1,2,3,4,
$$


для некоторого $\delta>0$. Для произвольного $\varepsilon>0$ выберем $\eta$ так, ито $N^{-1-\varepsilon} \leqslant$ $\eta \leqslant N^{-1}$. Для любой последовательности положительных иелых чисел $k_{1}, \ldots, k_{n}$ рассмотрим комплексные параметры $z_{j}^{m}=E_{j}^{m} \pm i \eta, j=1, \ldots, k_{m}$, $m=1, \ldots, n, c\left|E_{j}^{m}\right| \leqslant 2-2 \kappa$ и с любъм выбором знаков \pm . Пусть $G^{(v)}(z)=$ $\left(H^{(v)}-z\right)^{-1}$ обозначает резольвенту и выберем функиию $F\left(x_{1}, \ldots, x_{n}\right)$ такую, что для любого мультииндекса $\alpha=\left(\alpha_{1}, \ldots, \alpha_{n}\right)$ c $1 \leqslant|\alpha| \leqslant 5$ и для любого достаточно малого $\varepsilon^{\prime}>0$ выполнены неравенства

$$
\max \left\{\left|\partial^{\alpha} F\left(x_{1}, \ldots, x_{n}\right)\right|: \max _{j}\left|x_{j}\right| \leqslant N^{\varepsilon^{\prime}}\right\} \leqslant N^{C_{0} \varepsilon^{\prime}}
$$

$u$

$$
\max \left\{\left|\partial^{\alpha} F\left(x_{1}, \ldots, x_{n}\right)\right|: \max _{j}\left|x_{j}\right| \leqslant N^{2}\right\} \leqslant N^{C_{0}}
$$

для некоторой константы $C_{0}$.

Тогда существует константа $C_{1}$, зависящая от $\vartheta, \sum_{m} k_{m}$ и $C_{0}$, такая, что для любого $\eta$ такого, что $N^{-1-\varepsilon} \leqslant \eta \leqslant N^{-1}$, и любого выбора знаков мнимых частей параметров $z_{j}^{m}$ мь имеем

$$
\begin{aligned}
& \left|\mathrm{E} F\left(\frac{1}{N} \operatorname{Tr}\left[\prod_{j=1}^{k_{1}} G^{(v)}\left(z_{j}^{1}\right)\right], \ldots, \frac{1}{N} \operatorname{Tr}\left[\prod_{j=1}^{k_{n}} G^{(v)}\left(z_{j}^{n}\right)\right]\right)-\mathrm{E} F\left(G^{(v)} \rightarrow G^{(w)}\right)\right| \\
& \leqslant C_{1} N^{-1 / 2+C_{1} \varepsilon}+C_{1} N^{-\delta+C_{1} \varepsilon}
\end{aligned}
$$

где во втором члене в аргументе функции $F$ функции Грина матриць $H^{(v)}$ заменяются на функиии Грина матрицы $H^{(w)}$, а остальные параметры остаются неизменными.

ЗАмЕчАниЕ 3. Мы сформулировали теорему 4.1 для функций от следов мономов функций Грина, так как именно в этом виде она требуется нам для приложений. Однако результат (как и доказательство, которое мы изложим ниже) справедлив также и непосредственно для матричных элементов мономов функций Грина; соответствующее точное утверждение можно найти в [9]. Кроме того, заметим, что теорема 4.1 справедлива для обобщенных матриц Вигнера при условии $C_{\text {sup }}=\sup _{i, j} N \sigma_{i, j}^{2}<\infty$. Наличие положительной нижней оценки на дисперсии, т. е. условие $C_{\mathrm{inf}}>0$ в $(1.17)$, не является необходимым в этой теореме.

ЗАмЕчАниЕ 4. Хотя мы сформулировали теорему 4.1 для эрмитовых и симметрических ансамблей, аналогичные результаты имеют место и для вещественных и комплексных выборочных ковариационных ансамблей, с очевидными изменениями в доказательстве.

Следующий результат является главным следствием теоремы 4.1 и будет доказан далее в этом разделе. Основным утверждением является теорема 4.1, доказательство ее следствия технически довольно несложно.

ТЕОРемА 4.2 (сравнение корреляционных функций [9; теорема 6.4]). Предположим, что выполнены предположения теоремы 4.1. Пусть $p_{v, N}^{(k)} u p_{w, N}^{(k)}-$ 
$k$-точечные функиии собственных значений по отношению $\kappa$ вероятностным законам, задаваемым матрицами $H^{(v)}$ и $H^{(w)}$ соответственно. Тогда для любого $|E|<2$, любого $k \geqslant 1$ и любой тестовой функиии $O: \mathbb{R}^{k} \rightarrow \mathbb{R}$ с компактным носителем мы имеем

$$
\int_{\mathbb{R}^{k}} \mathrm{~d} \alpha_{1} \cdots \mathrm{d} \alpha_{k} O\left(\alpha_{1}, \ldots, \alpha_{k}\right)\left(p_{v, N}^{(k)}-p_{w, N}^{(k)}\right)\left(E+\frac{\alpha_{1}}{N}, \ldots, E+\frac{\alpha_{k}}{N}\right)=0 .
$$

\section{1. Доказательство теоремы 4.1 сравнения для функций Грина.} Основная идея заключается в том, чтобы оценивать результат изменения матричных элементов резольвенты элемент за элементом, при помощи разложения резольвенты. Так как каждый матричный элемент имеет типичный размер $N^{-1 / 2}$, а неравенство (4.1) фактически дает оценку на резольвенты, то разложение резольвенты до четвертого порядка позволит измерить изменение каждого элемента с точностью $O\left(N^{-5 / 2}\right)$ (по модулю некоторых малых поправок порядка $\left.N^{O(\tau)}\right)$. Математические ожидания членов до четвертого порядка включительно зависят только от четырех первых моментов распределения отдельных элементов, которые можно сравнить непосредственно. Поправочные члены являются незначительными, даже если мы суммируем их до $N^{2}$ раз, что соответствует числу шагов сравнения, необходимых для замены всех матричных элементов.

Чтобы начать подробное доказательство, нам вначале потребуется оценка типа (4.1) на резольвенты промежуточных матриц. Из тривиальной оценки

$$
\operatorname{Im}\left(\frac{1}{H-E-i \eta}\right)_{j j} \leqslant\left(\frac{y}{\eta}\right) \operatorname{Im}\left(\frac{1}{H-E-i y}\right)_{j j}, \quad \eta \leqslant y,
$$

и из неравенства (4.1) мы получаем следующую априорную оценку:

$$
\begin{aligned}
& \mathrm{P}\left(\max _{0 \leqslant \gamma \leqslant \gamma(N)} \max _{1 \leqslant k \leqslant N} \max _{|E| \leqslant 2-\kappa} \sup _{\eta \geqslant N^{-1-\varepsilon}}\left|\operatorname{Im}\left(\frac{1}{H_{\gamma}-E \pm i \eta}\right)_{k k}\right| \leqslant N^{3 \tau+\varepsilon}\right) \\
& \geqslant 1-C N^{-c \log \log N} .
\end{aligned}
$$

Заметим, что супремум по $\eta$ может быть включен в данную оценку путем установления вначале оценки для мелкой решетки по $\eta$ с расстоянием $N^{-10}$ с последующим распространением оценки на все $\eta$, используя тот факт, что функции Грина являются липшицево непрерывными по $\eta$ с константой Липшица $\eta^{-2}$.

Пусть $\lambda_{m}$ и $u_{m}$ обозначают собственные значения и собственные векторы матрицы $H_{\gamma}$. Тогда по определению функции Грина мы имеем

$$
\left|\left(\frac{1}{H_{\gamma}-z}\right)_{j k}\right| \leqslant \sum_{m=1}^{N} \frac{\left|u_{m}(j)\right|\left|u_{m}(k)\right|}{\left|\lambda_{m}-z\right|} \leqslant\left[\sum_{m=1}^{N} \frac{\left|u_{m}(j)\right|^{2}}{\left|\lambda_{m}-z\right|}\right]^{1 / 2}\left[\sum_{m=1}^{N} \frac{\left|u_{m}(k)\right|^{2}}{\left|\lambda_{m}-z\right|}\right]^{1 / 2} .
$$

Определим диадическое разложение

$$
\begin{gathered}
U_{n}=\left\{m: 2^{n-1} \eta \leqslant\left|\lambda_{m}-E\right|<2^{n} \eta\right\}, \quad n=1,2, \ldots, n_{0}:=C \log N, \\
U_{0}=\left\{m:\left|\lambda_{m}-E\right|<\eta\right\}, \quad U_{\infty}:=\left\{m: 2^{n_{0}} \eta \leqslant\left|\lambda_{m}-E\right|\right\}
\end{gathered}
$$


и разобьем суммирование по $m$ на $\bigcup_{n} U_{n}$ :

$$
\begin{aligned}
\sum_{m=1}^{N} \frac{\left|u_{m}(j)\right|^{2}}{\left|\lambda_{m}-z\right|} & =\sum_{n} \sum_{m \in U_{n}} \frac{\left|u_{m}(j)\right|^{2}}{\left|\lambda_{m}-z\right|} \leqslant C \sum_{n} \sum_{m \in U_{n}} \operatorname{Im} \frac{\left|u_{m}(j)\right|^{2}}{\lambda_{m}-E-i \cdot 2^{n} \eta} \\
& \leqslant C \sum_{n} \operatorname{Im}\left(\frac{1}{H_{\gamma}-E-i \cdot 2^{n} \eta}\right)_{j j} .
\end{aligned}
$$

Используя оценку (4.1) при $n=0,1, \ldots, n_{0}$ и тривиальную оценку величиной $O(1)$ при $n=\infty$, мы получаем

$$
\begin{aligned}
& \mathrm{P}\left(\sup _{0 \leqslant \gamma \leqslant \gamma(N)} \sup _{1 \leqslant k, \ell \leqslant N} \max _{|E| \leqslant 2-\kappa} \sup _{\eta \geqslant N^{-1-\varepsilon}}\left|\left(\frac{1}{H_{\gamma}-E \pm i \eta}\right)_{k \ell}\right| \leqslant N^{4 \tau+\varepsilon}\right) \\
& \geqslant 1-C N^{-c \log \log N} .
\end{aligned}
$$

Теперь начнем заменять элементы по одному. Для простоты обозначений мы рассмотрим случай, когда тестовая функция $F$ зависит лишь от $n=1$ переменной и $k_{1}=1$, т. е. рассмотрим след монома порядка один; общий случай будет получаться аналогично. Рассмотрим телескопическую сумму разностей математических ожиданий:

$$
\begin{aligned}
\mathrm{E} F( & \left.\frac{1}{N} \operatorname{Tr} \frac{1}{H^{(v)}-z}\right)-\mathrm{E} F\left(\frac{1}{N} \operatorname{Tr} \frac{1}{H^{(w)}-z}\right) \\
& =\sum_{\gamma=1}^{\gamma(N)}\left[\mathrm{E} F\left(\frac{1}{N} \operatorname{Tr} \frac{1}{H_{\gamma}-z}\right)-\mathrm{E} F\left(\frac{1}{N} \operatorname{Tr} \frac{1}{H_{\gamma-1}-z}\right)\right] .
\end{aligned}
$$

Пусть $E^{(i j)}$ обозначает матрицу, имеющую единственный ненулевой элемент на месте $(i, j)$, причем этот элемент равен 1 , т. е. $E_{k \ell}^{(i j)}=\delta_{i k} \delta_{j \ell}$. Фиксируем $\gamma \geqslant 1$, и пусть пара $(i, j)$ определяется условием $\phi(i, j)=\gamma$. Мы сравним $H_{\gamma-1}$ с $H_{\gamma}$. Заметим, что эти две матрицы отличаются лишь элементами на местах $(i, j)$ и $(j, i)$ и могут быть записаны следующим образом:

$$
\begin{aligned}
H_{\gamma-1} & =Q+\frac{1}{\sqrt{N}} V, & V & :=v_{i j} E^{(i j)}+v_{j i} E^{(j i)}, \\
H_{\gamma} & =Q+\frac{1}{\sqrt{N}} W, & W & :=w_{i j} E^{(i j)}+w_{j i} E^{(j i)},
\end{aligned}
$$

где матрица $Q$ имеет нулевые элементы на местах $(i, j)$ и $(j, i)$ и мы положили $v_{j i}:=\bar{v}_{i j}$ при $i<j$ и аналогично для $w$. Определим функции Грина формулами

$$
R:=\frac{1}{Q-z}, \quad S:=\frac{1}{H_{\gamma}-z} .
$$

Вначале докажем, что оценка (4.9) выполнена также и для функции Грина $R$. Для этого рассмотрим следующее матричное равенство, получаемое из разложения резольвенты:

$$
R=S+N^{-1 / 2} S V S+\cdots+N^{-9 / 5}(S V)^{9} S+N^{-5}(S V)^{10} R .
$$


Так как $V$ имеет не более двух ненулевых элементов, то при вычислении $(k, \ell)$-го матричного элемента из этого матричного равенства каждый член будет конечной суммой, включающей элементы матриц $S$ или $R$ и $v_{i j}$, например,

$$
(S V S)_{k \ell}=S_{k i} v_{i j} S_{j \ell}+S_{k j} v_{j i} S_{i \ell}
$$

Используя оценку (4.9) для элементов матрицы $S$, субэкспоненциальное убывание для элемента $v_{i j}$ и тривиальную оценку $\left|R_{i j}\right| \leqslant \eta^{-1}$, мы получаем, что для $R$ справедлива оценка (4.9).

Теперь мы можем приступить к доказательству основного результата путем сравнения резольвент для $H^{(\gamma-1)}$ и $H^{(\gamma)}$ с резольвентой $R$ проверочной матрицы $Q$. Используя разложение резольвенты

$S=R-N^{-1 / 2} R V R+N^{-1}(R V)^{2} R-N^{-3 / 2}(R V)^{3} R+N^{-2}(R V)^{4} R-N^{-5 / 2}(R V)^{5} S$,

мы можем записать

$$
\frac{1}{N} \operatorname{Tr} S=\widehat{R}+\xi, \quad \xi:=\sum_{m=1}^{4} N^{-m / 2} \widehat{R}^{(m)}+N^{-5 / 2} \Omega,
$$

где

$$
\widehat{R}:=\frac{1}{N} \operatorname{Tr} R, \quad \widehat{R}^{(m)}:=(-1)^{m} \frac{1}{N} \operatorname{Tr}(R V)^{m} R, \quad \Omega:=-\frac{1}{N} \operatorname{Tr}(R V)^{5} S .
$$

При вычислении этих следов вклад каждого диагонального элемента в $\widehat{R}, \widehat{R}^{(m)}$ и $\Omega$ является суммой нескольких членов. Например,

$$
\begin{aligned}
\widehat{R}^{(2)}=\frac{1}{N} \sum_{k}[ & R_{k i} v_{i j} R_{j j} v_{j i} R_{i k}+R_{k i} v_{i j} R_{j i} v_{i j} R_{j k} \\
& \left.+R_{k j} v_{j i} R_{i i} v_{i j} R_{j k}+R_{k j} v_{j i} R_{i j} v_{j i} R_{i k}\right],
\end{aligned}
$$

и аналогичные формулы имеют место для других членов. Тогда

$$
\begin{aligned}
\mathrm{E} F\left(\frac{1}{N} \operatorname{Tr} \frac{1}{H_{\gamma}-z}\right) & =\mathrm{E} F(\widehat{R}+\xi) \\
& =\mathrm{E}\left[F(\widehat{R})+F^{\prime}(\widehat{R}) \xi+F^{\prime \prime}(\widehat{R}) \xi^{2}+\cdots+F^{(5)}\left(\widehat{R}+\xi^{\prime}\right) \xi^{5}\right] \\
& =\sum_{m=0}^{5} N^{-m / 2} \mathrm{E} A^{(m)}
\end{aligned}
$$

где $\xi^{\prime}$ есть некоторое число между 0 и $\xi$, зависящее от $\widehat{R}$ и $\xi$, а величины $A^{(m)}$ определяются следующим образом:

$$
A^{(0)}=F(\widehat{R}), \quad A^{(1)}=F^{\prime}(\widehat{R}) \widehat{R}^{(1)}, \quad A^{(2)}=F^{\prime \prime}(\widehat{R})\left(\widehat{R}^{(1)}\right)^{2}+F^{\prime}(\widehat{R}) \widehat{R}^{(2)},
$$

и аналогично для $A^{(3)}$ и $A^{(4)}$. Наконец,

$$
A^{(5)}=F^{\prime}(\widehat{R}) \Omega+F^{(5)}\left(\widehat{R}+\xi^{\prime}\right)\left(\widehat{R}^{(1)}\right)^{5}+\cdots .
$$


Математические ожидания величин $A^{(m)}, m \leqslant 4$, по отношению к $v_{i j}$ определяются первыми четырьмя моментами распределения $v_{i j}$, например,

$$
\begin{aligned}
\mathrm{E} A^{(2)}=F^{\prime} & (\widehat{R})\left[\frac{1}{N} \sum_{k} R_{k i} R_{j j} R_{i k}+\cdots\right] \mathrm{E}\left|v_{i j}\right|^{2} \\
& +F^{\prime \prime}(\widehat{R})\left[\frac{1}{N^{2}} \sum_{k, \ell} R_{k i} R_{j \ell} R_{\ell j} R_{i k}+\cdots\right] \mathrm{E}\left|v_{i j}\right|^{2}, \\
& +F^{\prime}(\widehat{R})\left[\frac{1}{N} \sum_{k} R_{k i} R_{j i} R_{j k}+\cdots\right] \mathrm{E} v_{i j}^{2} \\
& +F^{\prime \prime}(\widehat{R})\left[\frac{1}{N^{2}} \sum_{k, \ell} R_{k i} R_{j \ell} R_{\ell i} R_{j k}+\cdots\right] \mathrm{E} v_{i j}^{2} .
\end{aligned}
$$

Заметим, что коэффициенты зависят от четырех производных функции $F$ и от нормированных сумм элементов матрицы $R$. Используя оценку $(4.9)$ для $R$ и оценки на производные (4.3) для типичных значений $\widehat{R}$, мы получаем, что все эти коэффициенты ограничиваются сверху величиной $N^{C(\tau+\varepsilon)}$ с очень большой вероятностью, где $C$ - явно задаваемая константа. Мы также используем оценку (4.4) для экстремальных значений $\widehat{R}$, но это событие имеет очень малую вероятность в силу (4.9). Следовательно, коэффициенты моментов $\mathrm{E} \bar{v}_{i j}^{s} v_{i j}^{u}$, $u+s \leqslant 4$, в величинах $A^{(0)}, \ldots, A^{(4)}$ допускают оценки по модулю множителей $N^{C(\tau+\varepsilon)}$. Заметим, что четвертый момент для $v_{i j}$ появляется только в члене с $m=4$ и уже содержит множитель $N^{-2}$ в (4.11). Поэтому для того, чтобы вычислить $m \leqslant 4$ членов в разложении (4.11) с точностью $o\left(N^{-2}\right)$, достаточно знать первые три момента для $v_{i j}$ точно и четвертый момент лишь с точностью $N^{-\delta}$. Если же $\tau$ и $\varepsilon$ выбраны таким образом, что $C(\tau+\varepsilon)<\delta$, то разница в четвертых моментах не имеет значения.

Наконец, нам нужно оценить поправочный член $A^{(5)}$. Все члены, не содержащие $\Omega$, могут быть рассмотрены как выше: после получения оценок на производные функции $F$ величиной $N^{C(\tau+\varepsilon)}$ можно перейти к математическому ожиданию по отношению к $v_{i j}$, которое не зависит от $\widehat{R}^{(m)}$. Для членов, содержащих $\Omega$, можно рассуждать аналогично, используя тот факт, что элементы матрицы $S$ также, в сущности, ограничены сверху величиной $N^{C(\tau+\varepsilon)}$ (см. (4.9)) и что $v_{i j}$ имеет субэкспоненциальное убывание. С другой стороны, можно использовать неравенство Гёльдера для отделения $S$ от остальных членов, а затем непосредственно воспользоваться оценкой (4.9), например,

$$
\begin{aligned}
\mathrm{E}\left|F^{\prime}(\widehat{R}) \Omega\right| & =\frac{1}{N} \mathrm{E}\left|F^{\prime}(\widehat{R}) \operatorname{Tr}(R V)^{5} S\right| \\
& \leqslant \frac{1}{N}\left[\mathrm{E}\left(F^{\prime}(\widehat{R})\right)^{2} \operatorname{Tr} S^{2}\right]^{1 / 2}\left[\mathrm{E} \operatorname{Tr}(R V)^{5}\left(V R^{*}\right)^{5}\right]^{1 / 2} \leqslant C N^{-5 / 2+C(\tau+\varepsilon)} .
\end{aligned}
$$

Заметим, что в точности то же самое разложение возмущений имеет место для резольвенты матрицы $H_{\gamma-1}$, необходимо лишь везде заменить $v_{i j}$ на $w_{i j}$. В силу условия совпадения моментов, значения математических ожиданий $\mathrm{E} A^{(m)}$ членов с $m \leqslant 3$ в (4.11) совпадают, а для членов с $m=4$ отличаются 
на $N^{-\delta+C(\tau+\varepsilon)}$. Положив $\tau=\varepsilon$, мы получаем

$$
\mathrm{E} F\left(\frac{1}{N} \operatorname{Tr} \frac{1}{H_{\gamma}-z}\right)-\mathrm{E} F\left(\frac{1}{N} \operatorname{Tr} \frac{1}{H_{\gamma-1}-z}\right) \leqslant C N^{-5 / 2+C \varepsilon}+C N^{-2-\delta+C \varepsilon} .
$$

Производя суммирование в (4.10), мы доказываем, что

$$
\mathrm{E} F\left(\frac{1}{N} \operatorname{Tr} \frac{1}{H^{(v)}-z}\right)-\mathrm{E} F\left(\frac{1}{N} \operatorname{Tr} \frac{1}{H^{(w)}-z}\right) \leqslant C N^{-1 / 2+C \varepsilon}+C N^{-\delta+C \varepsilon} .
$$

Это доказательство легко обобщается на функции нескольких переменных. Тем самым доказательство теоремы 4.1 завершено.

\section{2. Доказательство теоремы 4.2 о сравнении корреляционных} функций. Определим приближенную дельта-функцию (с множителем $\pi$ ) в масштабе $\eta$ по формуле

$$
\theta_{\eta}(x):=\operatorname{Im} \frac{1}{x-i \eta}
$$

Мы выберем $\eta \sim N^{-1-\varepsilon}$, т. е. несколько меньше, чем типичное расстояние между собственными значениями. Это означает, что наблюдаемая вида $\theta_{\eta}$ имеет достаточное разрешение, чтобы выявлять индивидуальные собственные значения. Более того, многочлены от таких наблюдаемых выявляют корреляционные функции. С другой стороны,

$$
\frac{1}{N} \operatorname{Im} \operatorname{Tr} G(E+i \eta)=\frac{1}{N} \sum_{i} \theta_{\eta}\left(\lambda_{i}-E\right)
$$

т. е. математические ожидания таких наблюдаемых покрываются неравенством (4.5) из теоремы 4.1. Оставшаяся часть доказательства заключается в придании точного смысла этим соображениям. Необходимо преодолеть две технические трудности. Во-первых, корреляционные функции зависят от различных собственных значений (см. (4.12) ниже), в то время как многочлены резольвенты включают повторный подсчет совпадающих собственных значений. Поэтому необходима формула включения-исключения. Во-вторых, хотя значение $\eta$ намного меньше рассматриваемого масштаба $1 / N$, оно все еще не дает поточечной информации о корреляционных функциях. Однако корреляционные функции в (1.35) определяются только в слабом пределе, т. е. сверяются с некоторой непрерывной функцией $O$. Непрерывность функции $O$ может быть использована для доказательства того, что разность между точными корреляционными функциями и их приближенными значениями в масштабе $\eta \sim N^{-1-\varepsilon}$ незначительна. Этот последний шаг потребует априорной верхней оценки на плотность, которая будет гарантировать, что не очень много собственных значений попадает в пренебрежимо малый отрезок. Такая оценка дана в (4.1), и она в итоге будет проверена при помощи локального полукругового закона.

Для простоты обозначений подробное доказательство будет дано лишь в случае трехточечных корреляционных функций; в общем случае доказательство 
аналогично. По определению корреляционных функций, для любых фиксированных $E, \alpha_{1}, \alpha_{2}, \alpha_{3}$ имеем

$$
\begin{aligned}
\mathrm{E}^{\mathbf{w}} & \frac{1}{N(N-1)(N-2)} \sum_{i \neq j \neq k} \theta_{\eta}\left(\lambda_{i}-E-\frac{\alpha_{1}}{N}\right) \theta_{\eta}\left(\lambda_{j}-E-\frac{\alpha_{2}}{N}\right) \theta_{\eta}\left(\lambda_{k}-E-\frac{\alpha_{3}}{N}\right) \\
& =\int \mathrm{d} x_{1} \mathrm{~d} x_{2} \mathrm{~d} x_{3} p_{w, N}^{(3)}\left(x_{1}, x_{2}, x_{3}\right) \theta_{\eta}\left(x_{1}-E_{1}\right) \theta_{\eta}\left(x_{2}-E_{2}\right) \theta_{\eta}\left(x_{3}-E_{3}\right),
\end{aligned}
$$

где $E_{j}:=E+\alpha_{j} / N$ и $\mathrm{E}^{\mathbf{w}}$ обозначает математическое ожидание по отношению к величинам w. В силу принципа включения-исключения,

$$
\mathrm{E}^{\mathrm{w}} \frac{1}{N(N-1)(N-2)} \sum_{i \neq j \neq k} \theta_{\eta}\left(x_{1}-E_{1}\right) \theta_{\eta}\left(x_{2}-E_{2}\right) \theta_{\eta}\left(x_{3}-E_{3}\right)=\mathrm{E}^{\mathbf{w}} A_{1}+\mathrm{E}^{\mathbf{w}} A_{2}+\mathrm{E}^{\mathbf{w}} A_{3},
$$

где

$$
\begin{aligned}
& A_{1}:=\frac{1}{N(N-1)(N-2)} \prod_{j=1}^{3}\left[\frac{1}{N} \sum_{i} \theta_{\eta}\left(\lambda_{i}-E_{j}\right)\right] \\
& A_{3}:=\frac{2}{N(N-1)(N-2)} \sum_{i} \theta_{\eta}\left(\lambda_{i}-E_{1}\right) \theta_{\eta}\left(\lambda_{i}-E_{2}\right) \theta_{\eta}\left(\lambda_{i}-E_{3}\right)+\cdots
\end{aligned}
$$

и

$$
A_{2}:=B_{1}+B_{2}+B_{3}
$$

где

$$
B_{3}=-\frac{1}{N(N-1)(N-2)} \sum_{i} \theta_{\eta}\left(\lambda_{i}-E_{1}\right) \theta_{\eta}\left(\lambda_{i}-E_{2}\right) \sum_{k} \theta_{\eta}\left(\lambda_{k}-E_{3}\right),
$$

$B_{1}$, аналогичным образом, состоит из членов с $j=k$, а $B_{2}$ состоит из членов c $i=k$.

Заметим, что, по модулю тривиальной замены множителя, значение $\mathrm{E}^{\mathbf{w}} A_{1}$ может быть приближено величиной

$$
\mathrm{E}^{\mathbf{w}} F\left(\frac{1}{N} \operatorname{Im} \operatorname{Tr} \frac{1}{H^{(v)}-z_{1}}, \frac{1}{N} \operatorname{Im} \operatorname{Tr} \frac{1}{H^{(v)}-z_{2}}, \frac{1}{N} \operatorname{Im} \operatorname{Tr} \frac{1}{H^{(v)}-z_{3}}\right),
$$

где функцию $F$ выбираем следующим образом: $F\left(x_{1}, x_{2}, x_{3}\right):=x_{1} x_{2} x_{3}$ при $\max _{j}\left|x_{j}\right| \leqslant N^{\varepsilon}$, и затем ее гладко срезаем, устремляя к нулю в режиме $\max _{j}\left|x_{j}\right| \geqslant N^{2 \varepsilon}$. Разность между математическими ожиданиями величин $F$ и $A_{1}$ пренебрежимо мала, так как она происходит из режима $N^{\varepsilon} \leqslant \max _{j} \frac{1}{N} \times$ $\left|\operatorname{Im} \operatorname{Tr}\left(H^{(v)}-z_{j}\right)^{-1}\right| \leqslant N^{2}$, который имеет экспоненциально малую вероятность в силу (4.9) (верхняя оценка на функцию Грина всегда выполнена, так как $\left.\eta \geqslant N^{-2}\right)$. Здесь аргументами функции $F$ являются мнимые части следов функций Грина, но к этому типу функций можно применять теорему 4.1, так как

$$
\operatorname{Im} \operatorname{Tr} G(z)=\frac{1}{2}[\operatorname{Tr} G(z)-\operatorname{Tr} G(\bar{z})]
$$


Заметим, что главное условие (4.1) в теореме 4.1 выполнено, что вытекает из одной из теорем о локальном полукруговом законе (например, из теоремы 2.5 при $M \sim N$ или из теоремы 2.19).

Аналогично, мы можем приблизить $\mathrm{E}^{\mathrm{w}} B_{3}$ величиной

$$
\mathrm{E}^{\mathbf{w}} G\left(\frac{1}{N^{2}} \operatorname{Tr}\left\{\operatorname{Im} \frac{1}{H^{(v)}-z_{1}} \operatorname{Im} \frac{1}{H^{(v)}-z_{2}}\right\}, \frac{1}{N} \operatorname{Im} \operatorname{Tr} \frac{1}{H^{(v)}-z_{3}}\right),
$$

где $G\left(x_{1}, x_{2}\right)=x_{1} x_{2}$ с соответствующей срезкой для больших значений аргументов. Имеются аналогичные выражения для $B_{1}, B_{2}$ и также для $A_{3}$, в последнем случае выражение включает след произведения трех резольвент. По теореме 4.1, эти математические ожидания по отношению $\mathrm{k} \mathbf{w}$ в приближениях для $\mathrm{E}^{\mathrm{w}} A_{i}$ могут быть заменены на математические ожидания по отношению к v c пренебрежимо малыми ошибками, при условии $\eta \geqslant N^{-1-\varepsilon}$. Итак, мы доказали, что

$$
\begin{gathered}
\lim _{N \rightarrow \infty} \int \mathrm{d} x_{1} \mathrm{~d} x_{2} \mathrm{~d} x_{3}\left[p_{w, N}^{(3)}\left(x_{1}, x_{2}, x_{3}\right)-p_{v, N}^{(3)}\left(x_{1}, x_{2}, x_{3}\right)\right] \\
\times \theta_{\eta}\left(x_{1}-E_{1}\right) \theta_{\eta}\left(x_{2}-E_{2}\right) \theta_{\eta}\left(x_{3}-E_{3}\right)=0 .
\end{gathered}
$$

Положим $\eta=N^{-1-\varepsilon}$ до конца доказательства. Теперь покажем, что выполнение соотношения (4.14) для любых значений $E, \alpha_{1}, \alpha_{2}, \alpha_{3}$ (напомним, что $\left.E_{j}=E+\alpha_{j} / N\right)$ влечет, что перенормированные корреляционные функции $p_{w, N}^{(3)}\left(E+\beta_{1} / N, E+\beta_{2} / N, E+\beta_{3} / N\right)$ и $p_{v, N}^{(3)}\left(E+\beta_{1} / N, E+\beta_{2} / N, E+\beta_{3} / N\right)$ имеют совпадающие слабые пределы как функции от переменных $\beta_{1}, \beta_{2}, \beta_{3}$.

Пусть $O$ - гладкая тестовая функция с компактным носителем, и пусть

$$
\begin{aligned}
O_{\eta}\left(\beta_{1}, \beta_{2}, \beta_{3}\right):=\frac{1}{(\pi N)^{3}} \int_{\mathbb{R}^{3}} \mathrm{~d} \alpha_{1} \mathrm{~d} \alpha_{2} \mathrm{~d} \alpha_{3} O\left(\alpha_{1}, \alpha_{2}, \alpha_{3}\right) \\
\quad \times \theta_{\eta}\left(\frac{\beta_{1}-\alpha_{1}}{N}\right) \theta_{\eta}\left(\frac{\beta_{2}-\alpha_{2}}{N}\right) \theta_{\eta}\left(\frac{\beta_{3}-\alpha_{3}}{N}\right)
\end{aligned}
$$

- ее сглаживание в масштабе $N \eta$. Тогда мы можем записать

$$
\begin{aligned}
\int_{\mathbb{R}^{3}} \mathrm{~d} \beta_{1} \mathrm{~d} \beta_{2} \mathrm{~d} \beta_{3} O\left(\beta_{1}, \beta_{2}, \beta_{3}\right) p_{w, N}^{(3)}\left(E+\frac{\beta_{1}}{N}, E+\frac{\beta_{2}}{N}, E+\frac{\beta_{3}}{N}\right) \\
=\int_{\mathbb{R}^{3}} \mathrm{~d} \beta_{1} \mathrm{~d} \beta_{2} \mathrm{~d} \beta_{3} O_{\eta}\left(\beta_{1}, \beta_{2}, \beta_{3}\right) p_{w, N}^{(3)}\left(E+\frac{\beta_{1}}{N}, E+\frac{\beta_{2}}{N}, E+\frac{\beta_{3}}{N}\right) \\
\quad+\int_{\mathbb{R}^{3}} \mathrm{~d} \beta_{1} \mathrm{~d} \beta_{2} \mathrm{~d} \beta_{3}\left(O-O_{\eta}\right)\left(\beta_{1}, \beta_{2}, \beta_{3}\right) p_{w, N}^{(3)}\left(E+\frac{\beta_{1}}{N}, E+\frac{\beta_{2}}{N}, E+\frac{\beta_{3}}{N}\right) .
\end{aligned}
$$

Первый член в правой части после замены переменных $x_{j}=E+\beta_{j} / N$ принимает вид

$$
\begin{aligned}
\int_{\mathbb{R}^{3}} \mathrm{~d} \alpha_{1} \mathrm{~d} \alpha_{2} \mathrm{~d} \alpha_{3} O\left(\alpha_{1}, \alpha_{2}, \alpha_{3}\right) \int_{\mathbb{R}^{3}} \mathrm{~d} x_{1} \mathrm{~d} x_{2} \mathrm{~d} x_{3} p_{w, N}^{(3)}\left(x_{1}, x_{2}, x_{3}\right) \\
\times \theta_{\eta}\left(x_{1}-E_{1}\right) \theta_{\eta}\left(x_{2}-E_{2}\right) \theta_{\eta}\left(x_{3}-E_{3}\right),
\end{aligned}
$$


т. е. мы можем записать его в виде интеграла от выражения вида (4.14), для которого пределы с $p_{w, N}$ и $p_{v, N}$ совпадают.

Второй член в правой части равенства (4.15) является пренебрежимо малым. Чтобы убедиться в этом, заметим, что для любой тестовой функции $Q$ мы имеем

$$
\begin{aligned}
\int_{\mathbb{R}^{3}} \mathrm{~d} & \beta_{1} \mathrm{~d} \beta_{2} \mathrm{~d} \beta_{3} Q\left(\beta_{1}, \beta_{2}, \beta_{3}\right) p_{w, N}^{(3)}\left(E+\frac{\beta_{1}}{N}, E+\frac{\beta_{2}}{N}, E+\frac{\beta_{3}}{N}\right) \\
= & \left.N^{3} \int_{\mathbb{R}^{3}} \mathrm{~d} x_{1} \mathrm{~d} x_{2} \mathrm{~d} x_{3} Q\left(N\left(x_{1}-E\right), N x_{2}-E\right), N\left(x_{3}-E\right)\right) p_{w, N}^{(3)}\left(x_{1}, x_{2}, x_{3}\right) \\
& =\left(1-\frac{1}{N}\right)\left(1-\frac{2}{N}\right) \mathrm{E}^{\mathrm{w}} \sum_{i \neq j \neq k} Q\left(N\left(\lambda_{i}-E\right), N\left(\lambda_{j}-E\right), N\left(\lambda_{k}-E\right)\right) .
\end{aligned}
$$

Если бы носитель тестовой функции $Q$ содержался в шаре размера $N^{\varepsilon^{\prime}}$ с $\varepsilon^{\prime}>0$, то этот последний член был бы ограничен сверху величиной

$$
\|Q\|_{\infty} \mathrm{E}^{\mathbf{w}} \mathscr{N}_{C N^{-1+\varepsilon^{\prime}}}^{3}(E) \leqslant C\|Q\|_{\infty} N^{4 \varepsilon^{\prime}} .
$$

Здесь $\mathscr{N}_{\tau}(E)$ обозначает число собственных значений в отрезке $[E-\tau, E+\tau]$, и в этой оценке мы использовали локальный полукруговой закон на отрезках размера $\tau \geqslant N^{-1+\varepsilon^{\prime}}$.

Положим теперь $Q:=O-O_{\eta}$. Из определения $O_{\eta}$ легко вывести, что функция

$$
Q_{1}\left(\beta_{1}, \beta_{2}, \beta_{3}\right)=O\left(\beta_{1}, \beta_{2}, \beta_{3}\right)-O_{\eta}\left(\beta_{1}, \beta_{2}, \beta_{3}\right) \prod_{j=1}^{3} \mathbf{1}\left(\left|\beta_{j}\right| \leqslant N^{\varepsilon^{\prime}}\right)
$$

удовлетворяет оценке $\left\|Q_{1}\right\|_{\infty} \leqslant\|Q\|_{\infty}=\left\|O-O_{\eta}\right\|_{\infty} \leqslant C N \eta=C N^{-\varepsilon}$. Выбирая $\varepsilon^{\prime}<\varepsilon / 4$, мы видим, что вклад функции $Q_{1}$ пренебрежимо мал. Наконец, функция $Q_{2}=Q-Q_{1}$ задается формулой

$$
Q_{2}\left(\beta_{1}, \beta_{2}, \beta_{3}\right)=-O_{\eta}\left(\beta_{1}, \beta_{2}, \beta_{3}\right)\left[1-\prod_{j=1}^{3} \mathbf{1}\left(\left|\beta_{j}\right| \leqslant N^{\varepsilon^{\prime}}\right)\right],
$$

и мы имеем

$$
\begin{aligned}
\left|Q_{2}\right| & \leqslant C\left[\frac{1}{1+\beta_{1}^{2}}\right]\left[\frac{1}{1+\beta_{2}^{2}}\right]\left[\frac{1}{1+\beta_{3}^{2}}\right]\left\{\mathbf{1}\left(\left|\beta_{1}\right| \geqslant N^{\varepsilon^{\prime}}\right)+\cdots\right\} \\
& \leqslant C\left\{N^{-\varepsilon^{\prime}}\left[\frac{N^{\varepsilon^{\prime}}}{N^{2 \varepsilon^{\prime}}+\beta_{1}^{2}}\right]\left[\frac{1}{1+\beta_{2}^{2}}\right]\left[\frac{1}{1+\beta_{3}^{2}}\right]+\cdots\right\} .
\end{aligned}
$$

Следовательно, вклад функции $Q_{2}$ в последний член соотношения (4.17) ограничен сверху величиной

$$
\begin{aligned}
C N^{-3-\varepsilon^{\prime}} \mathrm{E}^{\mathbf{w}} \sum_{i, j, k}\{ & {\left[\frac{N^{-1+\varepsilon^{\prime}}}{N^{-2+2 \varepsilon^{\prime}}+\left(\lambda_{i}-E\right)^{2}}\right]\left[\frac{N^{-1}}{N^{-2}+\left(\lambda_{j}-E\right)^{2}}\right] } \\
& \left.\times\left[\frac{N^{-1}}{N^{-2}+\left(\lambda_{k}-E\right)^{2}}\right]+\cdots\right\} .
\end{aligned}
$$


Тогда из локального полукругового закона, т. е. теоремы 2.5 или теоремы 2.19 , вытекает, что последний член ограничен сверху величиной $N^{-\varepsilon^{\prime}}$ с точностью до некоторого логарифмического множителя. Чтобы убедиться в этом, заметим, что суммы Римана для собственных значений в (4.19) можно заменить на интеграл, так как масштаб разрешения рассматриваемых функций имеет порядок по крайней мере $N^{-1}$. Это завершает доказательство теоремы 4.2.

4.3. Набросок доказательства теоремы 1.7. Мы снова производим интерполяцию между $H$ и $H^{\prime}$ шаг за шагом, заменяя для каждого элемента матрицы $H$ распределение $\nu$ на $\nu^{\prime}$, в соответствии с фиксированным порядком элементов. Пусть $H^{(\tau)}(h)$ - матрица, первые $\tau-1$ элементов которой имеют распределение $\nu^{\prime}$, элемент с номером $\tau$ есть $h$, а оставшиеся элементы имеют распределение $\nu$. Обозначим $i$-е собственное значение матрицы $H^{(\tau)}(h)$ через $\lambda_{i}\left(H^{(\tau)}(h)\right)$. Положим

$$
\mathscr{F}_{\tau}(h):=F\left(N \lambda_{i_{1}}\left(H^{(\tau)}(h)\right), N \lambda_{i_{2}}\left(H^{(\tau)}(h)\right), \ldots\right)
$$

и докажем, что

$$
\left|\mathrm{E} \mathscr{F}_{\tau}(h)-\mathrm{E}^{\prime} \mathscr{F}_{\tau}\left(h^{\prime}\right)\right| \leqslant C N^{-2-c_{0}} .
$$

Так как число шагов замены имеет порядок $N^{2}$, то тем самым мы докажем неравенство (1.77). Будем считать, что $\tau$ представляет $(p q)$-й элемент матрицы, и далее будем опускать индекс $\tau$.

Мы докажем, что

$$
\left|\frac{\partial^{n} \mathscr{F}}{\partial h^{n}}\right| \leqslant C N^{O\left(c_{0}\right)+o(1)}
$$

для любого $n \leqslant 5$. Тогда, используя разложение Тейлора, получим

$$
\mathscr{F}(h)=\sum_{n=0}^{4} \frac{1}{n !} \frac{\partial^{n} \mathscr{F}}{\partial h^{n}}(0) h^{n}+N^{-5 / 2+O\left(c_{0}\right)+o(1)},
$$

так как $|h| \leqslant N^{-5 / 2+o(1)}$ с очень высокой вероятностью. Вычисляя математические ожидания для $h$ по отношению к распределениям $\nu$ и $\nu^{\prime}$ и используя совпадение первых моментов, мы убеждаемся, что вклады членов разложения в (4.22) совпадают, что доказывает неравенство (4.20).

Осталось доказать неравенство (4.21). Предположим для простоты, что $F$ зависит только от одной переменной и $i_{1}=i$. Тогда

$$
\frac{\partial \mathscr{F}}{\partial h}(h)=N F^{\prime}(\lambda) \frac{\partial \lambda_{i}}{\partial h}(h) .
$$

Используя стандартную теорию возмущений первого порядка, мы получаем для $h=h_{p q}$

$$
\frac{\partial \lambda_{i}}{\partial h}=2 \operatorname{Re} u_{i}(p) \bar{u}_{i}(q)
$$

где $\mathbf{u}_{i}=\left(u_{i}(1), \ldots, u_{i}(N)\right)$ - собственная функция, отвечающая собственному значению $\lambda_{i}$. Так как собственные векторы делокализованы, мы имеем

$$
\|\mathbf{u}\|_{\infty} \approx N^{-1 / 2}
$$


(по модулю логарифмических поправок, см. (1.31)), откуда вытекает

$$
\left|\frac{\partial \lambda_{i}}{\partial h}\right| \lesssim O\left(N^{-1}\right)
$$

Следовательно,

$$
\left|\frac{\partial \mathscr{F}}{\partial h}\right| \lesssim N N^{c_{0}} N^{-1}=N^{c_{0}} .
$$

Чтобы получить формулы для производных высших порядков, нам также понадобится дифференцировать собственные функции. Это приводит к резонансам, например, при $h=h_{p q}$ имеем

$$
\frac{\partial u_{i}(p)}{\partial h_{p q}}=\sum_{j \neq i} \frac{1}{\lambda_{i}-\lambda_{j}} u_{j}(p)\left[\bar{u}_{j}(p) u_{i}(q)+\bar{u}_{j}(q) u_{i}(p)\right] .
$$

Следовательно,

$$
\left|\frac{\partial u_{i}(p)}{\partial h}\right| \leqslant N^{-1 / 2} \frac{1}{N} \sum_{j \neq i} \frac{1}{\left|\lambda_{i}-\lambda_{j}\right|} \lesssim N^{-1 / 2+c_{0}},
$$

в предположении, что собственные значения регулярно следуют полукруговому закону и что никакие два соседних собственных значения не находятся на расстоянии меньше $N^{-1-c_{0}}$ (см. (1.78)). Подставляя эту оценку в производную формулы (4.23), мы получаем

$$
\left|\frac{\partial^{2} \lambda_{i}}{\partial h^{2}}\right| \lesssim C N^{-1+c_{0}}
$$

Используя последнюю оценку и $(4.25)$ и восстанавливая общий случай, когда $F$ зависит от более чем одной переменной, мы получаем

$$
\left|\frac{\partial^{2} \mathscr{F}}{\partial h^{2}}\right| \lesssim C N\left|\partial_{i i} F\right|\left|\partial^{2} \lambda_{i}\right|+C N^{2}\left|\partial_{i j} F\right|\left|\partial \lambda_{j}\right|\left|\partial \lambda_{i}\right| \leqslant C N^{2 c_{0}} .
$$

Рассуждение для высших производных аналогично. Основными техническими средствами являются делокализационная оценка на собственные векторы (4.24), которая может быть получена из локального полукругового закона, и оценка на нижние хвосты (1.78).

\section{5. Универсальность для матриц Вигнера: сведение компонент доказательства воедино}

В этом разделе мы сводим воедино полученную выше информацию и доказываем наш основной результат - теорему 5.1. Мы приведем формулировку нашего наиболее общего результата из [8]. Тот же результат при несколько более сильных ограничениях был получен в наших предыдущих работах, например, [16; теорема 2.3], [6; теорема 3.1] и [9; теорема 2.2].

Напомним, что $p_{N}\left(\lambda_{1}, \ldots, \lambda_{N}\right)$ обозначает симметрическую совместную плотность собственных значений $(N \times N)$-матрицы Вигнера $H$. Для простоты мы 
будем использовать формализм, при котором совместное распределение собственных значений предполагается абсолютно непрерывным относительно меры Лебега, однако это не является обязательным для доказательства. Напомним также определение $k$-точечных корреляционных функций (маргиналов) $p_{N}^{(k)}$ из (1.34). Для корреляционных функций ансамблей ГУА и ГОА будут использоваться обозначения $p_{N, \Gamma у А}^{(k)}$ и $p_{N, \Gamma О А}^{(k)}$.

Мы будем рассматривать корреляционные функции вблизи фиксированного уровня энергии $E$ в нормировке, при которой локальная плотность равна единице. Универсальность синус-ядра для ансамбля ГУА утверждает, что нормированные корреляционные функции слабо сходятся к определителю синус-ядра $K(x)=\frac{\sin \pi x}{\pi x}$, т. е.

$$
\frac{1}{\left[\varrho_{\mathrm{sc}}(E)\right]^{k}} p_{N, \Gamma \mathrm{IA}}^{(k)}\left(E+\frac{\alpha_{1}}{N \varrho_{\mathrm{sc}}(E)}, \ldots, E+\frac{\alpha_{k}}{N \varrho_{\mathrm{sc}}(E)}\right) \rightarrow \operatorname{det}\left(K\left(\alpha_{\ell}-\alpha_{j}\right)\right)_{\ell, j=1}^{k}
$$

при $N \rightarrow \infty$ для любой фиксированной энергии $|E|<2$ внутри спектра [77], [2]. Аналогичные результаты имеют место и для случая ГОА; синус-ядро здесь заменяется на аналогичную, но несколько более сложную функцию (см. [1]). Наш основной результат утверждает, что универсальность (5.1) имеет место для эрмитовых или симметрических обобщенных матриц Вигнера после небольшого усреднения по энергии $E$.

Теорема 5.1 [8; теорема 2.2]. Пусть $H$ - симметрическая или эрмитова обобщенная матрица Вигнера размера $N \times N$. В эрмитовом случае мы предполагаем, что вещественная и мнимая части являются независимыми одинаково распределенными величинами. Предположим, что распределение $\nu$ нормированных матричных элементов $\sqrt{N} h_{i j}$ имеет субэкспоненциальное убъвание (2.32). Пусть $k \geqslant 1$ и $O: \mathbb{R}^{k} \rightarrow \mathbb{R}$ - непрерьвная функция с компактным носителем. Тогда для любого $|E|<2$ мы имеем

$$
\begin{aligned}
\lim _{b \rightarrow 0} & \lim _{N \rightarrow \infty} \frac{1}{2 b} \int_{E-b}^{E+b} \mathrm{~d} v \int_{\mathbb{R}^{k}} \mathrm{~d} \alpha_{1} \cdots \mathrm{d} \alpha_{k} O\left(\alpha_{1}, \ldots, \alpha_{k}\right) \\
& \times \frac{1}{\left[\varrho_{\mathrm{sc}}(v)\right]^{k}}\left(p_{N}^{(k)}-p_{N, \#}^{(k)}\right)\left(v+\frac{\alpha_{1}}{N \varrho_{\mathrm{sc}}(v)}, \ldots, v+\frac{\alpha_{k}}{N \varrho_{\mathrm{sc}}(v)}\right)=0
\end{aligned}
$$

где \# обозначает ГОА или ГУА для симметрического или эрмитова случаев соответственно.

ДокАЗАТЕЛЬство. Для определенности будем рассматривать симметрический случай, т. е. предел будет гауссовым ортогональным ансамблем (ГОА), соответствующим параметру $\beta=1$ в общем формализме. Совместное распределение собственных значений $\mathbf{x}=\left(x_{1}, \ldots, x_{N}\right)$ задается следующей мерой:

$$
\mu=\mu_{N}(\mathrm{~d} \mathbf{x})=\frac{e^{-N \mathscr{H}(\mathbf{x})}}{Z} \mathrm{~d} \mathbf{x}, \quad \mathscr{H}(\mathbf{x})=\sum_{i=1}^{N} \frac{x_{i}^{2}}{4}-\frac{1}{N} \sum_{i<j} \log \left|x_{j}-x_{i}\right|,
$$

и мы предполагаем, что собственные значения упорядочены, т. е. мера $\mu$ ограничивается на подмножество $\Sigma_{N}=\left\{\mathbf{x} \in \mathbb{R}^{N}: x_{1}<\cdots<x_{N}\right\}$. 
Пусть $\widehat{H}$ - симметрическая матрица Вигнера, распределение отдельных элементов которой удовлетворяет условию субэкспоненциального убывания (2.32). Рассмотрим эволюцию матрицы в соответствии с матричнозначным процессом Орнштейна-Уленбека (1.54):

$$
\mathrm{d} H_{t}=\frac{1}{\sqrt{N}} \mathrm{~d} \beta_{t}-\frac{1}{2} H_{t} \mathrm{~d} t, \quad H_{0}=\widehat{H},
$$

и напомним, что распределение для $H_{t}$ при каждом фиксированном $t>0$ совпадает с

$$
e^{-t / 2} \widehat{H}+\left(1-e^{-t}\right)^{1 / 2} V
$$

где $V$ - независимая матрица ГОА. Распределение $\nu_{t}(\mathrm{~d} x)=u_{t}(x) \mathrm{d} x$ матричных элементов эволюционирует в соответствии с процессом Орнштейна-Уленбека на $\mathbb{R}$, т. е.

$$
\partial_{t} u_{t}=A u_{t}, \quad A=\frac{1}{2} \frac{\partial^{2}}{\partial x^{2}}-\frac{x}{2} \frac{\partial}{\partial x} .
$$

Заметим, что начальное распределение $\nu=\nu_{0}$ может иметь особенности, но при любом $t>0$ распределение $\nu_{t}$ является абсолютно непрерывным.

Процесс Орнштейна-Уленбека (5.5) индуцирует броуновское движение Дайсона собственных значений [15], с порождающим оператором, задаваемым формулой

$$
L=\sum_{i=1}^{N} \frac{1}{2 N} \partial_{i}^{2}+\sum_{i=1}^{N}\left(-\frac{1}{4} x_{i}+\frac{1}{2 N} \sum_{j \neq i} \frac{1}{x_{i}-x_{j}}\right) \partial_{i},
$$

действующим на $L^{2}(\mu)$. Мера $\mu$ является инвариантной и обратимой по отношению к динамике, порождаемой оператором $L$.

Обозначим распределение собственных значений в момент времени $t$ через $f_{t}(\mathbf{x}) \mu(\mathrm{d} \mathbf{x})$. Тогда функция $f_{t}$ удовлетворяет уравнению

$$
\partial_{t} f_{t}=L f_{t}
$$

с начальным условием $f_{0}$, задаваемым плотностью собственных значений матрицы Вигнера $\widehat{H}$. Используя предыдущие обозначения, мы имеем $p_{N}=f_{0} \mu_{N}$, где функция $p_{N}$, а значит и $f_{0}$, может быть сингулярной по отношению к мере Лебега. В силу условия $\beta \geqslant 1$, собственные значения не перекрываются, т. е. динамика (5.7) корректно определена на множестве $\Sigma_{N}$. Используя непосредственную симметризацию, можно продолжить равновесную меру, функции плотности и динамику на все пространство $\mathbb{R}^{N}$. Мы будем использовать упорядоченные собственные значения везде кроме определения корреляционных функций (1.34), где симметрическая версия более удобна. Данное различие не будет приниматься во внимание в дальнейшем.

Теорема 5.1 была вначале доказана в [16] для стандартных матриц Вигнера и при более сильных ограничениях на распределение отдельных элементов. Здесь мы изложим более прямое доказательство, следуя [9], но для простоты обозначений будем рассматривать лишь случай матриц Вигнера. Основная часть доказательства теоремы 5.1 состоит из следующих трех шагов. 
Шаг 1. Вначале мы покажем, что существует $\varepsilon_{0}>0$ такое, что корреляционные функции любого ансамбля Вигнера с гауссовой конволюцией с дисперсией $t \sim N^{-\varepsilon_{0}}$ совпадают с ГОА. Другими словами, любой ансамбль вида (5.4) с $t \geqslant N^{-\varepsilon_{0}}$ (и субэкспоненциальным убыванием элементов матрицы $\widehat{H}$ ) имеет универсальную локальную статистику.

Шаг 2. Положим $t=N^{-\varepsilon_{0}}$. Тогда мы покажем, что для любой данной матрицы Вигнера $H$ можно найти другую матрицу Вигнера $\widehat{H}$ такую, что первые три момента матриц $H$ и $H_{t}=e^{-t / 2} \widehat{H}+\left(1-e^{-t}\right)^{1 / 2} V$ совпадают, а четвертые моменты являются близкими порядка $O\left(N^{-\varepsilon_{0}}\right)$.

Шаг 3. Теорема 4.2, которая была следствием теоремы сравнения для функций Грина, показывает, что локальные корреляционные функции матриц $H$ и $H_{t}$ из шага 2 совпадают. Вместе с результатом шага 1 это завершит доказательство теоремы 5.1 .

Теперь мы сформулируем утверждения шагов 1 и 2 более точно. Шаг 3 уже завершен.

5.1. Шаг 1: универсальность для гауссовых конволюций. Этот шаг заключается в применении теоремы 3.1, которую мы сформулируем здесь для нашего частного случая.

ТЕОРема 5.2. Предположим, что распределение вероятностей начальной симметрической матриць Вигнера $\widehat{H}$ имеет субэкспоненииальное убывание (2.32) с некоторым показателем $\vartheta$, и пусть матрицы $H_{t}$ задаются гауссовыми конволюциями (5.4). Пусть $p_{t, N}^{(k)}$ обозначает $k$-точечную корреляционную функиию собственнъх значений матрицы $H_{t}$. Тогда существует такое $\varepsilon_{0}>0$, зависящее от параметров в $(2.32)$, что для любого $t \geqslant N^{-\varepsilon_{0}}$ мы имеем

$$
\begin{aligned}
\lim _{b \rightarrow 0} & \lim _{N \rightarrow \infty} \frac{1}{2 b} \int_{E-b}^{E+b} \mathrm{~d} v \int_{\mathbb{R}^{k}} \mathrm{~d} \alpha_{1} \cdots \mathrm{d} \alpha_{k} O\left(\alpha_{1}, \ldots, \alpha_{k}\right) \\
& \times \frac{1}{\left[\varrho_{\mathrm{sc}}(v)\right]^{k}}\left(p_{t, N}^{(k)}-p_{N, \Gamma \mathrm{OA}}^{(k)}\right)\left(v+\frac{\alpha_{1}}{N \varrho_{\mathrm{sc}}(v)}, \ldots, v+\frac{\alpha_{k}}{N \varrho_{\mathrm{sc}}(v)}\right)=0
\end{aligned}
$$

для любой непрерывной функиии $О$ с компактным носителем.

Заметим, что пороговый показатель $\varepsilon_{0}$ может быть задан явно. Если использовать локальный полукруговой закон из теоремы 2.5 и его следствие теорему 2.7, то в качестве $\varepsilon_{0}$ можно взять любое число, меньшее $1 / 7$. При использовании сильного локального полукругового закона - теоремы 2.19 в качестве показателя $\varepsilon_{0}$ можно взять любое число, меньшее 1.

ДокАзАТЕЛЬство. Нам необходимо лишь проверить, что выполнены предположения теоремы 3.1. Вначале заметим, что гамильтониан равновесной меры задается формулой (5.3), и он очевидно имеет вид (3.5), так что условие I выполнено автоматически. Условие на энтропию (3.11) для начального состояния $f_{0}$ может быть и не выполнено, так как функция $f_{0}$ может быть даже сингулярной. Тем не менее, в силу свойства полугруппы для потока Орнштейна-Уленбека, мы можем рассмотреть начальное условие $f_{t_{0}}$ для потока $f_{t}, t \geqslant t_{0}$, при некотором $t_{0} \leqslant N^{-\varepsilon_{0}}$, так как в формулировке теоремы 3.1 
рассматривается лишь время $t \geqslant N^{-\varepsilon_{0}}$. Поэтому достаточно показать, что условие на энтропию выполнено для некоторого достаточно малого $t_{0} \ll N^{-\varepsilon_{0}}$.

Пусть $\nu_{t}$ обозначает распределение отдельных элементов матрицы $H_{t}$, а $\bar{\nu}_{t}-$ вероятностную меру матрицы $H_{t}$. Пусть $\bar{\nu}_{\Gamma О А}$ обозначает вероятностную меру

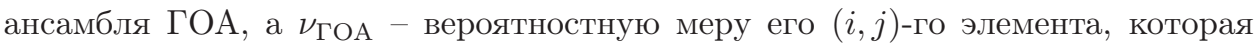
является гауссовой мерой с нулевым математическим ожиданием и дисперсией $1 / N$. Так как динамики матричных элементов независимы (помимо условия симметрии), а энтропия аддитивна, мы имеем соотношение

$$
\int \log \left(\frac{\mathrm{d} \bar{\nu}_{t}}{\mathrm{~d} \bar{\nu}_{\Gamma \mathrm{OA}}}\right) \mathrm{d} \bar{\nu}_{t}=\sum_{i \leqslant j} \int \log \left(\frac{\mathrm{d} \nu_{t}}{\mathrm{~d} \nu_{\Gamma \mathrm{OA}}}\right) \mathrm{d} \nu_{t} \leqslant C N^{2} \int \log \left(\frac{\mathrm{d} \nu_{t}}{\mathrm{~d} \nu_{\Gamma \mathrm{OA}}}\right) \mathrm{d} \nu_{t}
$$

поскольку суммирование производится по индексам всех независимых элементов $1 \leqslant i \leqslant j \leqslant N$. Очевидно, что процесс $t \rightarrow \nu_{t}$ является процессом Орнштейна-Уленбека, а каждый член энтропии в правой части соотношения (5.9) ограничен сверху числом $C N$, при условии, что $t \geqslant t_{0}:=1 / N$ и $\nu_{0}$ имеет субэкспоненциальное убывание. Так как энтропия маргинального распределения на собственных значениях ограничена сверху энтропией тотальной меры на матрице, нами доказано неравенство

$$
\int f_{1 / N} \log f_{1 / N} \mathrm{~d} \mu \leqslant C N^{3}
$$

что дает неравенство (3.11). Следовательно, чтобы применить теорему 3.1, необходимо лишь проверить выполнение условий II, III и IV. Очевидно, что условия II и IV вытекают из локального полукругового закона - теоремы 2.5 с $\varrho(E)=\varrho_{\mathrm{sc}}(E)$ (заметим, что в случае ограниченной дисперсии мы имеем $M \sim N)$, а условие III было доказано в теореме 2.7. Теперь мы можем применить теорему 3.1, получив соотношение (5.8) с любым $\varepsilon_{0}<\varepsilon$, где $\varepsilon$ взято из теоремы 2.7 , т. е. $\varepsilon_{0}$ может быть любым числом, меньшим $1 / 7$. Если же применить сильный локальный полукруговой закон - теорему 2.19 , то из (2.114) мы получим

$$
\mathrm{E} \sum_{j}\left(\lambda_{j}-\gamma_{j}\right)^{2} \lesssim N^{-1}
$$

т. е. условие III (неравенство (3.9)) выполнено для любого $\mathfrak{a}<1 / 2$, и поэтому $\varepsilon_{0}$ может быть любым числом, меньшим 1. Теорема доказана.

5.2. Шаг 2: лемма о совпадении. Для любой вещественной случайной величины $\xi$ обозначим через $m_{k}(\xi)=\mathrm{E} \xi^{k}$ ее $k$-й момент. В силу неравенства Коши-Буняковского, последовательность моментов $m_{1}, m_{2}, \ldots$ не может быть произвольной, например, мы имеем $\left|m_{1}\right|^{k} \leqslant m_{k}, m_{2}^{2} \leqslant m_{4}$ и т. д. Кроме того, имеются и более тонкие неравенства. Например, если $m_{1}=0$, то

$$
m_{4} m_{2}-m_{3}^{2} \geqslant m_{2}^{3}
$$

Это может быть получено из неравенства

$$
m_{3}^{2}=\left[\mathrm{E} \xi^{3}\right]^{2}=\left[\mathrm{E} \xi\left(\xi^{2}-1\right)\right]^{2} \leqslant\left[\mathrm{E} \xi^{2}\right]\left[\mathrm{E}\left(\xi^{2}-1\right)^{2}\right]=m_{2}\left(m_{4}-2 m_{2}^{2}+1\right)
$$


и того факта, что неравенство (5.11) не зависит от масштаба, т. е. его достаточно доказать для $m_{2}=1$. Легко видеть, что (5.11) обращается в равенство тогда и только тогда, когда носитель величины $\xi$ состоит в точности из двух точек (за исключением тривиального случая $\xi \equiv 0$ ).

Это ограничение показывает, что для данной последовательности четырех допустимых моментов $m_{1}=0, m_{2}=1, m_{3}, m_{4}$ может не существовать гауссово делимой случайной величины $\xi$ с такими моментами. Например, последовательность моментов $\left(m_{1}, m_{2}, m_{3}, m_{4}\right)=(0,1,0,1)$ однозначно характеризует стандартную бернуллиевскую случайную величину (т. е. $\xi= \pm 1$ с вероятностями $1 / 2,1 / 2)$. Тем не менее, если позволить четвертому моменту изменяться в некотором небольшом интервале, то можно найти малую гауссову конволюцию с данными четырьмя допустимыми моментами. Это и утверждается в следующей лемме, которая завершит доказательство шага 2.

Лемма 5.3 [9; лемма 6.5]. Пусть $m_{3}$ и $m_{4}$ - два вещественных числа, удовлетворяющие неравенствам

$$
m_{4}-m_{3}^{2}-1 \geqslant 0, \quad m_{4} \leqslant C_{2}
$$

для некоторой положительной константы $C_{2}$. Пусть $\xi^{\mathrm{G}}-$ гауссова случайная величина с математическим ожиданием 0 и дисперсией 1. Тогда для любого достаточно малого $\gamma>0$ (зависящего от $C_{2}$ ) существует вещественная случайная величина $\xi_{\gamma}$ с субэкспоненииальным убыванием и независимая от $\xi^{\mathrm{G}}$, такал, что первые четыре момента величинь

$$
\xi^{\prime}=(1-\gamma)^{1 / 2} \xi_{\gamma}+\gamma^{1 / 2} \xi^{\mathrm{G}}
$$

cymъ $m_{1}\left(\xi^{\prime}\right)=0, m_{2}\left(\xi^{\prime}\right)=1, m_{3}\left(\xi^{\prime}\right)=m_{3}$ u $m_{4}\left(\xi^{\prime}\right)$, причем

$$
\left|m_{4}\left(\xi^{\prime}\right)-m_{4}\right| \leqslant C \gamma
$$

для некоторого $C$, зависящего от $C_{2}$.

ДокАЗАТЕЛЬСТвО. При помощи непосредственной конструкции легко убедиться в справедливости следующего утверждения.

УТВЕРЖДЕНИЕ 5.4. Для любъх чисел $m_{3}, m_{4}$, удовлетворяющих неравенству $m_{4}-m_{3}^{2}-1 \geqslant 0$, существует случайная величина $X$ с первыми четырьмя моментами, равными $0,1, m_{3}, m_{4}$, имеющая субэкспоненииальное убъвание.

Пусть $\zeta$ - вещественная случайная величина, независимая от $\xi^{\mathrm{G}}$ и имеющая первые четыре момента $0,1, m_{3}(\zeta)$ и $m_{4}(\zeta)<\infty$. Тогда первые четыре момента величины

$$
\zeta^{\prime}=(1-\gamma)^{1 / 2} \zeta+\gamma^{1 / 2} \xi^{\mathrm{G}}
$$

суть 0,1 ,

$$
m_{3}\left(\zeta^{\prime}\right)=(1-\gamma)^{3 / 2} m_{3}(\zeta)
$$

и

$$
m_{4}\left(\zeta^{\prime}\right)=(1-\gamma)^{2} m_{4}(\zeta)+6 \gamma-3 \gamma^{2}
$$


Используя утверждение 5.4, мы получаем, что для любого $\gamma>0$ существует вещественная случайная величина $\xi_{\gamma}$, первые четыре момента которой суть 0,1 ,

$$
m_{3}\left(\xi_{\gamma}\right)=(1-\gamma)^{-3 / 2} m_{3}
$$

и

$$
m_{4}\left(\xi_{\gamma}\right)=m_{3}\left(\xi_{\gamma}\right)^{2}+\left(m_{4}-m_{3}^{2}\right) .
$$

Из $m_{4} \leqslant C_{2}$ вытекает, что $m_{3}^{2} \leqslant C_{2}^{3 / 2}$, откуда

$$
\left|m_{4}\left(\xi_{\gamma}\right)-m_{4}\right| \leqslant C \gamma
$$

для некоторого $C$, зависящего от $C_{2}$. Отсюда и из (5.16) и (5.17) мы получаем, что $\xi^{\prime}=(1-\gamma)^{1 / 2} \xi_{\gamma}+\gamma^{1 / 2} \xi^{\mathrm{G}}$ удовлетворяет соотношению $m_{3}\left(\xi^{\prime}\right)=m_{3}$ и неравенству (5.14). Это завершает доказательство леммы 5.3.

\section{Приложение А. Оценки больших уклонений: доказательство леммы 2.12}

Оценки из леммы 2.12 слабее, чем соответствующие результаты Хансона и Райта [101], использованные в [57], [6], однако они верны для независимых, но не обязательно одинаково распределенных случайных величин с субэкспоненциальным убыванием; более того, доказательства намного проще. Таким образом, требование гауссова убывания у Хансона и Райта в нашем случае ослаблено до субэкспоненциального убывания, но оценка вероятностей хвостов слабее.

ДокАЗАТЕЛЬСтво НеРАВеНСтвА (2.68). Не ограничивая общности, мы можем предположить, что $\sigma=1$. Из условия $(2.67)$ вытекает, что для $k$-го момента величины $a_{i}$ имеется следующая оценка:

$$
\mathrm{E}\left|a_{i}\right|^{k} \leqslant(C k)^{\alpha k}
$$

для некоторого $C>0$.

Вначале для каждого $p \in \mathbb{N}$ оценим величину

$$
\mathrm{E}\left|\sum_{i=1}^{N} a_{i} A_{i}\right|^{p}
$$

Из неравенства Марцинкевича-Зигмунда для $p \geqslant 2$ мы получаем

$$
\mathrm{E}\left|\sum_{i} a_{i} A_{i}\right|^{p} \leqslant(C p)^{p / 2} \mathrm{E}\left(\sum_{i}\left|a_{i} A_{i}\right|^{2}\right)^{p / 2}
$$

(оценку константы можно найти, например, в [107; упражнение 2.2.30]). Подставляя (А.1) в (А.3), мы получаем

$$
\mathrm{E}\left|\sum_{i} a_{i} A_{i}\right|^{p} \leqslant\left(C p^{\frac{1}{2}+\alpha}\right)^{p}\left(\sum_{i}\left|A_{i}\right|^{2}\right)^{p / 2} .
$$

Теперь неравенство (2.68) получается подстановкой $p=\log N$ и применением неравенства Маркова для высших моментов. 
ДОКАЗАТЕЛЬСТВо НЕРАВЕНСТВА (2.69). Заметим, что случайные величины $\left|a_{i}\right|^{2}-1 \quad(1 \leqslant i \leqslant N)$ являются независимыми с математическим ожиданием 0 и дисперсиями, ограниченными некоторой константой $C$. Более того, для $k$-го момента величины $\left|a_{i}\right|^{2}-1$ имеется следующая верхняя оценка:

$$
\mathrm{E}\left(\left|a_{i}\right|^{2}-1\right)^{k} \leqslant(C k)^{2 \alpha k} .
$$

Тогда, повторяя доказательство неравенства (2.68) с заменой $a_{i}$ на $\left|a_{i}\right|^{2}-1$, мы получим неравенство (2.69).

ДоКАЗАТЕЛЬСтво неРАвенСтвА (2.70). Для любого $p \in \mathbb{N}, p \geqslant 2$, оценим величину

$$
\mathrm{E}\left|\sum_{i>j} \bar{a}_{i} \xi_{i}\right|^{p} \equiv \mathrm{E}\left|\sum_{i>j} \bar{a}_{i} B_{i j} a_{j}\right|^{p}
$$

где $\xi_{i}:=\sum_{j<i} B_{i j} a_{j}$. Заметим, что величины $a_{i}$ и $\xi_{i}$ являются независимыми для любого фиксированного $i$. По определению,

$$
X_{n} \equiv \sum_{i=1}^{n} \bar{a}_{i} \xi_{i}
$$

является мартингалом. Используя неравенство Буркхольдера, мы получаем

$$
\mathrm{E}\left|\sum_{i} \bar{a}_{i} \xi_{i}\right|^{p} \leqslant(C p)^{3 p / 2} \mathrm{E}\left(\sum_{i}\left|\bar{a}_{i} \xi_{i}\right|^{2}\right)^{p / 2}
$$

(информацию о константе можно найти в [108; раздел VII.3]). Используя обобщенное неравенство Минковского, независимость величин $a_{i}$ и $\xi_{i}$, а также неравенство (А.1), мы получаем

$$
\begin{aligned}
{\left[\mathrm{E}\left(\sum_{i}\left|\bar{a}_{i} \xi_{i}\right|^{2}\right)^{p / 2}\right]^{2 / p} } & \leqslant \sum_{i}\left[\mathrm{E}\left|\bar{a}_{i} \xi_{i}\right|^{p}\right]^{2 / p}=\sum_{i}\left[\mathrm{E}\left(\left|\bar{a}_{i}\right|^{p}\right) \mathrm{E}\left(\left|\xi_{i}\right|^{p}\right)\right]^{2 / p} \\
& \leqslant(C p)^{2 \alpha} \sum_{i}\left[\mathrm{E}\left(\left|\xi_{i}\right|^{p}\right)\right]^{2 / p}
\end{aligned}
$$

Из (А.4) вытекает, что

$$
\mathrm{E}\left(\left|\xi_{i}\right|^{p}\right) \leqslant\left(C p^{1 / 2+\alpha}\right)^{p}\left(\sum_{j}\left|B_{i j}\right|^{2}\right)^{p / 2} .
$$

Отсюда и из (А.8) мы получаем

$$
\mathrm{E}\left|\sum_{i} \bar{a}_{i} \xi_{i}\right|^{p} \leqslant(C p)^{2 p(1+\alpha)}\left(\sum_{i} \sum_{j}\left|B_{i j}\right|^{2}\right)^{p / 2} .
$$

Полагая $p=\log N$ и применяя неравенство Маркова, мы получаем неравенство (2.70). 


\section{Приложение В. Доказательство теоремы 3.1}

Вспомнив обозначения, введенные в (3.56) и рядом с этими формулами, мы начнем с тождества (3.55):

$$
\begin{gathered}
\int_{E-b}^{E+b} \frac{\mathrm{d} E^{\prime}}{2 b} \int_{\mathbb{R}^{n}} \mathrm{~d} \alpha_{1} \cdots \mathrm{d} \alpha_{n} O\left(\alpha_{1}, \ldots, \alpha_{n}\right) p_{\tau, N}^{(n)}\left(E^{\prime}+\frac{\alpha_{1}}{N \varrho(E)}, \ldots, E^{\prime}+\frac{\alpha_{n}}{N \varrho(E)}\right) \\
=\int_{E-b}^{E+b} \frac{\mathrm{d} E^{\prime}}{2 b} \sum_{\mathbf{m} \in S_{n}} \sum_{i=1}^{N} Y_{i, \mathbf{m}}\left(E^{\prime}, \mathbf{x}\right) .
\end{gathered}
$$

Нам необходимо показать, что

$$
\lim _{N \rightarrow \infty}\left|\int_{E-b}^{E+b} \frac{\mathrm{d} E^{\prime}}{2 b} \int \sum_{\mathbf{m} \in S_{n}} \sum_{i=1}^{N} Y_{i, \mathbf{m}}\left(E^{\prime}, \mathbf{x}\right)\left(f_{\tau}-1\right) \mathrm{d} \mu\right|=0 .
$$

Пусть $M$ - параметр, зависящий от $N$, который будет выбран в конце доказательства. Положим

$$
S_{n}(M):=\left\{\mathbf{m} \in S_{n}: m_{n} \leqslant M\right\}, \quad S_{n}^{c}(M):=S_{n} \backslash S_{n}(M)
$$

и заметим, что $\left|S_{n}(M)\right| \leqslant M^{n-1}$. Для доказательства равенства (В.2) достаточно установить, что справедливо равенство

$$
\lim _{N \rightarrow \infty}\left|\int_{E-b}^{E+b} \frac{\mathrm{d} E^{\prime}}{2 b} \int \sum_{\mathbf{m} \in S_{n}(M)} \sum_{i=1}^{N} Y_{i, \mathbf{m}}\left(E^{\prime}, \mathbf{x}\right)\left(f_{\tau}-1\right) \mathrm{d} \mu\right|=0,
$$

а равенство

$$
\lim _{N \rightarrow \infty} \sum_{\mathbf{m} \in S_{n}^{c}(M)}\left|\int_{E-b}^{E+b} \frac{\mathrm{d} E^{\prime}}{2 b} \int \sum_{i=1}^{N} Y_{i, \mathbf{m}}\left(E^{\prime}, \mathbf{x}\right) f_{\tau} \mathrm{d} \mu\right|=0
$$

выполнено для любого $\tau>N^{-2 \varepsilon+\delta}$ (заметим, что значение $\tau=\infty$ соответствует равновесному состоянию, т. е. $\left.f_{\infty}=1\right)$.

Шаг 1: случай малого $\mathbf{m}$; доказательство равенства (В.3). После интегрирования по $\mathrm{d} E^{\prime}$ мы можем применить теорему 3.3 к функции

$$
G\left(u_{1}, u_{2}, \ldots\right):=\int_{\mathbb{R}} \widetilde{O}\left(y, u_{1}, u_{2}, \ldots\right) \mathrm{d} y,
$$

т. е. к величине

$$
\int_{\mathbb{R}} \mathrm{d} E^{\prime} Y_{i, \mathbf{m}}\left(E^{\prime}, \mathbf{x}\right)=\frac{1}{N} G\left(N\left(x_{i}-x_{i+m_{2}}\right), \ldots\right)
$$

для любых фиксированных $i$ и $\mathbf{m}$.

Для любых $E$ и $0<\xi<b$ определим наборы целых чисел $J=J_{E, b, \xi}$ и $J^{ \pm}=J_{E, b, \xi}^{ \pm}$формулами

$$
J:=\left\{i: \gamma_{i} \in[E-b, E+b]\right\}, \quad J^{ \pm}:=\left\{i: \gamma_{i} \in[E-(b \pm \xi), E+b \pm \xi]\right\}
$$


где $\gamma_{i}$ были введены в (3.8). Очевидно, что $J^{-} \subset J \subset J^{+}$. Используя эти обозначения, мы имеем

$$
\int_{E-b}^{E+b} \frac{\mathrm{d} E^{\prime}}{2 b} \sum_{i=1}^{N} Y_{i, \mathbf{m}}\left(E^{\prime}, \mathbf{x}\right)=\int_{E-b}^{E+b} \frac{\mathrm{d} E^{\prime}}{2 b} \sum_{i \in J^{+}} Y_{i, \mathbf{m}}\left(E^{\prime}, \mathbf{x}\right)+\Omega_{J, \mathbf{m}}^{+}(\mathbf{x}) .
$$

Поправочный член $\Omega_{J, \mathbf{m}}^{+}$, неявно определяемый соотношением (B.6), происходит из тех индексов $i \notin J^{+}$, для которых $x_{i} \in[E-b, E+b]+O\left(N^{-1}\right)$, так как $Y_{i, \mathbf{m}}\left(E^{\prime}, \mathbf{x}\right)=0$, если не выполнено условие $\left|x_{i}-E^{\prime}\right| \leqslant C / N$ (константа зависит от носителя функции $O)$. Следовательно,

$$
\left|\Omega_{J, \mathbf{m}}^{+}(\mathbf{x})\right| \leqslant C N^{-1} b^{-1} \#\left\{i:\left|x_{i}-\gamma_{i}\right| \geqslant \xi / 2\right\}
$$

для любого достаточно большого $N$, при условии $\xi \gg 1 / N$, где мы также использовали ограниченность функции $O$. Дополнительный множитель $N^{-1}$ происходит из интегрирования по $\mathrm{d} E^{\prime}$. Переходя к математическому ожиданию по отношению к мере $f_{\tau} \mathrm{d} \mu$, мы получаем

$$
\int\left|\Omega_{J, \mathbf{m}}^{+}(\mathbf{x})\right| f_{\tau} \mathrm{d} \mu \leqslant C b^{-1} \xi^{-2} N^{-1} \int \sum_{i}\left(x_{i}-\gamma_{i}\right)^{2} f_{\tau} \mathrm{d} \mu=C b^{-1} \xi^{-2} N^{-1-2 \mathfrak{a}}
$$

где мы воспользовались условием III (см. (3.9)). Кроме того, справедлива оценка

$$
\begin{aligned}
\int_{E-b}^{E+b} & \frac{\mathrm{d} E^{\prime}}{2 b} \sum_{i \in J^{+}} Y_{i, \mathbf{m}}\left(E^{\prime}, \mathbf{x}\right) \leqslant \int_{E-b}^{E+b} \frac{\mathrm{d} E^{\prime}}{2 b} \sum_{i \in J^{-}} Y_{i, \mathbf{m}}\left(E^{\prime}, \mathbf{x}\right)+C N^{-1}\left|J^{+} \backslash J^{-}\right| \\
& =\int_{\mathbb{R}} \frac{\mathrm{d} E^{\prime}}{2 b} \sum_{i \in J^{-}} Y_{i, \mathbf{m}}\left(E^{\prime}, \mathbf{x}\right)+C(N b)^{-1}\left|J^{+} \backslash J^{-}\right|+\Xi_{J, \mathbf{m}}^{+}(\mathbf{x}) \\
& \leqslant \int_{\mathbb{R}} \frac{\mathrm{d} E^{\prime}}{2 b} \sum_{i \in J} Y_{i, \mathbf{m}}\left(E^{\prime}, \mathbf{x}\right)+C(N b)^{-1}\left|J^{+} \backslash J^{-}\right|+C(N b)^{-1}\left|J \backslash J^{-}\right|+\Xi_{J, \mathbf{m}}^{+}(\mathbf{x}),
\end{aligned}
$$

где поправочный член $\Xi_{J, \mathbf{m}}^{+}$, определяемый соотношением (В.8), происходит из тех индексов $i \in J^{-}$, для которых $x_{i} \notin[E-b, E+b]+O(1 / N)$. Он удовлетворяет той же оценке (В.7), что и $\Omega_{J, \mathbf{m}}^{+}$. Из непрерывности функции $\varrho$ вытекает, что плотность величины $\gamma_{i}$ ограничена сверху числом $C N$, откуда $\left|J^{+} \backslash J^{-}\right| \leqslant C N \xi$ и $\left|J \backslash J^{-}\right| \leqslant C N \xi$. Производя суммирование выражений из формулы (В.5) по $i \in J$ и используя соотношения (В.6) и (В.8), мы получаем

$$
\begin{aligned}
& \int_{E-b}^{E+b} \frac{\mathrm{d} E^{\prime}}{2 b} \int \sum_{i=1}^{N} Y_{i, \mathbf{m}}\left(E^{\prime}, \mathbf{x}\right) f_{\tau} \mathrm{d} \mu \\
& \quad \leqslant \frac{1}{2 b} \int \frac{1}{N} \sum_{i \in J} G\left(N\left(x_{i}-x_{i+m_{2}}\right), \ldots\right) f_{\tau} \mathrm{d} \mu+C b^{-1} \xi+C b^{-1} \xi^{-2} N^{-1-2 \mathfrak{a}}
\end{aligned}
$$


для каждого $\mathbf{m} \in S_{n}$. Аналогичная нижняя оценка может быть получена тем же способом. Положив $\xi=N^{-1 / 3}$, мы получаем

$$
\begin{aligned}
& \mid \int_{E-b}^{E+b} \frac{\mathrm{d} E^{\prime}}{2 b} \int \sum_{i=1}^{N} Y_{i, \mathbf{m}}\left(E^{\prime}, \mathbf{x}\right) f_{\tau} \mathrm{d} \mu \\
& \quad-\int \frac{1}{N} \sum_{i \in J} G\left(N\left(x_{i}-x_{i+m_{2}}\right), \ldots\right) f_{\tau} \mathrm{d} \mu \mid \leqslant C N^{-1 / 3}
\end{aligned}
$$

для каждого $\mathbf{m} \in S_{n}$, где $C$ зависит от $b$. Выбор величины $\xi$ можно оптимизировать в зависимости от $b$ и $\mathfrak{a}$, что приводит к эффективной оценке, упомянутой после теоремы 3.1. Однако в нашем изложении мы не будем стремиться к эффективной оценке; за подробностями мы отсылаем читателя к [6].

Суммируя неравенства (В.9) по всем $\mathbf{m} \in S_{n}(M)$, мы получаем

$$
\begin{aligned}
& \mid \int_{E-b}^{E+b} \frac{\mathrm{d} E^{\prime}}{2 b} \int \sum_{\mathbf{m} \in S_{n}(M)} \sum_{i=1}^{N} Y_{i, \mathbf{m}}\left(E^{\prime}, \mathbf{x}\right) f_{\tau} \mathrm{d} \mu \\
& \quad-\int \sum_{\mathbf{m} \in S_{n}(M)} \frac{1}{N} \sum_{i \in J} G\left(N\left(x_{i}-x_{i+m_{2}}\right), \ldots\right) f_{\tau} \mathrm{d} \mu \mid \leqslant C M^{n-1} N^{-1 / 3},
\end{aligned}
$$

и та же оценка имеет место для равновесного состояния, т. е. для значения $\tau=\infty$ в (В.10). Вычитая эти две формулы и применяя неравенство (3.30) из теоремы 3.3 к каждому слагаемому во втором члене (В.9), мы получаем

$$
\left|\int_{E-b}^{E+b} \frac{\mathrm{d} E^{\prime}}{2 b} \int \sum_{\mathbf{m} \in S_{n}(M)} \sum_{i=1}^{N} Y_{i, \mathbf{m}}\left(E^{\prime}, \mathbf{x}\right)\left(f_{\tau}-1\right) \mathrm{d} \mu\right| \leqslant C M^{n-1}\left(N^{-1 / 3}+N^{-\delta / 6}\right) .
$$

Полагая

$$
M:=N^{\min \{1 / 3, \delta / 6\} / n},
$$

мы получаем, что (В.11) обращается в нуль при $N \rightarrow \infty$, что доказывает равенство (В.3).

Шаг 2: случай большого m; доказательство равенства (В.4). Для данных $y \in \mathbb{R}, \ell>0$ пусть

$$
\chi(y, \ell):=\sum_{i=1}^{N} \mathbf{1}\left\{x_{i} \in\left[y-\frac{\ell}{N}, y+\frac{\ell}{N}\right]\right\}
$$

обозначает число точек в отрезке $[y-\ell / N, y+\ell / N]$. Заметим, что для данного $\mathbf{m}=\left(m_{2}, \ldots, m_{n}\right)$ мы имеем

$$
\sum_{i=1}^{N}\left|Y_{i, \mathbf{m}}\left(E^{\prime}, \mathbf{x}\right)\right| \leqslant C \chi\left(E^{\prime}, \ell\right) \mathbf{1}\left(\chi\left(E^{\prime}, \ell\right) \geqslant m_{n}\right) \leqslant C \sum_{m=m_{n}}^{\infty} m \mathbf{1}\left(\chi\left(E^{\prime}, \ell\right) \geqslant m\right)
$$

где $\ell$ обозначает максимум величины $\left|u_{1}\right|+\cdots+\left|u_{n}\right|$ по носителю функции $\widetilde{O}\left(u_{1}, \ldots, u_{n}\right)$. 
Сумма по всем возрастающим последовательностям $\mathbf{m}=\left(m_{2}, \ldots, m_{n}\right) \in$ $\mathbb{N}_{+}^{n-1}$ с фиксированным $m_{n}$ содержит не более $m_{n}^{n-2}$ членов, следовательно,

$$
\begin{aligned}
& \sum_{\mathbf{m} \in S_{n}^{c}(M)}\left|\int_{E-b}^{E+b} \frac{\mathrm{d} E^{\prime}}{2 b} \int \sum_{i=1}^{N}\right| Y_{i, \mathbf{m}}\left(E^{\prime}, \mathbf{x}\right)\left|f_{\tau} \mathrm{d} \mu\right| \\
& \leqslant C \int_{E-b}^{E+b} \frac{\mathrm{d} E^{\prime}}{2 b} \sum_{m=M}^{\infty} m^{n-1} \int \mathbf{1}\left(\chi\left(E^{\prime}, \ell\right) \geqslant m\right) f_{\tau} \mathrm{d} \mu .
\end{aligned}
$$

Теперь мы воспользуемся условием IV для отрезка $I=\left[E^{\prime}-N^{-1+\sigma}, E^{\prime}+N^{-1+\sigma}\right]$, где $\sigma:=(2 n)^{-1} \min \{1 / 3, \delta / 6\}$. Очевидно, что $\mathscr{N}_{I} \geqslant \chi\left(E^{\prime}, \ell\right)$ для достаточно большого $N$. Следовательно, из (3.10) мы получаем, что для любого $a \in \mathbb{N}$ выполнено неравенство

$$
\sum_{m=M}^{\infty} m^{n-1} \int \mathbf{1}\left(\chi\left(E^{\prime}, \ell\right) \geqslant m\right) f_{\tau} \mathrm{d} \mu \leqslant C_{a} \sum_{m=M}^{\infty} m^{n-1}\left(\frac{m}{N^{\sigma}}\right)^{-a} .
$$

В силу нашего выбора $\sigma$, неравенство $\sqrt{m} \geqslant N^{\sigma}$ выполнено для любого $m \geqslant M$ (см. (В.12)). Тогда, полагая $a=2 n+2$, мы получаем

$$
\sum_{m=M}^{\infty} m^{n-1} \int \mathbf{1}\left(\chi\left(E^{\prime}, \ell\right) \geqslant m\right) f_{\tau} \mathrm{d} \mu \leqslant \frac{C_{a}}{M} \rightarrow 0
$$

при $N \rightarrow \infty$. Подставляя это в (В.14), мы завершаем доказательство равенства (В.4). Тем самым теорема 3.1 полностью доказана.

\section{Список литературы}

[1] M. L. Mehta, Random matrices, Academic Press, Boston, MA, 1991, ISBN: 0-12-488051-7, xviii+562 pp.

[2] P. A. Deift, Orthogonal polynomials and random matrices: A Riemann-Hilbert approach, Courant Lect. Notes Math., 3, New York Univ., Courant Institute of Mathematical Sciences, New York; Amer. Math. Soc., Providence, RI, 1999, ISBN: 0-9658703-2-4; 0-8218-2695-6, viii+273 pp.

[3] G. W. Anderson, A. Guionnet, O. Zeitouni, An introduction to random matrices, Cambridge Stud. Adv. Math., 118, Cambridge Univ. Press, Cambridge, 2010, ISBN: 978-0-521-19452-5, xiv+492 pp.

[4] P. J. Forrester, Log-gases and random matrices, London Math. Soc. Monogr. Ser., 34, Princeton Univ. Press, Princeton, NJ, 2010, ISBN: 978-0-691-12829-0, 791 pp.

[5] A. Guionnet, Grandes matrices aléatoires et théorèmes d'universalité, Séminaire Bourbaki, 62ème année, 2009-2010, 33 pp.

[6] L. Erdös, B. Schlein, H.-T. Yau, J. Yin, "The local relaxation flow approach to universality of the local statistics for random matrices", Ann. Inst. Henri Poincaré (to appear); arXiv: 0911.3687.

[7] L. Erdos, H.-T. Yau, J. Yin, Rigidity of eigenvalues of generalized Wigner matrices, arXiv: 1007.4652 .

[8] L. Erdos, H.-T. Yau, J. Yin, Universality for generalized Wigner matrices with Bernoulli distribution, arXiv: 1003.3813. 
[9] L. Erdos, H.-T. Yau, J. Yin, Bulk universality for generalized Wigner matrices, arXiv: 1001.3453.

[10] F. J. Dyson, "Correlations between eigenvalues of a random matrix", Comm. Math. Phys., 19:3 (1970), 235-250.

[11] M.L. Mehta, "A note on correlations between eigenvalues of a random matrix", Comm. Math. Phys., 20:3 (1971), 245-250.

[12] E. Brézin, S. Hikami, "Correlations of nearby levels induced by a random potential", Nuclear Phys. B, 479:3 (1996), 697-706; "Spectral form factor in a random matrix theory", Phys. Rev. E (3), 55:4 (1997), 4067-4083.

[13] K. J. Johansson, "Universality of the local spacing distribution in certain ensembles of Hermitian Wigner matrices", Comm. Math. Phys., 215:3 (2001), 683-705.

[14] L. Erdős, S. Péché, J.A. Ramírez, B. Schlein, H.-T. Yau, "Bulk universality for Wigner matrices", Comm. Pure Appl. Math., 63:7 (2010), 895-925; arXiv: 0905.4176 .

[15] F. J. Dyson, "A Brownian-motion model for the eigenvalues of a random matrix", J. Math. Phys., 3:6 (1962), 1191-1198.

[16] L. Erdos, B. Schlein, H.-T. Yau, Universality of random matrices and local relaxation flow, arXiv: 0907.5605.

[17] T. Tao, V. Vu, "Random matrices: Universality of local eigenvalue statistics", Acta Math., 206:1 (2011), 127-204; arXiv: 0906.0510.

[18] L. Erdős, J. Ramírez, B. Schlein, T. Tao, V. Vu, H.-T. Yau, "Bulk universality for Wigner Hermitian matrices with subexponential decay", Math. Res. Lett., 17:4 (2010), 667-674, http://www.mathjournals.org/mrl/2010-017-004/ 2010-017-004-007.html.

[19] В. А. Марченко, Л. А. Пастур, "Распределение собственных значений в некоторых ансамблях случайных матриц”, Матем. сб., 72:4 (1967), 507-536; англ. пер.: V. A. Marčenko, L. A. Pastur, "Distribution of eigenvalues for some sets of random matrices", Math. USSR-Sb., 1:4 (1967), 457-483.

[20] K. W. Wachter, "Strong limits of random matrix spectra for sample covariance matrices of independent elements", Ann. Probab., 6:1 (1978), 1-18.

[21] E. P. Wigner, "Characteristic vectors of bordered matrices with infinite dimensions", Ann. of Math. (2), 62:3 (1955), 548-564.

[22] J. Wishart, "The generalized product moment distribution in samples from a normal multivariate population", Biometrika, 20A (1928), 32-52.

[23] J.H. Schenker, H. Schulz-Baldes, "Semicircle law and freeness for random matrices with symmetries or correlations", Math. Res. Lett., 12:4 (2005), 531-542, http://www.mathjournals.org/mrl/2005-012-004/2005-012-004-007.html.

[24] J. Schenker, H. Schulz-Baldes, "Gaussian fluctuations for random matrices with correlated entries", Int. Math. Res. Not. IMRN, 15 (2007), rnm047, 36 pp.

[25] P. W. Anderson, "Absences of diffusion in certain random lattices", Phys. Rev., 109:5 (1958), 1492-1505.

[26] M. V. Berry, M. Tabor, "Level clustering in the regular spectrum", Proc. R. Soc. Lond. Ser. A, 356:1686 (1977), 375-394.

[27] O. Bohigas, M. J. Giannoni, C. Schmit, "Characterization of chaotic quantum spectra and universality of level fluctuation laws", Phys. Rev. Lett., 52:1 (1984), 1-4.

[28] J. Fröhlich, T. Spencer, "Absence of diffusion in the Anderson tight binding model for large disorder or low energy", Comm. Math. Phys., 88:2 (1983), 151-184.

[29] J. Fröhlich, F. Martinelli, E. Scoppola, T. Spencer, "Constructive proof of localization in the Anderson tight binding model", Comm. Math. Phys., 101:1 (1985), $21-46$. 
[30] M. Aizenman, S. Molchanov, "Localization at large disorder and at extreme energies: an elementary derivation", Comm. Math. Phys., 157:2 (1993), 245-278.

[31] N. Minami, "Local fluctuation of the spectrum of a multidimensional Anderson tight binding model", Comm. Math. Phys., 177:3 (1996), 709-725.

[32] T. Spencer, Lifshitz tails and localization, Preprint, 1993.

[33] A. Elgart, "Lifshitz tails and localization in the three-dimensional Anderson model", Duke Math. J., 146:2 (2009), 331-360.

[34] A. Klein, "Absolutely continuous spectrum in the Anderson model on the Bethe lattice", Math. Res. Lett., 1:4 (1994), 399-407, http://www.mrlonline.org/mrl/ 1994-001-004/1994-001-004-001.html.

[35] M. Aizenman, R. Sims, S. Warzel, "Absolutely continuous spectra of quantum tree graphs with weak disorder", Comm. Math. Phys., 264:2 (2006), 371-389.

[36] R. Froese, D. Hasler, W. Spitzer, "Transfer matrices, hyperbolic geometry and absolutely continuous spectrum for some discrete Schrödinger operators on graphs", J. Funct. Anal., 230:1 (2006), 184-221.

[37] M. Aizenman, S. Warzel, "The canopy graph and level statistics for random operators on trees", Math. Phys. Anal. Geom., 9:4 (2007), 291-333.

[38] I. Rodnianski, W. Schlag, "Classical and quantum scattering for a class of long range random potentials", Int. Math. Res. Not., 2003:5 (2003), 243-300.

[39] J. Bourgain, "Random lattice Schrödinger operators with decaying potential: some higher dimensional phenomena", Geometric aspects of functional analysis, Lecture Notes in Math., 1807, Springer, Berlin, 2003, 70-98.

[40] S. A. Denisov, "Absolutely continuous spectrum of multidimensional Schrödinger operator", Int. Math. Res. Not., 2004:74, 3963-3982; arXiv: math/0408376.

[41] W. De Roeck, J. Fröhlich, "Diffusion of a massive quantum particle coupled to a quasi-free thermal medium", Comm. Math. Phys., 303:3 (2011), 613-707; arXiv: 0906.5178.

[42] T. Chen, "Localization lengths and Boltzmann limit for the Anderson model at small disorders in dimension 3", J. Stat. Phys., 120:1-2 (2005), 279-337.

[43] H. Spohn, "Derivation of the transport equation for electrons moving through random impurities", J. Statist. Phys., 17:6 (1977), 385-412.

[44] L. Erdös, H.-T. Yau, "Linear Boltzmann equation as the weak coupling limit of the random Schrödinger equation", Comm. Pure Appl. Math., 53:6 (2000), 667-735.

[45] L. Erdös, M. Salmhofer, H.-T. Yau, "Quantum diffusion for the Anderson model in the scaling limit", Ann. Henri Poincaré, 8:4 (2007), 621-685.

[46] K. B. Efetov, Supersymmetry in disorder and chaos, Cambridge Univ. Press, Cambridge, 1997, ISBN: 0-521-47097-8, xiv+441 pp.

[47] Y. V. Fyodorov, A. D. Mirlin, "Scaling properties of localization in random band matrices: A $\sigma$-model approach", Phys. Rev. Lett., 67:18 (1991), 2405-2409.

[48] J. Schenker, "Eigenvector localization for random band matrices with power law band width", Comm. Math. Phys., 290:3 (2009), 1065-1097.

[49] L. Erdős, A. Knowles, "Quantum diffusion and eigenfunction delocalization in a random band matrix model", Comm. Math. Phys., 303:2 (2011), 509-554; arXiv: 1002.1695 .

[50] L. Erdos, A. Knowles, Quantum diffusion and delocalization for band matrices with general distribution, arXiv: 1005.1838.

[51] M. Disertori, H. Pinson, T. Spencer, "Density of states for random band matrices", Comm. Math. Phys., 232:1 (2002), 83-124.

[52] M. Disertori, T. Spencer, "Anderson localization for a supersymmetric sigma model", Comm. Math. Phys., 300:3 (2010), 659-671; arXiv: 0910.3325. 
[53] M. Disertori, T. Spencer, M. R. Zirnbauer, "Quasi-diffusion in a 3D supersymmetric hyperbolic sigma model", Comm. Math. Phys., 300:2 (2010), 435-486; arXiv: 0901.1652 .

[54] T. Tao, V. Vu, M. Krishnapur, "Random matrices: universality of ESD's and the circular law", Ann. Probab., 38:5 (2010), 2023-2065; arXiv: 0807.4898.

[55] L. Erdős, B. Schlein, H.-T. Yau, "Semicircle law on short scales and delocalization of eigenvectors for Wigner random matrices", Ann. Probab., 37:3 (2009), 815-852.

[56] L. Erdős, B. Schlein, H.-T. Yau, "Local semicircle law and complete delocalization for Wigner random matrices", Comm. Math. Phys., 287:2 (2009), 641-655.

[57] L. Erdős, B. Schlein, H.-T. Yau, "Wegner estimate and level repulsion for Wigner random matrices", Int. Math. Res. Not. IMRN, 2010:3 (2010), 436-479; arXiv: 0811.2591.

[58] Z.D. Bai, Y. Q. Yin, "Limit of the smallest eigenvalue of a large dimensional sample covariance matrix", Ann. Probab., 21:3 (1993), 1275-1294.

[59] A. Guionnet, "Large deviations upper bounds and central limit theorems for non-commutative functionals of Gaussian large random matrices", Ann. Inst. H. Poincaré Probab. Statist., 38:3 (2002), 341-384.

[60] G. W. Anderson, O. Zeitouni, "A CLT for a band matrix model", Probab. Theory Related Fields, 134:2 (2006), 283-338.

[61] B. Simon, Trace ideals and their applications, 2nd ed., Math. Surveys Monogr., 120, Amer. Math. Soc., Providence, RI, 2005, ISBN: 0-8218-3581-5, viii+150 pp.

[62] A. Soshnikov, "Universality at the edge of the spectrum in Wigner random matrices", Comm. Math. Phys., 207:3 (1999), 697-733.

[63] Я.Г. Синай, А.Б. Сошников, "Уточнение полукругового закона Вигнера в окрестности края спектра для случайных симметричных матриц", Функи. анализ и его прил., 32:2 (1998), 56-79; англ. пер.: Ya. G. Sinai, A. B. Soshnikov, "A refinement of Wigner's semicircle law in a neighborhood of the spectrum edge for random symmetric matrices", Funct. Anal. Appl., 32:2 (1998), 114-131.

[64] T. Tao, V. Vu, "Random matrices: universality of local eigenvalue statistics up to the edge", Comm. Math. Phys., 298:2 (2010), 549-572; arXiv: 0908.1982.

[65] C. A. Tracy, H. Widom, "Level-spacing distributions and the Airy kernel", Comm. Math. Phys., 159:1 (1994), 151-174.

[66] C. A. Tracy, H. Widom, "On orthogonal and symplectic matrix ensembles", Comm. Math. Phys., 177:3 (1996), 727-754.

[67] A. Ruzmaikina, "Universality of the edge distribution of eigenvalues of Wigner random matrices with polynomially decaying distributions of entries", Comm. Math. Phys., 261:2 (2006), 277-296.

[68] S. Péché, A. Soshnikov, "Wigner random matrices with non-symmetrically distributed entries", J. Stat. Phys., 129:5-6 (2007), 857-884.

[69] T. Tao, V. Vu, "Random covariance matrices: Universality of local statistics of eigenvalues", Ann. Probab. (to appear); arXiv: 0912.0966.

[70] F. J. Dyson, "Statistical theory of energy levels of complex systems. I", J. Math. Phys., 3:1 (1962), 140-156; F. J. Dyson, "Statistical theory of energy levels of complex systems. II", J. Math. Phys., 3:1 (1962), 157-165; F. J. Dyson, "Statistical theory of energy levels of complex systems. III", J. Math. Phys., 3:1 (1962), 166-175.

[71] I. Dumitriu, A. Edelman, "Matrix models for beta ensembles", J. Math. Phys., 43:11 (2002), 5830-5847.

[72] J. Ramírez, B. Rider, B. Virag, Beta ensembles, stochastic Airy spectrum, and a diffusion, arXiv: math/0607331.

[73] J. A. Ramírez, B. Rider, "Diffusion at the random matrix hard edge", Comm. Math. Phys., 288:3 (2009), 887-906. 
[74] A. Edelman, B. D. Sutton, "From random matrices to stochastic operators", J. Stat. Phys., 127:6 (2007), 1121-1165.

[75] B. Valkó, B. Virág, "Continuum limits of random matrices and the Brownian carousel", Invent. Math., 177:3 (2009), 463-508.

[76] B. Valkó, B. Virág, "Large gaps between random eigenvalues", Ann. Probab., 38:3 (2010), 1263-1279; arXiv: 0811.0007.

[77] M. L. Mehta, M. Gaudin, "On the density of eigenvalues of a random matrix", $\mathrm{Nu}$ clear Phys., 18 (1960), 420-427.

[78] P. Bleher, A. Its, "Semiclassical asymptotics of orthogonal polynomials, Riemann-Hilbert problem, and universality in the matrix model", Ann. of Math. (2), 150:1 (1999), 185-266; arXiv: math-ph/9907025.

[79] P. Deift, T. Kriecherbauer, K. T.-R. McLaughlin, S. Venakides, X. Zhou, "Uniform asymptotics for polynomials orthogonal with respect to varying exponential weights and applications to universality questions in random matrix theory", Comm. Pure Appl. Math., 52:11 (1999), 1335-1425.

[80] P. Deift, T. Kriecherbauer, K. T.-R. McLaughlin, S. Venakides, X. Zhou, "Strong asymptotics of orthogonal polynomials with respect to exponential weights", Comm. Pure Appl. Math., 52:12 (1999), 1491-1552.

[81] A. S. Fokas, A. R. Its, A. V. Kitaev, "The isomonodromy approach to matric models in 2D quantum gravity", Comm. Math. Phys., 147:2 (1992), 395-430.

[82] L. Pastur, M. Shcherbina, "Bulk universality and related properties of Hermitian matrix models", J. Stat. Phys., 130:2 (2008), 205-250.

[83] D. S. Lubinsky, "A new approach to universality limits involving orthogonal polynomials", Ann. of Math. (2), 170:2 (2009), 915-939.

[84] E. Levin, D.S. Lubinsky, "Universality limits in the bulk for varying measures", Adv. Math., 219:3 (2008), 743-779.

[85] G. Ben Arous, S. Péché, "Universality of local eigenvalue statistics for some sample covariance matrices", Comm. Pure Appl. Math., 58:10 (2005), 1316-1357.

[86] K. Johansson, Universality for certain Hermitian Wigner matrices under weak moment conditions, arXiv: 0910.4467.

[87] C. Itzykson, J.-B. Zuber, "The planar approximation. II", J. Math. Phys., 21:3 (1980), 411-421.

[88] E. Brézin, S. Hikami, "An extension of the Harish-Chandra-Itzykson-Zuber integral", Comm. Math. Phys., 235:1 (2003), 125-137.

[89] L. Erdős, J. Ramírez, B. Schlein, H.-T. Yau, "Universality of sine-kernel for Wigner matrices with a small Gaussian perturbation", Electron. J. Probab., 15 (2010), 526-603, Paper 18, http://www.math.washington.edu/ ${ }^{\sim}$ ejpecp/viewarticle.php?id= 2084\&layout=abstract; arXiv: 0905.2089.

[90] M. Shcherbina, "Edge universality for orthogonal ensembles of random matrices", J. Stat. Phys., 136:1 (2009), 35-50; arXiv: 0812.3228.

[91] D. Bakry, M. Émery, "Diffusions hypercontractives", Séminaire de probabilités XIX, Lecture Notes in Math., 1123, Springer, Berlin, 1985, 177-206.

[92] J. Gustavsson, "Gaussian fluctuations of eigenvalues in the GUE", Ann. Inst. H. Poincaré Probab. Statist., 41:2 (2005), 151-178.

[93] S. O'Rourke, "Gaussian fluctuations of eigenvalues in Wigner random matrices", J. Stat. Phys., 138:6 (2010), 1045-1066.

[94] R. Curto, L. Fialkow, "Recursiveness, positivity and truncated moment problems", Houston J. Math., 17:4 (1991), 603-635.

[95] O. N. Feldheim, S. Sodin, "A universality result for the smallest eigenvalues of certain sample covariance matrices", Geom. Funct. Anal., 20:1 (2010), 88-123; arXiv: 0812.1961 . 
[96] S. Sodin, "The spectral edge of some random band matrices", Ann. of Math. (2), 172:3 (2010), 2223-2251; arXiv: 0906.4047.

[97] S. Sodin, "The Tracy-Widom law for some sparse random matrices", J. Stat. Phys., 136:5 (2010), 834-841; arXiv: 0903.4295.

[98] S. Péché, A. Soshnikov, "On the lower bound of the spectral norm of symmetric random matrices with independent entries", Electron. Commun. Probab., 13 (2008), 280-290, http://www.math.washington.edu/ ejpecp/ECP/viewarticle.php? $\mathrm{id}=2004 \&$ layout $=$ abstract.

[99] A. Auffinger, G. Ben Arous, S. Péché, "Poisson convergence for the largest eigenvalues of heavy tailed random matrices", Ann. Inst. Henri Poincaré Probab. Statist., 45:3 (2009), 589-610.

[100] G. Biroli, J.-P. Bouchaud, M. Potters, "On the top eigenvalue of heavy-tailed random matrices", Europhys. Lett. EPL, 78:1 (2007), Art. 10001, 5 pp.

[101] D. L. Hanson, F. T. Wright, "A bound on tail probabilities for quadratic forms in independent random variables", Ann. Math. Statist., 42:3 (1971), 1079-1083.

[102] E. B. Davies, "The functional calculus", J. London Math. Soc. (2), 52:1 (1995), 166-176.

[103] T. Spencer, "Random banded and sparse matrices (Chapter 23)", Oxford handbook of random matrix theory, eds. G. Akemann, J. Baik, P. Di Francesco, Oxford Univ. Press, Oxford, 2011, ISBN: 978-0-19-957400-1, 960 pp.

[104] Z. D. Bai, B. Miao, J. Tsay, "Convergence rates of the spectral distributions of large Wigner matrices", Int. Math. J., 1:1 (2002), 65-90.

[105] T. Tao, V. Vu, Random matrices: localization of the eigenvalues and the necessity of four moments, arXiv: 1005.2901.

[106] H.-T. Yau, "Relative entropy and the hydrodynamics of Ginzburg-Landau models", Lett. Math. Phys., 22:1 (1991), 63-80.

[107] D. W. Stroock, Probability theory: an analytic view, Cambridge Univ. Press, Cambridge, 1993, ISBN: 0-521-43123-9, xvi+512 pp.

[108] А.Н. Ширяев, Вероятность, 3-е изд., МЦНМО, М., 2007; 1-е изд., Наука, М., 1980; англ. пер. 1-го изд.: А. N. Shiryayev, Probability, Grad. Texts in Math., 95, Springer-Verlag, New York, 1984, xi+577 pp.

Л. Эрдёш (L. Erdös)

Ludwig-Maximilians-Universität München

E-mail: lerdos@mathematik. uni-muenchen.de
Поступила в редакцию

07.04.2010 